\title{
International Union of Basic and Clinical Pharmacology. CV. Somatostatin Receptors: Structure, Function, Ligands, and New Nomenclature
}

Thomas Günther, Giovanni Tulipano, Pascal Dournaud, Corinne Bousquet, Zsolt Csaba, Hans-Jürgen Kreienkamp, Amelie Lupp, Márta Korbonits, Justo P. Castaño, Hans-Jürgen Wester, Michael Culler, ${ }^{1}$ Shlomo Melmed, and Stefan Schulz

Institute of Pharmacology and Toxicology, Jena University Hospital, Friedrich-Schiller-University, Jena, Germany (T.G., A.L., S.S.); Unit of Pharmacology, Department of Molecular and Translational Medicine, University of Brescia, Brescia, Italy (G.T.); PROTECT, INSERM, Université Paris Diderot, Sorbonne Paris Cité, Paris, France (P.D., Z.C.); Cancer Research Center of Toulouse, INSERM UMR 1037-University Toulouse III Paul Sabatier, Toulouse, France (C.B.); Institute of Human Genetics, University Medical Center Hamburg-Eppendorf, Hamburg, Germany (H.-J.K.); Centre for Endocrinology, William Harvey Research Institute, Barts and London School of Medicine, Queen Mary University of London, London, United Kingdom (M.K.); Maimonides Institute for Biomedical Research of Cordoba, Córdoba, Spain (J.P.C.); Department of Cell Biology, Physiology, and Immunology, University of Córdoba, Córdoba, Spain (J.P. C.); Reina Sofia University Hospital, Córdoba, Spain (J.P.C.); CIBER Fisiopatología de la Obesidad y Nutrición, Córdoba, Spain (J.P.C.); Pharmaceutical Radiochemistry, Technische Universität München, Munich, Germany (H.-J.W.); Culler Consulting LLC, Hopkinton, Massachusetts (M.C.); and Pituitary Center, Department of Medicine, Cedars-Sinai Medical Center, Los Angeles, California (S.M.)

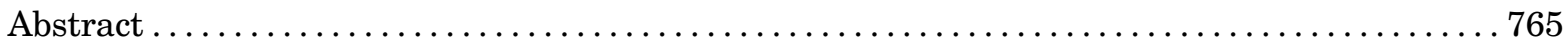

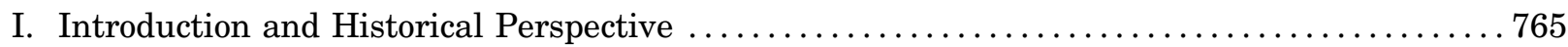

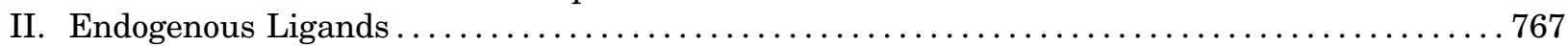

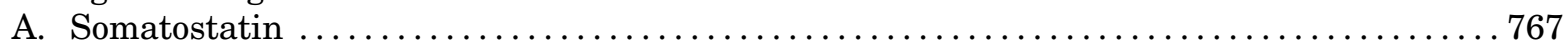

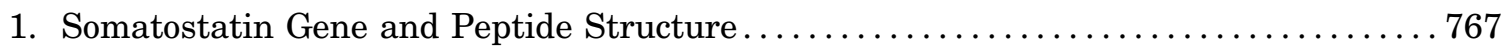

2. Regulation of Gene Expression and Peptide Release $\ldots \ldots \ldots \ldots \ldots \ldots \ldots \ldots \ldots \ldots$

3. Anatomic Framework ............................................ 768

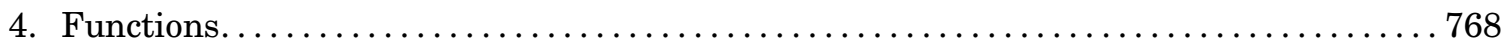

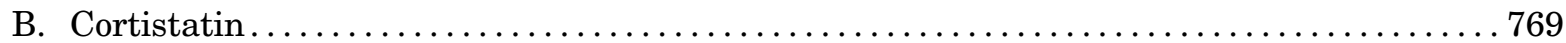

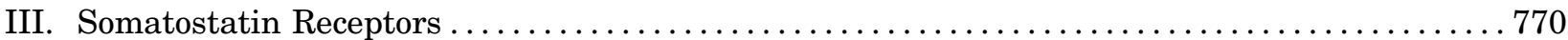

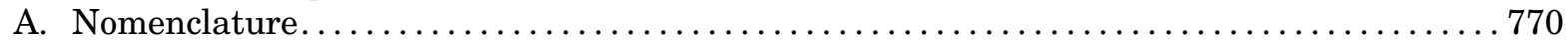

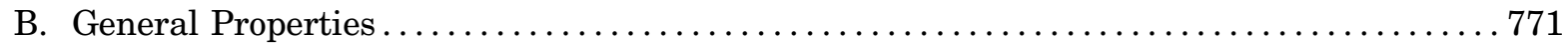

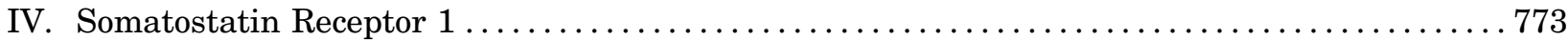

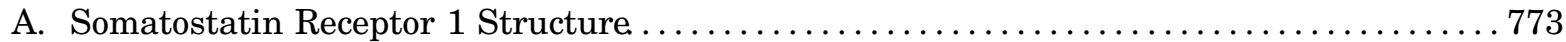

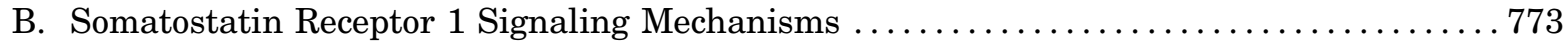

C. Somatostatin Receptor 1 Regulation and Trafficking $\ldots \ldots \ldots \ldots \ldots \ldots \ldots \ldots \ldots \ldots \ldots \ldots \ldots$

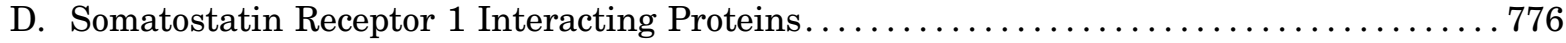

E. Somatostatin Receptor 1 Anatomic Framework ......................... 776

Address correspondence to: Dr. Stefan Schulz, Institute of Pharmacology and Toxicology, Jena University Hospital - Friedrich Schiller University Jena, Drackendorfer Straße 1, D-07747 Jena, Germany. E-mail: Stefan.Schulz@med.uni-jena.de

This work was supported by the Deutsche Forschungsgemeinschaft [Grant GRK 1459 to H.-J.K.; Grants SCHU924/10-3, SCHU924/14-1, and SFB/TR166-TPC5 to S.S.]; the Deutsche Krebshilfe [Grant 109952 to S.S.]; the Inserm and Paris Diderot University to P.D. and Z.C.; the French National Institute for Cancer Research [INCa Grants 2013-102 and 2015-115 to C.B.]; the French Ligue Nationale Contre le Cancer [Grant Equipe Labellisée to C.B.]; the Université Fédérale de Toulouse [Grant U1037-G16001BB-IDEX to C.B.]; the University of Brescia [Grants 2015 and 2016_DMMT_Tulipano_EX60 to G.T.]; the Junta de Andalucia [Grants BIO-0139 and CTS-1406 to J.P.C.]; the MINECO [Grant BFU2016-80360-R to J.P.C.]; the ISCIII [Grant PIE14-00005, CIBERobn to J.P.C.], the GETNE [Grant 2014, SAEDYN Grant 2016 to J.P.C.]; the Medical Research Council and EU FP7 to M.K.; the National Institutes of Health [Grant DK103198 to S.M.]; and the Doris Factor Molecular Endocrinology Laboratory at Cedars-Sinai Medical Center. G.T. has received research grants (2009 and 2015$)$ from Novartis Farma (Italy). C.B. has received research grants (2012-2017) from Novartis. M.K. has received research grants and lecture fees from Pfizer and Ipsen, and is a consultant for Ono, Heptares, Ferring, and Pfizer. J.P.C. has received research grants and lecture fees from Novartis and Ipsen, and is listed as inventor of patent PCT/ES2007/00627. M.C. was previously employed by Ipsen. S.M. has received research grants from Pfizer and Ipsen, and is a consultant for Chiasma, Novartis, Ionis, Ono, Strongbridge, and Midatech. S.S. has received research grants and lecture fees (2006-2017) from Novartis, Ipsen, Aspireo, Italfarmaco, and Strongbridge.

T.G., P.D., Z.C., H.-J.K., A.L., and H.-J.W. have nothing to disclose.

https://doi.org/10.1124/pr.117.015388. 


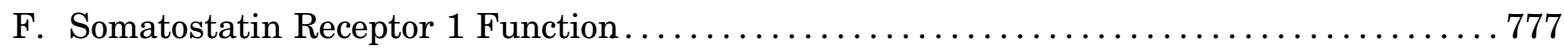

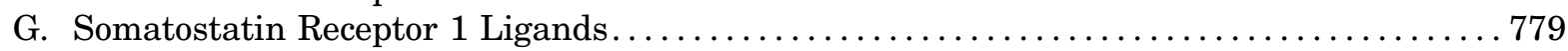

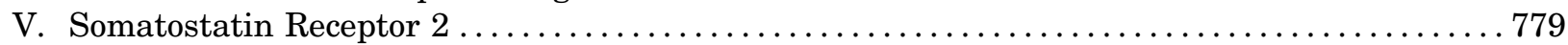

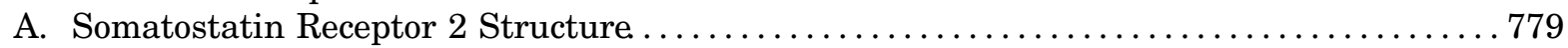

B. Somatostatin Receptor 2 Signaling Mechanisms $\ldots \ldots \ldots \ldots \ldots \ldots \ldots \ldots \ldots \ldots \ldots \ldots 780$

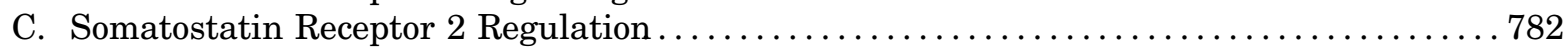

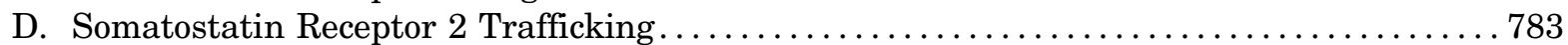

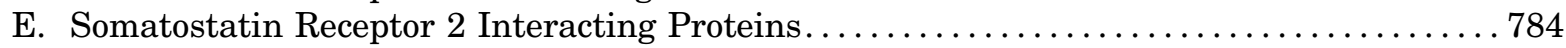

F. Somatostatin Receptor 2 Anatomic Framework $\ldots \ldots \ldots \ldots \ldots \ldots \ldots \ldots \ldots \ldots \ldots \ldots \ldots \ldots$

1. Central and Peripheral Nervous System.............................. 785

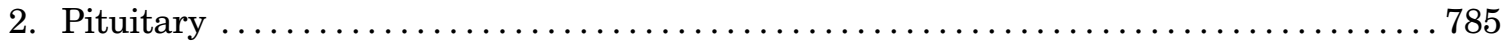

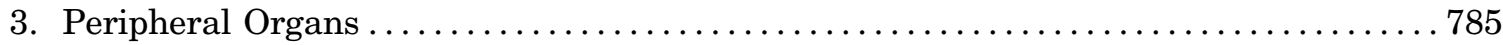

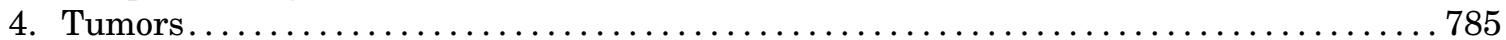

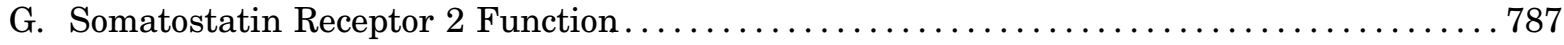

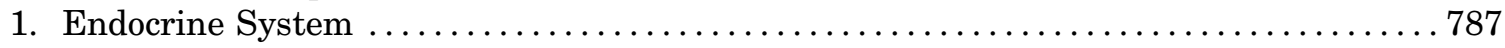

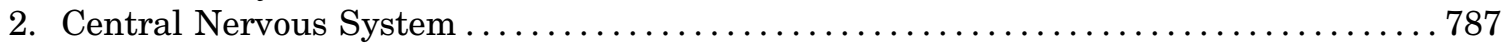

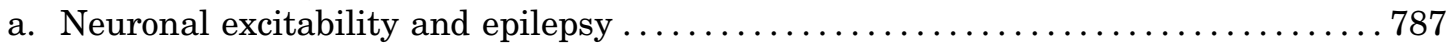

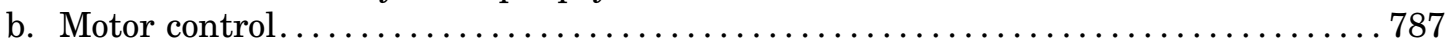

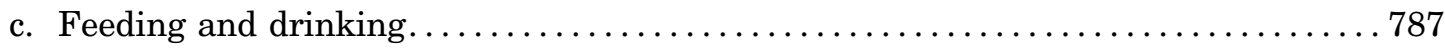

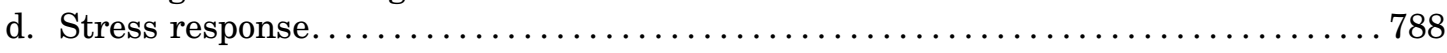

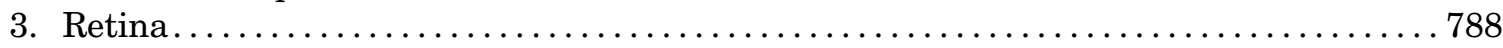

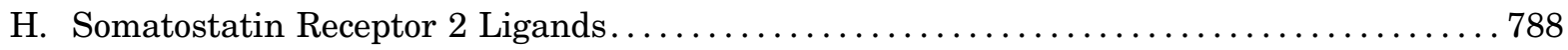

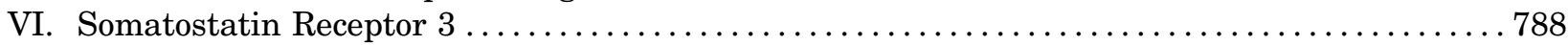

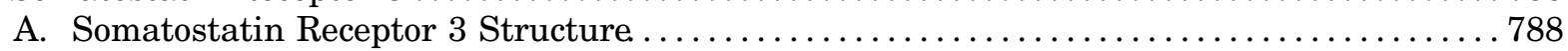

B. Somatostatin Receptor 3 Signaling Mechanisms $\ldots \ldots \ldots \ldots \ldots \ldots \ldots \ldots \ldots \ldots \ldots \ldots$

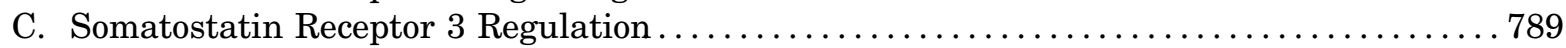

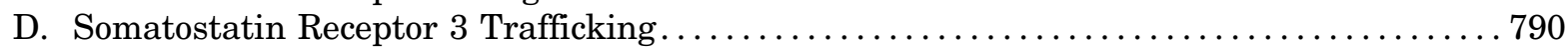

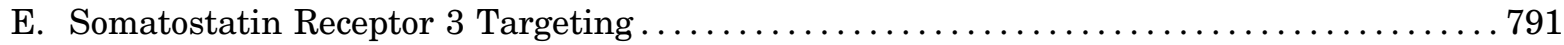

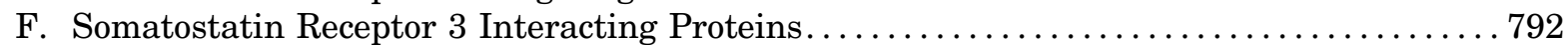

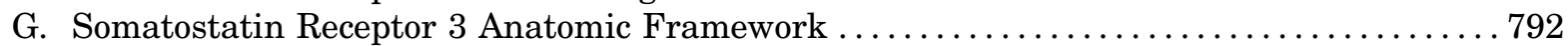

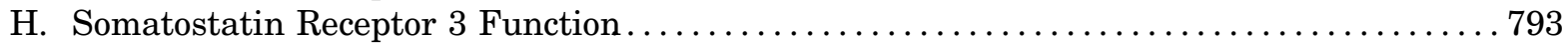

I. Somatostatin Receptor 3 Ligands....................................... 793

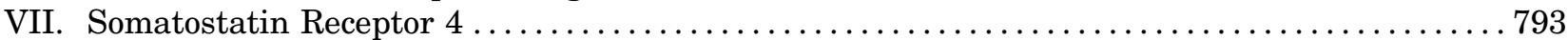

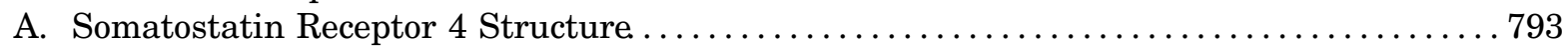

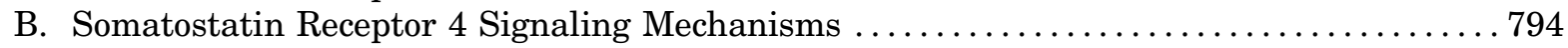

C. Somatostatin Receptor 4 Regulation ................................ 794

\begin{abstract}
ABBREVIATIONS: ACTH, adrenocorticotropic hormone; AIP, aryl hydrocarbon receptor-interacting protein; AP, activator protein; ARC, arcuate nucleus; BBS, Bardet-Biedl syndrome; CA, Cornu Ammonis; CCK, cholecystokinin; CD26, dipeptidyl peptidase-4/cluster of differentiation 26; CHO, Chinese hamster ovary; CNS, central nervous system; CRF, corticotropin-releasing factor; CRH, corticotropinreleasing hormone; CST, cortistatin; $\mathrm{D}_{2}$ receptor, dopamine receptor D2; DOTA, 1,4,7,10-tetraazacyclododecane-1,4,7,10-tetraacetic acid; DOTANOC, DOTA-D-Nal ${ }^{3}$-octreotide; DOTATATE, DOTA-Tyr ${ }^{3}$-octreotate; DOTATOC, DOTA-D-Tyr ${ }^{3}$-octreotide; DRG, dorsal root ganglion; DTPA, diethylenetriaminepentaacetic acid; ECE-1, endothelin-converting enzyme 1; ECL, extracellular loop; ERK, extracellular signalregulated kinase; FDA, Food and Drug Administration; FGF, fibroblast growth factor; FLNA, filamin A; GEP, gastroenteropancreatic; GH, growth hormone; GHRH, GH-releasing hormone; GHS-R1a, ghrelin receptor 1a; GI, gastrointestinal; GIST, GI stromal tumor; GIT, GI tract; GPCR, G protein-coupled receptor; GRK, G protein-coupled receptor kinase; HCC, hepatocellular carcinoma; HEK, human embryonic kidney; ICL, intracellular loop; IGF-1, insulin-like growth factor 1; IL, interleukin; ITIM, immunoreceptor tyrosine-based inhibition motif; IUPHAR, International Union of Basic and Clinical Pharmacology; JAK2, Janus kinase 2; KO, knockout; LAR, long-acting release; LNPEP, leucylcysteinyl aminopeptidase; mAb, monoclonal antibody; MAPK, mitogen-activated protein kinase; MIBP1, c-myc intron binding protein 1; mTOR, mammalian target of rapamycin; MUPP1, multi PDZ-domain protein 1; NET, neuroendocrine tumor; NHE1, sodium/hydrogen exchanger 1; NHERF, sodium/hydrogen exchanger regulatory factor 1; NODAGA, 1,4,7-triazacyclononane,1-glutaric acid-4,7-acetic acid; NOS, nitric oxide synthase; PDZ, PSD-95/discs large/ZO-1; PET, positron emission tomography; PI3K, phosphatidylinositol-4,5-bisphosphate 3-kinase; PIST, protein interacting specifically with Tc10; PKA, protein kinase A; PLC, phosphoinositide-specific phospholipase C; PNGase F, peptide N-glycosidase F; PP1, protein phosphatase 1; PRI, peptide receptor imaging; PRRT, peptide-receptor radiotherapy; PSD, postsynaptic density; PTP, protein tyrosine phosphatase; $\mathrm{PTP}_{\eta}$, protein tyrosine phosphatase $\eta$; PTX, pertussis toxin; Rab, Ras-related in brain; Ras, rat sarcoma; Rho, Ras homolog; SEF-2, helix-loop-helix transcription factor; SHP, Src homology region 2 domain-containing phosphatase; SPECT, single-photon emission computed tomography; SRIF, somatotropin-release inhibitory factor; SST, somatostatin receptor; $\mathrm{SST}_{5} \mathrm{TMD} \mathrm{SST}_{5}$ transmembrane domain; $t_{1 / 2}$, half-life; T3, triiodthyronine; TATE, Tyr ${ }^{3}$-octreotate; TGN, trans-Golgi network; TMD, transmembrane domain; TSH, thyroid-stimulating hormone; TSHoma, TSH-secreting pituitary adenoma; UFC, urinary-free cortisol; UTR, untranslated region; VOCC, voltage-operated calcium channel; Zac1, zinc finger protein 1.
\end{abstract}




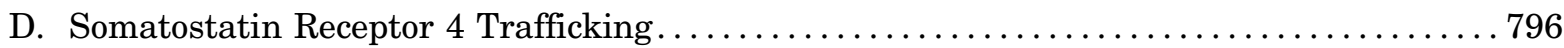

E. Somatostatin Receptor 4 Interacting Proteins.............................. 796

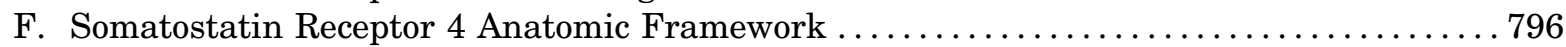

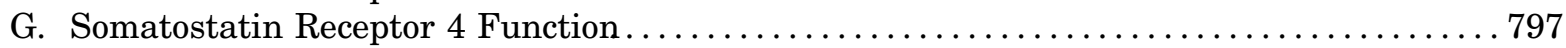

H. Somatostatin Receptor 4 Ligands..................................... 797

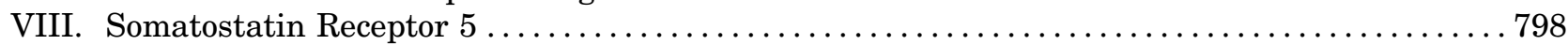

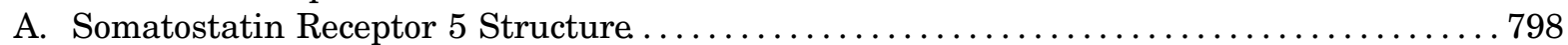

B. Somatostatin Receptor 5 Signaling Mechanisms $\ldots \ldots \ldots \ldots \ldots \ldots \ldots \ldots \ldots \ldots \ldots \ldots \ldots \ldots$

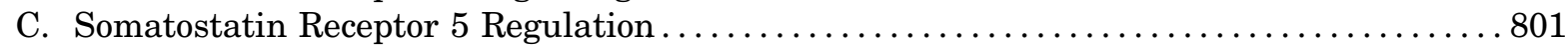

1. Regulation of Somatostatin Receptor 5 Gene Expression ................... 801

2. Ligand-Dependent Regulation of Somatostatin Receptor $5 \ldots \ldots \ldots \ldots \ldots \ldots \ldots \ldots$. $\ldots$.

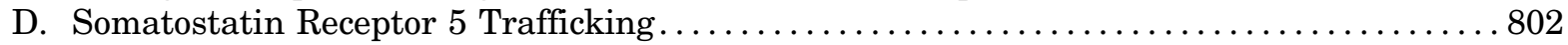

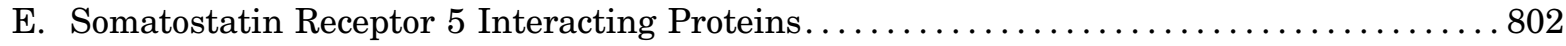

F. Somatostatin Receptor 5 Anatomic Framework . . . . . . . . . . . . . . . . . . . . . . . 804

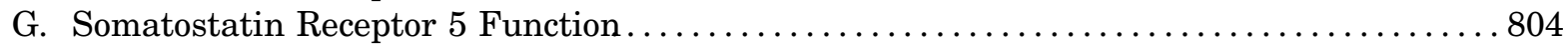

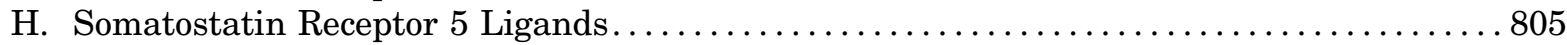

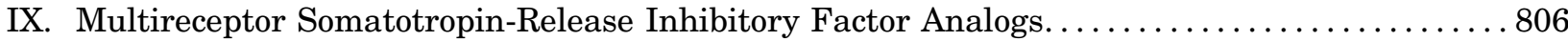

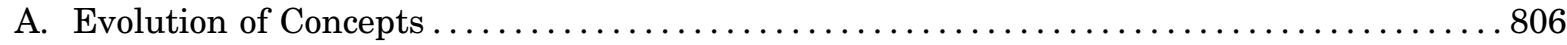

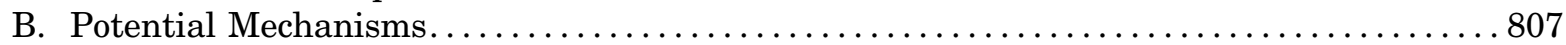

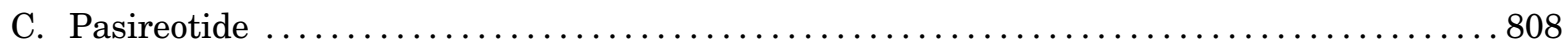

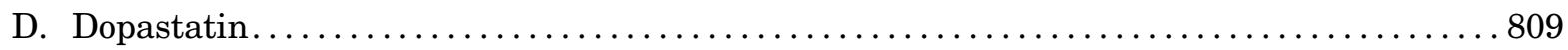

X. Somatotropin-Release Inhibitory Factor Analogs in Current Clinical Practice............. 810

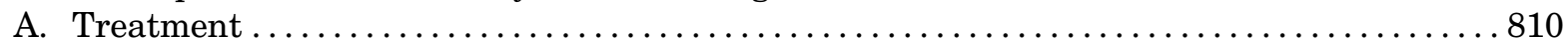

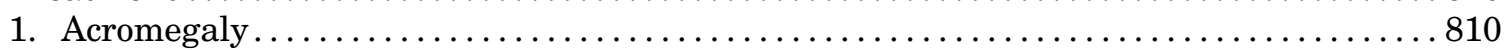

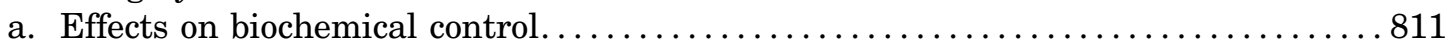

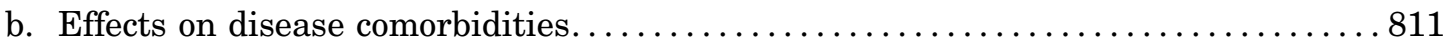

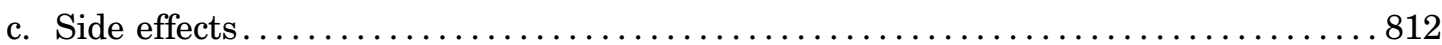

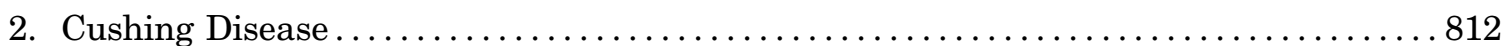

3. Thyroid-Stimulating Hormone-Secreting Pituitary Adenomas . . . . . . . . . . . . . 812

4. Neuroendocrine Tumors ...................................... 812

B. Factors Influencing Somatotropin-Release Inhibitory Factor Analog Resistance ........ 812

C. Somatotropin-Release Inhibitory Factor-Based Radiopharmaceuticals . . . . . . . . . 814

1. Radiolabeled Agonists .......................................... 814

2. Pan Somatotropin-Release Inhibitory Factor-Like Peptides $\ldots \ldots \ldots \ldots \ldots \ldots \ldots \ldots . \ldots 17$

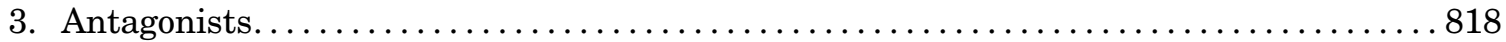

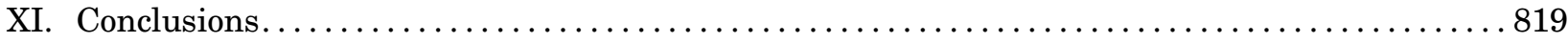

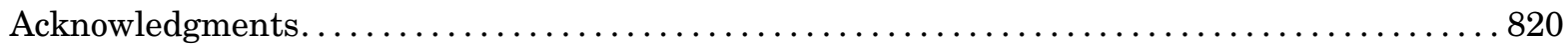

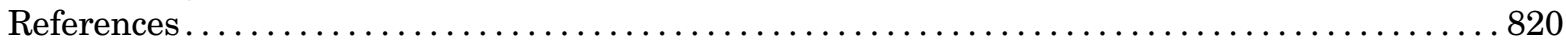

\begin{abstract}
Somatostatin, also known as somatotropinrelease inhibitory factor, is a cyclopeptide that exerts potent inhibitory actions on hormone secretion and neuronal excitability. Its physiologic functions are mediated by five $G$ protein-coupled receptors (GPCRs) called somatostatin receptor (SST)1-5. These five receptors share common structural features and signaling mechanisms but differ in their cellular and subcellular localization and mode of regulation. $\mathrm{SST}_{2}$ and $\mathrm{SST}_{5}$ receptors
\end{abstract}

have evolved as primary targets for pharmacological treatment of pituitary adenomas and neuroendocrine tumors. In addition, $\mathbf{S S T}_{2}$ is a prototypical GPCR for the development of peptide-based radiopharmaceuticals for diagnostic and therapeutic interventions. This review article summarizes findings published in the last 25 years on the physiology, pharmacology, and clinical applications related to SSTs. We also discuss potential future developments and propose a new nomenclature.

\section{Introduction and Historical Perspective}

Since their discovery, research on somatostatin and its receptors has remained active with more than 700 papers published annually. Somatostatin-also known as somatotropin release-inhibiting factor (SRIF) - was originally discovered in 1973 as a hypothalamic neuropeptide based on its ability to inhibit growth hormone (GH) release from the anterior pituitary (Fig. 1) (Brazeau et al., 1973). SRIF occurs in two forms, SRIF-14 and SRIF-28, with broad antisecretory activity on many hormones, including GH, insulin, glucagon, gastrin, cholecystokinin (CCK), and ghrelin. In the original report, it was suggested that SRIF could have potential for treatment of acromegaly. However, due to its short circulating half-life $\left(t_{1 / 2}\right)$ 


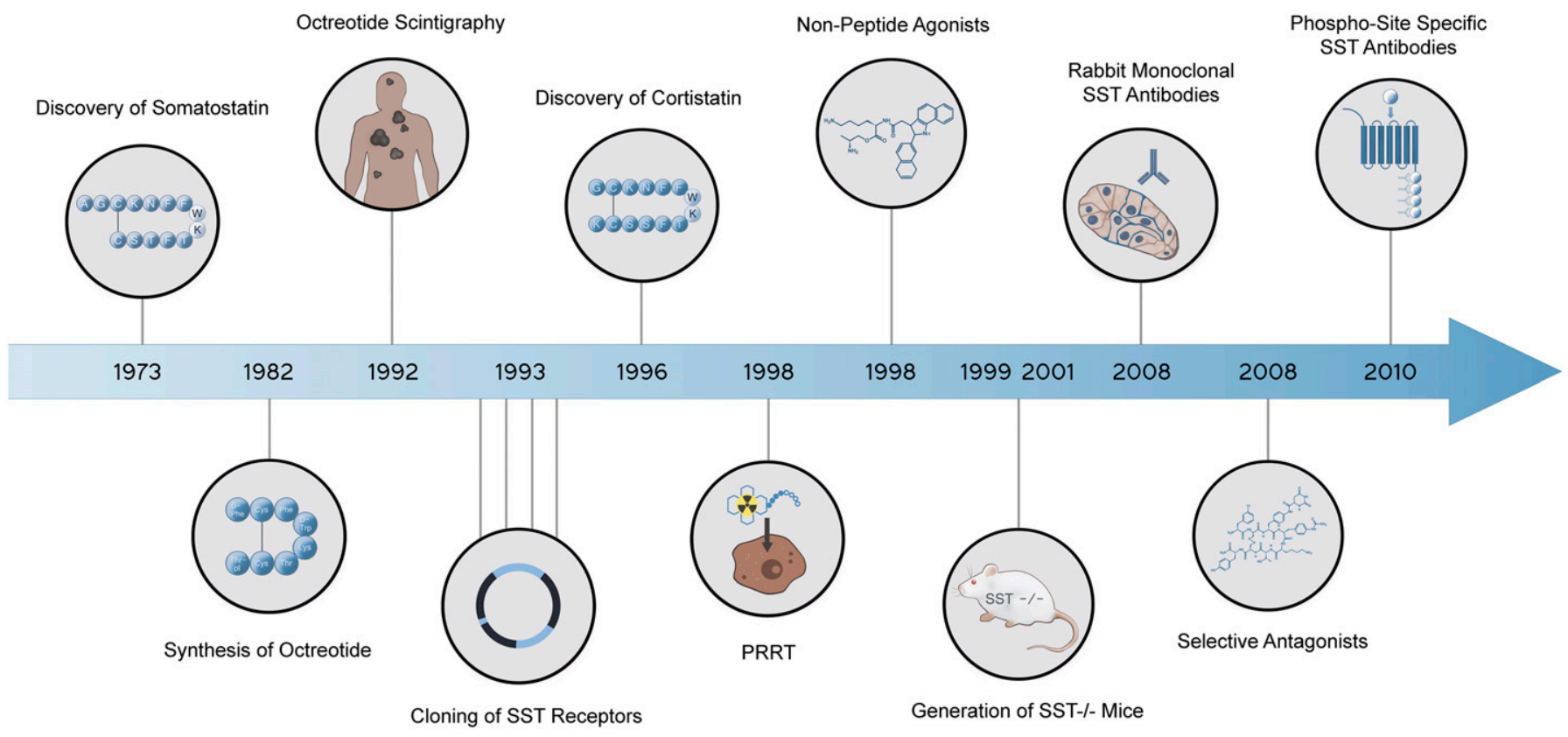

Fig. 1. Historical perspective of somatostatin and somatostatin receptor research.

( $<3$ minutes), the therapeutic potential of natural SRIF-14 is limited. Consequently, highly potent and metabolically stable SRIF analogs were synthesized in 1982 (Bauer et al., 1982). The first SRIF analog approved for clinical use was octreotide (Lamberts et al., 1996). About 10 years later, the first SRIF-based radiopharmaceuticals were synthesized by conjugating a chelator to octreotide, followed by radiolabeling with a $\gamma$-emitter, which paved the way for in vivo imaging of human tumors (Bakker et al., 1991a,b; Krenning et al., 1993). In the early 1990s, five subtypes of somatostatin receptors (SSTs) termed $\mathrm{SST}_{1}$ to $\mathrm{SST}_{5}$ were cloned in mice, rats, and humans (Meyerhof et al., 1991, 1992; Bruno et al., 1992; Kluxen et al., 1992; Li et al., 1992; O'Carroll et al., 1992; Yamada et al., 1992a,b, 1993; Yasuda et al., 1992; Rohrer et al., 1993; Panetta et al., 1994; Schwabe et al., 1996; Lublin et al., 1997). Given that only two SRIF tissue binding sites could be identified using ligands available at that time, the subsequent discovery of five different SSTs was surprising and triggered in-depth research into binding properties, localization, and regulation of the ligand. This led to classification of the clinically used SRIF analogs octreotide and lanreotide as $\mathrm{SST}_{2}$-prefering ligands, which in turn stimulated the search for novel compounds that bind either more broadly or more selectively to individual SSTs. In 1996, a structurally related neuropeptide termed cortistatin (CST) with a more restricted distribution in the cerebral cortex and hippocampus was identified (de Lecea et al., 1996). In the late 1990s, knockout (KO) mice and selective nonpeptide agonists were developed for all five SSTs, which helped to define their physiologic functions (Zheng et al., 1997; Kreienkamp et al., 1999; Strowski et al., 2003; Tirone et al., 2003; Qiu et al., 2005, 2008; Tallent et al., 2005; Einstein et al., 2010). In 1998, the development of octreotide conjugates radiolabeled with a $\beta$-emitter provided proof-of-principle for peptide-receptor radiotherapy (PRRT) (Stolz et al., 1998). A few years later, the capacity of SSTs to form homodimers and heterodimers with other G protein-coupled receptors (GPCRs) was observed, which stimulated the search for bitopic chimeric compounds (Rocheville et al., 2000a; Pfeiffer et al., 2001, 2002). In the early 2000s, the search for multireceptor ligands led to the discovery of pasireotide, which was the first pituitary-directed drug approved for therapy of Cushing's disease (Bruns et al., 2002; Colao et al., 2012). In 2008, high-affinity peptide antagonists with utility for SST imaging and treatment were synthesized, suggesting that receptor internalization is not an absolute requirement for tumor imaging (Cescato et al., 2008). Also in 2008, the generation of highly specific rabbit monoclonal antibodies (mAbs) facilitated detection of SSTs in human tissues and enabled correlation of $\mathrm{SST}_{2}$ and $\mathrm{SST}_{5}$ receptor expression with octreotide and pasireotide responses (Fischer et al., 2008; Lupp et al., 2011). Shortly thereafter, development of phosphosite-specific antibodies provided molecular insights into mechanisms for SST activation by octreotide and pasireotide (Poll et al., 2010; Petrich et al., 2013; Lehmann et al., 2016). In 2009 , a novel truncated variant of $\mathrm{SST}_{5}$ generated by aberrant splicing was identified $\mathrm{SST}_{5}$ transmembrane domain ( $\mathrm{SST}_{5} \mathrm{TMD}$ )] and shown to be overexpressed in several hormone-related tumors, wherein the variant increases aggressiveness (Durán-Prado et al., 2009, 2012b; Gahete et al., 2010a; HormaecheaAgulla et al., 2017). In the past few years, orally available and subtype-selective SST agonists and antagonists have been synthesized. Some of these 
substances may become lead compounds for potential new therapeutic indications directed toward individual SSTs (He et al., 2014; Hirose et al., 2017).

\section{Endogenous Ligands}

\section{A. Somatostatin}

1. Somatostatin Gene and Peptide Structure. SRIF, a cyclic neuroendocrine peptide, was first isolated and identified as a hypothalamic factor that inhibited GH secretion from anterior pituitary cells (Brazeau et al., 1973). SRIF exists in two main bioactive isoforms: the tetradecapeptide (SRIF-14) isolated from the hypothalamus and the 28-amino-acid isoform (SRIF-28), generated from the same prepro-SRIF precursor through post-translational processing at a distinct cleavage site and which differs from the shorter isoform by an N-terminal extension (Fig. 2) (Esch et al., 1980; Pradayrol et al., 1980; Shen and Rutter, 1984). Both isoforms are expressed at variable amounts in the same tissue areas. It is not clear whether the two peptides are coexpressed by the same cells or by separate cells. The family of somatostatin peptides includes CST, a highly similar peptide reviewed below, and which is structurally and functionally related to the urotensin II peptide family. The two families (somatostatin and urotensin II) as well as those of their respective GPCRs may derive from a single ancestral ligand-receptor pair. The duplication, generating the two families, likely occurred before the emergence of vertebrates. Subsequently, each family expanded during evolution, through whole-genome duplications, followed by local duplications and gene losses (Tostivint et al., 2014). Despite their evolutional divergence, the two families conserve close functional links (Malagon et al., 2008). The vertebrate SRIF family is composed of at least six paralogous genes named $S S 1$ to $S S 6$ (Liu et al., 2010). In mammals, SRIF-14 and SRIF-28 both derive from the SS1 gene, localized on chromosome $3 \mathrm{q} 27.3$ in humans. The SRIF-14 primary structure is highly conserved in vertebrates, and cleavage sites generating SRIF-14 and its extended isoform have been fully conserved during evolution (Conlon et al., 1997). An additional product of the mammalian processing of prepro-SRIF is a 13-amino-acid noncyclic amidated peptide, neuronostatin, which immediately follows the signal peptide (Samson et al., 2008; Yosten et al., 2015). Bioinformatic analyses of evolutionary conserved sequences suggest the occurrence of neuronostatin in other vertebrates. A novel peptide showing structural similarity to SRIF-28 and isolated from monkey ileum comprises amino acid sequences matching the N-terminal 13 amino acids of SRIF-28. This peptide
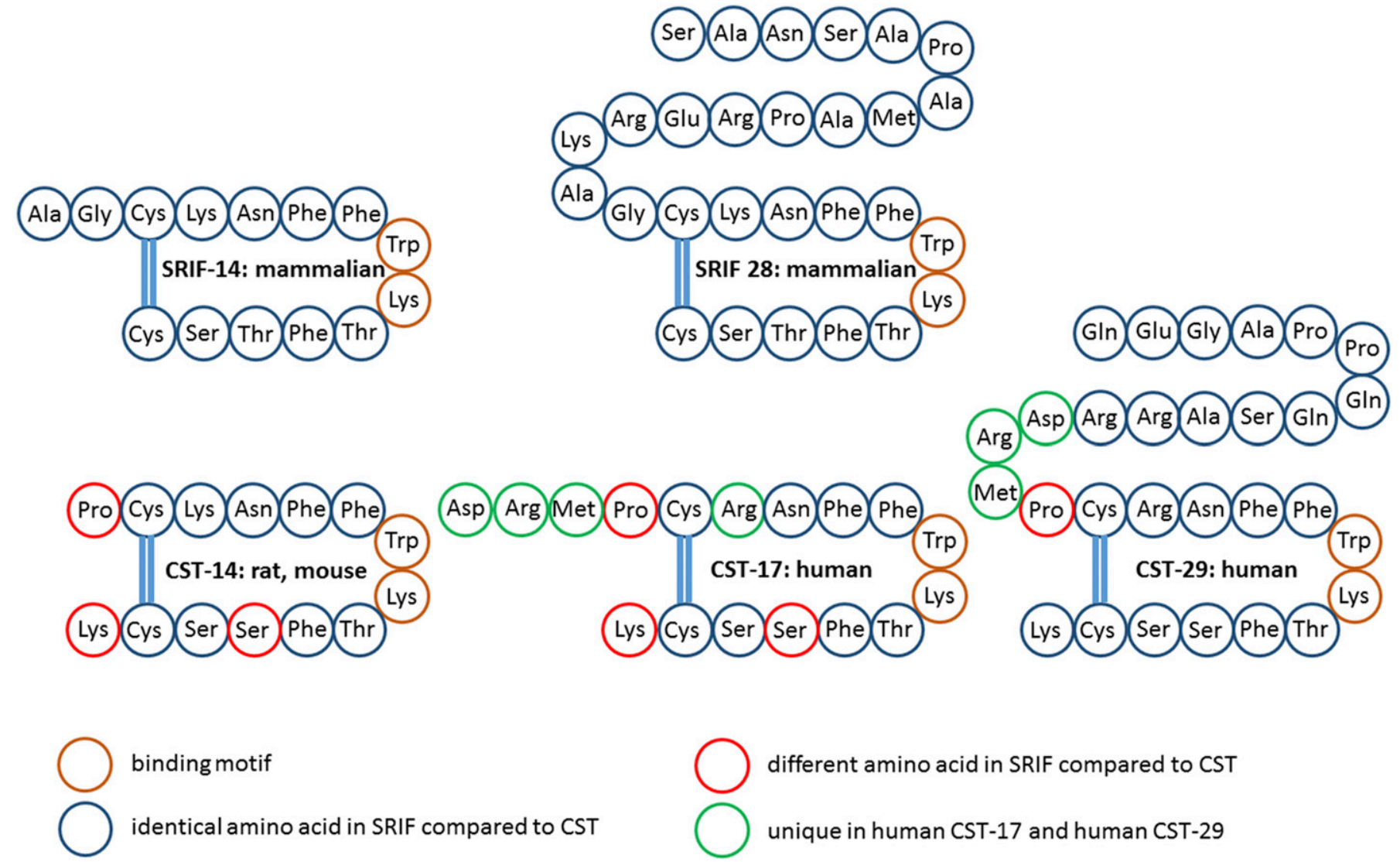

binding motif

identical amino acid in SRIF compared to CST

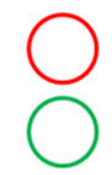

different amino acid in SRIF compared to CST

unique in human CST-17 and human CST-29

Fig. 2. Primary and secondary amino acid structure of mammalian SRIF and CST isoforms. Color code: brown, binding motif; blue, identical in SRIF and CST; red, different in CST compared with SRIF; green, not present in rat/mouse CST-14. 
is expressed in enteric neurons and may play a possible role in food intake control (Ensinck et al., 2002, 2003).

2. Regulation of Gene Expression and Peptide Release. The structure of rat and human SRIF genes, as well as the transcriptional unit and upstream regulatory elements of the rat gene, has been characterized (Montminy et al., 1984; Shen and Rutter, 1984). Expression of the SRIF precursor gene is regulated by growth factors and cytokines, including GH, insulin-like growth factor 1 (IGF-1), insulin, leptin, and inflammatory cytokines, and by glucocorticoids, testosterone, and estradiol. cAMP is a potent activator of SRIF transcription, and SRIF release from neurons and peripheral secretory cells is triggered by membrane depolarization and increased cytosolic calcium concentrations. Several neurotransmitters, neuropeptides, hormones, and nutrients, some also altering gene transcription, affect SRIF release in the central nervous system (CNS) and in peripheral tissues (Montminy et al., 1996; Müller et al., 1999; Patel, 1999; Eigler and Ben-Shlomo, 2014). Characterization of neurotransmitter, neuropeptide, and hormone modulation of hypothalamic SRIFergic neurons has raised interest in light of the key role played by SRIF as a distal mediator for neuroendocrine and metabolic control of the GH axis activity in health and disease (Müller et al., 1999).

3. Anatomic Framework. Abundant SRIF immunoreactivity is evident in the mediobasal hypothalamus and median eminence, amygdala, preoptic area, hippocampus, striatum, cerebral cortex, olfactory regions, and the brainstem (Johansson et al., 1984). Three main categories of SRIFergic neurons can be distinguished: hypophysiotropic neurons, long-projecting GABAergic neurons, and GABAergic interneurons acting within microcircuits (Viollet et al., 2008; Urban-Ciecko and Barth, 2016).

In the rat CNS, SRIFergic neurons regulating pituitary function are located within the periventricular nucleus and the parvocellular part of the paraventricular nucleus and send axonal projections to the median eminence at the base of the hypothalamus. SRIFproducing neuronal cell bodies are also found in the arcuate (ARC) and ventromedial nuclei. Hypophysiotropic SRIFergic neuronal axons descend toward the pituitary stalk and release SRIF into the portal blood vessel system, thereby reaching anterior pituitary cells. Some axons travel through the neural pituitary stalk into the neurohypophysis. Other fibers project outside the hypothalamus to areas such as the limbic system or may interact, through interneurons, with other hypothalamic nuclei, including the ARC where GH-releasing hormone (GHRH) is expressed, the preoptic nucleus, the ventromedial nucleus, and the suprachiasmatic nucleus, which exhibits circadian pacemaker activity (Müller et al., 1999; Eigler and Ben-Shlomo, 2014). SRIF is ubiquitously expressed in mammalian brain. Extrahypothalamic SRIF immunoreactivity is found in the amygdala, preoptic area, hippocampus, striatum, cerebral cortex, sensory regions, and brainstem. SRIF neurons are classified into two main categories: interneurons acting locally within microcircuits and longrange-projecting neurons whose fibers reach distant areas. SRIF frequently colocalizes with GABA. SRIFergic interneurons likely play a role in regulation of distal dendrite excitability, and long-range-projecting SRIFergic neurons may participate in coordinating activity between distant brain regions. Accordingly, nonpyramidal cells located in the hippocampus and targeting the medial septum and the medial entorhinal cortex form inhibitory synapses on GABAergic interneurons, and may cooperate in generation and synchronization of rhythmic oscillatory activity in these areas (Viollet et al., 2008; Melzer et al., 2012). Recently, brain-wide SRIF neuron maps have established the precise cell number, density, and somatic morphology with anatomic references for SRIF-related specific functions (Kim et al., 2017; Zhang et al., 2017).

Peripheral SRIF is produced by secretory cells in gastrointestinal mucosa and by $\delta$-cells in pancreatic islets, as well as by a subpopulation of $C$ cells within the thyroid gland. In addition to SRIF-producing neuroendocrine cells, inflammatory and immune response cells and tumor cells may also express SRIF (Patel, 1999). The peptide has been immunolocalized in human epidermis, with low expression levels in keratinocytes and higher expression in subsets of Merkel and dendritic cells (Vockel et al., 2010, 2011). SRIF is a neurotransmitter and neuromodulator, an endocrine hormone and a paracrine factor acting in the same tissue where it is expressed. Circulating levels of SRIF are very low because the peptide is rapidly degraded by ubiquitous peptidases (Rai et al., 2015). In the retina, SRIF was detected by immunohistochemical studies in amacrine and ganglion cells, as well as in interplexiform cells, whereas electrophysiological studies support the view that it may function as a neurotransmitter, neuromodulator, or trophic factor (Cervia et al., 2008).

4. Functions. Within the hypothalamus-pituitary system, SRIF is the main regulatory element exerting inhibitory control on both basal and stimulated GH secretion and reduces prolactin and thyroid-stimulating hormone (TSH) secretion in normal subjects (Müller et al., 1999). It can also suppress release of adrenocorticotropic hormone (ACTH) from tumor cells (Hofland et al., 2010). SRIF brain actions are mediated by presynaptic or postsynaptic mechanisms. SRIF modulates neuronal excitability, and in the hippocampus, cortex, and hypothalamus it also induces presynaptic inhibition of excitatory neurotransmission (Peineau et al., 2003). In other brain areas, SRIF also decreases GABA release. Postsynaptic mechanisms of action include membrane hyperpolarization via activation of potassium ion currents ( $\mathrm{K}^{+}$currents), in particular voltage-gated $\mathrm{K}^{+}$currents, noninactivating potassium currents ( $\mathrm{M}$ currents), and voltage-insensitive leak 
currents (Moore et al., 1988; Schweitzer et al., 1998; Jiang et al., 2003; Qiu et al., 2008). SRIF is coreleased with GABA from hippocampal neurons and from axonal terminals in other brain areas (Olias et al., 2004). SRIF inhibits dopamine release from the midbrain as well as hypothalamic release of noradrenaline, thyrotropinreleasing hormone, and corticotropin-releasing hormone (CRH) (Patel, 1999). Activation of brain SRIF signaling may alleviate endocrine, autonomic, and behavioral responses to stress mediated by central CRH and CRH receptors (Stengel and Taché, 2017). SRIF has a role in cognitive functions, learning and memory processes, control of locomotor activity, control of food intake, nociception, and autonomic functions. SRIF is highly expressed in brain regions associated with seizures and has been suggested as an endogenous antiepileptic (Olias et al., 2004; Tallent and Qiu, 2008; Stengel et al., 2015).

Peripheral SRIF actions include inhibition of hormone secretion, exocrine secretion, and cell proliferation. In the gastrointestinal (GI) tract (GIT), SRIF exerts a generalized inhibitory effect on release of gut hormones [including gastrin, CCK, gastric inhibitory polypeptide, vasoactive intestinal peptide, enteroglucagon, motilin], gastric acid, digestive enzymes, bile, and colonic fluid. SRIF also negatively affects gallbladder contraction, small intestinal segmentation, and gastric emptying. In pancreatic islets, release of SRIF from $\delta$-cells inhibits secretion of insulin, glucagon, and other peptides from neighboring cells. SRIF reduces TSHinduced release of triiodothyronine (T3) and thyroxine as well as calcitonin release. In the adrenals, SRIF inhibits angiotensin II-stimulated aldosterone secretion and acetylcholine-stimulated medullary catecholamine secretion. SRIF reduces release of kidney-derived renin caused by hypovolemia and vasopressin-mediated water absorption. In addition to nervous system functions and regulation of endocrine and GI functions, SRIF also may affect key cellular processes in diverse tissues by regulating the release of both growth factors and cytokines as well as cellular responses to these stimuli. SRIF can contribute to control of smooth muscle cell contractility, lymphocyte and inflammatory cell proliferation and activity, tumor cell growth, and normal tissue plasticity (Patel, 1999; Rai et al., 2015). In human skin, SRIF has been suggested as a negative regulator of epidermal wound healing (Vockel et al., 2011). Finally, at variance with its nearly universal inhibitory actions, low (pM) concentrations of SRIF stimulate in vitro GH release on cultured pituitary cells derived from pigs (Luque et al., 2006) and nonhuman primates (Cordoba-Chacon et al., 2012b) and from human somatotroph adenomas (Matrone et al., 2004).

\section{B. Cortistatin}

CST, a cyclic neuropeptide, highly homologous to SRIF, was identified as a region-specific brain mRNA encoding a protein of 112-amino-acid residues, which was called preproCST (de Lecea et al., 1996). CST in mammals derives from the CORT gene (Liu et al., 2010). The gene encoding for human and mouse CST is located on 1p36.3-1p36.2 and on chromosome 4, respectively. Similarly to preproSRIF, cleavage of preproCST gives rise to multiple mature products, CST-14 and CST-29 in rats and CST-17 and CST-29 in humans (Fig. 2). CST-14 and SRIF-14 differ in three amino acid; CST aligns with the second-amino-acid residue of SRIF on the $\mathrm{N}$-terminus and terminates one-amino-acid residue beyond the C-terminal of SRIF (de Lecea et al., 1997b). Human CST-17 contains an arginine for lysine substitution and is extended by three amino acids at the amino-terminal end, resulting in CST-17 sharing 10 of the 14 SRIF-14 residues. Similar to their prepropeptides, mature CST and SRIF are also highly homologous, including the two cysteine residues that render the peptides cyclic as well as a FWKT motif critical for SST binding (de Lecea et al., 1997b). Consequently, CST peptides bind to all SST subtypes with similar affinity than SRIF, and yet there is no evidence for a selective cortistatin receptor (Siehler et al., 2008). Notably, the FWKT motif is also present in urotensin II and urotensin-related peptide, which are indeed agonists of SSTs (Vaudry et al., 2015).

PreproCST mRNA is predominantly expressed in the cerebral cortex and hippocampus. In the cortex, mainly layers II-III and VI contain CST-positive cells. Interestingly, CST-positive cell bodies are not uniformly distributed in all cortical areas, with highest numbers evident in the visual and temporal cortex and lowest in the somatosensory cortex (de Lecea et al., 1997a). CSTcontaining neurons are also detected in the piriform cortex and entorhinal area. In the hippocampus, CST expression is found in a small subset of nonpyramidal neurons of the subiculum and in the stratum oriens of hippocampus subfields Cornu Ammonis (CA)1-3. In the hilar region of the dentate gyrus, however, CST-positive neurons are only transiently present during development (de Lecea et al., 1997a). In parallel, there is a temporary increase of cortical CST expression during development, which correlates with maturation of cortical interneurons. Indeed, cortical CST-expressing neurons, similarly to SRIF-positive neurons, are also GABAergic. However, CST- and SRIF-containing neurons are expressed in distinct, only partially overlapping populations (de Lecea et al., 1997a). CST is also expressed in other brain areas, such as in the olfactory bulb, in the striatum, in the periventricular nucleus of the hypothalamus, and in GABAergic interneurons of the deep layers of the spinal cord dorsal horn (de Lecea, 2008; Morell et al., 2013). No CST expression was detected in the thalamus, brainstem, or cerebellum (de Lecea, 2008). The projections of CST-positive neurons were not analyzed in detail, but due to their high homology it is possible that anti-SRIF antibodies may also label CST-containing axons. 
CST is also expressed in the periphery, in general at lower level than SRIF but with a broader distribution: preproCST mRNA was detected in peripheral nociceptive neurons, endocrine organs (e.g., pituitary gland, adrenal gland, thyroid gland, parathyroid gland, endocrine pancreas), digestive system (e.g., stomach, jejunum, ileum, colon, rectum, liver), kidney, lung, and gonads, and also in smooth muscle cells, endothelial cells, and immune cells (e.g., lymphocytes, monocytes, macrophages, dendritic cells) (Broglio et al., 2007; Gahete et al., 2008; Gonzalez-Rey et al., 2015). Discrepancies between mRNA expression and protein synthesis were observed in several tissues (including adrenal, thyroid, lung, and gonads) (Broglio et al., 2007). Notably, predominant CST is present in parathyroid chief cells and immune cells (Dalm et al., 2003a; Allia et al., 2005).

In keeping with their similar affinities to SSTs, CST and SRIF share several biologic properties, including inhibition of neuronal activity and consequent antiepileptic activity (Braun et al., 1998; Aourz et al., 2014), inhibition of cell proliferation, and regulation of hormones, and particularly inhibition of $\mathrm{GH}$ secretion (Spier and de Lecea, 2000). Double KO mice devoid of both SRIF and CST show markedly increased GH levels, although they do not display overt giant phenotypes (Pedraza-Arevalo et al., 2015). Nevertheless, functional differences of CST and SRIF can only partly be attributed to their distinct tissue distributions (de Lecea and Castaño, 2006). At the cellular level, CST, similarly to SRIF, increases the M current in hippocampal neurons but also augments the hyperpolarizationactivated currents (Schweitzer et al., 2003), thereby modulating synaptic integration and regulation of oscillatory activity. At the behavioral level, CST induces hypomotility, whereas SRIF causes hypermotility (Criado et al., 1999); CST enhances slow-wave sleep, whereas SRIF increases rapid eye movement sleep (de Lecea et al., 1996; Bourgin et al., 2007). CST and SRIF also regulate differently endocrine functions (IbáñezCosta et al., 2017b) as well as learning and memory processes (Borbély et al., 2013). Consistent with its widespread distribution in the immune system (Dalm et al., 2003b), CST is a potent anti-inflammatory factor, decreasing the production of several inflammatory cytokines [tumor necrosis factor- $\alpha$, interleukin (IL)- $1 \beta$, IL-6, IL-12, interferon- $\gamma$ ], chemokines, and acute-phase proteins (Gonzalez-Rey et al., 2015). CST also inhibits T helper 1- and 17-driven inflammatory responses in models of inflammation [e.g., sepsis (Gonzalez-Rey et al., 2006a), atherosclerosis (Delgado-Maroto et al., 2017)], and autoimmune diseases [e.g., inflammatory bowel disease (GonzalezRey et al., 2006b), rheumatoid arthritis (Gonzalez-Rey et al., 2007), and multiple sclerosis (Souza-Moreira et al., 2013)]. In parallel with its potent anti-inflammatory effect, CST is also an endogenous analgesic factor acting at both the peripheral and spinal level (Morell et al., 2013).
CST may activate GPCRs other than SSTs (IbáñezCosta et al., 2017b), including the ghrelin receptor 1a (GHS-R1a) (Callaghan and Furness, 2014) and humanspecific MAS-related GPR family member X2 (Solinski et al., 2014). Functions of CST not shared by SRIF in the immune (Gonzalez-Rey et al., 2015) and endocrine (Cordoba-Chacon et al., 2011) systems are likely mediated by GHS-R1a, whereas MAS-related GPR family member X2 might play an important role in the analgesic effects of CST in humans. In addition, some CST-specific functions might be mediated by a yet unidentified CST-selective receptor (Gonzalez-Rey et al., 2015), and by truncated $\mathrm{SST}_{5}$ variants that selectively respond to CST (Gahete et al., 2008; Durán-Prado et al., 2009; Cordoba-Chacon et al., 2010, 2011; Ibáñez-Costa et al., 2017b).

\section{Somatostatin Receptors}

\section{A. Nomenclature}

There is yet considerable misconception and lack of clarity regarding classification and nomenclature of SSTs. SRIF binding sites were initially defined through radioligand-binding studies performed in rat brain cerebral cortex membranes. SRIF-1 (also called SS-1) recognition sites were characterized by high affinity for SRIF-14 and SRIF-28, and for cyclic peptides such as octreotide and seglitide. In contrast, SRIF-2 (also called SS-2) sites exhibit high affinity for SRIF-14 and SRIF28 , but very low affinity for octreotide and seglitide. In fact, SS-1 and SS-2 binding sites correlate with recombinant $\mathrm{SST}_{2}$ and $\mathrm{SST}_{1}$ receptors, respectively (Hoyer et al., 1995b; Schoeffter et al., 1995).

Subsequently, five distinct receptor genes have been cloned and named chronologically according to their respective publication dates, but two were regrettably given the same appellation $\left(\mathrm{SST}_{4}\right)$. In 1995, a consistent nomenclature for the recombinant receptors was agreed upon according to International Union of Basic and Clinical Pharmacology (IUPHAR) guidelines ( $\mathrm{sst}_{1}, \mathrm{sst}_{2}$, sst $_{3}$, sst $_{4}$, and sst $_{5}$ ) (Hoyer et al., 1995a). Given that radioligands could differentiate only two distinct SRIF binding sites, the subsequent cloning of five receptors was indeed surprising. IUPHAR guidelines recommended that recombinant receptors without well-defined functional characteristics should be referred to by lower case letters, i.e., sst $_{1}$, sst ${ }_{2}$, etc. (Vanhoutte et al., 1996). When the recombinant receptor is shown to be of functional relevance in whole tissues and is fully characterized, upper case letters should be used, i.e., $\mathrm{SST}_{1}, \mathrm{SST}_{2}$, etc. (Vanhoutte et al., 1996). Moreover, the name of a receptor should not include the letter "R" or " $r$ " as an abbreviation for receptor (Vanhoutte et al., 1996). Thus, according to IUPHAR guidelines, employing receptor names such as SSTR1, SSTR2, etc., is discouraged (Vanhoutte et al., 1996).

Shortly after cloning, it became apparent that the five recombinant receptors comprise two classes or groups, 
on the basis of their phylogeny, structural homologies, and pharmacological properties. One class was referred to as SRIF1, comprising $\mathrm{SST}_{2}, \mathrm{SST}_{3}$, and $\mathrm{SST}_{5}$ receptor subtypes. The other class was referred to as SRIF2, comprising the other two recombinant receptor subtypes $\mathrm{SST}_{1}$ and $\mathrm{SST}_{4}$ (Hoyer et al., 1995a). SST subtypes share many structural characteristics and their main intracellular signaling pathways. Conversely, individual SST subtypes can now clearly be differentiated according to their cellular and subcellular localization as well as distinct modes of regulation and functional and pharmacological properties. The IUPHAR Committee on Receptor Nomenclature and Drug Classification subcommittee now recognizes the physiologic correlates of SSTs and has decided on upper case nomenclature for all five SSTs. Thus, the new recommended nomenclature for $\mathrm{SST}$ is $\mathrm{SST}_{1}, \mathrm{SST}_{2}, \mathrm{SST}_{3}$, $\mathrm{SST}_{4}$, and $\mathrm{SST}_{5}$ (Alexander et al., 2017; Schulz et al., 2017).

\section{B. General Properties}

All five SSTs are prototypical class A GPCRs that belong to the rhodopsin-like family of receptors. All possess seven transmembrane domains (TMDs) that provide the characteristic architecture of GPCRs. Receptor sequences for human SSTs range in length from 364 amino acids for $\mathrm{SST}_{5}$ to 418 amino acids for $\mathrm{SST}_{3}$ (Table 1). Unfortunately, crystal structures are not yet available for any SST. However, the five SST subtypes share common structural features such as a conserved sequence (YANSCANPILY) in transmembrane region 7 (mammalian SST signature). In addition, there is a consensus motif $(\mathrm{X}-[\mathrm{S} / \mathrm{T}]-\mathrm{X}-\Phi)$ at the end of the carboxylterminal tail of all mammalian SSTs. The X-S/T-X- $\Phi$ motif is regarded as a potential postsynaptic density protein (PSD)-95/discs large/ZO-1 (PDZ) domain binding site crucial for interaction with scaffolding proteins. Like all prototypical GPCRs, SSTs contain a DRY motif in the second intracellular loop (ICL) and are involved in coupling to $\mathrm{G}$ proteins. Genes encoding human $\mathrm{SST}_{1}-$ $\mathrm{SST}_{5}$ are located on chromosomes $14,17,22,20$, and 16, respectively (Table 1 ). There is considerable sequence similarity between different SST subtypes (39\%-57\%) (Table 2). In fact, sequence similarity is high for a given subtype when compared across species (81\%-98\% for mouse, human, and rat homologs).

Comparative genomic analysis suggests that the current set of receptors present in mammalian species arose from a single ancestral gene. This precursor was duplicated before the appearance of vertebrates, leading to genes coding for ancestral SRIF1-type and SRIF2type receptors, and one gene coding for the ligand, SRIF (Ocampo Daza et al., 2012; Tostivint et al., 2014). Further tetraploidizations occurred during vertebrate evolution, generating genes coding for $\mathrm{SST}_{2}, \mathrm{SST}_{3}$, and $\mathrm{SST}_{5}$ from the SRIF1-type precursor gene, and genes coding for $\mathrm{SST}_{1}, \mathrm{SST}_{4}$, and $\mathrm{SST}_{6}$ from the SRIF2-type precursor gene. The gene coding for $\mathrm{SST}_{6}$ has been lost

TABLE 1

Nomenclature and properties of somatostatin receptors

\begin{tabular}{|c|c|c|c|c|c|}
\hline & $\mathrm{SST}_{1}$ & $\mathrm{SST}_{2}$ & $\mathrm{SST}_{3}$ & $\mathrm{SST}_{4}$ & $\mathrm{SST}_{5}$ \\
\hline Gennomic location & $14 q 13$ & $17 q 24$ & $22 q 13.1$ & $20 \mathrm{p} 11.2$ & $16 \mathrm{p} 13.3$ \\
\hline Amino acids & 391 & 369 & 418 & 388 & 364 \\
\hline \multirow{2}{*}{$\begin{array}{l}\text { Naturally occurring } \\
\text { agonists }\end{array}$} & SRIF-14, SRIF-28 & SRIF-14, SRIF-28 & SRIF-14, SRIF-28 & SRIF-14, SRIF-28 & SRIF-14, SRIF-28 \\
\hline & CST-17, CST-29 & CST-17, CST-29 & CST-17, CST-29 & CST-17, CST-29 & CST-17, CST-29 \\
\hline G protein coupling & $\mathrm{G} \alpha_{\mathrm{i} / \mathrm{o}}$ & $\mathrm{G} \alpha_{\mathrm{i} / \mathrm{o}}$ & $\mathrm{G} \alpha_{\mathrm{i} / \mathrm{o}}$ & $\mathrm{G} \alpha_{\mathrm{i} / \mathrm{o}}$ & $\mathrm{G} \alpha_{\mathrm{i} / \mathrm{o}}$ \\
\hline \multirow{5}{*}{$\begin{array}{l}\text { Primary signal } \\
\text { transduction }\end{array}$} & $\mathrm{cAMP} \downarrow$ & $\mathrm{cAMP} \downarrow$ & $\mathrm{cAMP} \downarrow$ & $\mathrm{cAMP} \downarrow$ & $\mathrm{cAMP} \downarrow$ \\
\hline & VOCC $\downarrow$ & $\mathrm{VOCC} \downarrow$ & $\mathrm{VOCC} \downarrow$ & $\mathrm{VOCC} \downarrow$ & $\mathrm{VOCC} \downarrow$ \\
\hline & GIRK $\uparrow$ & GIRK $\uparrow$ & $\mathrm{GIRK} \uparrow$ & $\mathrm{GIRK} \uparrow$ & $\mathrm{GIRK} \uparrow$ \\
\hline & NHE1 $\downarrow$ & $\mathrm{PTP} \uparrow$ & NHE1 $\downarrow$ & NHE1 1 & $\mathrm{PTP} \uparrow$ \\
\hline & $\mathrm{PTP} \uparrow$ & & $\mathrm{PTP} \uparrow$ & $\mathrm{PTP} \uparrow$ & \\
\hline \multirow{6}{*}{$\begin{array}{l}\text { Expression in human } \\
\text { normal tissue }\end{array}$} & Brain & Brain & Brain & Brain & \\
\hline & $\begin{array}{l}\text { Anterior pituitary } \\
\text { Pancreatic islets }\end{array}$ & Anterior pituitary & Anterior pituitary & Retina & Anterior pituitary \\
\hline & Gastrointestinal tract & Pancreatic islets & Pancreatic islets & Dorsal root ganglia & Pancreatic islets \\
\hline & & Dorsal root ganglia & Gastrointestinal tract & Placenta & $\begin{array}{l}\text { Gastrointestinal } \\
\text { tract }\end{array}$ \\
\hline & & Gastrointestinal tract & Lymphatic tissue & & Lymphatic tissue \\
\hline & & $\begin{array}{l}\text { Lymphatic tissue } \\
\text { Adrenals }\end{array}$ & Adrenals & & Adrenals \\
\hline \multirow{4}{*}{$\begin{array}{l}\text { Expression in human } \\
\text { tumors }^{a}\end{array}$} & GH-Adenomas & GH-Adenomas & GH-Adenomas & & GH-Adenomas \\
\hline & NET & TSH-Adenomas & ACTH-Adenomas & & ACTH-Adenomas \\
\hline & & NET & NF-Adenomas & & NET \\
\hline & & $\begin{array}{l}\text { Pheochromocytomas } \\
\text { Paragangliomas }\end{array}$ & & & \\
\hline \multirow[t]{3}{*}{$\begin{array}{l}\text { Phenotype of mice } \\
\text { lacking receptor }\end{array}$} & $\begin{array}{c}\text { Altered insulin } \\
\text { homeostasis }\end{array}$ & $\begin{array}{l}\text { High basal acid } \\
\text { secretion }\end{array}$ & $\begin{array}{l}\text { Impaired novel object } \\
\text { recognition }\end{array}$ & $\begin{array}{l}\text { Increased seizure } \\
\text { susceptibility }\end{array}$ & $\begin{array}{l}\text { Increased insulin } \\
\text { secretion }\end{array}$ \\
\hline & & Inhibition of glucagon & & Increased anxiety & Basal \\
\hline & & $\begin{array}{l}\text { Impaired motor } \\
\text { coordination }\end{array}$ & & & \\
\hline
\end{tabular}

${ }^{a}$ Expression in $>50 \%$ of cases. 
TABLE 2

Sequences of human receptors were aligned using the BLAST algorithm, and the percentages of sequence identity (upper right) and similarity (i.e., the presence of similar amino acids; lower left) were determined

Sequence comparisons are limited to the core regions of receptors (i.e., sequences encompassing the seven-helix bundle plus adjacent segments), whereas no significant similarities were detected in the $\mathrm{N}$-terminal and C-terminal tails.

\begin{tabular}{lrrrrr}
\hline & $\mathrm{SST}_{1}$ & $\mathrm{SST}_{2}$ & $\mathrm{SST}_{3}$ & $\mathrm{SST}_{4}$ & $\mathrm{SST}_{5}$ \\
\hline $\mathrm{SST}_{1}$ & 100 & 55 & 52 & 69 & 49 \\
$\mathrm{SST}_{2}$ & 74 & 100 & 53 & 53 & 56 \\
$\mathrm{SST}_{3}$ & 69 & 69 & 100 & 48 & 56 \\
$\mathrm{SST}_{4}$ & 82 & 73 & 66 & 100 & 53 \\
$\mathrm{SST}_{5}$ & 64 & 74 & 69 & 70 & 100 \\
\hline
\end{tabular}

in mammals, but is identifiable in several fish species. An additional tetraploidization in teleost fish gave rise to even more receptor species (Ocampo Daza et al., 2012; Tostivint et al., 2014). The common ancestor also gave rise to two so-called Drostar receptors in Drosophila (Kreienkamp et al., 2002) that are not activated by known mammalian peptides (including SRIF variants and opioids). However, their endogenous ligand allatostatin $\mathrm{C}$ bears only superficial similarity to SRIF. In contrast, the signature motif YANSCANPILY present in mammalian receptors is only slightly modified to YSNSAVNPILY in Drostar1, and the C-terminal PDZ ligand motif found in all SSTs is also present in the fly (Kreienkamp et al., 2002).

Genes encoding SSTs are intronless within their coding sequence, except for $\mathrm{SST}_{2}$. The $\mathrm{SST}_{2}$ gene can be alternatively spliced to produce two receptor proteins, $\mathrm{SST}_{2 \mathrm{~A}}$ and $\mathrm{SST}_{2 \mathrm{~B}}$, that differ in length and sequence of their carboxyl termini. Human tissues contain the unspliced $\mathrm{SST}_{2 \mathrm{~A}}$ variant exclusively, whereas both spliced forms have been identified in rodents (Vanetti et al., 1992). Although the $\mathrm{SST}_{5}$ gene does not contain CD introns, variants of $\mathrm{SST}_{5} \mathrm{mRNA}$ formed by splicing of noncanonical donor and acceptor splice sites are identified in humans, pigs, and rodents (Durán-Prado et al., 2009). The human $\mathrm{SST}_{5}$ variants encode truncated receptors containing five ( $\left.\mathrm{SST}_{5} \mathrm{TMD} 5\right)$ or four ( $\left.\mathrm{SST}_{5} \mathrm{TMD} 4\right)$ transmembrane domains and distinct carboxyl-termini (Durán-Prado et al., 2009).

Despite the prominent therapeutic role of $\mathrm{SST}_{2}$ and $\mathrm{SST}_{5}$-targeting SRIF analogs in pharmacotherapy of endocrine tumors, surprisingly few disease-associated mutations have been identified in any of the seven genes comprising the SRIF system (two peptide precursors and five receptor genes). To date, it has been reported that a single acromegaly patient resistant to octreotide treatment displayed a coding polymorphism in $\mathrm{SST}_{5}$ that clearly affected receptor signaling (Ballare et al., 2001). The R240W mutation presumably disrupts G protein and mitogen-activated protein kinase (MAPK) signaling, abolishing the antisecretory effects of SRIF on $\mathrm{SST}_{5}$-expressing cells. Besides this unique case, loss of heterozygosity at $\mathrm{SST}_{5}$ was speculated to lead to reduced mRNA expression, but molecular mechanisms for this notion have not been conclusively elucidated
(Lania et al., 2008). Although numerous studies have reported reduced $\mathrm{SST}_{2}$ and $\mathrm{SST}_{5}$ expression in treatmentresistant tumors, correlations with any particular polymorphism in SST genes have not been established. Molecular mechanisms underlying low SST expression in octreotide- or lanreotide-resistant tumors must therefore reside in genes outside of the SRIF system and still await identification.

Data from large-scale human sequencing studies, such as the exome aggregation consortium (which includes about 60,000 healthy individuals), further show that all five genes coding for SSTs are rather tolerant to sequence variations. In a ranking of about 18,000 human genes based on the presence of missense mutations, the genes coding for SST receptors are placed between positions $1459\left(\mathrm{SST}_{1}\right)$ and $9488\left(\mathrm{SST}_{5}\right)$, where 1 would be the gene that has the lowest tolerance for missense mutations (Lek et al., 2016).

The best-characterized action of SRIF is its strong inhibitory effect on both endocrine and exocrine cell secretion (Konturek et al., 1976; Dolais-Kitabgi et al., 1979; Mandarino et al., 1981). SRIF also inhibits neuronal excitability. All SST subtypes mediate inhibitory actions by association with $G_{i} / G_{o}$ proteins, members of the heterotrimeric guanine-nucleotidebinding protein family characterized by sensitivity to pertussis toxin (PTX) (Demchyshyn et al., 1993; Gu et al., 1995a,b; Gu and Schonbrunn, 1997; Carruthers et al., 1999). Activation of $\mathrm{G}_{\mathrm{i}} / \mathrm{G}_{\mathrm{o}}$ proteins by SSTs leads to suppression of two critical second messengers: cAMP and cytosolic $\mathrm{Ca}^{2+}$. The reduction in cAMP results from inhibition of adenylyl cyclase. SSTs act to inhibit calcium channels both directly and indirectly, the latter by opening $\mathrm{G}$ protein-activated inward-rectifier $\mathrm{K}$ channels (Kir3.x) to produce hyperpolarization and, as a consequence, inhibition of $\mathrm{Ca}^{2+}$ influx through voltage-operated calcium channels (VOCC) (Gromada et al., 2001). Reduction of either cAMP or cytosolic $\mathrm{Ca}^{2+}$ leads to inhibition of secretion, and the simultaneous reduction of both second messengers by SRIF results in synergistic inhibitory effects on hormone release. Signaling events responsible for inhibition of cell proliferation are less well understood than those that inhibit hormone secretion. One such pathway involves activation of protein tyrosine phosphatases (PTP) (Pan et al., 1992), including the Src homology region 2 domain-containing phosphatase (SHP)-1 and SHP-2. Ensuing dephosphorylation of specific substrates may counteract growth factor-stimulated tyrosine kinase activity and thus inhibit multiple mitogenic signaling pathways (Table 1).

Before subtype-specific antibodies became available, detailed mapping of receptor mRNA and binding sites had been described and comprehensively reviewed (Epelbaum et al., 1994; Patel, 1999; Dournaud et al., 2000). SSTs are widely expressed in the CNS and the endocrine system with some overlapping distributions, but different cellular and subcellular localizations 
(Piwko et al., 1996; Thoss et al., 1996a; Lanneau et al., 2000). Within the CNS, SSTs are mainly expressed on neurons in the cortex, hippocampus, amygdala, and hypothalamus (Perez et al., 1994; Perez and Hoyer, 1995; Thoss et al., 1996b; Hannon et al., 2002). In the endocrine system, SSTs are expressed on distinct cell populations in the anterior pituitary, pancreatic islets, adrenals, and neuroendocrine cells of the GIT. SSTs are also expressed on enteric ganglion and immune cells (Table 1).

All five SSTs bind the endogenous SRIF and CST ligands with high affinity. Many peptidic SRIF analogs have been developed, leading not only to the discovery of metabolically stable multireceptor SRIF analogs but also to subtype-selective receptor agonists and antagonists. Selective nonpeptide agonists and antagonists are now available for all five SSTs, except $\mathrm{SST}_{4}$, for which selective antagonists are still lacking (Table 1).

\section{Somatostatin Receptor 1}

\section{A. Somatostatin Receptor 1 Structure}

Human $\mathrm{SST}_{1}$ was the first SST cDNA to be cloned by Yamada et al. (1992a). Cloning was accomplished from a pool of GPCR-like sequences amplified from human pancreatic islet RNA by reverse-transcriptase polymerase chain reaction using a generic set of primers covering highly conserved amino acid sequences in the third and sixth GPCR transmembrane segments (Libert et al., 1989). The mouse homolog was then obtained by screening a mouse genomic library with a human $\mathrm{SST}_{1}$ probe (Yamada et al., 1992a). There is 99\% amino acid identity between human and mouse $\mathrm{SST}_{1}$ sequences. Meyerhof et al. (1991) had previously reported the sequence of a rat cDNA encoding a novel putative GPCR expressed primarily in the hypothalamus and cerebral cortex, but whose ligand could not be identified. The high identity of the predicted amino acid sequence of this orphan receptor (97\% and $98 \%$ identity with human and mouse $\mathrm{SST}_{1}$, respectively) indicated that it corresponded to the rat homolog.

In humans, $\mathrm{SST}_{1}$ is a 391-amino-acid protein encoded on chromosome $14 \mathrm{q} 13$ by an intronless gene whose promoter region, transcription start site, and $5^{\prime}$ untranslated region (UTR) have been elucidated (Fig. 3) (Redmann et al., 2007). Characterization of rabbit mAbs against $\mathrm{SST}_{1}$ (UMB-7) indicated that the protein is heavily glycosylated, migrating in SDS-PAGE at $M_{r}$ 45,000-60,000, but displaying the expected mol. wt. of $M_{r}$ 45,000 after protein extracts had been subjected to enzymatic deglycosylation (Lupp et al., 2013). The gene coding for mouse and rat $\mathrm{SST}_{1}$ is localized on chromosomes $12 \mathrm{C} 1$ and $6 \mathrm{q} 23$, respectively, and both encode a 391-amino-acid protein.

\section{B. Somatostatin Receptor 1 Signaling Mechanisms}

Studies on $\mathrm{SST}_{1}$ signaling in various cell types have yielded heterogeneous results, depending on whether $\mathrm{SST}_{1}$ is expressed endogenously or heterologously by transfection, indicating that the cellular environment is important in determining the signal transduction machinery. Signal transduction studied in $\mathrm{SST}_{1}$ heterologous cell systems was first thought to be G proteinindependent (Rens-Domiano et al., 1992; Buscail et al., 1994), but later shown to involve both PTX-sensitive and -insensitive G proteins (Garcia and Myers, 1994; Hadcock et al., 1994; Hershberger et al., 1994; Hou et al., 1994; Patel et al., 1994). Development of a

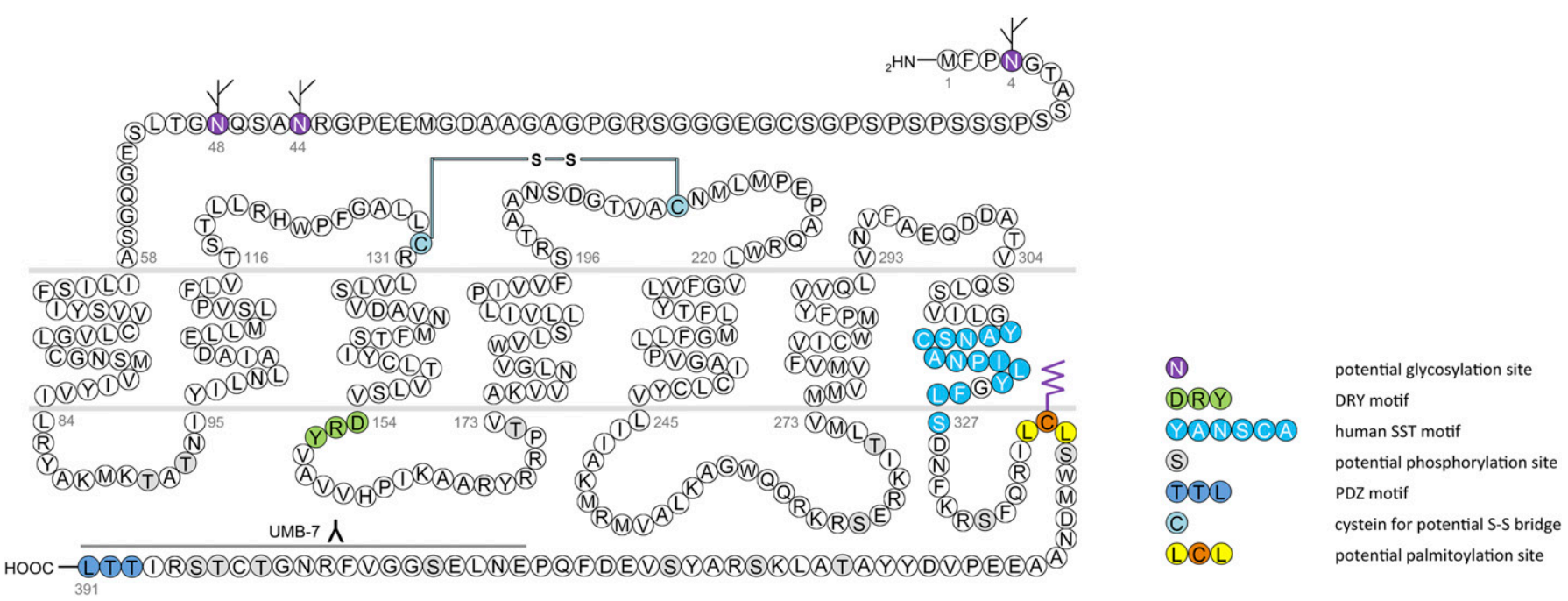

Fig. 3. Structure of human $\mathrm{SST}_{1}$. The primary and secondary amino acid structure of the human $\mathrm{SST}_{1}$ (UniProtKB - P30872) is shown in a schematic serpentine format. Glycosylation sites are colored in purple; the DRY motif is highlighted in green; the human SST motif is in light blue; potential phosphorylation sites are in gray; the PDZ ligand motif is in dark blue; the disulfide-forming cysteines are in pale blue; and the potential palmitoylation site is in orange. UMB-7 is a rabbit monoclonal antibody, which detects the carboxyl-terminal tail of SST 1 in a phosphorylationindependent manner. 
polyclonal antibody to a 15 -amino-acid peptide corresponding to a unique sequence in the $\mathrm{SST}_{1}$ carboxyl terminus has made it possible to immunoprecipitate endogenously expressed $\mathrm{SST}_{1}$ from pituitary tumor cell lysates and to demonstrate specific coupling to PTXsensitive $\mathrm{G}$ proteins ( $\mathrm{Gu}$ et al., 1995a). Gi proteins reported to couple to $\mathrm{SST}_{1}$ in $\mathrm{SST}_{1}$-transfected cells include $\mathrm{Gi}_{\alpha 1,2,3}$ (Hadcock et al., 1994; Kubota et al., 1994), although only $\mathrm{Gi}_{\alpha 3}$ dominantly couples $\mathrm{SST}_{1}$ to downstream adenylate cyclase inhibition (Kubota et al., 1994). $\mathrm{SST}_{1}$ transduces reduction of both cAMP accumulation and intracellular $\mathrm{Ca}^{2+}$ concentrations in heterologous cell systems (Fig. 4) (Garcia and Myers, 1994; Hadcock et al., 1994; Hershberger et al., 1994; Patel et al., 1994), as well as in insulinoma cells expressing $\mathrm{SST}_{1}$ endogenously (Roosterman et al., 1998). All SSTs regulate ion channels, including potassium channels (ATP-sensitive, inward, and delayed rectifying), as recently shown using an elegant fluorescence-based membrane potential assay in pituitary cells (Günther et al., 2016). $\mathrm{SST}_{1}$ activation results in membrane hyperpolarization and subsequent reduction of $\mathrm{Ca}^{2+}$ influx through voltage-sensitive $\mathrm{Ca}^{2+}$ channels, as demonstrated in endogenously $\mathrm{SST}_{1}$-expressing insulinoma cells (Roosterman et al., 1998). Interestingly, $\mathrm{SST}_{1}$, but not the other SSTs endogenously expressed in the mouse pancreatic $\beta$-cell line MIN6, shows exclusive coupling with $\mathrm{N}$-type voltage-sensitive $\mathrm{Ca}^{2+}$ channels, resulting in reduced intracellular $\mathrm{Ca}^{2+}$ concentrations and in inhibition of insulin secretion (Smith, 2009). Such SST specificities were also observed in pituitary tumor cells, where, in contrast to $\mathrm{SST}_{2}, \mathrm{SST}_{1}$ fails to stimulate phosphoinositide-specific phospholipase C (PLC) activity or PLC-dependent release of $\mathrm{Ca}^{2+}$ from intracellular stores (Chen et al., 1997), but transduces inhibition of phospholipase A2 activity and arachidonic acid release, similar to $\mathrm{SST}_{2}$ (Cervia et al., 2002). Additionally, $\mathrm{SST}_{1}$ (like $\mathrm{SST}_{3}$ or $\mathrm{SST}_{4}$, but not $\mathrm{SST}_{2}$ or $\mathrm{SST}_{5}$ ) inhibits sodium/hydrogen exchanger 1 (NHE1) activity via a PTX-independent mechanism, as demonstrated in $\mathrm{SST}_{1}$-transfected cells (Hou et al., 1994), resulting in decreased extracellular acidification (Chen and Tashjian, 1999) that may be involved in inhibition of cell migration by SRIF (Buchan et al., 2002). Hence, $\mathrm{SST}_{1}$, but not $\mathrm{SST}_{2}$, attenuated rat sarcoma (Ras) homolog (Rho)-GTP levels and subsequent Rock activity induced both by GPCR or integrin activation when expressed in Chinese hamster lung fibroblast cells (CCL39), and these inhibitory effects correlated with decreased actin stress fiber assembly and cell migration

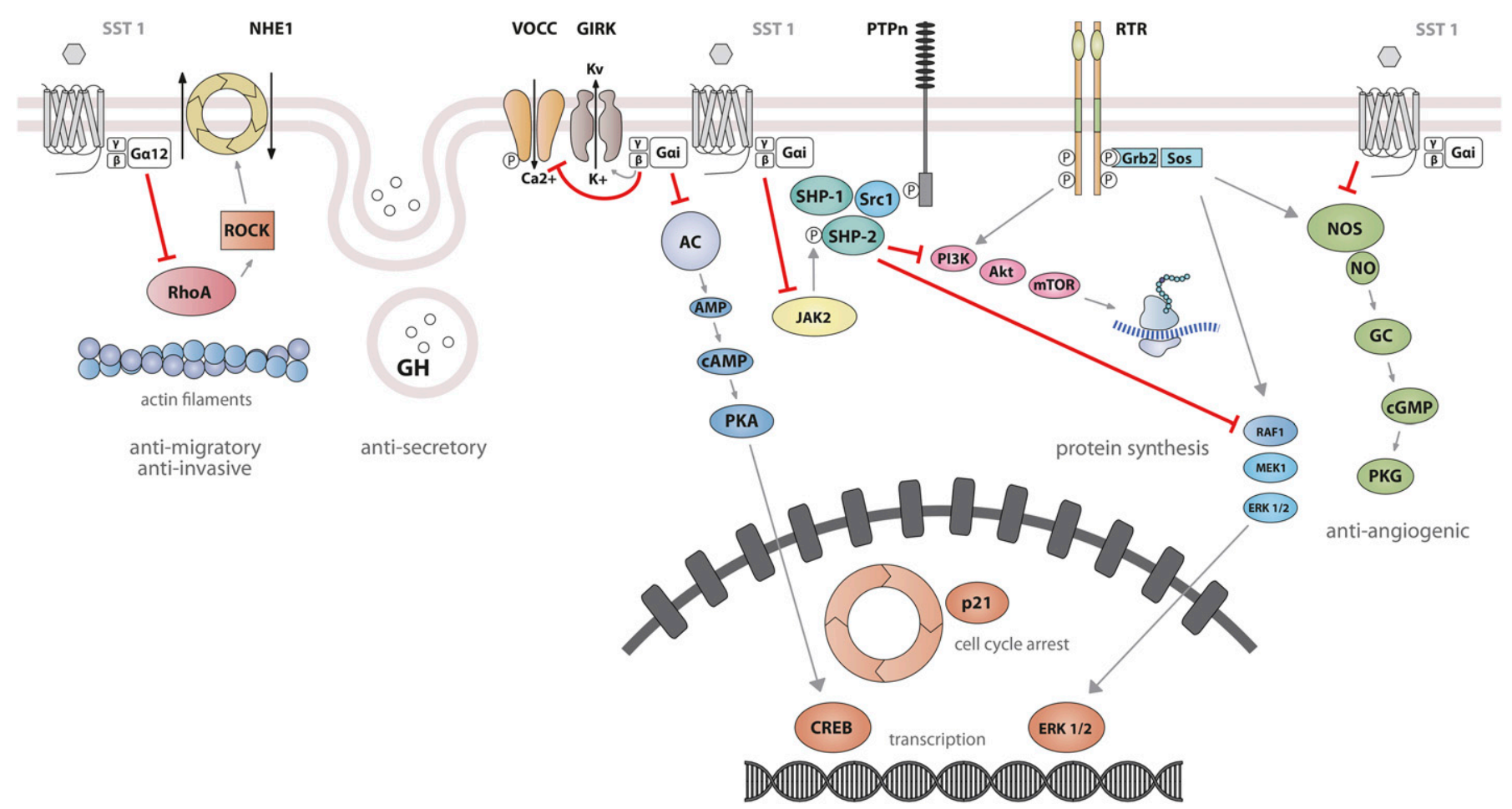

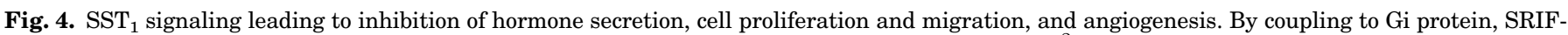

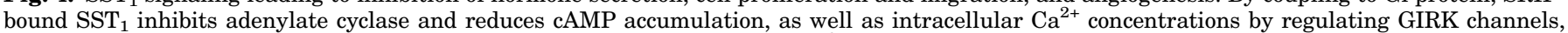

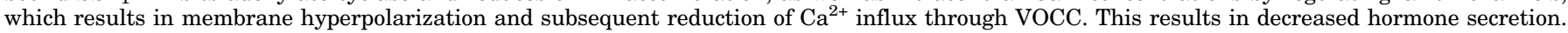

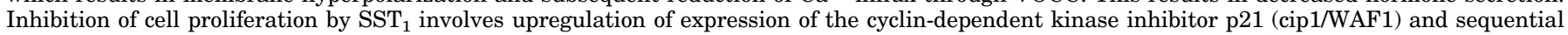

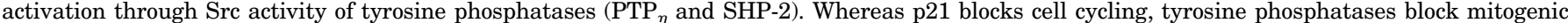

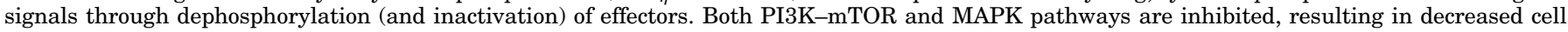

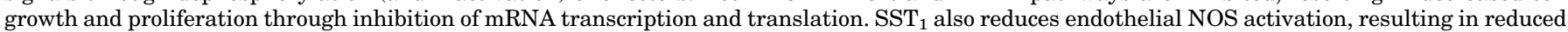

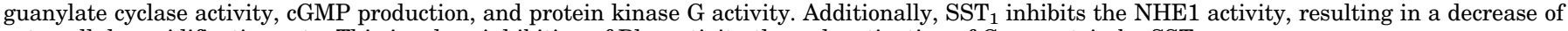
extracellular acidification rate. This involves inhibition of Rho activity through activation of $\mathrm{G}_{\alpha 12} \mathrm{protein}$ by $\mathrm{SST}_{1}$. 
(Buchan et al., 2002). Interestingly, a reported substrate of Rock is NHE1, which can serve as a plasma membraneanchoring scaffold for actin filaments to control assembly of cortical stress fibers and focal adhesions. Because Rho inhibition by $\mathrm{SST}_{1}$ is PTX-independent, it may involve activation of $\mathrm{G}_{\alpha 12}$, a trimeric $\mathrm{G}_{\alpha}$ protein reported to inhibit both NHE1 (Lin et al., 1996) and the Rho guanine nucleotide exchange factor p115 RhoGEF (Hart et al., 1998). Notably, NHE1 localizes at the invadopodia (membrane structures involved in cell invasion) of human malignant breast carcinoma cells, where it generates extracellular acidification necessary for invadopodial-dependent extracellular matrix degradation and tumor invasion (Busco et al., 2010).

SRIF-induced increase of PTP activity (Hierowski et al., 1985; Liebow et al., 1989; Pan et al., 1992) was shown to be mainly involved in SRIF inhibitory effects on growth factor-stimulated cell growth (Buscail et al., 1994; Florio et al., 1994, 1996). PTP activity was found in a membrane complex containing SRIF and SSTs (Zeggari et al., 1994; Srikant and Shen, 1996). In pituitary tumor cells, SRIF-induced activation of protein phosphatases via PTX-sensitive G proteins (White et al., 1991; Duerson et al., 1996) correlates with endogenous $\mathrm{SST}_{1}$ expression (Florio et al., 1994). The rat membrane-associated protein tyrosine phosphatase $\eta$ $\left(\mathrm{PTP}_{\eta}\right)$ (homolog to human receptor tyrosine phosphatase type J, formerly known as DEP-1) transduces SRIF antiproliferative effects, in both insulin and/or TSHtreated thyroid PC C13 cells (which express all SSTs but predominantly $\mathrm{SST}_{1}$ ), and in glioblastoma cells (Massa et al., 2004; Barbieri et al., 2008), which express all five SSTs (Mawrin et al., 2004). In $\mathrm{SST}_{1}$-expressing heterologous cell systems, PTP-dependent inhibition of cell proliferation by SRIF was reported to rely on a complex interplay of different PTPs, comprising the receptorlike $\mathrm{PTP}_{\eta}$, which provides a long-lasting PTP activity ( $>2$ hours), and the cytosolic SHP-2, which is rapidly activated (1-5 minutes) (Arena et al., 2007). SHP-2 and $\mathrm{PTP}_{\eta}$ are sequentially activated in a complex comprising the Janus kinase 2 (JAK2) that phosphorylates and activates SHP-2, which in turn activates (by dephosphorylation) the SRC proto-oncogene, nonreceptor tyrosine kinase (Src) that tyrosine phosphorylates and activates $\mathrm{PTP}_{\eta}$. The latter phosphatase is directly responsible for SRIF-mediated inhibitory effect on fibroblast growth factor (FGF)-stimulated proliferation through $\mathrm{SST}_{1}$ (Arena et al., 2007). SRIF-activated PTPs inhibit cell proliferation by dephosphorylating tyrosine kinase receptors and/or downstream effectors, such as plateletderived growth factor receptor, as demonstrated in $\mathrm{SST}_{1}$ expressing pancreatic cancer-associated fibroblasts (Duluc et al., 2015), or by inducing cell cycle arrest via upregulation of $\mathrm{p} 21$ (cip1/Waf1) expression in $\mathrm{SST}_{1^{-}}$ transfected cells (Florio et al., 1999). In addition to $\mathrm{SST}_{3}, \mathrm{SST}_{1}$ and $\mathrm{SST}_{2}$ blunt FGF-induced nitric oxide production through inhibition of endothelial nitric oxide synthase (NOS) in Chinese hamster ovary (CHO) cells in a PTX-dependent manner (Arena et al., 2005).

\section{Somatostatin Receptor 1 Regulation and Trafficking}

Sequence analyses of the rat Sstr1 gene promoter (Baumeister and Meyerhof, 1998, 2000a) demonstrated presence of putative transcription factor binding sites [GC box transcription factor, specificity protein 1, and activator protein (AP)-2] that are often found in TATAless promoters (Smale et al., 1990). Presence of binding sites for tissue-specific transcriptional factors of the POU domain protein family (Rosenfeld, 1991) was also noted, including sites for pituitary-specific positive transcription factor 1 and POU family transcription factor Tst-1 that regulate tissue-specific rat $S$ str 1 gene expression in the pituitary and in pancreatic $\beta$-cells, respectively (Baumeister and Meyerhof, 1998, 2000b). The porcine Sstr1 gene promoter showed positive regulation by cAMP (through a CREBBP1 binding site) (Gahete et al., 2014), consistent with the cAMP-mediated upregulation of $\mathrm{SST}_{1}$ mRNA in rat pituitary primary cultures induced by GHRH treatment (Park et al., 2000), and in pituitary adenomas expressing a mutated $\mathrm{G}_{\alpha \mathrm{s}}$ (gsp oncogene) that constitutively activates the cAMP pathway (Kim et al., 2005). $\mathrm{SST}_{1}$ mouse pituitary expression may also be controlled by testosterone because pituitary $\mathrm{SST}_{1}$ mRNA levels are decreased in gonadectomized males but restored upon testosterone injection, and are increased by testosterone treatment in rat pituitary tumor cells (GH4C1 cells) (Xu et al., 1995a; Senaris et al., 1996). Such Sstr1 gene regulation reported in mouse, rat, or pig was also confirmed for the human SSTR1 gene (Redmann et al., 2007). Finally, the $\mathrm{SST}_{1}$ gene promoter contains two CpG islands (Redmann et al., 2007), putatively involved in head and neck squamous cell carcinoma tumorigenesis, where hypermethylation of the SSTR1, but also of SRIF, gene has been correlated with reduced disease-free survival (Misawa et al., 2015).

SRIF binding to its receptors results in internalization of receptor-ligand complexes, a critical process for receptor downregulation, resensitization, and signaling (Tulipano and Schulz, 2007). Intriguingly, SST internalization may differ across species, explaining controversial results reported for $\mathrm{SST}_{1}$. In the rat insulinoma cell line 1046-38, which endogenously expresses $\mathrm{SST}_{1}$, a recombinant rat epitope-labeled $\mathrm{SST}_{1}$ was expressed to demonstrate that $\mathrm{SST}_{1}$ endocytosis is observed upon cell treatment with SRIF (Roosterman et al., 1997). This was also confirmed in other rat $\mathrm{SST}_{1}$-expressing heterologous cells (Roth et al., 1997b). Interestingly, ligand-induced rat $\mathrm{SST}_{1}$ trafficking was dynamic, involving endocytosis followed by recycling, and then re-endocytosis of the receptor and of the intact and biologically active ligand, which are not directed to lysosomal degradation (Roosterman et al., 1997). In contrast, other studies showed that 
human $\mathrm{SST}_{1}$ expressed in heterologous cell systems demonstrates very slow, if any, internalization upon ligand binding (Stroh et al., 2000a; Liu and Schonbrunn, 2001), although it undergoes acute desensitization of adenylyl cyclase coupling that correlates with its phosphorylation status (Hukovic et al., 1996; Liu and Schonbrunn, 2001). Differences in rat and human $\mathrm{SST}_{1}$ internalization may be due to species-specific trafficking. Responsible for this species effect might be an amino acid change at a putative phospho-acceptor site $\left(\mathrm{Thr}^{383}\right.$ $\mathrm{Cys}^{384}{ }_{-} \mathrm{Thr}^{385}{ }_{-} \mathrm{Ser}^{386}$ ) in the rat versus human $\mathrm{SST}_{1}$ C-terminal tail, where human $\mathrm{Ser}^{386}$ has been replaced by alanine in rat $\mathrm{SST}_{1}$. This substitution might explain the reported differences between rat and human $\mathrm{SST}_{1}$ affinity for $\beta$-arrestin- 1 and subsequent trafficking (Tulipano et al., 2004; Ramirez et al., 2005). Confocal microscopy analyses showed bright immunoreactivity of both human and rat $\mathrm{SST}_{1}$ within the cytoplasm, both receptors accumulating the ligand (SRIF-14) into superficial compartments. Intriguingly, a fraction of $\mathrm{SST}_{1}$ stays clustered immediately beneath the plasma membrane, in as yet unidentified intracellular vesicular compartments (Nouel et al., 1997; Roosterman et al., 1997, 2007; Hukovic et al., 1999). This peculiar localization may be caused by the absence of a domain in the $\mathrm{SST}_{1} \mathrm{~N}$ terminus required for cell surface targeting, as described for $\mathrm{SST}_{3}$ (Ammon et al., 2002). The $\mathrm{SST}_{1}$ cytoplasmic pool serves as a reservoir for short-term upregulation of human $\mathrm{SST}_{1}$ expression at the membrane upon prolonged agonist treatment. Upregulation depends on phosphorylation events at the $\mathrm{SST}_{1}$ C-terminal tail (Hukovic et al., 1999). $\mathrm{SST}_{1}$ immunoreactivity is observed both at the membrane and in the cytoplasm in primary and heterologous cell models (Gahete et al., 2014), and in paraffin-embedded sections of diverse human tumor tissues, in contrast to $\mathrm{SST}_{2}$, which is predominantly membrane-associated (Hofland et al., 1999; Lupp et al., 2013).

\section{Somatostatin Receptor 1 Interacting Proteins}

Unlike other SSTs, $\mathrm{SST}_{1}$ is not capable of homodimerization, prevented by structures within the $\mathrm{C}$-terminal domain (Grant et al., 2004). $\mathrm{SST}_{1}$ was nevertheless found heterodimerized with $\mathrm{SST}_{5}$ in $\mathrm{SST}_{1^{-}}$and $\mathrm{SST}_{5^{-}}$ coexpressing heterologous cell systems. Heterodimerization is induced by $\mathrm{SST}_{5}$ - but not $\mathrm{SST}_{1}$-selective ligands and changes intracellular signaling (inhibition of forskolin-stimulated cAMP production) of the $\mathrm{SST}_{1} / \mathrm{SST}_{5}$ heterodimer as compared with $\mathrm{SST}_{5}$ homodimers or $\mathrm{SST}_{1}$ monomers (Grant et al., 2004). SST 1 was also found heterodimerized with $\mathrm{SST}_{2}$ in prostate cancer cells; this complex stabilized with a bispecific $\left(\mathrm{SST}_{1} / \mathrm{SST}_{2}\right) \mathrm{SRIF}$ agonist, which nevertheless was less efficient than a mono-specific $\mathrm{SST}_{1}$ agonist to produce inhibition of cell proliferation (Ruscica et al., 2010). $\mathrm{SST}_{1}$, like other SSTs, harbors within its $\mathrm{C}$ terminus a PDZ-binding motif that interacts with membrane-associated guanylate kinase homologs, including PSD-95 and PSD-93 (Christenn et al., 2007), or synapse-associated protein SAP-97 (Cai et al., 2008), involved in SRIF signaling to regulate neuronal growth cone stability in neurons (including retraction of filopodia and lamellipodia). Whereas members of the membrane-associated guanylate kinase homolog subfamily are believed to play a role as molecular scaffolds in the organization of postsynaptic signaling machineries, SAP97 is also prominently expressed in axons and presynaptic terminals, where it may be involved in $\mathrm{SST}_{1}$ presynaptic functions (Cai et al., 2008).

\section{E. Somatostatin Receptor 1 Anatomic Framework}

Binding studies using iodinated SRIFs in mice deficient for each of the SSTs suggest that $\mathrm{SST}_{2}$ is most abundant in the murine CNS (Hannon et al., 2002; Videau et al., 2003), although expression of other SST subtypes, including $\mathrm{SST}_{1}$, was confirmed by in situ hybridization in rat brain (Beaudet et al., 1995; Stumm et al., 2004). Immunohistochemistry studies demonstrated that $\mathrm{SST}_{1}$ is highly expressed in the hypothalamic paraventricular and ARC, the median eminence (Helboe et al., 1998; Hervieu and Emson, 1998; Stroh et al., 2006), as well as other brain regions, including basal ganglia, basal forebrain regions, and hippocampus (Schulz et al., 2000a). SST 1 has also been localized in SRIF-containing amacrine cells of rat and rabbit retina (Dal Monte et al., 2003; Mastrodimou and Thermos, 2004). $\mathrm{SST}_{1}$ immunoreactivity is also intense in the spinal cord, especially in dorsal horn and dorsal medulla (Schulz et al., 2000a). Peripherally, $\mathrm{SST}_{1}$ is expressed in neurons of mouse, rat, and human dorsal root ganglia (DRG) (Bär et al., 2004; Imhof et al., 2011), and on intestinal mucosal nerve fibers (Van Op den Bosch et al., 2007). Outside the nervous system, high expression of human $\mathrm{SST}_{1}$ mRNA is apparent in stomach, intestine, and endocrine pancreas (Fig. 5) (Yamada et al., 1992a). Immunohistochemistry studies later confirmed that the $\mathrm{SST}_{1}$ protein is expressed mainly in these locations and also in the anterior pituitary (Portela-Gomes et al., 2000; Taniyama et al., 2005; Unger et al., 2012; Lambertini et al., 2013; Lupp et al., 2013). $\mathrm{SST}_{1}$ immunoreactivity is also positive in the parathyroid and bronchial glands (Taniyama et al., 2005), testis (staining in single cells between the tubules, resembling Leydig cells), and skeletal muscles (Unger et al., 2012). In the GI tract, $\mathrm{SST}_{1}$ is found expressed in stomach (Fig. 5), including antrum and corpus, in single cells resembling neuroendocrine or enterochromaffin-like cells (Unger et al., 2012), although expression in enterochromaffin cells (chromogranin-positive) was not always confirmed (Taniyama et al., 2005). Further $\mathrm{SST}_{1}$ expression was noted in the rectum (Taniyama et al., 2005) and also in enteric ganglion cells and corresponding nerve fibers and nerve terminals (Lupp et al., 2013). $\mathrm{SST}_{1}$ immunoreactivity was also detected in endothelial cells of blood 

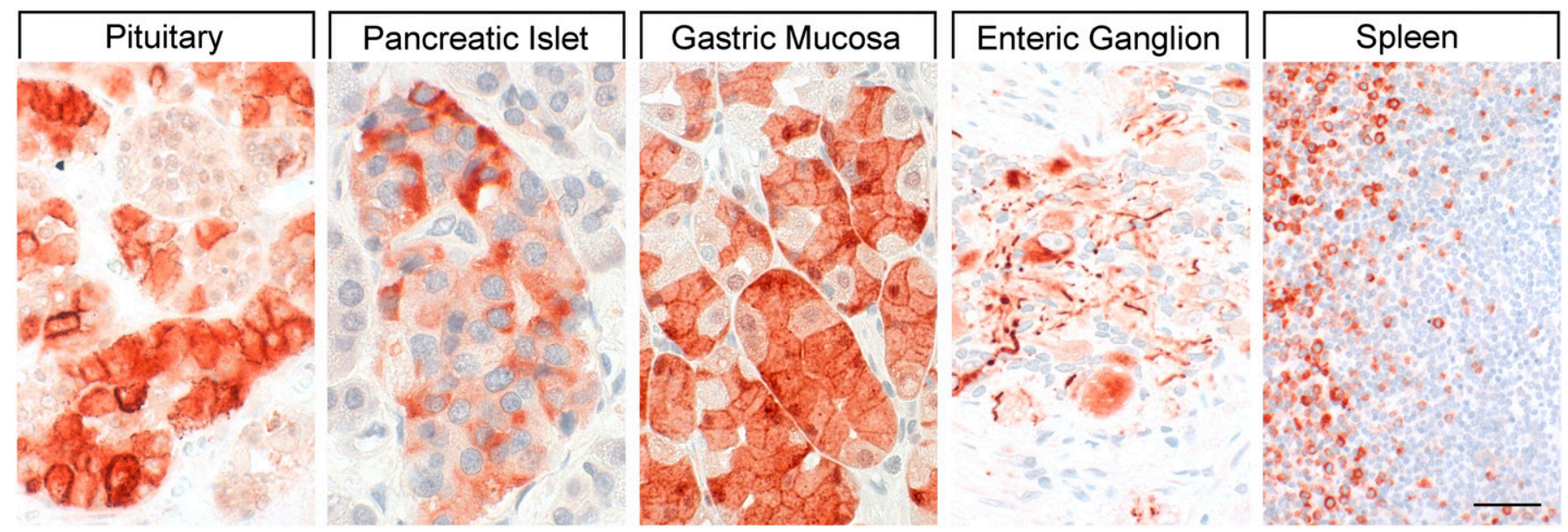

Fig. 5. $\mathrm{SST}_{1}$ expression pattern in normal human tissues. Immunohistochemistry (red-brown color), counterstaining with hematoxylin; primary antibody: UMB-7; scale bar, $50 \mu \mathrm{m}$. SST 1 displays both membranous and cytoplasmic expression.

vessels (Taniyama et al., 2005). In the immune system, $\mathrm{SST}_{1}$ protein is expressed in lymphocytes and macrophages (Taniyama et al., 2005), consistent with the positive $\mathrm{SST}_{1}$ immunoreactivity observed in cells resembling macrophages (cluster of differentiation 68 (CD68) positive) in spleen, lymph nodes, tonsils, thymus, lung, and gut mucosa, as well as in the stroma of diverse neoplasms (Lupp et al., 2013).

In neoplastic tissues, receptor autoradiography with $\mathrm{SST}_{1}$ subtype-selective ligands showed preferential expression of $\mathrm{SST}_{1}$ in prostate carcinomas and sarcomas (Reubi et al., 2001). Using the rabbit mAb UMB-7 (Table 3), $\mathrm{SST}_{1}$ expression was confirmed in prostate adenocarcinomas, rhabdomyosarcoma and liposarcoma, pituitary somatotroph adenomas, pancreatic adenocarcinoma, stomach cancer, urinary bladder cancer, pheochromocytoma, GI neuroendocrine tumors (NETs), breast carcinoma, cervix carcinoma, and ovarian tumors (Lupp et al., 2013). $\mathrm{SST}_{1}$ was abundantly expressed in bronchopulmonary NETs (Herrera-Martinez et al., 2017b) and positively associated with patient survival (Kaemmerer et al., 2015a).

\section{F. Somatostatin Receptor 1 Function}

In the $\mathrm{CNS}, \mathrm{SST}_{1}$ immunoreactivity is primarily found in fibers and terminals morphologically similar to varicose axons and that exhibit the highest brain SRIF immunoreactivity or are closely apposed by SRIFimmunoreactive fibers. This observation suggests that $\mathrm{SST}_{1}$ is predominantly targeted to presynaptic compartments (Schulz et al., 2000a). In this position it negatively modulates release of SRIF itself or of hypothalamic releasing and release-inhibiting hormones, including GHRH in ARC neurons, where high $\mathrm{SST}_{1}$ mRNA concentrations are found (Tannenbaum et al., 1998). Accordingly, $\mathrm{SST}_{1}$ was defined as an inhibitory autoreceptor located on the mediobasal hypothalamus, basal ganglia, and retina SRIF neurons (Thermos et al., 2006). Negative regulation of GHRH release by SRIF is consistent with the reported $\mathrm{SST}_{1}$ inhibitory role on hypothalamic regulation of GH secretion (Kreienkamp et al., 1999; Lanneau et al., 2000). Intriguingly, genderrelated differences in both number and labeling density of $\mathrm{SST}_{1}$ mRNA-expressing cells are observed in the rat ARC (i.e., two- to threefold increase in males versus females) (Zhang et al., 1999). This observation may explain lower basal GH levels in male than in female mammals (Jansson et al., 1985), and also the sexually dimorphic GH pulsatile secretion (Low et al., 2001). More recently, the negative regulation by SRIF of GHRH neuron electrical activity was decrypted using a GHRH-GFP transgenic model (Osterstock et al., 2016). It revealed a sexual dimorphism, which is primarily attributable to a sex-dependent control of GABAergic and glutamatergic inputs by SRIF, rather than intrinsic differences in the GHRH neurons themselves. Interestingly, the positive glutaminergic neurotransmission onto GHRH neurons is an obligatory target of SRIF in female, providing a mechanism for a more tonic inhibition in female than in male, where this inhibitory signal was absent in one-third of animals. Intriguingly, this is the opposite for SRIF inhibition of

TABLE 3

Rabbit monoclonal SST antibodies

\begin{tabular}{|c|c|c|c|c|}
\hline & Clone & Epitope & Species Reactivity & Reference \\
\hline $\mathrm{SST}_{1}$ & UMB-7 & ${ }^{377}$ ENLESGGVFRNGTCTSRITTL ${ }^{391}$ & Human & Lupp et al. (2013) \\
\hline $\mathrm{SST}_{2}$ & UMB-1 & ${ }^{355}$ ETQRTLLNGDLQTSI $^{369}$ & Mouse, rat human & Fischer et al. (2008) \\
\hline $\mathrm{SST}_{3}$ & UMB-5 & ${ }^{398}$ QLLPQEASTGEKSSTMRISYL $^{418}$ & Human & Lupp et al. (2012) \\
\hline $\mathrm{SST}_{5}$ & UMB-4 & ${ }^{344}$ QEATPPAHRAAANGLMQTSKL ${ }^{364}$ & Human & Lupp et al. (2011) \\
\hline
\end{tabular}


GABAergic (negative) inputs, being especially robust and synchronized in males. Both $\mathrm{SST}_{1}$ and $\mathrm{SST}_{2}$ are involved in GHRH neuron rhythmicity, but $\mathrm{SST}_{1}$ receptors specifically transduce SRIF inhibitory control of GABAergic inputs, likely taking place at the presynaptic level (Osterstock et al., 2016). Seven percent of neuropeptide $\mathrm{Y}$-positive neurons in the ARC coexpress $\mathrm{SST}_{1}$ mRNA, suggesting a direct interaction between the somatotropic axis and neuroendocrine regulatory loops of energy homeostasis (Fodor et al., 2005). Hypothalamic paraventricular and $\mathrm{ARC} \mathrm{SST}_{1}$ may account for prevention of acute stress-induced gut motor functions in mice after central injection of a $\mathrm{SST}_{1}$-selective agonist, including inhibition of gastric emptying and stimulation of colonic motility (Stengel et al., 2011), putatively through central SRIFergic regulation of corticotropin-releasing factor (CRF) release and downstream stress-induced CRF actions (Stengel et al., 2013). Recently, neuroanatomical connections between somatostatin and kisspeptin neurones were observed in the rat $\mathrm{ARC}$ and ventromedial hypothalamus, where one-third of kisspeptin neurones exhibit $\mathrm{SST}_{1}$ immunoreactivity. Because kisspeptin is a gonadotropinreleasing hormone secretagogue, these observations suggest that the regulation of kisspeptin release by $\mathrm{SST}_{1}$ may at least be partly involved in the well-known inhibition of gonadotropin-releasing hormone release by SRIF (Dufourny et al., 2018). In the basal ganglia (substantia nigra, nucleus accumbens, globus pallidus, and ventral pallidum), $\mathrm{SST}_{1}$ is also present presynaptically, where it negatively regulates SRIF release (Vasilaki et al., 2004), strongly suggesting that it may serve as an autoreceptor to modulate systems regulated by SRIF (including dopamine). $\mathrm{SST}_{1}$ (together with $\mathrm{SST}_{2}$ ) are abundantly expressed in nerve processes of basal forebrain regions, including substantia innominata and the horizontal limb of the diagonal band (Hervieu and Emson, 1998), where SRIF inhibits glutamate release presynaptically through $\mathrm{SST}_{1}$, thereby regulating excitability of forebrain cholinergic neurons (Momiyama and Zaborszky, 2006). Expression and function of $\mathrm{SST}_{1}$ in the rodent hippocampus have been controversial until the demonstration of $\mathrm{SST}_{1}$-mediated SRIF inhibitory action on synaptic transmission, using hippocampal slices of $\mathrm{SST}_{1} \mathrm{KO}$ mice and a $\mathrm{SST}_{1}$ selective agonist (Cammalleri et al., 2009). Hippocampal activity is regulated by $\mathrm{SST}_{1}$ through presynaptic inhibition of glutamate release induced by epileptiform treatment. In the spinal cord, $\mathrm{SST}_{1}$ may be involved in nociceptive transmission because dorsal horn and medulla regions coexpress $\mathrm{SST}_{1}$ together with SRIF, which has analgesic effects in rodents and humans (Malcangio, 2003; Imhof et al., 2011). In the peripheral nervous system, $\mathrm{SST}_{1}$ may be involved in inhibitory effects of SRIF on inflammation and nociception (Pinter et al., 2006), such as in mouse models of stress-related visceral nociception (Mulak et al., 2015) or immune-mediated arthritis (Imhof et al., 2011). In the retina, activation of $\mathrm{SST}_{1}$ with a selective ligand decreases SRIF release from retinal explants (Mastrodimou and Thermos, 2004). Surprisingly, loss of $\mathrm{SST}_{1}$ expression in $\mathrm{SST}_{1} \mathrm{KO}$ mice results in upregulated SRIF and $\mathrm{SST}_{2}$ retinal expression, together with an enlargement of axonal terminals of rod bipolar cells, where $\mathrm{SST}_{2}$ is expressed, as well as enhanced $\mathrm{SST}_{2}$ function (Bigiani et al., 2004; Pavan et al., 2004). Conversely, in $\mathrm{SST}_{2} \mathrm{KO}$ mice, $\mathrm{SST}_{1}$ expression is upregulated and rod bipolar cell axonal terminals are smaller (Casini et al., 2004). This suggests reciprocal inhibitory retinal roles of $\mathrm{SST}_{1}$ on $\mathrm{SST}_{2}$ expression, and vice versa. It certainly contributes to SRIFergic regulation of glutamatergic transmission along the vertical retinal visual pathway in which the $\mathrm{SST}_{2} / \mathrm{SRIF}$ receptor/ligand pair is probably restrained by $\mathrm{SST}_{1}$, consistent with reported $\mathrm{SST}_{1}$ autoreceptor functions (Dal Monte et al., 2003; Thermos et al., 2006).

In the periphery, $\mathrm{SST}_{1}$ is expressed, together with $\mathrm{SST}_{5}$ (Strowski et al., 2003), in a high percentage of pancreatic $\beta$-cells (Portela-Gomes et al., 2000), consistent with its reported role in regulating insulin secretion in studies using SST-selective agonists or in $\mathrm{KO}$ mouse models (Wang et al., 2004; Smith, 2009). In the anterior pituitary, the $\mathrm{SST}_{1}$-selective agonist $\mathrm{CH}-275$ decreases $\mathrm{GH}$ secretion in wild-type, but not primary somatotroph cultures derived from $\mathrm{SST}_{1}-\mathrm{KO}$ mice (Kreienkamp et al., 1999), demonstrating the critical role for $\mathrm{SST}_{1}$ in regulating pituitary $\mathrm{GH}$. $\mathrm{SST}_{1}$ was expressed in endothelial cells of normal human veins and arteries, including atherosclerotic arteries. $\mathrm{SST}_{1^{-}}$ selective agonists demonstrated consistent angioinhibitory effects in vitro (Bocci et al., 2007) and induced vascular relaxation through cytoskeletal alterations (Liapakis et al., 1996), making $\mathrm{SST}_{1}$-specific analogs interesting for treatment of vascular diseases, including intimal hyperplasia. Intriguingly, another study localized $\mathrm{SST}_{1}$ mRNA and $\mathrm{SST}_{1}$ protein to vascular smooth muscle cells, where it showed acute upregulation of expression during vascular trauma coincidently with smooth muscle cell proliferation, making this receptor an interesting target to inhibit myointimal proliferation (Khare et al., 1999). The presence of $\mathrm{SST}_{1}$ in intestinal macrophages and mast cells, especially during inflammation, has been described in mice (Perez et al., 2003; Van Op den Bosch et al., 2007). Low $\mathrm{SST}_{1}$ expression was also found in macrophages differentiated from peripheral bone marrow cell-derived monocytes, where it mediates together with $\mathrm{SST}_{2}$ anti-inflammatory effects after activation by a multireceptor SRIF analog (Armani et al., 2007). During liver inflammation, such as in cirrhosis or hepatocellular carcinoma (HCC), all five SST mRNAs were expressed, whereas expression was not observed in normal human liver. The specific $\mathrm{SST}_{1}$ agonist L-797,591 was the only SST agonist to inhibit both liver cancer cell and hepatic stellate cell migration, making $\mathrm{SST}_{1}$ agonists putatively interesting 
to treat liver cirrhosis or HCC (Reynaert et al., 2004). Accordingly, SRIF also reduced production of collagens and inflammatory cytokines by hepatic stellate cells, although the specific receptor subtype was not identified. This putatively explains antifibrotic and immunomodulatory actions of SRIF in the liver (Lang et al., 2005; Reynaert et al., 2005). $\mathrm{SST}_{1}$ was also found uniquely expressed in stellate cells of pancreatic adenocarcinoma, and its activation by pasireotide reduced chemoprotective and prometastatic features of these fibroblastic cells by reducing IL- 6 and collagen- 1 secretion (Duluc et al., 2015; Moatassim-Billah et al., 2016).

$\mathrm{SST}_{1}$ is overexpressed in prostate cancer (Sinisi et al., 1997; Kosari et al., 2008) and mediates antiproliferative effects and inhibition of prostate-specific antigen release induced by the $\mathrm{SST}_{1}$-selective agonist BIM-23926 in prostate cancer cell lines (Pedraza-Arevalo et al., 2017). In the thymus, $\mathrm{SST}_{1}$ mRNA is expressed on isolated thymic epithelial cells, where SRIF inhibits proliferation (Ferone et al., 1999). $\mathrm{SST}_{1}$ mRNA is not expressed in fresh human or rat thymocytes (Sedqi et al., 1996; Ferone et al., 2002), but rat thymocyte activation with phytohemagglutinin or IL-1 selectively induced $\mathrm{SST}_{1}$ (Sedqi et al., 1996), suggesting $\mathrm{SST}_{1}$ involvement in thymocyte proliferation and differentiation.

\section{G. Somatostatin Receptor 1 Ligands}

The lack of available SRIF analogs with selectivity for $\mathrm{SST}_{1}$ stimulated the search for such compounds. The first to be identified is $\mathrm{CH}-275$, which harbors a peptidic scaffold with selected amino acid deletions (des-aa1,2,5SRIF) that in combination with DTrp at position 8, and 4-(N-isopropyl)-aminomethylphenylalanine (IAmp) at position 9, yields des-aa1,2,5-[DTrp8,IAmp9]-SRIF (CH-275), a SRIF agonist with nanomolar affinity for $\mathrm{SST}_{1}$, and that was 30 -fold more selective for $\mathrm{SST}_{1}$ versus $\mathrm{SST}_{2} / 4 / 5$ and 10 -fold versus $\mathrm{SST}_{3}$, respectively (Liapakis et al., 1996). Using integrated combinatorial chemistry with high-throughput receptor-binding approaches, a $\mathrm{SST}_{1}$-selective nonpeptide compound (L-797,591) (Fig. 6; Table 4) displaying agonistic activity with an $\mathrm{IC}_{50}$ of $3 \mathrm{nM}$ was the first pharmacological tool identified for selective $\mathrm{SST}_{1}$ in vitro and in vivo studies (Rohrer et al., 1998). BIM-23926 is a synthetic $\mathrm{SST}_{1}$-selective agonistic peptide $\left(\mathrm{IC}_{50}\right.$ of $\left.4 \mathrm{nM}\right)$, reported to decrease cell viability of human medullary thyroid carcinoma TT cells, as well as to inhibit calcitonin release and cAMP levels (Fig. 6; Table 4) (Zatelli et al., 2002). SRA880 is the first reported nonpeptide SRIF $\mathrm{SST}_{1}$ competitive antagonist, with high affinity for both native and recombinantly expressed $\mathrm{SST}_{1}$ from various species (Fig. 6; Table 4) (rat, mouse, monkey, human), while displaying low affinity for a range of other neurotransmitter receptors, except the dopamine receptor D4 (Hoyer et al., 2004). The compound is bioavailable and brain penetrant. Consistent with the inhibitory autoreceptor role of $\mathrm{SST}_{1}$, SRA880 administration increases SRIF brain release and signaling, countering depressivelike symptoms in mice (Nilsson et al., 2012). A series of $\mathrm{SST}_{1}$-selective ergoline derivatives has been developed, some of which show effective oral bioavailability and brain penetration (Hurth et al., 2007; Troxler et al., 2008). The only clinically approved SRIF analog showing high affinity for $\mathrm{SST}_{1}$ is pasireotide, a nonselective peptidic compound displaying an $\mathrm{IC}_{50}$ of $9.3 \mathrm{nM}$ for $\mathrm{SST}_{1}$, and $\mathrm{IC}_{50}$ values for $\mathrm{SST}_{2}, \mathrm{SST}_{3}$, and $\mathrm{SST}_{5}$ of 1 , 1.5 , and $0.16 \mathrm{nM}$, respectively (Schmid, 2008).

\section{Somatostatin Receptor 2}

\section{A. Somatostatin Receptor 2 Structure}

cDNAs coding human and mouse $\mathrm{SST}_{2}$ were isolated, together with cDNAs coding for $\mathrm{SST}_{1}$, in early 1992 using a polymerase chain reaction-based approach with primers directed to regions conserved in all GPCRs (Yamada et al., 1992a). In the same year, a cDNA encoding for rat $\mathrm{SST}_{2}$ was identified by expression cloning from a rat brain cDNA library (Kluxen et al., 1992). The gene coding human $\mathrm{SST}_{2}$ is localized on chromosome 17q25.1 and consists of two exons. Whereas exon 1 contains only $5^{\prime}$ UTR, the entire coding region and 3'UTR are located on exon 2 . Genes encoding for mouse and rat $\mathrm{SST}_{2}$ are localized on chromosomes 11 E2 and 10q32.1, respectively. Homology between human and rodent $\mathrm{SST}_{2}$ is $94 \%$ (mouse) and $93 \%$ (rat) at the amino acid level, respectively. In all three species,

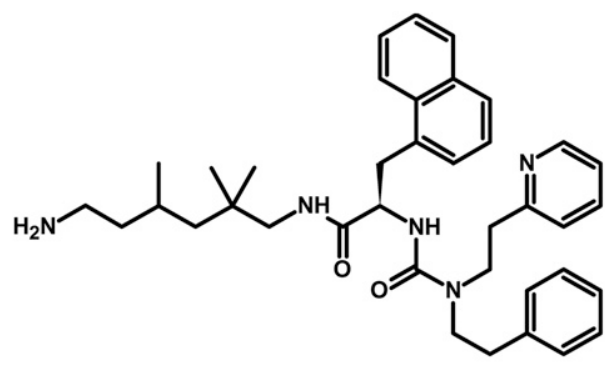

L-797,591

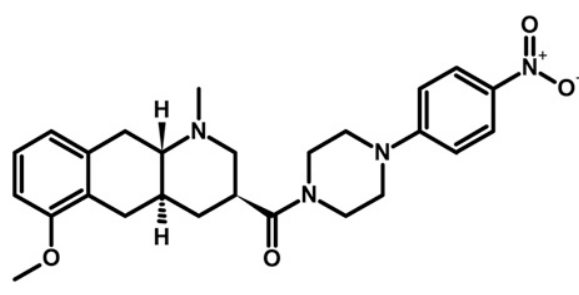

SRA880

Fig. 6. Structures of synthetic $\mathrm{SST}_{1}$ ligands. L-797,591, $\mathrm{SST}_{1}$ agonist; $\mathrm{SRA}_{880}, \mathrm{SST}_{1}$ antagonist. 
TABLE 4

Ligand-binding affinities of $\mathrm{SST}_{1}$-selective ligands

\begin{tabular}{lccccr}
\hline & $\mathrm{SST}_{1}$ & $\mathrm{SST}_{2}$ & $\mathrm{SST}_{3}$ & $\mathrm{SST}_{4}$ & $\mathrm{SST}_{5}$ \\
\hline $\mathrm{L}-797 / 591^{a}$ & 1.4 & 1875 & 2240 & 170 & 3600 \\
BIM-23926 $^{b}$ & 3.6 & $>1000$ & $>1000$ & 833 & 788 \\
SRA880 $^{c}$ & 7.6 & $>1000$ & $>1000$ & $>1000$ & 954 \\
\hline
\end{tabular}

${ }^{a}$ Data from Rohrer et al. (1998).

${ }^{b}$ Data from Zatelli et al. (2002).

${ }^{c}$ Data from Hoyer et al. (2004), Cammalleri et al. (2009).

$\mathrm{SST}_{2}$ is a 369 -amino-acid protein $\left(M_{r}=41,305\right.$ in humans), displaying typical seven-transmembrane segments and four putative $\mathrm{N}$-glycosylation sites (Asn-9, Asn-22, Asn-29, and Asn-32) (Fig. 7). In Western blot experiments, the protein is detected as a characteristic smear migrating between 70 and $80 \mathrm{kDa}$, in keeping with the assumption that these Asn residues are extensively glycosylated. In addition to this long receptor species, a mouse $\mathrm{SST}_{2}$ splice variant codes for a shortened receptor with an alternative C-terminal tail (termed $\mathrm{SST}_{2 \mathrm{~B}}$ ) (Vanetti et al., 1992). This variant arises due to removal of a part of the second exon that codes for the C-terminal tail of the long $\left(\mathrm{SST}_{2 \mathrm{~A}}\right)$ variant, and that also contains some $3^{\prime} \mathrm{UTR}$. Some of the remaining $3^{\prime} \mathrm{UTR}$ of $\mathrm{SST}_{2} \mathrm{~A}$ then becomes the coding region in $\mathrm{SST}_{2 \mathrm{~B}} \mathrm{mRNA}$; in contrast to $\mathrm{SST}_{2 \mathrm{~A}}$, the C-terminal sequence of $\mathrm{SST}_{2 \mathrm{~B}}$ is very poorly conserved between species. Several functionally relevant elements of the $\mathrm{SST}_{2 \mathrm{~A}} \mathrm{C}$ terminus are lost due to the alternative splicing event: 1) elimination of phosphorylation sites that contribute to agonistdependent desensitization and internalization and 2) $\mathrm{SST}_{2 \mathrm{~A}}$ contains a C-terminal consensus motif for binding PDZ domains (Zitzer et al., 1999), which is not present in in $\mathrm{SST}_{2} \mathrm{~B}$. Whereas both spliced forms have been identified in rodents (Vanetti et al., 1992), human tissues exclusively contain the unspliced $\mathrm{SST}_{2 \mathrm{~A}}$ variant. Consequently, we use the denomination $\mathrm{SST}_{2}$ for the long unspliced $\mathrm{SST}_{2 \mathrm{~A}}$ variant throughout this review.

\section{B. Somatostatin Receptor 2 Signaling Mechanisms}

Signaling properties of $\mathrm{SST}_{2}$ have been investigated in several heterologous expression systems, or in endogenous $\mathrm{SST}_{2}$-expressing cells using $\mathrm{SST}_{2}$ agonists. As physiologic actions of SRIF (e.g., on GH release) are sensitive to PTX (Cronin et al., 1983), unsurprisingly, $\mathrm{SST}_{2}$ also acts mostly through PTX-sensitive $\mathrm{G}$ proteins of the inhibitory/olfactory family of $\mathrm{G}_{\alpha}$ subunit $\left(\mathrm{G}_{\mathrm{i} / \mathrm{o}}\right)$ type (Law et al., 1993), as reported in cultured mammalian cells heterologously expressing $\mathrm{SST}_{2}$, such as CHO, human embryonic kidney (HEK)293 cells, or SV4transformed fibroblast-like derived monkey kidney (COS-7) cells. Major effects of $\mathrm{SST}_{2} / \mathrm{G}_{\alpha \mathrm{i} / \mathrm{o}}$ signaling are inhibition of adenylyl cyclase, inhibition of voltagegated calcium channels, and activation of $\mathrm{K}_{\mathrm{ir}} 3 . \mathrm{x}$ (Fig. 8) (Kreienkamp et al., 1997). The inability of SRIF-bound $\mathrm{SST}_{2}$ to inhibit cAMP production in some cell lines (e.g., Law et al., 1993) was ascribed to lack of expression of an appropriate G protein (Hershberger et al., 1994). All of these effects are complementary for the inhibition of excitable cells, such as neurons or hormone-secreting cells. Thus, whereas hormone secretion by pituitary cells is driven by $\mathrm{Ca}^{2+}$ influx through voltage-gated $\mathrm{Ca}^{2+}$ channels, activation of potassium channels by SRIF-activated $\mathrm{SST}_{2}$ hyperpolarizes the membrane and prevents depolarization induced by hypothalamic releasing hormones. In pituitary cells, $\mathrm{SST}_{2}$ activates PLC [more specifically the PLC- $\beta 3$ isozyme (Kim et al., 2012), which is partially blocked by PTX, putatively involving a $\mathrm{G}_{\alpha \mathrm{q} / 11}$ (Chen et al., 1997) and/or $\mathrm{G}_{\beta \gamma}$ protein (Kim et al., 2012)]. This results in inositol-1,4,5trisphosphate formation and $\mathrm{Ca}^{2+}$ release into the cytoplasm from the endoplasmic reticulum, regulating

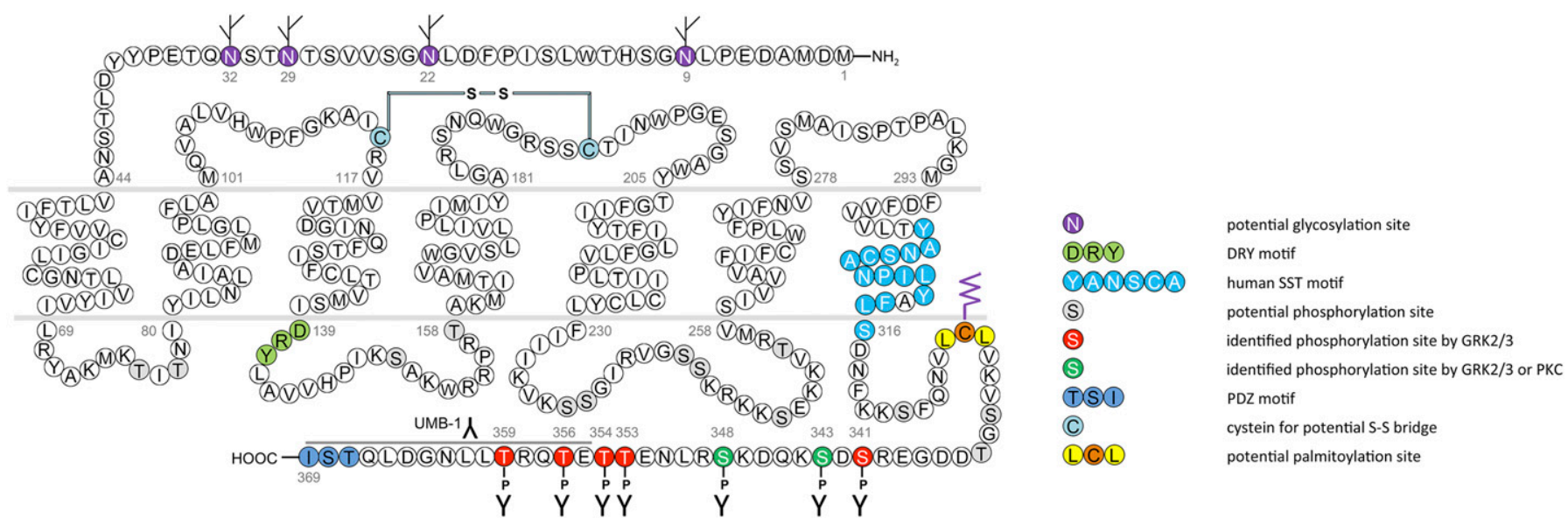

Fig. 7. Structure of human $\mathrm{SST}_{2}$. The primary and secondary amino acid structure of the human $\mathrm{SST}_{2}$ (UniProtKB - P30874) is shown in a schematic serpentine format. Glycosylation sites are colored in purple; the DRY motif is highlighted in green; the human SST motif is in light blue; potential phosphorylation sites are in gray; identified GRK2/3 phosphorylation sites are in red; identified GRK2/3 or PKC phosphorylation sites are in dark green; the PDZ ligand motif is in dark blue; the disulfide-forming cysteines are in pale blue; and the potential palmitoylation site is in orange. UMB-1 is a rabbit monoclonal antibody, which detects the carboxyl-terminal tail of $\mathrm{SST}_{2}$ in a phosphorylation-independent manner. 


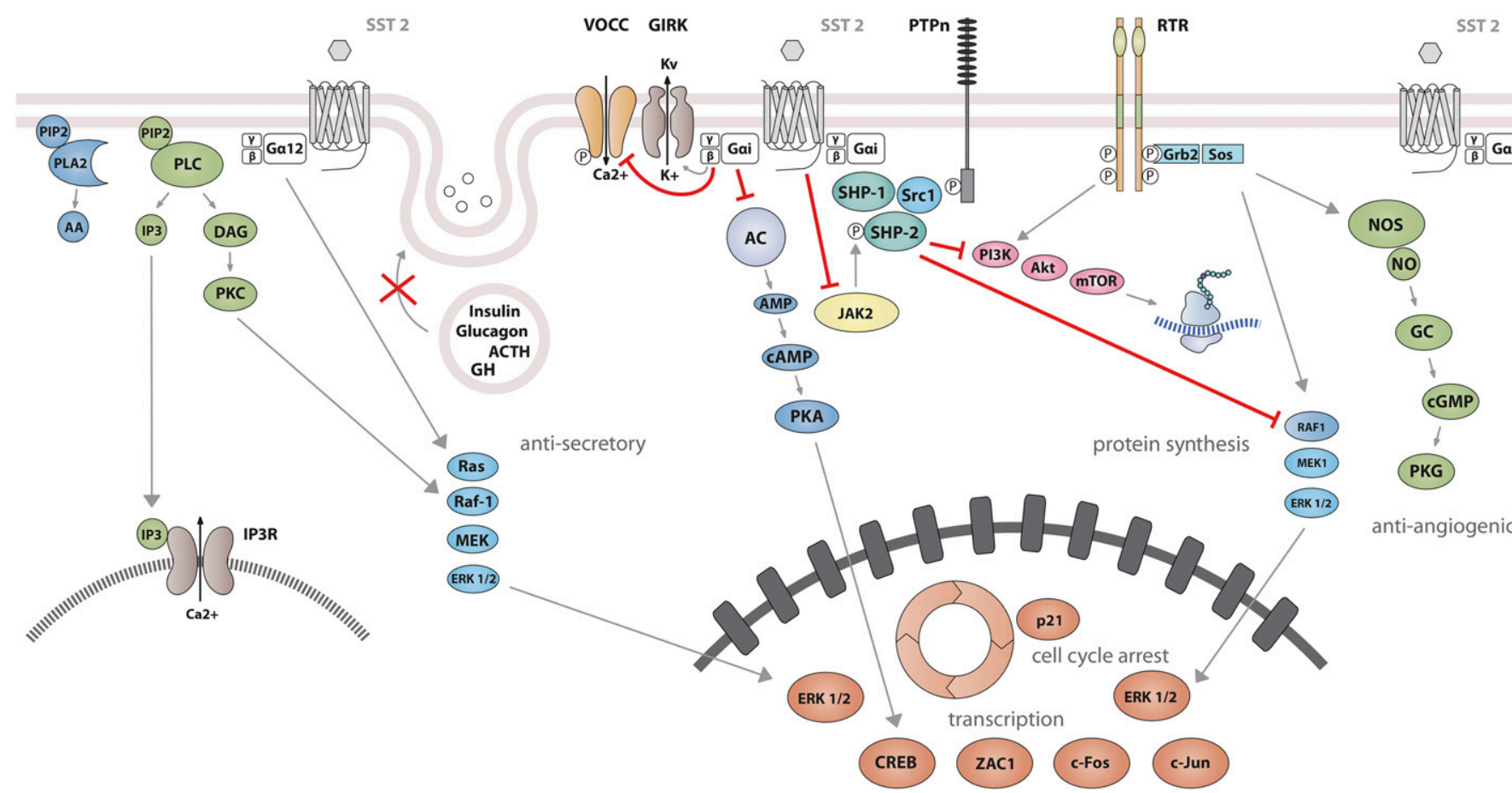

Fig. 8. $\mathrm{SST}_{2}$ signaling leading to inhibition of hormone secretion, cell proliferation and migration, and angiogenesis. By coupling to Gi proteins, $\mathrm{SST}_{2}$ inhibits adenylate cyclase and reduces cAMP accumulation, and reduces intracellular $\mathrm{Ca}^{2+}$ concentrations by activating GIRK channels, which results in membrane hyperpolarization and subsequent reduction of $\mathrm{Ca}^{2+}$ influx through VOCC. This results in decreased hormone secretion. By coupling to a pertussis toxin-independent $\mathrm{G}$ protein, $\mathrm{SST}_{2}$ activates $\mathrm{PLC}$, triggering inositol-1,4,5-trisphosphate $\left(\mathrm{IP}_{3}\right)$ production and subsequent $\mathrm{Ca}^{2+}$ release into the cytoplasm from the endoplasmic reticulum. Major downstream effectors of SST 2 are the tyrosine phosphatases SHP-1 and SHP-2 and the tyrosine kinase Src, which subsequently inhibit the PI3K-mTOR, MAPK, JAK2, and neuronal NOS pathways, thereby decreasing cell growth and proliferation. $\mathrm{SST}_{2}$-dependent inhibition of cell proliferation involves upregulation of the transcription factor ZAC1, triggering cell cycle inhibition

the MAPK/extracellular signal-regulated kinase (ERK) pathway (Kim et al., 2012).

Besides these canonical $G$ protein-mediated signaling pathways, $\mathrm{SST}_{2}$ also activates tyrosine phosphatase activity, associated with reduced serum-stimulated cell proliferation (Buscail et al., 1994; Reardon et al., 1997). This effect has been replicated in several cell types either overexpressing the receptor, or expressing lower endogenous levels of $\mathrm{SST}_{2}$ (Dent et al., 1997; Barbieri et al., 2008). Activation of tyrosine phosphatases by SRIF is PTXsensitive and can be mimicked by addition of $\mathrm{G}_{\alpha \mathrm{i} / \mathrm{o}}$ subunits purified from brain (Dent et al., 1997), implicating this $\mathrm{G}$ protein in the $\mathrm{SST}_{2}$-initiated signaling pathway. The nonreceptor tyrosine protein phosphatases SHP-1 and SHP-2, respectively, have emerged as major effectors (Lopez et al., 1997; Reardon et al., 1997), being copurified with $\mathrm{SST}_{2}$ (or SSTs in general) in heterologous cell systems, and sequentially activated, dependent on $\mathrm{G}$ protein $\left(\mathrm{G}_{\alpha \mathrm{i} 3}\right.$ and $\left.\mathrm{G}_{\beta \gamma}\right)$ and Src activity (Lopez et al., 1997; Ferjoux et al., 2003). Through phosphatase activation, $\mathrm{SST}_{2}$ inhibits tyrosine phosphorylation events such as those following activation of tyrosine kinase receptors (Bousquet et al., 1998; Hortala et al., 2003). This leads to cell cycle arrest and subsequent inhibition of cell proliferation, through upregulation of the cyclin-dependent kinase inhibitor p27/Kip1 (Pagès et al., 1999) and the zinc finger protein (Zac1) (Theodoropoulou et al., 2006), as mainly demonstrated in heterologous cell systems (Pagès et al., 1999; Grant et al., 2008). Furthermore, similar results were obtained using $\mathrm{SST}_{2}$-preferring or selective analogs in endogenously $\mathrm{SST}_{2}$-expressing cells [e.g., pituitary tumors (Ferrante et al., 2006; Hubina et al., 2006; Theodoropoulou et al., 2006; Horiguchi et al., 2009; Peverelli et al., 2017), insulinoma (Aoki et al., 2014), glioma (Barbieri et al., 2009), normal and tumoral pancreatic acinar cells (Charland et al., 2001), or thyroid cells (Medina et al., 1999)]. This involves regulation of several signaling pathways, including Ras/Raf/ERK (Dent et al., 1997; Lahlou et al., 2003), phosphatidylinositol-4,5-bisphosphate 3-kinase (PI3K)/AKT serine/ threonine kinase 1 (AKT)/glycogen synthase kinase $3 \beta /$ mechanistic target of rapamycin kinase (mTOR) (Bousquet et al., 2006; Theodoropoulou et al., 2006; Azar et al., 2008), p38 (Alderton et al., 2001), neuronal NOS (Lopez et al., 2001), and JAK2 (Hortala et al., 2003). Depending on phosphatase activity, $\mathrm{SST}_{2}$ activation also triggers apoptosis in endogenous $\mathrm{SST}_{2}$-expressing pituitary somatotroph tumor cells (Ben-Shlomo and Melmed, 2010), and also in pancreatic cancer cells engineered to express this receptor, where apoptosis is further stimulated by treatment with death ligands (Ben-Shlomo and Melmed, 2010). Finally, $\mathrm{SST}_{2}$-induced dephosphorylation events can also lead to inhibition of cell migration and invasion, through inhibition of 
the small $\mathrm{G}$ protein Rac and of the subsequent ruffle formation in endogenous $\mathrm{SST}_{2}$-expressing neuroblastoma cells (Pola et al., 2003). In addition, $\mathrm{SST}_{2}$-induced dephosphorylation events can lead to restoration of cellto-cell (adherens and gap junctions) and cell-to-matrix (hemidesmosomes) contacts in $\mathrm{SST}_{2}$-transfected pancreatic cancer cells (Benali et al., 2000; Lahlou et al., 2005; Laval et al., 2014).

\section{Somatostatin Receptor 2 Regulation}

Similar to genes encoding the other SSTs, genes coding $\mathrm{SST}_{2}$ from various species do not contain TATA and CAAT boxes (Greenwood et al., 1995). A minimal promoter fragment close to the transcription initiation site was identified as a novel initiator element sufficient to account for transcription from the $\mathrm{SST}_{2}$ promoter in neuroblastoma cells (Pscherer et al., 1996). Interestingly, a so-called enhancer box was identified in this region, which serves as a binding site for the basic helixloop-helix transcription factor (SEF-2). Interaction of SEF-2 with the enhancer box was identified as a major driving force for activity of the promoter in several cell lines (Pscherer et al., 1996). Transcriptional activity is further enhanced by the binding of c-myc intron binding protein 1 (MIBP1), both to SEF-2 and to a thyminecytosine-rich transcriptional enhancer element; the expression pattern of MIBP1 matches that of $\mathrm{SST}_{2}$ in the murine brain, suggesting that MIBP1 confers expression tissue specificity at least in the CNS (Dorflinger et al., 1999). In addition, negative regulatory elements have been identified in more distal regions of the promoter of the mouse Sstr2 gene. Suppression of transcriptional activity by this region may be overcome by Smad3/Smad4, which plates the activity of the Sstr2 gene under control of a transforming growth factor $\beta$-dependent signaling pathway (Puente et al., 2001). Finally, and relevant for tumors that escape $\mathrm{SST}_{2}$ mediated antiproliferative effect of SRIF and its analogs, an alternative $5^{\prime} /$ upstream promoter was identified that may be silenced by methylation (Torrisani et al., 2008). High methylation levels in this region correlated with reduced $\mathrm{SST}_{2}$ expression in tumor cells (Shen et al., 2016).

Regulation of $\mathrm{SST}_{2}$ depends upon molecular mechanisms, implying phosphorylation events at the C-terminal tail followed by recruitment of $\beta$-arrestins and receptor endocytosis. Combined biochemical and mutagenesis approaches identified serine and threonine residues in the C-terminal tail of the $\mathrm{SST}_{2}$ that are phosphorylated upon SRIF and/or octreotide stimulation, namely, S341, S343, S348, T353, and T354 in rat $\mathrm{SST}_{2}$-transfected CHO and GH4C1 cells (Liu et al., 2009), and S341, S343, T353, T354, T356, and T359 in HEK293 cells stably transfected with rat or human $\mathrm{SST}_{2}$ (Nagel et al., 2011; Lehmann et al., 2014b). Agonist-dependent phosphorylation of the four threonine residues was also documented in rat pituitary tumor cells (GH3) transiently transfected with rat $\mathrm{SST}_{2}$, rat pancreatic insulinoma $\beta$-cells (INS1 cells), which endogenously express $\mathrm{SST}_{2}$, and rat pancreas in vivo (Poll et al., 2010). Although rat $\mathrm{SST}_{2}$ internalization was partially inhibited by mutation of threonine residues, none of the mutations resulted in a complete block of receptor internalization (Liu et al., 2008). By contrast, multisite phosphorylation of clusters of carboxylterminal serine and threonine residues of the human $\mathrm{SST}_{2}$ cytoplasmic tail is a critical event for receptor endocytosis (Lehmann et al., 2014b). Accordingly, in a SRIFoma, which synthesized and secreted SRIF and in which $\mathrm{SST}_{2}$ are localized intracellularly, receptors are phosphorylated, whereas in an ileal carcinoid tumor in which $\mathrm{SST}_{2}$ are membrane-bound, receptors are found nonphosphorylated (Liu et al., 2003). In human NET samples, $\mathrm{SST}_{2}$ phosphorylation is observed only in octreotide-treated patients and receptors are internalized, whereas in untreated tumors $\mathrm{SST}_{2}$ are not phosphorylated and are located at the cell membrane (Waser et al., 2012). Of note, unlike SRIF and octreotide, pasireotide (formerly known as SOM230) stimulates only phosphorylation of S341 and S343 residues of human $\mathrm{SST}_{2}$, followed by a partial receptor internalization (Lesche et al., 2009; Lehmann et al., 2014b). In cell lines, G protein-coupled receptor kinase $\left(\mathrm{GRK}_{2}\right)$ (Liu et al., 2009) or $\mathrm{GRK}_{3}$ is involved in phosphorylation of S341 and S343 residues (Nagel et al., 2011), whereas the threonine residues (T353, T354, T356, and T359) are phosphorylated by $\mathrm{GRK}_{2}$ and $\mathrm{GRK}_{3}$ (Poll et al., 2010). In HEK293 cells stably expressing $\mathrm{SST}_{2}$, chemical protein phosphatase inhibitors and small interfering RNA knockdown screening lead to identification of protein phosphatase $1 \beta$ (PP1 $\beta)$ as the GPCR phosphatase that catalyzes rapid dephosphorylation of residues T353, T354, T356, and T359 (Poll et al., 2011).

$\mathrm{SST}_{2 \mathrm{~B}}$ terminates after residue 332 and therefore does not contain the phosphorylation sites identified in the C-terminal tail of $\mathrm{SST}_{2}$ (Cole and Schindler, 2000). Accordingly, $\mathrm{SST}_{2 \mathrm{~B}}$ phosphorylation is not detectable after agonist stimulation of colonic adenocarcinoma cells, whereas $\mathrm{SST}_{2}$ is phosphorylated under the same conditions (Holliday et al., 2007). SRIF causes rapid desensitization of $\mathrm{SST}_{2}$, but not of $\mathrm{SST}_{2 \mathrm{~B}}$, in this latter cell type. However, both receptor subtypes desensitized markedly in transfected CHO cell line subclone K1 (CHO-K1) cells (Cole and Schindler, 2000). Interestingly, phosphorylation in the third ICL of $\mathrm{SST}_{2}$, a sequence shared by the $\mathrm{SST}_{2 \mathrm{~B}}$ variant, also occurs (Hipkin et al., 2000; Elberg et al., 2002), but does not play a role in internalization and desensitization (Lehmann et al., 2014b). Differences in receptor phosphorylation might be physiologically relevant in the rodent brain and the GIT, where different expression patterns of the two $\mathrm{SST}_{2}$ subtypes have been documented (Cole and Schindler, 2000).

The relationship between $\mathrm{SST}_{2}$ phosphorylation and $\beta$-arrestin binding, a major class of adaptor proteins 
involved in GPCR desensitization and internalization, has been investigated in different cell lines. In HEK293 (Tulipano et al., 2004) and CHO cells (Liu et al., 2005), or primary hippocampal neurons (Lelouvier et al., 2008), cotransfected with $\beta$-arrestin enhanced green fluorescent protein and the rat $\mathrm{SST}_{2}$, both $\beta$-arrestin- 1 and $\beta$-arrestin- 2 are recruited to the plasma membrane after agonist stimulation, form stable complexes with the receptor, and internalize together. $\beta$-arrestin-2 recruitment also occurs after agonist stimulation of human $\mathrm{SST}_{2}$ in HEK293 cells (Lehmann et al., 2014b). Together, these results suggest that the $\mathrm{SST}_{2}$ belongs to the class B GPCR subgroup, because its activation results in robust recruitment of both $\beta$-arrestin- 1 and -2 (Oakley et al., 2000).

\section{Somatostatin Receptor 2 Trafficking}

Studying SST trafficking has received increasing attention because the fate of internalized receptors, following agonist exposure, may vary from degradation to rapid recycling to the plasma membrane, thereby affecting responsiveness to endogenous ligands and drugs of therapeutic interest. A striking $\mathrm{SST}_{2}$ property is that in the vast majority of the cell types endogenously expressing this subtype, it is almost exclusively confined to the plasma membrane, such as in central and myenteric neurons, neuroendocrine cells of the gastric antrum (Gugger et al., 2004; Fischer et al., 2008), anterior pituitary (Fischer et al., 2008; Peineau et al., 2014), pancreatic islets, as well as central and peripheral tumors (Reubi et al., 2000b). However, intracytoplasmic $\mathrm{SST}_{2}$ localization was observed in CNS in regions exhibiting dense SRIF innervation such as the central nucleus of the amygdala (Dournaud et al., 1998). Intracellular localization was also described in a rat model of middle cerebral artery occlusion in cerebrocortical neurons adjacent to the infarct, which regionally correlates with transient SRIF depletion from axonal terminals (Stumm et al., 2004). Subcellular distribution of the receptor may be dependent on surrounding SRIF concentrations, as suggested in tumors of the nervous and neuroendocrine systems (pheochromocytomas and neuroblastomas) producing autocrine SRIF (Reubi et al., 2000b). Collectively, these studies highlight that SRIF released under physiologic or pathophysiological conditions regulates localization and trafficking of $\mathrm{SST}_{2}$ consistent with results obtained in cell lines (Csaba and Dournaud, 2001; Tulipano and Schulz, 2007; Jacobs and Schulz, 2008; Treppiedi et al., 2017). In hippocampal neuronal cells, $\mathrm{SST}_{2}$ trafficking was analyzed in detail at different times after acute intracerebral octreotide injections or in primary neuronal culture exposed to SRIF ligands (Csaba et al., 2001, 2007; Lelouvier et al., 2008; De Bundel et al., 2015). These experiments demonstrated for the first time that GPCR cargoes recycle through the trans-Golgi network (TGN) after endocytosis. After activation and internalization, endosomes bearing $\mathrm{SST}_{2}$ in dendrites (by far the major pool of $\mathrm{SST}_{2}$ ) and cell bodies fuse and migrate to a perinuclear compartment expressing trans-Golgi markers such as the integral protein of the TGN, TGN38, and syntaxin-6, but not cis-golgi markers such as cis-Golgi marker 130 (GM130). These results have been comfirmed in vivo by electron microscopy approaches that showed that $\mathrm{SST}_{2}$ cargoes were not targeted to degradative departments; rather, TGN-enriched receptors recycle to the plasma membrane (dendrites and cell bodies), where they are observed in preagonist challenge equivalent amounts. The recycling process, which depends on the length and extension of dendritic arborization, is slow, 3-6 hours in vitro and 24-48 hours in vivo. Differences in kinetics between in vivo and in vitro studies might be, at least in part, due to the persistence of the intracerebrally injected agonist, which cannot be removed or chased as in in vitro settings, implying several internalization/ recycling cycles before total agonist clearance or degradation. Such trafficking of activated $\mathrm{SST}_{2}$ to the TGN was also reported in myenteric neurons, in which an intact TGN is necessary for receptor recycling (Zhao et al., 2013). In both DRGs and dorsal horn neurons, octreotideactivated $\mathrm{SST}_{2}$ in vivo also are observed to concentrate in perinuclear regions that resemble the TGN before recycling (24 hours) (Shi et al., 2014). The physiologic significance of this peculiar recycling pathway is not fully understood. Recycled receptor might undergo biochemical modifications and/or association with scaffolding proteins for proper delivery to the cell surface. An additional intriguing hypothesis is that $\mathrm{SST}_{2}$ targeted to the TGN could produce downstream cellular responses, such as coupling to different $\mathrm{G}$ proteins, as demonstrated for other GPCRs, the sphingosine 1-phosphate receptor (Mullershausen et al., 2009), and the TSH receptor (Calebiro et al., 2009).

Recently, studies have focused on factors involved in regulation of intracellular $\mathrm{SST}_{2}$ trafficking. Modulating recycling of a particular receptor can indeed impact its physiologic fate and therefore offer a potential therapeutic value. Using pharmacological and cell biologic approaches, it was demonstrated that leucyl-cysteinyl aminopeptidase (LNPEP; formerly known as insulinregulated aminopeptidase) ligands accelerate recycling of internalized $\mathrm{SST}_{2}$ in neurons in vitro or in vivo (De Bundel et al., 2015). LNPEP, which shares common regional and subcellular distribution with internalized $\mathrm{SST}_{2}$, was shown to be involved in vesicular trafficking (Wright and Harding, 2011). Importantly, because LNPEP ligands increase the density of $\mathrm{SST}_{2}$ at the plasma membrane, they also potentiate SRIF-inhibitory effects on seizure activity (De Bundel et al., 2015). LNPEP therefore represents a therapeutic target for treatment of limbic seizures and possibly for other neurologic conditions in which downregulation of GPCRs occurs. In myenteric neurons, activated $\mathrm{SST}_{2}$ traffic to endothelinconverting enzyme 1 (ECE-1)-containing vesicles and 
TGN (Zhao et al., 2013). This endosomal peptidase degrades peptide ligands in intracellular organelles and promotes receptor resensitization (Roosterman et al., 2007). $\mathrm{SST}_{2}$ recycling (30 minutes) in myenteric neurons is dependent upon endosomal acidification, ECE-1 activity, and an ECE-1 cleavable ligand, which is the case for SRIF-14, but not for SRIF-28 or analogs, such as octreotide (Zhao et al., 2013). Hence, after activation by ECE-1-resistant SRIF-28 and analogs, $\mathrm{SST}_{2}$ remain within the TGN and are poorly recycled at 120 minutes. Assuming that $\mathrm{SST}_{2}$ signals in intracellular organelles, this could explain, at least in part, the long-lasting actions of SRIF analogs such as octreotide. Of note, although ECE-1 might be present in the hippocampus (Barnes et al., 1997), the kinetics of $\mathrm{SST}_{2}$ recycling were the same after activation by SRIF-14 or octreotide in hippocampal neurons (De Bundel et al., 2015), suggesting that the ECE-1 role on $\mathrm{SST}_{2}$ recycling is dependent upon cell types. Filamin A (FLNA), a scaffolding protein involved in intracellular trafficking of several transmembrane proteins (Onoprishvili et al., 2003; Noam et al., 2014), has been shown to interact with the $\mathrm{SST}_{2}$ in melanoma and pancreatic cell lines (Najib et al., 2012). FLNA appears crucial for $\mathrm{SST}_{2}$ stabilization and signaling at the plasma membrane (Peverelli et al., 2014; Vitali et al., 2016). In addition, FLNA may protect $\mathrm{SST}_{2}$ from degradation by facilitating targeting of the receptor to a recycling pathway during long-term agonist treatment of pancreatic tumor and GH-secreting tumor cells (Peverelli et al., 2014; Vitali et al., 2016).

\section{E. Somatostatin Receptor 2 Interacting Proteins}

Interestingly, all SST subtypes ( $\mathrm{SST}_{1}-\mathrm{SST}_{5}$; with the exception of the short-splice variant $\mathrm{SST}_{2 \mathrm{~B}}$ ) contain a consensus motif for interaction with type I PDZ domains at the intracellular $\mathrm{C}$ termini. This motif is conserved throughout evolution, as the closest homologs of SSTs in Drosophila, the Drostar1 and Drostar2 receptors for type $\mathrm{C}$ allatostatins, also contain a PDZ ligand motif (Kreienkamp et al., 2002). In contrast, closely related opioid receptors are devoid of such a motif. In its simplest form, a type I PDZ ligand consists of the C-terminal sequence $-\mathrm{S} / \mathrm{T}-\mathrm{X}-\phi-\mathrm{COOH}$, in which $\phi$ is a large hydrophobic residue (Phe in $\mathrm{SST}_{4}$; Ile or Leu in $\mathrm{SST}_{1,2,3}$; Val in many other typical PDZ ligand motifs). Whereas such a motif is quite common, flanking sequences add to the specificity of PDZ-type interactions (Zeng et al., 2016), thus ensuring that not every PDZ ligand can interact with any PDZ domain. $\mathrm{SST}_{2}$ was the first SST for which an interaction with a PDZ domain-containing protein was reported; Zitzer et al. (1999) identified members of the Shank protein family as potential interactors through yeast two-hybrid screening (Zitzer et al., 1999). Shank proteins are important scaffold proteins of the PSD. They exhibit a complex domain structure, as the central PDZ domain is accompanied by a Ras association domain, a set of seven ankyrin repeats, and a sterile alpha motif (SAM domain) and a nuclear localization signals 1 domain. In addition, a long proline-rich stretch is involved in binding actin-binding proteins. In the PSD, Shank proteins are considered as master scaffold proteins that link receptor complexes to the actin-based cytoskeleton (Kreienkamp, 2008). As $\mathrm{SST}_{2}$ does not appear to be a postsynaptically enriched receptor, it appears likely that interactions between Shank proteins and $\mathrm{SST}_{2}$ are relevant at other, nonsynaptic sites. In further studies, PDZ domain-containing 1 (PDZ-K1) protein was identified as interaction partner for all SST subtypes, including $\mathrm{SST}_{2}$. As PDZ-K1 also interacts with a PLC isoform, this work suggests that PDZ-K1 allows for coupling of SST subtypes to PLC through ternary complex formation. Finally, C-terminal PDZ ligand motifs of a larger number of membrane proteins have been shown to promote postendocytic recycling through binding to the PDZ domain containing sorting nexin family member 27 (Steinberg et al., 2013), and the PDZ ligand of $\mathrm{SST}_{2}$ may also promote similar recycling.

Additionally, $\mathrm{SST}_{2}$ harbors two immunoreceptor tyrosine-based inhibition motif(ITIM) sequences (immunoreceptor tyrosine-based inhibitory motif: I/V/L/S-x-Y$\mathrm{x}-\mathrm{x}-\mathrm{L} / \mathrm{V})$, present in the third ICL and C-terminal tail (Ferjoux et al., 2003). Such ITIM consensus sequences were initially found in inhibitory immunoreceptors (e.g., programmed cell death protein 1), triggering $B$ cell receptor inhibition of SHP-2 recruitment, and subsequent dephosphorylation of $\mathrm{B}$ cell receptor effector molecules (Okazaki et al., 2001). Similarly, SRIF-induced phosphorylation of $\mathrm{SST}_{2}$ tyrosine 228 and 312 residues, present in each of the two $\mathrm{SST}_{2}$ ITIMs, triggers SHP-2 (but not SHP-1) direct recruitment to $\mathrm{SST}_{2}$, SHP-2 activation, and subsequent transduction of dephosphorylation events also involving the kinase Src and SHP-1, leading to cell proliferation inhibition (Ferjoux et al., 2003).

The first $\mathrm{SST}_{2}$ ICL contains two juxtaposed binding sites for the $\mathrm{p} 85$ regulatory subunit of PI3K and for the actin-binding and scaffolding protein FLNA (Bousquet et al., 2006; Najib et al., 2012). Depending on $\mathrm{SST}_{2}$ phosphorylation of tyrosine residues 66 and 71, present in FLNA and p85 binding sites, respectively, FNLA or p85 competitively binds to $\mathrm{SST}_{2}$ first ICL. In the absence of SRIF, these tyrosine residues are phosphorylated, enabling p85, but not FLNA, binding. This state is permissive for growth factor-induced activation of PI3K activity. In the presence of SRIF, $\mathrm{SST}_{2}$ tyrosine residues 66 and 71 are dephosphorylated, enabling FLNA, but not p85 binding, the dissociation of which from $\mathrm{SST}_{2}$ triggers PI3K inactivation. One hypothesis is that SHP-2 binding to $\mathrm{SST}_{2}$, induced by SRIF through ITIM phosphorylation on $\mathrm{SST}_{2}$ third ICL and C-terminal domain (Ferjoux et al., 2003), triggers dephosphorylation of FLNA and p85 binding sites in $\mathrm{SST}_{2}$ first intracellular domain. Alternatively, the scaffolding FLNA protein, 
once recruited onto $\mathrm{SST}_{2}$ in the presence of SRIF (Najib et al., 2012), brings $\mathrm{SST}_{2}$ in proximity to the phosphatase SHP-1, whose activity is critical to trigger SRIF-inhibitory effects on cell proliferation, migration, or invasion. Both rat and human $\mathrm{SST}_{2}$ form constitutive homodimers (Pfeiffer et al., 2001; Lehmann et al., 2016). The dynamics of ligand-induced trafficking have also been studied for pig $\mathrm{SST}_{2}$ (Durán-Prado et al., 2007). This receptor forms constitutive homodimers/multimers in the absence of ligand, which rapidly dissociate (11 seconds) upon SRIF binding. Interestingly, in contrast to human $\mathrm{SST}_{2}$, pig $\mathrm{SST}_{2}$ rapidly reassociates (110.5 seconds) during a subsequent process that temporally overlaps with receptor internalization (half-maximal 95.1 seconds) (DuránPrado et al., 2007). When coexpressed heterologously, $\mathrm{SST}_{2}$ and $\mathrm{SST}_{3}$ form heterodimers with reduced $\mathrm{SST}_{3}$ activity (Pfeiffer et al., 2001). However, to what extent $\mathrm{SST}_{2}$ forms dimers or oligomers with other GPCRs in vivo is not known.

\section{F. Somatostatin Receptor 2 Anatomic Framework}

1. Central and Peripheral Nervous System. Two independent laboratories using two different antibodies directed toward the carboxy-terminal tail of the $\mathrm{SST}_{2}$ have demonstrated that this SST subtype is the most abundant SST in the rodent CNS (Schindler et al., 1997; Dournaud et al., 1996) in agreement with both in situ hybridization experiments and autoradiographical studies using $\mathrm{SST}_{2}$-preferring ligands. Strong $\mathrm{SST}_{2}$ labeling is detected in the deep layers of the cerebral cortex, CA1 field, and dentate gyrus of the hippocampus, lateral septum, medial septum/diagonal band of Broca, medial habenula, bed nucleus of the stria terminalis, endopiriform nucleus, claustrum, amygdaloid complex, locus coeruleus, and nucleus tractus solitarius. In the hypothalamus, the highest densitiy of $\mathrm{SST}_{2}$ immunoreactivivity is located in the ARC and the medial tuberal nucleus as well as in the lateroanterior nucleus and the ventrocaudal part of the tuber cinereum (Csaba et al., 2003). In the rat spinal cord, $\mathrm{SST}_{2}$ neurons are localized in the superficial layers of the dorsal horn (Schindler et al., 1997, 1998a; Schulz et al., 1998b,c; Segond von Banchet et al., 1999) often closely apposed by SRIFimmunoreactive terminals (Schulz et al., 1998b). In the rat retina, several neuronal cell types express $\mathrm{SST}_{2}$. In the outer layers, immunoreactivity is localized to cone photoreceptors, horizontal cells, and rod and cone bipolar cells. In the inner layers, $\mathrm{SST}_{2}$ immunostaining is present in numerous medium- to large-size amacrine cells (Johnson et al., 1999). Regional distribution of $\mathrm{SST}_{2}$ immunostaining in the human CNS is generally congruent with that reported for the rat, although, in contrast to rodents, human cerebellum displayed significant $\mathrm{SST}_{2}$ immunostaining (Schindler et al., 1998b; Csaba et al., 2005; Shi et al., 2014).

In the rat peripheral nervous system, medium-size neurons distinct from those expressing SRIF display
$\mathrm{SST}_{2}$ in the DRG (Schulz et al., 1998b). In the human and rat GIT, $\mathrm{SST}_{2}$ are localized in neurons of the myenteric and submucosal plexuses, and in fibers distributed to the muscle, mucosa, and vasculature (Sternini et al., 1997; Reubi et al., 1999).

Immunohistochemical experiments have examined localization of $\mathrm{SST}_{2 \mathrm{~B}}$ (Schulz et al., 1998a; Schindler et al., 1999). In the rat brain, somatodendritic labeling is evident in several regions that also exhibit $\mathrm{SST}_{2}$ immunostaining, including the olfactory bulb, cerebral cortex, hippocampal formation, septal nuclei, and superior colliculi. In contrast to $\mathrm{SST}_{2}$, the Purkinje cell layer of the cerebellum appears to be $\mathrm{SST}_{2 \mathrm{~B}}$ immunoreactive. In the rat spinal cord, whereas the $\mathrm{SST}_{2}$ is confined to the superficial layers, $\mathrm{SST}_{2 \mathrm{~B}}$ is located in neuronal perikarya and proximal dendrites throughout the gray matter of the spinal cord (Schulz et al., 1998a).

2. Pituitary. Using specific antibodies against $\mathrm{SST}_{2}$, it appears that this receptor is largely distributed in the adult rat and human pituitary (Fig. 9) (Mezey et al., 1998; Peineau et al., 2014). Although all anterior pituitary cell types express the $\mathrm{SST}_{2}$ protein, $\mathrm{GH}$-expressing cells almost completely colocalize with the $\mathrm{SST}_{2}$, whereas $50 \%$ of gonadotrophs, $60 \%$ of corticotrophs, $30 \%$ of thyrotrophs, and $10 \%$ of prolactin cells exhibit $\mathrm{SST}_{2}$ immunoreactivity (Peineau et al., 2014). Of note, some discrepancies with distribution of the mRNA exist because the $\mathrm{SST}_{2}$ mRNA was previously found in $40 \%$ of somatotrophs, $36 \%$ of thyrotrophs, $26 \%$ of lactotrophs, $3 \%$ of corticotrophs, and $8 \%$ of gonadotrophs (Day et al., 1995).

3. Peripheral Organs. Immunohistochemical studies also revealed the presence of the $\mathrm{SST}_{2}$ in striated ducts of the parotid gland, in neuroendocrine and enterochromaffin-like cells of the GI mucosa, in enteric ganglia, in insulin- and glucagon-secreting cells of the pancreas (Fig. 9), in the reticular zone of the adrenal cortex, in glomeruli and tubules of the kidney, in luteinized granulosa cells of the ovary, in basal parts of testicular tubules, in granulocytopoietic cells of the bone marrow, in alveolar macrophages of the lung, and in germinal centers of lymph follicles (Fischer et al., 2008; Lupp et al., 2011; Unger et al., 2012; Stollberg et al., 2016). All in all, $\mathrm{SST}_{2}$ was the most frequently detected SST subtype, and, in most cases, $\mathrm{SST}_{2}$ predominantly immune-stained at the cell plasma membrane.

4. Tumors. As determined using the rabbit $\mathrm{mAb}$ UMB-1, $\mathrm{SST}_{2}$ also represents the most prominent SST subtype in tumor tissues, with only few exceptions, such as pituitary adenomas (Fischer et al., 2008). In somatotroph and thyrotroph pituitary adenomas, $\mathrm{SST}_{2}$ is present at high abundance in $>80 \%$ of tumor specimens, along with a high presence of $\mathrm{SST}_{5}$. In contrast, in gonadotroph, corticotroph, and nonfunctioning pituitary adenomas where $\mathrm{SST}_{2}$ expression was low or even absent, a preponderance of $\mathrm{SST}_{3}$ or $\mathrm{SST}_{5}$ has been noted (Fischer et al., 2008; Lupp et al., 2011). In brain tumors, 

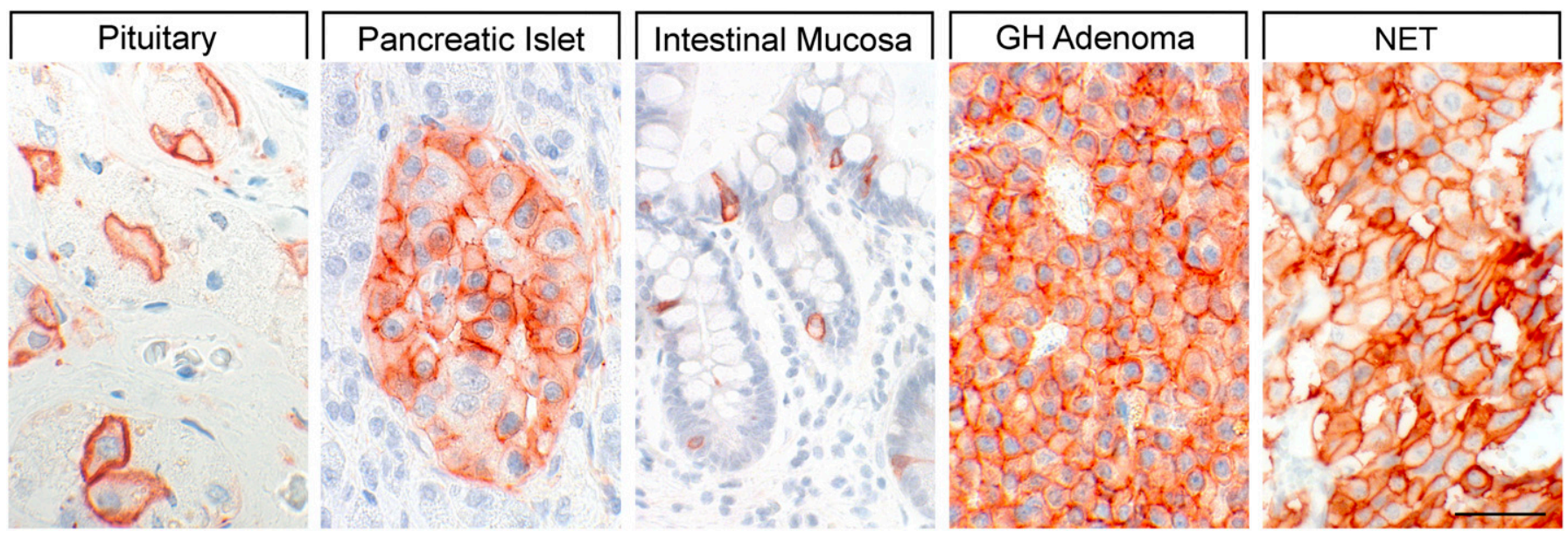

Fig. 9. $\mathrm{SST}_{2}$ expression pattern in normal human and neoplastic tissues. Immunohistochemistry (red-brown color), counterstaining with hematoxylin; primary antibody: UMB-1; scale bar, $50 \mu \mathrm{m}$. Note that $\mathrm{SST}_{2}$ is predominantly expressed at the plasma membrane.

the prevalence of $\mathrm{SST}_{2}$ was highest. $\mathrm{SST}_{2}$ was present in most of meningiomas (Schulz et al., 2000b; Fischer et al., 2008), medulloblastomas (Guyotat et al., 2001; Cervera et al., 2002; Remke et al., 2013), neuroblastomas (Albers et al., 2000), and supratentorial primitive neuroectodermal tumors of childhood (Fruhwald et al., 2004). $\mathrm{SST}_{2}$ was also detected in oligodendrogliomas, but much less frequently in astrocytomas (Cervera et al., 2002; Kiviniemi et al., 2017). Furthermore, noticeable $\mathrm{SST}_{2}$ expression occurred in peripheral nerve sheath tumors, especially in schwannomas (Mawrin et al., 2005).

$\mathrm{SST}_{2}$ was present in $43 \%-66 \%$ of medullary as well as in papillary and follicular thyroid carcinomas (Papotti et al., 2001; Druckenthaner et al., 2007; Mussig et al., 2012; Pazaitou-Panayiotou et al., 2012; Atkinson et al., 2013; Woelfl et al., 2014; Herac et al., 2016). $\mathrm{SST}_{2}$ was also detected in a high percentage $(>70 \%)$ of pheochromocytomas and paragangliomas (Fischer et al., 2008; Lupp et al., 2011; Saveanu et al., 2011; Elston et al., 2015) as well as in 33\% of both functioning and nonfunctioning adrenocortical adenomas (Unger et al., 2008). Very low levels of $\mathrm{SST}_{2}$ were also observed in lymphomas (Dalm et al., 2004; Stollberg et al., 2016; Ruuska et al., 2018). $\mathrm{SST}_{2}$ represents by far the most prominent SST subtype detected in gastroenteropancreatic (GEP)-NETs, and, overall, it was identified in $>70 \%$ of cases at a high expression intensity (Kulaksiz et al., 2002; Fischer et al., 2008; Corleto et al., 2009; Srirajaskanthan et al., 2009; Zamora et al., 2010; Lupp et al., 2011; Okuwaki et al., 2013; Kaemmerer et al., 2015b; Mehta et al., 2015; Qian et al., 2016; Wang et al., 2017). However, $\mathrm{SST}_{2}$ was detected more frequently in gastrinomas $(100 \%)$ and in carcinoid tumors $(86 \%)$ (Fig. 9) than in insulinomas (58\%) (Kulaksiz et al., 2002). $\mathrm{SST}_{2}$ expression was also found to be higher in functioning than in nonfunctioning tumors (Zamora et al., 2010; Song et al., 2016) and more pronounced in gastroenteric than in pancreatic neoplasms (Zamora et al., 2010). Negative correlation between $\mathrm{SST}_{2}$ expression and tumor grading or proliferation rate and a positive association with patient outcomes have been shown (Corleto et al., 2009; Srirajaskanthan et al., 2009; Zamora et al., 2010; Okuwaki et al., 2013; Kaemmerer et al., 2015b; Mehta et al., 2015; Qian et al., 2016; Song et al., 2016; Wang et al., 2017). Furthermore, a positive correlation between $\mathrm{SST}_{2}$ expression and SST-based imaging was demonstrated (Srirajaskanthan et al., 2009; Diakatou et al., 2015; Kaemmerer et al., 2015b). $\mathrm{SST}_{2}$ was detected in $32 \%-56 \%$ of bronchopulmonary NETs. However, in comparison with GEP-NETs, $\mathrm{SST}_{2}$ expression was less pronounced. Lower $\mathrm{SST}_{2}$ expression in high-grade in comparison with low-grade tumors and a positive correlation with SST-based imaging were shown (Righi et al., 2010; Kaemmerer et al., 2015a; Lapa et al., 2016). $\mathrm{SST}_{2}$ expression was also observed in NETs of other origins, including thymus, breast, cervix, or prostate (Kajiwara et al., 2009; Mizutani et al., 2012). Furthermore, $\mathrm{SST}_{2}$ was detected in $88 \%-100 \%$ of GI stromal tumors (GIST), and, also in this tumor entity, an association with favorable patient outcomes was demonstrated (Palmieri et al., 2007; Arne et al., 2013; Zhao et al., 2014). Depending on tumor grade and location, $\mathrm{SST}_{2}$ was observed in $45 \%-100 \%$ of colorectal carcinomas (Qiu et al., 2006; Evangelou et al., 2012) and in 41\%$67 \%$ of HCCs (Blaker et al., 2004; Reynaert et al., 2004; Verhoef et al., 2008); $\mathrm{SST}_{2}$ was expressed in 20\%-79\% of breast cancers (Pilichowska et al., 2000; Orlando et al., 2004; Kumar et al., 2005; Fischer et al., 2008; Lupp et al., 2011; Frati et al., 2014), in 57\% of cervical carcinomas, in $39 \%$ of endometrial cancers (Schulz et al., 2003), and in $30 \%$ of ovarian carcinomas (Hall et al., 2002; Schulz et al., 2003). Furthermore, a moderate to strong $\mathrm{SST}_{2}$ expression was observed in $13 \%$ of prostate cancers in general and in 50\% of prostate cancers with endocrine differentiation (Matei et al., 2012; Hennigs et al., 2014). $\mathrm{SST}_{2}$ was detected in $59 \%$ of Merkel cell carcinomas (Gardair et al., 2015) and in melanomas 
(Ardjomand et al., 2003; Valsecchi et al., 2013). Finally, $\mathrm{SST}_{2}$ expression in normal exocrine pancreatic tissue is progressively lost during pancreatic ductal adenocarcinoma progression (Buscail et al., 1996; Laklai et al., 2009), which participates in tumor aggression, as demonstrated in mouse models of pancreatic cancer combined with $\mathrm{SST}_{2} \mathrm{KO}$ mice (Chalabi-Dchar et al., 2015). Accordingly, in vitro and in vivo re-expression of $\mathrm{SST}_{2}$ in human pancreatic cancer cell lines through $\mathrm{SST}_{2} \mathrm{cDNA}$ transfection (Delesque et al., 1997; Guillermet et al., 2003; Laval et al., 2014) and through in vivo $\mathrm{SST}_{2}$ gene transfer in mouse models (Vernejoul et al., 2002) and in a first-in-man phase I clinical trial (Buscail et al., 2015), respectively, demonstrated promising oncosuppressive activity in advanced pancreatic cancer.

\section{G. Somatostatin Receptor 2 Function}

1. Endocrine System. SRIF was originally described as an inhibitor of $\mathrm{GH}$ release, but it also inhibits secretion of other pituitary hormones. $\mathrm{SST}_{2}$ is predominantly responsible for regulation of physiologic secretion of GH and TSH (Ben-Shlomo and Melmed, 2010) indirectly mediated by opening of $\mathrm{K}^{+}$channels. The subsequent $\mathrm{K}^{+}$-derived membrane hyperpolarization and reduction of $\mathrm{L}$ - and $\mathrm{N}$-type $\mathrm{Ca}^{2+}$ influx as well as intracellular $\mathrm{Ca}^{2+}$ concentration are major mechanisms by which SRIF, through the $\mathrm{SST}_{2}$ subtype, acutely inhibits exocytosis of hormone-containing vesicles (Ben-Shlomo and Melmed, 2010). $\mathrm{SST}_{2}$ also inhibits exocytosis of hormone-containing vesicles derived from pancreatic $\alpha$ - and $\beta$-cells. In rodents secretion of glucagon and in humans secretion of both glucagon and insulin are regulated by $\mathrm{SST}_{2}$ (Singh et al., 2007; Strowski and Blake, 2008; Kailey et al., 2012). In addition, the $\mathrm{SST}_{2}$ subtype activates $\mathrm{K}_{\mathrm{ir}} 3 . \mathrm{x}$, which leads to hyperpolarization and inhibits voltage-gated P/Q-type $\mathrm{Ca}^{2+}$ channels (Kailey et al., 2012).

2. Central Nervous System.

a. Neuronal excitability and epilepsy. Another major role of $\mathrm{SST}_{2}$ is inhibitory neuromodulation. Effects of $\mathrm{SST}_{2}$ on neuronal excitability have been studied in several CNS cell populations. Activation of $\mathrm{SST}_{2}$ in medial septal GABAergic neurons results in decreased discharge rate and consequent reduction of hippocampal $\theta$ rhythm power (Bassant et al., 2005). $\mathrm{SST}_{2}$ also mediates hyperpolarization of dorsal horn neurons and subsequent antinociceptive effects (Song et al., 2002; Yin et al., 2009; Shi et al., 2014). In the ventrolateral medulla, $\mathrm{SST}_{2}$ activation of presympathetic neurons provokes robust sympathoinhibition with bradycardia and hypotension (Burke et al., 2008). Rodent and sheep gonadotropin-releasing hormone neurons are inhibited by $\mathrm{SST}_{2}$, which results in decreased luteinizing hormone secretion (Bhattarai et al., 2010; McCosh et al., 2017). Activation of $\mathrm{SST}_{2}$ in olfactory bulb mitral cells modulates dendrodendritic inhibition between mitral and granule cells, which in turn results in increased $\gamma$ oscillation power of mitral cells and increased odor discrimination performances (Lepousez et al., 2010). The role of SRIF in neuronal excitability has been mostly studied in the hippocampal formation. In CA1 pyramidal neurons, SRIF has hyperpolarizing effects through activation of $\mathrm{K}^{+}$channels (Moore et al., 1988; Schweitzer et al., 1990, 1998; Tallent and Siggins, 1997).

Postsynaptic hyperpolarization of CA3 pyramidal neurons by SRIF has also been demonstrated (Tallent and Siggins, 1999). Presynaptic inhibition of glutamate release by SRIF reduces excitatory synaptic input on CA1 neurons (Kozhemyakin et al., 2013); thus, SRIF decreases both post- and presynaptic hippocampal pyramidal cell excitability (Tallent and Qiu, 2008). By contrast, in the dentate gyrus, SRIF has no effect on granule cell postsynaptic currents or firing properties. SRIF, however, inhibits postsynaptic $\mathrm{N}$-type $\mathrm{Ca}^{2+}$ channels in granule cells, resulting in inhibition of long-term potentiation (Baratta et al., 2002), a form of synaptic plasticity, critical in learning and memory, and also plays an important role in epileptogenesis. Converging evidence suggests that, of the five $\mathrm{SST}_{\mathrm{s}}, \mathrm{SST}_{2}$ exerts a predominant role in transduction of SRIF actions in the hippocampal formation (Csaba et al., 2004, 2005). SRIF plays a prominent role in epilepsy in agreement with its inhibitory neuromodulatory function, and $\mathrm{SST}_{2}$ mediates most of antiepileptic actions of SRIF in rats (Vezzani and Hoyer, 1999; Binaschi et al., 2003; Baraban and Tallent, 2004; Cervia and Bagnoli, 2007; Tallent and Qiu, 2008; Viollet et al., 2008), likely in humans (Csaba et al., 2005), but not in mice (Moneta et al., 2002).

b. Motor control. Striatal $\mathrm{SST}_{2}$ receptors are involved in control of extrapyramidal motor systems, as activation of $\mathrm{SST}_{2}$ receptors in rats increases locomotor activity (Marazioti et al., 2008; Santis et al., 2009), whereas disruption of the $\mathrm{SST}_{2}$ receptor gene in mice impairs motor functions (Viollet et al., 2000; Allen et al., 2003).

c. Feeding and drinking. In accordance with the widespread distribution of $\mathrm{SST}_{2}$ in the hypothalamus, the receptor also plays a role in drinking and feeding behavior. Activation of $\mathrm{SST}_{2}$ increases food intake by suppressing satiety (i.e., a mechanism delaying onset of another meal after a completed one), but not satiation (a mechanism causing meal termination) (Stengel et al., 2015). Increased meal numbers mediated by $\mathrm{SST}_{2}$ activation likely involve lateral hypothalamic orexinergic-A neurons projecting to the arcuate neuropeptide Y neurons that express orexin receptors $1 . \mathrm{SST}_{2}$ actions on orexinergic neurons, however, seem to be indirect (Stengel et al., 2015). Activation of $\mathrm{SST}_{2}$ also increases rapid-onset water consumption (Karasawa et al., 2014). This dipsogenic function involves activation of the angiotensin II receptor type 1 signaling system. SRIF release in the hypothalamus follows a circadian rhythm, with the highest release at the beginning of the dark 
phase in rats. Early nocturnal drinking and feeding in rats are therefore physiologically regulated by $\mathrm{SST}_{2}$ signaling (Stengel et al., 2015).

d. Stress response. Stress responses are inhibited by $\mathrm{SRIF}$, and $\mathrm{SST}_{2}$ plays a major role in inhibition of acute stress induced at several levels. First, stress-related endocrine responses are inhibited by $\mathrm{SST}_{2}$ at both the CNS and pituitary levels (Prevot et al., 2017; Stengel and Taché, 2017). Second, stress-related sympathetic activation is inhibited by $\mathrm{SST}_{2}$ at the level of brainstem presympathetic neurons (Burke et al., 2008). Finally, behavioral stress responses, such as suppression of food intake and anxiety, are also inhibited by $\mathrm{SST}_{2}$ (Stengel et al., 2015; Stengel and Taché, 2017). In addition to anxiolytic effects, $\mathrm{SST}_{2}$ also mediates antidepressant actions of SRIF (Engin et al., 2008; Engin and Treit, 2009; Fee et al., 2017; Prevot et al., 2017).

3. Retina. In the retina, $\mathrm{SST}_{2}$ inhibits adenylyl cyclase, $\mathrm{K}^{+} / \mathrm{Ca}^{2+}$ conductances, as well as activates guanylyl cyclase and NOS, and plays an important role in positive control of dopamine and negative control of glutamate release (Cervia et al., 2008). $\mathrm{SST}_{2}$ signaling through these diverse intracellular pathways converges into an important retinal neuroprotection (Casini et al., 2005; Vasilaki and Thermos, 2009). Therefore, SRIF administration is a promising therapeutic approach in treating retinal diseases involving ischemia and excitotoxicity, and a multicentric, phase II-III, randomized, controlled clinical trial (EUROCONDOR-278040) is underway to assess the efficacy of SRIF administration in diabetic retinopathy (Hernandez and Simo, 2013; Hernandez et al., 2014; Simo and Hernandez, 2014).

\section{H. Somatostatin Receptor 2 Ligands}

Early efforts to develop stable SRIF analogs with potent inhibitory activity on GH release have led to the synthesis of $\mathrm{SST}_{2}$-preferring peptide ligands, two of which, octreotide and lanreotide, have later been approved for clinical use. Both exhibit potent (subnanomolar) full agonistic properties at the $\mathrm{SST}_{2}$ and modest activity (low nanomolar) at $\mathrm{SST}_{5}$. In clinical practice, however, octreotide and lanreotide fully normalize $\mathrm{GH}$ and IGF-1 in only $\sim 50 \%$ of unselected acromegalic patients (Carmichael et al., 2014), which has stimulated the search for new SRIF analogs. This has led to recent development of pasireotide, which exhibits affinity for multiple SSTs. Pasireotide is particularly potent at the $\mathrm{SST}_{5}$, but binds with modest affinity to $\mathrm{SST}_{2}$, where it exhibits only partial agonistic activity (Bruns et al., 2002; Lesche et al., 2009; Poll et al., 2010). BIM-23120 is a highly selective $\mathrm{SST}_{2}$ peptide agonist often used to study $\mathrm{SST}_{2}$ activity (Gruszka et al., 2012; Günther et al., 2016). L-779,976 was the first selective nonpeptide agonist (Fig. 10; Table 5) (Rohrer et al., 1998) with high $\mathrm{SST}_{2}$ selectivity. However, further development of L-779,976 was halted because of low oral bioavailability. Abundant $\mathrm{SST}_{2}$ expression in human tumors has stimulated a continued search for orally available $\mathrm{SST}_{2}$-selective agonists such as L-054,264, RFE-007, as well as novel $\beta$-methyltryptophan derivatives (Yang et al., 1998; Palii et al., 2008; Banno et al., 2017). Several peptide antagonists of $\mathrm{SST}_{2}$ have also been identified, such as BIM23627 and BIM-23454. At high concentrations these compounds exhibit some residual agonist activity and are thus weak partial agonists (Pöll et al., 2010). Among available $\mathrm{SST}_{2}$ peptide antagonists, JR11 is currently the most potent and selective one (Fig. 10; Table 5) (Cescato et al., 2008).

\section{Somatostatin Receptor 3}

\section{A. Somatostatin Receptor 3 Structure}

The mouse $\mathrm{SST}_{3}$ was cloned as the third member of the SST family (Yasuda et al., 1992), and cloning of the human $\mathrm{SST}_{3}$ was reported shortly thereafter (Yamada et al., 1992b). The gene encoding for human $\mathrm{SST}_{3}$ is localized on chromosome 22q13.1 and spreads over eight exons. However, the entire coding region is localized in a single exon and encodes a protein of 418 amino acids (Fig. 11). The $\mathrm{SST}_{3}$ protein sequence shares $46 \%$ homology with $\mathrm{SST}_{2}$. Analysis of the sequence showed two potential $\mathrm{N}$-glycosylation sites located in the aminoterminal domain at $\mathrm{Asn}^{17}$ and $\mathrm{Asn}^{30}$. The genes encoding mouse and rat $\mathrm{SST}_{3}$ are localized on chromosome $15 \mathrm{E} 1$ and $7 \mathrm{q} 34$, respectively; both encode a protein of 428 amino acids. In rat $\mathrm{SST}_{3}, \mathrm{Asp}^{124}$ is essential for binding of the endogenous peptide ligand SRIF-14 (Nehring et al., 1995). A unique feature of mouse and rat $\mathrm{SST}_{3}$ is selective targeting to primary neuronal cilia (Händel et al., 1999) dependent on the presence of a conserved ciliary targeting motif within the third ICL $\left({ }^{243} \mathrm{APSCQWVQAPACQ}{ }^{255}\right)$. This sequence is identical in mouse and rat and contains a dual (AX[A/S $] \mathrm{XQ})$ ciliary targeting motif found in GPCRs efficiently localized to cilia (Berbari et al., 2008a; Jin et al., 2010; Geneva et al., 2017). In contrast, the third ICL of the human $\mathrm{SST}_{3}$ contains only a single (AX[A/S]XQ) motif. Consequently, the human $\mathrm{SST}_{3}$ is not selectively localized to primary cilia but is predominantly observed at the plasma membrane in many cell types. The calculated mol. wt. of the nonglycosylated protein is approximately $46 \mathrm{kDa}$. However, in Western blots derived from human pituitary and transfected cells, $\mathrm{SST}_{3}$ is detected as a broad smear of approximately $70-80 \mathrm{kDa}$ (Lupp et al., 2012). After peptide N-glycosidase F (PNGase F) treatment, the mol. wt. is reduced to approximately $48 \mathrm{kDa}$, and the receptor protein appears as a sharp band (Lupp et al., 2012), indicating that the native $\mathrm{SST}_{3}$ protein is indeed heavily glycosylated. $\mathrm{SST}_{3}$ is unique among SST subtypes in that it exhibits a very long carboxyl-terminal tail. In contrast to all other SSTs, the $\mathrm{SST}_{3}$ carboxyl-terminal tail lacks a potential palmitoylation site. Amino acid sequences of both the third ICL and the carboxyl-terminal tail are not conserved across species. 


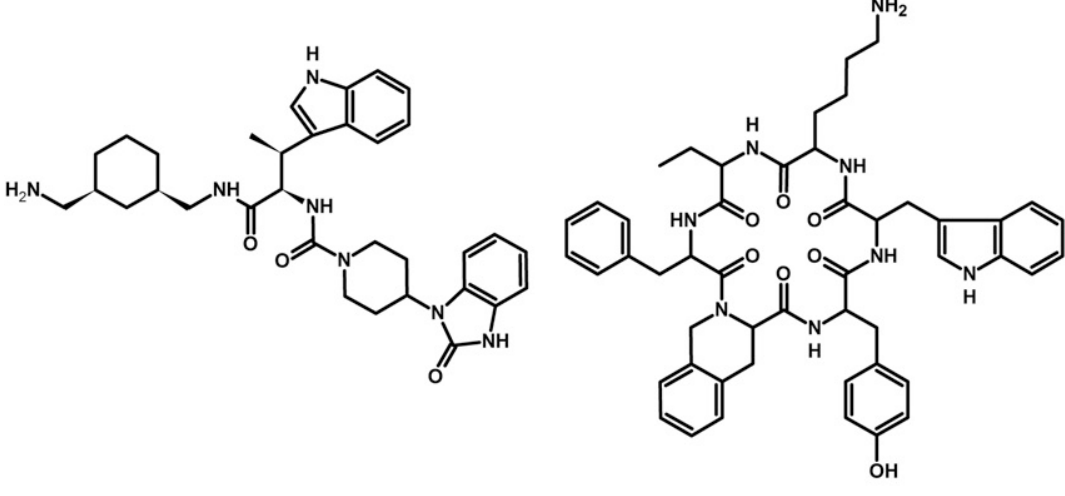

L-779,976

BIM-23120

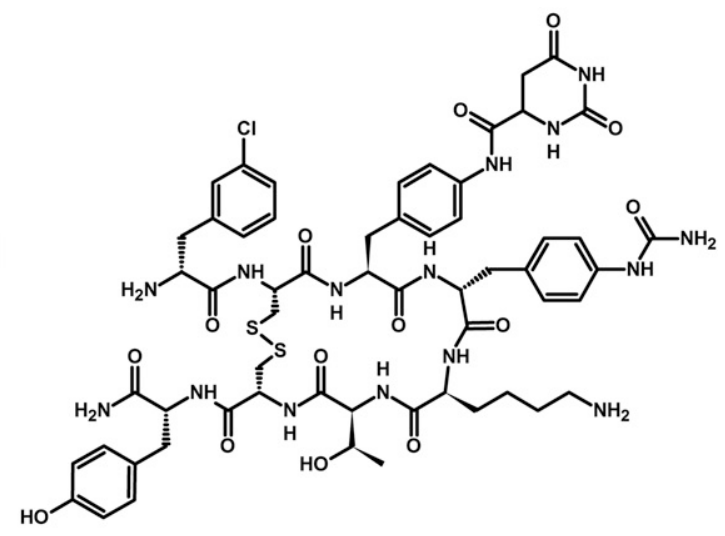

JR-11

Fig. 10. Structures of synthetic $\mathrm{SST}_{2}$ ligands. L-779,976 and BIM-23120, $\mathrm{SST}_{2}$ agonists; JR-11, $\mathrm{SST}_{2}$ antagonist.

\section{B. Somatostatin Receptor 3 Signaling Mechanisms}

$\mathrm{SST}_{3}$ is a $\mathrm{G}_{\mathrm{i} / 0}$-coupled receptor. Agonist activation results in increased incorporation of guanosine 5'-O[gamma-thio] triphosphate $(\mathrm{GTP} \gamma \mathrm{S})$ into membranes of $\mathrm{SST}_{3}$-transfected cells (Lesche et al., 2009). Its major effector systems are inhibition of adenylyl cyclase (Yamada et al., 1992b; Lesche et al., 2009), activation of $\mathrm{K}_{\mathrm{ir}} 3 . \mathrm{x}$ currents (Günther et al., 2016), and modulation of VOCCs (Fig. 12) (Mergler et al., 2008). In transfected cells, transient activation of ERK can be detected. These effects are greatly diminished when cells are preincubated with PTX, strongly indicating that $\mathrm{G}_{\mathrm{i} / \mathrm{o}}$ proteins are major effectors of $\mathrm{SST}_{3}$. Exogenously expressed human $\mathrm{SST}_{3}$ activates PLC, which is only partly blocked by PTX, suggesting involvement of $\mathrm{G}_{\mathrm{q}}$ proteins. However, the physiologic relevance of PLC activity is unknown (Siehler and Hoyer, 1999c). When expressed in stable rat pituitary tumor cells (GC cells), human $\mathrm{SST}_{3}$ exhibits constitutive ligand-independent activity that inhibits basal cAMP/protein kinase A (PKA) signaling and suppresses GH transcription through glycogen synthase kinase $3 \mathrm{~B}$ activation (Eigler et al., 2014). Heterologously expressed $\mathrm{SST}_{3}$ also induces antiproliferative or proapoptic cell-specific effects (War et al., 2015). $\mathrm{SST}_{3}$-induced apoptosis in CHO-K1 cells involves induction of transformation-related protein $53(\mathrm{p} 53)$ and Bax (Sharma et al., 1996).

\section{Somatostatin Receptor 3 Regulation}

Human $\mathrm{SST}_{3}$ contains a very long carboxyl-terminal tail of 102 amino acids, compared with only 66 and 56 residues in $\mathrm{SST}_{2}$ and $\mathrm{SST}_{5}$, respectively. Sequence homology within the carboxyl-terminal region of the mouse, rat, and human $\mathrm{SST}_{3}$ is rather low. Upon agonist exposure, human $\mathrm{SST}_{3}$ is phosphorylated at four carboxylterminal hydroxyl amino acids, namely S337, T341, T348, and S361 (Lehmann et al., 2016). Direct evidence for agonist-induced phosphorylation of these residues has been provided by generation of phosphosite-specific antibodies. For rat $\mathrm{SST}_{3}$, S341, S346, S351, and T357 have been identified as major phosphoacceptor sites using whole-cell phosphorylation assays (Roth et al., 1997a). Interestingly, phosphorylation sites for the human and rat $\mathrm{SST}_{3}$ identified to date reside within the proximal part of the $\mathrm{SST}_{3}$ carboxyl-terminal tail. Alignment of phosphorylation motifs identified in $\mathrm{SST}_{2}$ and $\mathrm{SST}_{5}$ suggests that agonist-mediated phosphorylation of these receptors occurs preferentially at a defined distance from the NPXXY motif, which marks the end of the seventh transmembrane region and the beginning of the carboxyl-terminal tail. This observation is supported by functional analysis of receptor mutants. Exchange of S/T sites to A within the proximal part of the carboxylterminal tail greatly diminished $\beta$-arrestin recruitment and $\mathrm{SST}_{3}$ internalization (Lehmann et al., 2016). In contrast, mutation of $\mathrm{S} / \mathrm{T}$ residues within the distal part of the carboxyl-terminal tail had no effect on receptor trafficking. SRIF-induced phosphorylation is completely blocked by the $\mathrm{SST}_{3}$-selective antagonist NVP-ACQ090 (Roth et al., 1997a; Lehmann et al., 2016). Phosphorylation occurs rapidly within seconds to minutes, whereas $\mathrm{SST}_{3}$ dephosphorylation occurs more slowly. In transfected HEK293 cells, agonist-induced phosphorylation is primarily mediated by $\mathrm{GRK}_{2}$ and $\mathrm{GRK}_{3}$ (Lehmann et al., 2016). The four identified phosphorylation sites do not undergo protein kinase C- or PKA-mediated phosphorylation. Dephosphorylation of $\mathrm{SST}_{3}$ specifically requires PP1 $\alpha$ and PP1 $\beta$ (Lehmann et al., 2016).

Another unique feature of the $\mathrm{SST}_{3}$ is its rapid downregulation upon prolonged agonist exposure. This effect

TABLE 5

Ligand-binding affinities of $\mathrm{SST}_{2}$-selective ligands

\begin{tabular}{lccccc}
\hline & $\mathrm{SST}_{1}$ & $\mathrm{SST}_{2}$ & $\mathrm{SST}_{3}$ & $\mathrm{SST}_{4}$ & $\mathrm{SST}_{5}$ \\
\hline L-779/976 $^{a}$ & 2760 & 0.05 & 729 & 310 & 4260 \\
BIM-23120 $^{b}$ & $>1000$ & 0.34 & 412 & $>1000$ & 213.5 \\
DOTA-JR11 $^{c}$ & $>1000$ & 0.58 & $>1000$ & $>1000$ & $>1000$ \\
\hline
\end{tabular}

${ }^{a}$ Data from Rohrer et al. (1998).

${ }^{b}$ Data from Gruszka et al. (2012).

${ }^{c}$ Data from Cescato et al. (2008). 


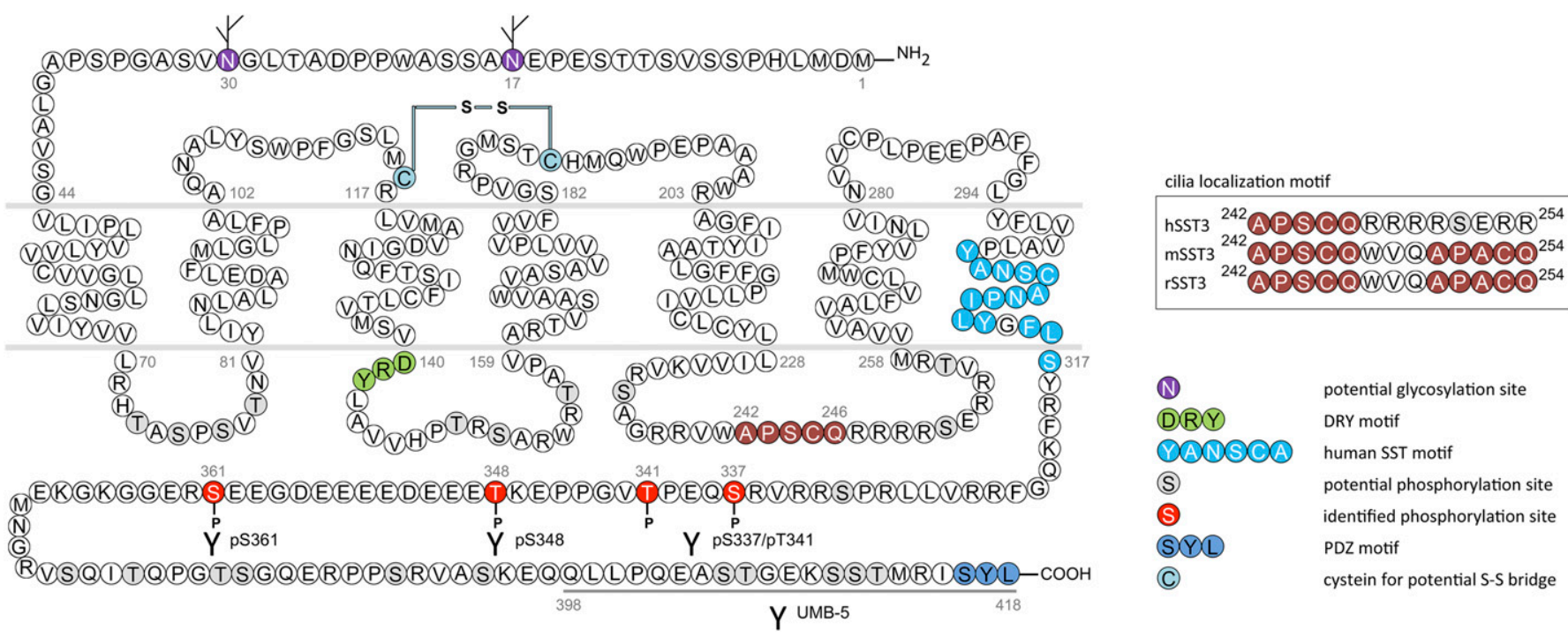

Fig. 11. Structure of human $\mathrm{SST}_{3}$. The primary and secondary amino acid structure of the human $\mathrm{SST}_{3}($ UniProtKB - P32745) is shown in a schematic serpentine format. Glycosylation sites are colored in purple; the DRY motif is highlighted in green; the human SST motif is in light blue; potential phosphorylation sites are in gray; identified GRK2/3 phosphorylation sites are in red; the PDZ ligand motif is in dark blue; the cilia localization motif is in dark red; and the disulfide-forming cysteines are in pale blue. UMB-5 is a rabbit monoclonal antibody, which detects the carboxyl-terminal tail of $\mathrm{SST}_{3}$ in a phosphorylation-independent manner.

has been clearly documented for both human and rat $\mathrm{SST}_{3}$ in transfected cells (Tulipano et al., 2004; Lesche et al., 2009; Lehmann et al., 2016). Downregulation of about $50 \%$ of cellular $\mathrm{SST}_{3}$ protein is observed between 6 and 12 hours of continued agonist exposure. Loss of human and rat $\mathrm{SST}_{3}$ occurred similarly with SRIF, octreotide, or pasireotide as ligands. In contrast, no such downregulation was observed with human $\mathrm{SST}_{2}$ or $\mathrm{SST}_{5}$ under similar conditions (Tulipano et al., 2004; Lesche et al., 2009). It is not known, however, whether endogenous $\mathrm{SST}_{3}$ undergoes such rapid downregulation. Such studies are difficult to perform because human cell lines expressing sufficient levels of endogenous $\mathrm{SST}_{3}$ allowing immunochemical detection of receptor protein are not available. Degradation of $\mathrm{SST}_{3}$ was blocked by the lysosomal inhibitor chloroquine and the cell-permeable proteasome inhibitor MG132 (Tulipano et al., 2004). For rat $\mathrm{SST}_{3}$, agonist-induced ubiquitination occurs at intracellular lysine residues. Mutation of all intracellular lysine residues to arginine in a K-R mutant of the human $\mathrm{SST}_{3}$ prevents downregulation only during the first 6 hours of treatment. After 24 hours, downregulation of the K-R mutant occurs to the same extent as for wildtype $\mathrm{SST}_{3}$, suggesting that ubiquitination of $\mathrm{SST}_{3}$ facilitates, but is not an absolute requirement for degradation (Lehmann et al., 2016).

\section{Somatostatin Receptor 3 Trafficking}

$\mathrm{SST}_{3}$ is regulated like a prototypical GPCR in that it is internalized within minutes upon exposure to the endogenous SRIF-14 ligand. The time course and extent of receptor internalization are similar to that observed for $\mathrm{SST}_{2}$. Internalization is preceded by recruitment of $\beta$-arrestins to the activated $\mathrm{SST}_{3}$ (Kreuzer et al., 2001).
In fact, $\beta$-arrestin-2 is more efficiently recruited than $\beta$-arrestin-1. Interestingly, SRIF-14 bound to internalized $\mathrm{SST}_{3}$ is rapidly degraded, whereas octreotide is recycled as an intact peptide (Roosterman et al., 2008). Phosphorylation is a precondition for arrestin translocation and internalization. $\mathrm{SST}_{3}$ and arrestin form transient complexes (Lehmann et al., 2016). Although $\mathrm{SST}_{3}$ receptors are internalized via clathrin-coated pits into early endosomes, arrestins dissociate from the receptor and redistribute into the cytosol (Tulipano et al., 2004; Lehmann et al., 2016). This is in contrast to $\mathrm{SST}_{2}$, which forms stable complexes with arrestins that cointernalize into early endosomes. After internalization, only a proportion of $\mathrm{SST}_{3}$ recycles back to the plasma membrane. Remaining intracellular receptors are transferred to larger diameter vesicles (presumably lysosomes) for degradation (Tulipano et al., 2004). This action is also in contrast to $\mathrm{SST}_{2}$, which completely recycles to the plasma membrane within 60-90 minutes after agonist removal. Differential endosomal sorting of $\mathrm{SST}_{3}$ and $\mathrm{SST}_{2}$ has been demonstrated using an immunocytochemical pulse-chase assay that estimates the degree to which internalized receptors remain associated with transferrin-containing endocytic vesicles. Transferrin is a well-established marker of early and recycling endosomes that mediate rapid recycling. After a 30-minute SRIF-14/transferrin pulse, a high degree of colocalization of both $\mathrm{SST}_{3}$ and $\mathrm{SST}_{2}$ with transferrin was observed. However, after an additional 20-minute pulse, only $\mathrm{SST}_{2}$ - but not $\mathrm{SST}_{3}$ - showed a high degree of colocalization with endocytosed transferrin, suggesting that $\mathrm{SST}_{3}$ are predominantly sorted to a population of endocytic vesicles distinct from those that constitute the conserved recycling pathway marked 


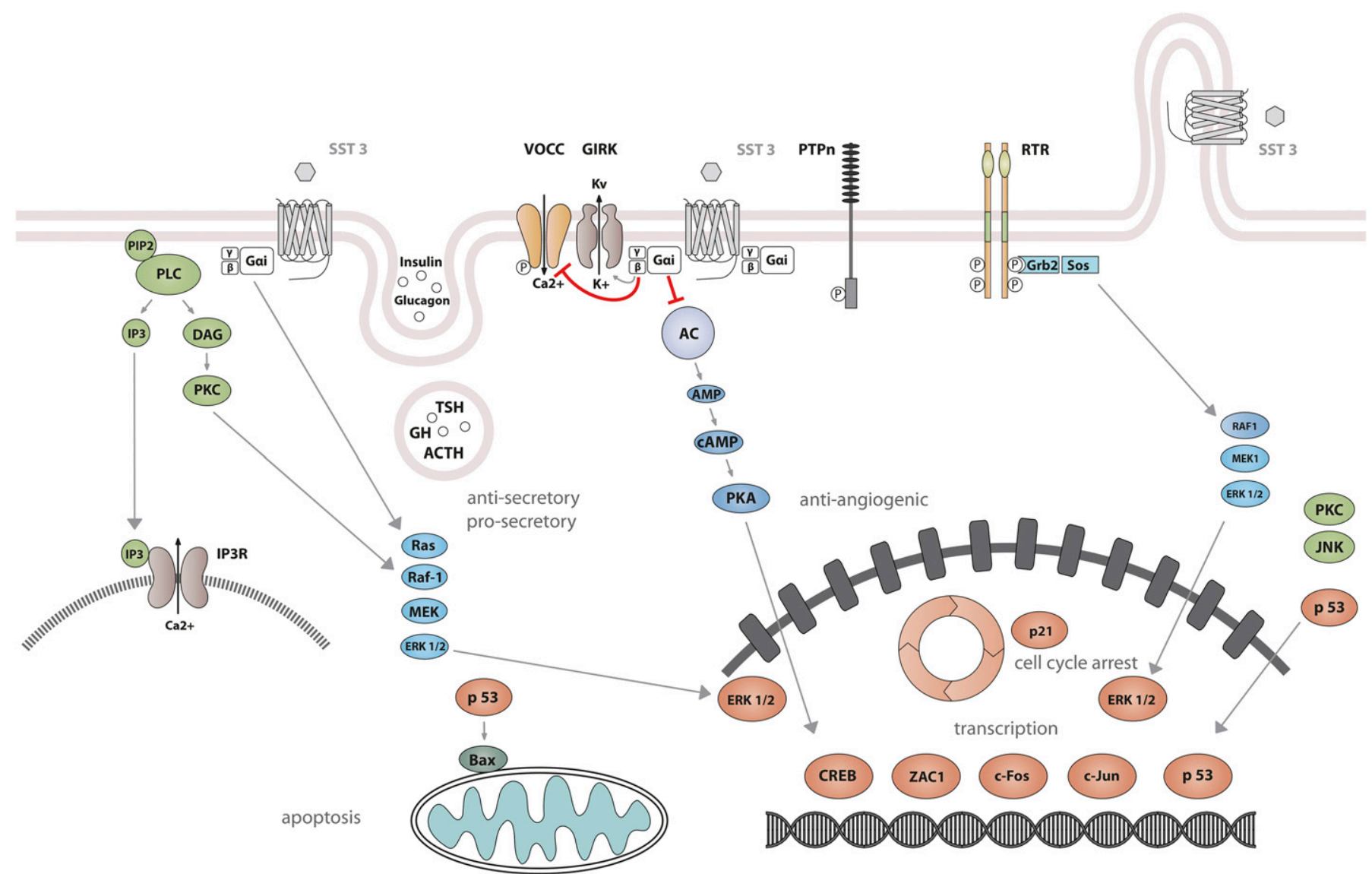

Fig. 12. $\mathrm{SST}_{3}$ signaling leading to inhibition of hormone secretion, proliferation, and induction of apoptosis. By coupling to Gi proteins, $\mathrm{SST}_{3}$ inhibits adenylate cyclase and reduces cAMP accumulation and reduces intracellular $\mathrm{Ca}^{2+}$ concentrations by activating GIRK channels, which results in membrane hyperpolarization and subsequent reduction of $\mathrm{Ca}^{2+}$ influx through VOCC. This results in decreased hormone secretion. By coupling to a pertussis toxin-independent $\mathrm{G}$ protein (probably Gq), $\mathrm{SST}_{3}$ activates PLC, triggering inositol-1,4,5-trisphosphate (IP 3 ) production and subsequent $\mathrm{Ca}^{2+}$ release into the cytoplasm from endoplasmic reticulum. $\mathrm{SST}_{3}$-dependent induction of apoptosis involves p53 and Bax.

by transferrin (Tulipano et al., 2004). Ras-related in brain (Rab) proteins are markers for specific populations of endosomes. For many GPCRs, Rab proteins are major regulators of endosomal trafficking. Using real-time imaging, it was demonstrated that $\mathrm{SST}_{3}$ traffics through Rab4-, Rab11-, and Rab21-containing endosomes. Expression of inactive variants of these specific Rab proteins inhibits passage of $\mathrm{SST}_{3}$ through different endosomal compartments (Tower-Gilchrist et al., 2011).

\section{E. Somatostatin Receptor 3 Targeting}

When expressed in HEK293 cells, mouse, rat, and human $\mathrm{SST}_{3}$ demonstrate bona fide plasma membrane localization. In contrast, $\mathrm{SST}_{1}$ is predominantly localized to intracellular vesicular compartments. In fact, transplantation of the rat $\mathrm{SST}_{3} \mathrm{~N}$-terminal domain to the rat $\mathrm{SST}_{1}$ is sufficient to facilitate plasma membrane localization of $\mathrm{SST}_{1}$, suggesting that the N-terminal domain of $\mathrm{SST}_{3}$ contains a plasma membrane targeting sequence (Ammon et al., 2002). However, when mouse $\mathrm{SST}_{3}$ is expressed exogenously in polarized innermedullary-collecting duct cells or cultured hippocampal neurons, the receptor protein is concentrated in primary cilia (Berbari et al., 2008a). Primary cilia are nonmotile plasma membrane appendages that serve specialized sensory functions, such as light sensation in photoreceptors or detection of odors in olfactory neurons. Cilia are enriched in signaling proteins, such as $G$ proteins, adenylyl cyclases, ion channels, and arrestins. Their function is defined by the presence of specific signaling receptors. Importantly, disruption of ciliary function has been associated with human ciliopathies, such as Bardet-Biedl syndrome (BBS), Joubert syndrome, and Meckel syndrome, which have prominent functional and structural CNS phenotypes (Berbari et al., 2008b; Jin et al., 2010). In fact, $\mathrm{SST}_{3}$ was the first signaling receptor identified in primary neuronal cilia in mouse and rat brain (Händel et al., 1999) and is therefore a prototypical ciliary GPCR. Thereafter, additional GPCRs with selective targeting to primary cilia were identified, such as 5-hydroxytryptamine receptor 6 and melanin-concentrating hormone receptor 1 (Ammon et al., 2002). In rodents, $\mathrm{SST}_{3}$ was also identified in primary cilia in pancreatic islets and adenohypophysis (Iwanaga et al., 2011). Expression of $\mathrm{SST}_{3}$ in neuronal cilia in rodents appears after birth when first cilia are formed and persists throughout the 
aged brain (Stanić et al., 2009; Guadiana et al., 2016). However, selective cilia targeting appears not to be conserved across many species. The dual (AX[A/S]XQ) ciliary targeting motif in the third ICL $\left({ }^{243}\right.$ APSCQWVQAPACQ $\left.{ }^{255}\right)$ is identical in rat and mouse. This motif is not found in any other SST subtype, and human $\mathrm{SST}_{3}$ contains only a single ciliary targeting motif $\left({ }^{242}\right.$ APSCQRRRRSERR $\left.{ }^{254}\right)$. This may explain why subcellular localization of the human $\mathrm{SST}_{3}$ is not restricted to primary cilia in many cell types.

\section{F. Somatostatin Receptor 3 Interacting Proteins}

The rodent $\mathrm{SST}_{3}$ is a prototypical ciliary GPCR. As such, $\mathrm{SST}_{3}$ has been intensively used to identify proteins that traffic cargo to cilia. The ciliary targeting signal of rodent $\mathrm{SST}_{3}$ is directly recognized by BBS proteins that form the BBSome complex required for targeting of membrane proteins to cilia (Jin et al., 2010). The BBSome is an octameric complex consisting of seven highly conserved BBS proteins, BBS1, BBS2, BBS4, BBS5, BBS7, BBS8, and BBS9, plus BBIP10. $\mathrm{BBS}$ is an autosomal recessive disorder characterized by retinal degeneration, polydactyly, kidney cysts, and obesity, caused by mutations in any of 14 known genes and whose etiology is associated with cilium dysfunction. In the absence of BBSome function, $\mathrm{SST}_{3}$ accumulates at the plasma membrane (Jin et al., 2010). In the naturally occurring tubby mutant mouse, which develops retinitis pigmentosa, hearing loss, and obesity, $\mathrm{SST}_{3}$ also fails to localize to cilia. Although it is not known whether the tubby protein product binds directly to $\mathrm{SST}_{3}$, it appears to be an accessory factor in ciliary GPCR trafficking (Sun et al., 2012). Once embedded into the ciliary plasma membrane, $\mathrm{SST}_{3}$ behaves as a functional GPCR. Activation by SRIF facilitates translocation of $\beta$-arrestin-2 into cilia. $\beta$-Arrestin-2 recruitment depends on $\mathrm{SST}_{3}$ phosphorylation and is required for removal of activated $\mathrm{SST}_{3}$ receptors from the ciliary space (Green et al., 2015). Interestingly, when the receptor fails to undergo BBSome- or arrestin-mediated retrieval from the cilia back into the cell, $\mathrm{SST}_{3}$ concentrates into membranous buds at the tips of cilia before release into extracellular vesicles via exocytosis (Nager et al., 2017). For the human $\mathrm{SST}_{3}$, the carboxyl-terminal domain was shown to interact with the multiple PDZdomain protein 1 (MUPP1). MUPP1 is a tight junction scaffold protein in epithelial cells, and, as a result of the interaction with MUPP1, $\mathrm{SST}_{3}$ is targeted to tight junctions in human keratinocytes. Interaction with MUPP1 enables the receptor to regulate transepithelial permeability in a PTX-sensitive manner, suggesting that human $\mathrm{SST}_{3}$ can activate $\mathrm{G}$ proteins locally at tight junctions (Liew et al., 2009). Both rat and human $\mathrm{SST}_{3}$ form constitutive homodimers (Pfeiffer et al., 2001; Lehmann et al., 2016). When coexpressed, rat $\mathrm{SST}_{2}$ and $\mathrm{SST}_{3}$ can form heterodimers with reduced $\mathrm{SST}_{3}$ activity (Pfeiffer et al., 2001).
However, to what extent $\mathrm{SST}_{3}$ can form dimers or oligomers with other GPCRs in vivo is not known.

\section{G. Somatostatin Receptor 3 Anatomic Framework}

Cellular and subcellular localizations of human $\mathrm{SST}_{3}$ have been studied in detail using rabbit mAb UMB-5, which is directed against the distal part of its carboxylterminal tail $\left({ }^{398}\right.$ QLLPQEASTGEKSSTMRISYL $\left.{ }^{418}\right)$ (Fig. 13). In normal human tissues, $\mathrm{SST}_{3}$ is present at the plasma membrane of distinct cell populations in the anterior pituitary, pancreatic islets, enteric ganglion cells of the GIT, the zona fasciculata and zona reticularis of the adrenal cortex, as well as the adrenal medulla, glomeruli and tubules of the kidney, luteinized granulosa cells of the ovary, and immune cells (Lupp et al., 2012). Interestingly, nonfunctioning pituitary adenomas (mostly of gonadotroph lineage) express high levels of $\mathrm{SST}_{3}$, whereas expression of $\mathrm{SST}_{2}$ and $\mathrm{SST}_{5}$ is very low or absent (Lupp et al., 2012; Lee et al., 2015). In most ACTHproducing adenomas, $\mathrm{SST}_{3}$ and $\mathrm{SST}_{5}$-but not $\mathrm{SST}_{2}$-are present. In contrast, rodent $\mathrm{SST}_{3}$ receptors are selectively targeted to primary cilia in pancreatic islets and anterior pituitary and to primary neuronal cilia in many brain regions, including cerebral cortex, hippocampus, hypothalamus, and amygdala (Händel et al., 1999; Iwanaga et al., 2011). Another brain region with high $\mathrm{SST}_{3} \mathrm{mRNA}$ expression is the cerebellum, where $\mathrm{SST}_{3}$ is expressed on Bergmann glial cells (Händel et al., 1999).

$\mathrm{SST}_{3}$ is also present in different human tumor types. It is consistently observed with high intensity of expression, especially in pituitary adenomas. In GH-producing pituitary adenomas, noticeable amounts of $\mathrm{SST}_{5}$ and $\mathrm{SST}_{3}$ are also expressed, besides high levels of $\mathrm{SST}_{2}$ (Lupp et al., 2012; Casar-Borota et al., 2013), whereas in most of ACTH-producing adenomas only $\mathrm{SST}_{5}$ and $\mathrm{SST}_{3}$, but not $\mathrm{SST}_{2}$, were detected (Lupp et al., 2011, 2012). In contrast, in gonadotropic and nonfunctioning pituitary tumors, high levels of $\mathrm{SST}_{3}$ were observed, whereas $\mathrm{SST}_{2}$ and $\mathrm{SST}_{5}$ expression was low or even absent (Lupp et al., 2012; Gabalec et al., 2015; Lee et al., 2015). $\mathrm{SST}_{3}$ is expressed in about $30 \%-50 \%$ of pheochromocytomas and paragangliomas (Elston et al., 2015). In addition, $\mathrm{SST}_{3}$ was found most prominently expressed in thymomas (Ferone et al., 2000). Although $\mathrm{SST}_{2}$ is clearly the most prominent receptor expressed in gastroenteropancreatic neuroendocrine neoplasms, $\mathrm{SST}_{3}$ was also detected in 52\%-90\% of cases (Lupp et al., 2012; Kaemmerer et al., 2015b; Qian et al., 2016). However, and in contrast to $\mathrm{SST}_{2}$, strong $\mathrm{SST}_{3}$ expression was only noted in 5\%-29\% of cases (Kaemmerer et al., 2015b; Qian et al., 2016; Song et al., 2016). Low $\mathrm{SST}_{3}$ expression levels occur also in bronchopulmonary neuroendocrine neoplasms (Kaemmerer et al., 2015a). Likewise, in about 50\% of tumors with neuroendocrine differentiation derived from breast, cervix, or prostate, weak to moderate $\mathrm{SST}_{3}$ expression was observed (Mizutani et al., 2012). 

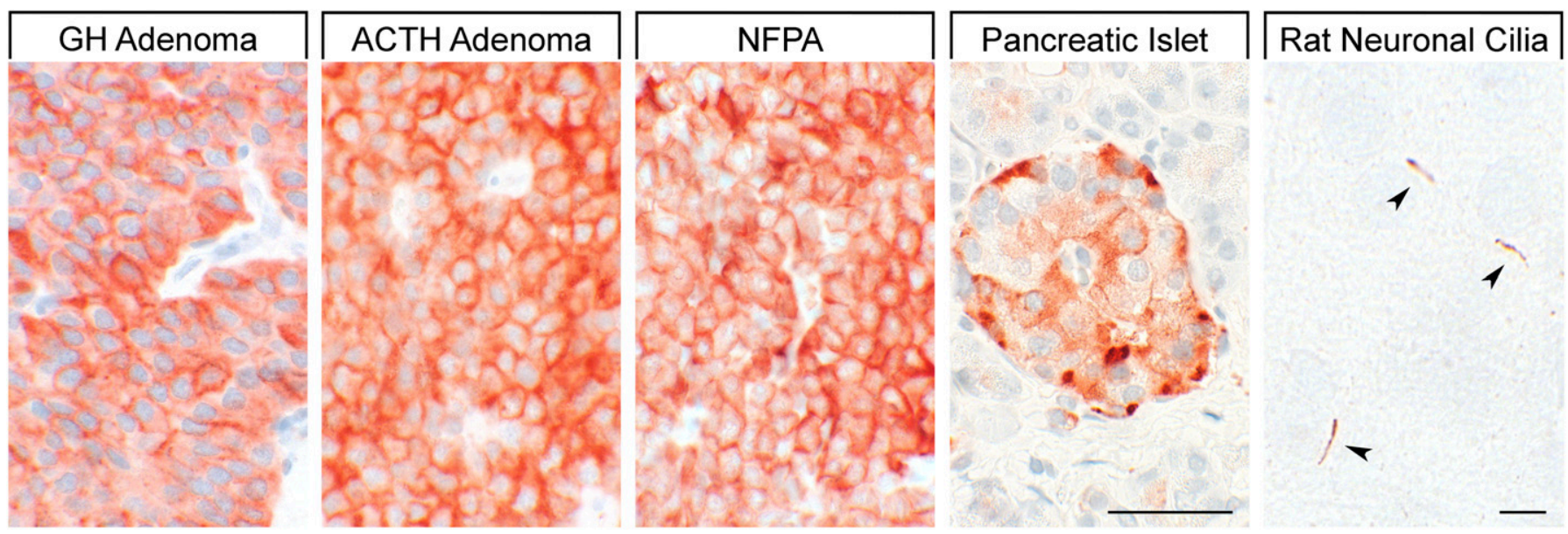

Fig. 13. $\mathrm{SST}_{3}$ expression pattern in human pituitary adenomas, human pancreatic islets, and rat neuronal cilia. Immunohistochemistry (red-brown color), counterstaining with hematoxylin; primary antibody: UMB-5; scale bar, $50 \mu \mathrm{m}$. $\mathrm{SST}_{3}$ displays both membranous and cytoplasmic expression. NFPA, clinically nonfunctioning pituitary adenoma.

$\mathrm{SST}_{3}$ was also detected in $56 \%$ of GIST tumors (Zhao et al., 2014).

\section{H. Somatostatin Receptor 3 Function}

At the cellular level, activation of $\mathrm{SST}_{3}$ inhibits hormone release. Inhibition of GH release has been observed in GC cells (Eigler et al., 2014). $\mathrm{SST}_{3}$ agonists inhibit insulin release from INS-1 cells (Mergler et al., 2008). At the systemic level, highly selective $\mathrm{SST}_{3}$ antagonists such as MK-1421 or MK-4256 facilitate glucose-stimulated insulin secretion from pancreatic $\beta$-cells and block glucose excursion in wild-type, but not $\mathrm{SST}_{3} \mathrm{KO}$ mice (Pasternak et al., 2012; Shah et al., 2015; He et al., 2016). This suggests that $\mathrm{SST}_{3}$ antagonism represents a new potential mechanism for treating type 2 diabetes mellitus. $\mathrm{SST}_{3}$ expressed in primary neuronal cilia in rodent brain is critical for object recognition memory. $\mathrm{SST}_{3} \mathrm{KO}$ mice are severely impaired in discriminating novel objects, whereas they retain normal memory for object location. Similarly, systemic injection of the $\mathrm{SST}_{3}$ antagonist NVP-ACQ090 disrupts recall of familiar objects in wild-type mice (Einstein et al., 2010). In addition, the anticonvulsant effects of CST-14 in rodents can be blocked by the selective $\mathrm{SST}_{3}$ antagonist $\mathrm{SST}_{3}$-ODN-8, suggesting that this activity is mediated in part via the $\mathrm{SST}_{3}$ (Aourz et al., 2014).

\section{Somatostatin Receptor 3 Ligands}

SRIF-14 is a full agonist at the $\mathrm{SST}_{3}$, mediating strong $G$ protein signaling, full phosphorylation, and internalization of the receptor (Fig. 14) (Lehmann et al., 2016). Compared with SRIF-14, octreotide and pasireotide behave as full agonists with regard to $G$ protein signaling and as partial agonists with regard to receptor phosphorylation and internalization (Lehmann et al., 2016). L-796,778 was the first selective nonpeptide $\mathrm{SST}_{3}$ agonist with a moderate affinity (Rohrer et al.,
1998); however, compared with SRIF-14, it behaves as a weak partial agonist on $\mathrm{G}$ protein signaling and does not induce noticeable receptor phosphorylation or internalization (Fig. 14; Table 6) (Lehmann et al., 2016). The first selective $\mathrm{SST}_{3}$ antagonist discovered, $\mathrm{SST}_{3}$-ODN8 , was successfully used to label endogenous $\mathrm{SST}_{3}$ in human tissues using autoradiographic binding studies (Fig. 14; Table 6) (Reubi et al., 2000a). Great progress has been made in development of two structurally distinct classes of selective nonpeptide $\mathrm{SST}_{3}$ antagonists based on tetrahydro- $\beta$-carboline and decahydroisoquinoline derivatives (Poitout et al., 2001; Troxler et al., 2010; He et al., 2016). The decahydroisoquinoline derivative ACQ090 is a full neutral antagonist that blocks phosphorylation and internalization of $\mathrm{SST}_{3}$ completely (Fig. 14; Table 6) (Lehmann et al., 2016). Tetrahydro- $\beta$-carboline derivatives such as MK-1421 or MK-4256 are highly selective for $\mathrm{SST}_{3}$. They were shown to facilitate glucose-stimulated insulin secretion from pancreatic $\beta$-cells and block glucose excursion in rodents in vivo (Pasternak et al., 2012; Shah et al., 2015; He et al., 2016). MK-4256 has been evaluated as a potential candidate for treatment of type 2 diabetes mellitus (Fig. 14; Table 6) (He et al., 2012, 2016; Shah et al., 2015). However, development was discontinued due to adverse cardiovascular effects related to human ether-a-go-go-related gene off-target side effects $(\mathrm{He}$ et al., 2014). Attempts to eliminate this off-target action have led to discovery of (4-phenyl-1H-imidazol-2-yl)methanamines as potent and selective $\mathrm{SST}_{3}$ agonists (Li et al., 2014; Lai et al., 2015).

\section{Somatostatin Receptor 4}

\section{A. Somatostatin Receptor 4 Structure}

cDNAs coding for rat and human $\mathrm{SST}_{4}$ were cloned in 1992 and 1993, respectively, and the rat tissue distribution of the mRNA suggested a brain-specific receptor 


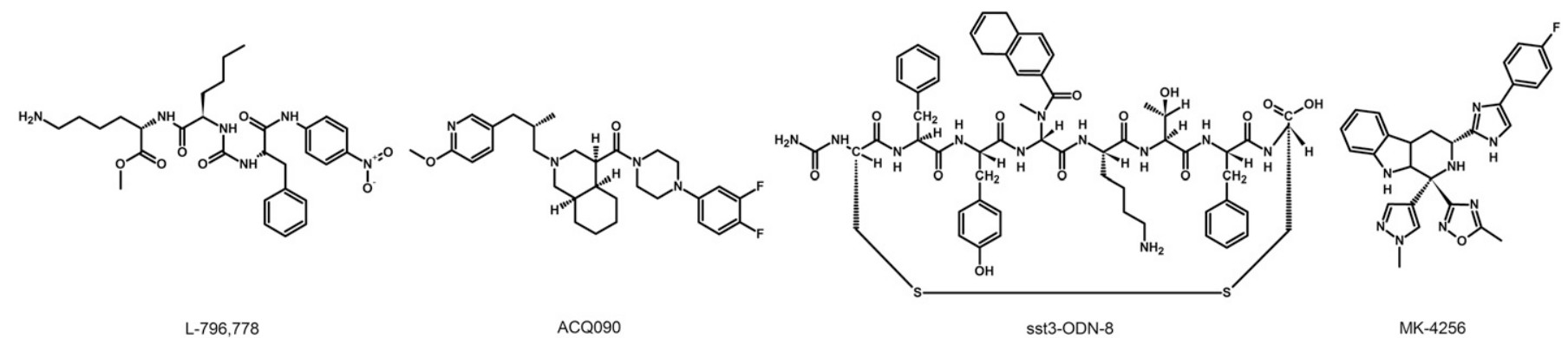

Fig. 14. Structures of synthetic $\mathrm{SST}_{3}$ ligands. L-796,776, $\mathrm{SST}_{3}$ agonist; ACQ090, sst $_{3}$-ODN-8, and MK-4256, $\mathrm{SST}_{3}$ antagonists.

subtype (Bruno et al., 1992; Demchyshyn et al., 1993; Rohrer et al., 1993). The gene encoding human $\mathrm{SST}_{4}$ is localized on chromosome 20p11.2 in a single exon. Genes encoding mouse and rat $\mathrm{SST}_{4}$ are localized on chromosomes $2 \mathrm{G} 3$ and 3q41, respectively. Human $\mathrm{SST}_{4}$ and rat $\mathrm{SST}_{4}$ are proteins of 388 and 384 amino acids, respectively, and show $88 \%$ sequence identity. Both human and rodent $\mathrm{SST}_{4}$ feature one site for N-linked glycosylation in the $\mathrm{N}$-terminal domain and a putative palmitoylation motif in the C-terminal tail. The calculated mol. wt. is approximately $42 \mathrm{kDa}$. However, in Western blots derived from rat brain, $\mathrm{SST}_{4}$ protein is detected as a broad smear of 60-70 kDa (Schreff et al., 2000). After PNGase F digestion, the mol. wt. is reduced to approximately $45 \mathrm{kDa}$ and the receptor protein appears as a sharp band (Schreff et al., 2000), indicating that the native $\mathrm{SST}_{4}$ is indeed glycosylated. $\mathrm{SST}_{4}$ shares a conserved sequence ${ }^{(351}$ YANSCANPILY $\left.{ }^{361}\right)$ (Fig. 15) in transmembrane region 7 (mammalian SST signature) and a consensus motif (X-[S/T]-X- $\Phi$ ) in its $\mathrm{C}$ terminus with all the other mammalian SST subtypes (UniProtKB accession: P31391). The X-S/T-X- $\Phi$ motif (the hydrophobic amino acid $\Phi$ is phenylalanine in $\mathrm{SST}_{4}$ ) is regarded as a potential PDZ domain binding site and might be important for interaction with scaffolding proteins (Christenn et al., 2007). Crystal structure of the agonist-bound $\mathrm{SST}_{4}$ is not yet available, and studies on ligand binding and receptor activation have been sparse. A homology model of $\mathrm{SST}_{4}$ has been generated using the active state $\beta_{2}$-adrenergic receptor crystal structure, and a number of reported ligands have been docked to the model-built structure. This molecular prediction analysis suggested two partially overlapping binding modes (Liu et al., 2012).

\section{B. Somatostatin Receptor 4 Signaling Mechanisms}

$\mathrm{SST}_{4}$ is coupled to heterotrimeric $\mathrm{G}_{\mathrm{i} / \mathrm{o}}$ protein-mediated adenylate cyclase inhibition (Fig. 16) (Demchyshyn et al., 1993; Patel et al., 1994). In cortical neurons, $\mathrm{SST}_{4}$ has been linked to activation of M currents (Qiu et al., 2008). Native $\mathrm{SST}_{4}$ modulates $\mathrm{K}_{\mathrm{ir}} 3 . \mathrm{x}$, VOCC, and transient receptor potential cation channel subfamily $\mathrm{V}$ member 1 in rat DRG neurons (Gorham et al., 2014b; Schuelert et al., 2015). In rat retinal ganglion cells, $\mathrm{SST}_{4}$ also modulates VOCC
(Farrell et al., 2010, 2014). In transfected cells, agoniststimulated $\mathrm{SST}_{4}$ activates phospholipase A2, leading to production of arachidonic acid as a second messenger, the MAPK signaling cascade (Bito et al., 1994; Smalley et al., 1999), and a NHE1 (Smalley et al., 1998). In CHO cells expressing human recombinant $\mathrm{SST}_{4}$, SRIF stimulates basal proliferation through a mechanism involving prolonged activation of mitogen-activated protein kinases 1/2 (ERK1/2) and phosphorylation of signal transducer and activator of transcription 3 (STAT3) (Sellers et al., 1999). This event is transduced by $\mathrm{G}_{\mathrm{i} / \mathrm{o}}$ proteins and is dependent on PI3K activity. However, $\mathrm{SST}_{4}$ exerts more complex functions in regulating cell proliferation. For example, $\mathrm{SST}_{4}$ causes prolonged activation of $\mathrm{p} 38$ MAPK, which in turn results in the induction of the cell cycle inhibitor p21 (cip1/Waf1) and growth arrest, when cells were simultaneously exposed to somatostatin and basic FGF (Alderton et al., 2001). When expressed in malignant pleural mesothelioma cells, human $\mathrm{SST}_{4}$ activates SHP-1 and SHP-2. Downstream signaling through SHP-2 is required for cytostatic effects of $\mathrm{SST}_{4}$ observed in these cells (Yamamoto et al., 2014). In transfected $\mathrm{CHO}$ cells, $\mathrm{SST}_{4}$ causes PI3K-dependent activation of NHE1 and increases extracellular acidification rate (Smalley et al., 1999). In contrast, $\mathrm{SST}_{4}$ inhibits the ubiquitous NHE1 in transfected rat fibroblasts (Lin et al., 2003).

\section{Somatostatin Receptor 4 Regulation}

The 5' promoter region of the human gene (Petersenn et al., 2002b) bears little homology between the human and the rat $5^{\prime}$ flanking regions (Xu et al., 1995b). A minimal -209-bp 5' flanking region contains elements that support human promoter activity in different cell

TABLE 6

Ligand-binding affinities of $\mathrm{SST}_{3}$-selective ligands

\begin{tabular}{|c|c|c|c|c|c|}
\hline & $\mathrm{SST}_{1}$ & $\mathrm{SST}_{2}$ & $\mathrm{SST}_{3}$ & $\mathrm{SST}_{4}$ & $\mathrm{SST}_{5}$ \\
\hline $\mathrm{L}-796 / 778^{a}$ & 1255 & $>10,000$ & 24 & 8650 & 1200 \\
\hline $\mathrm{sst}_{3}-\mathrm{ODN}-8^{b}$ & $>10,000$ & $>10,000$ & 4.1 & $>10,000$ & $>10,000$ \\
\hline $\mathrm{ACQ}^{2} 90^{c}$ & 5.68 & 5.31 & 8.13 & 6.81 & 5.93 \\
\hline MK- $4256^{d}$ & 2362 & 4025 & 0.66 & 384 & 533 \\
\hline
\end{tabular}

${ }^{a}$ Data from Rohrer et al. (1998).

${ }^{b}$ Data from Reubi et al. (2000a).

${ }^{c}$ Data from Troxler et al. (2010).

${ }^{d}$ Data from He et al. (2012). 


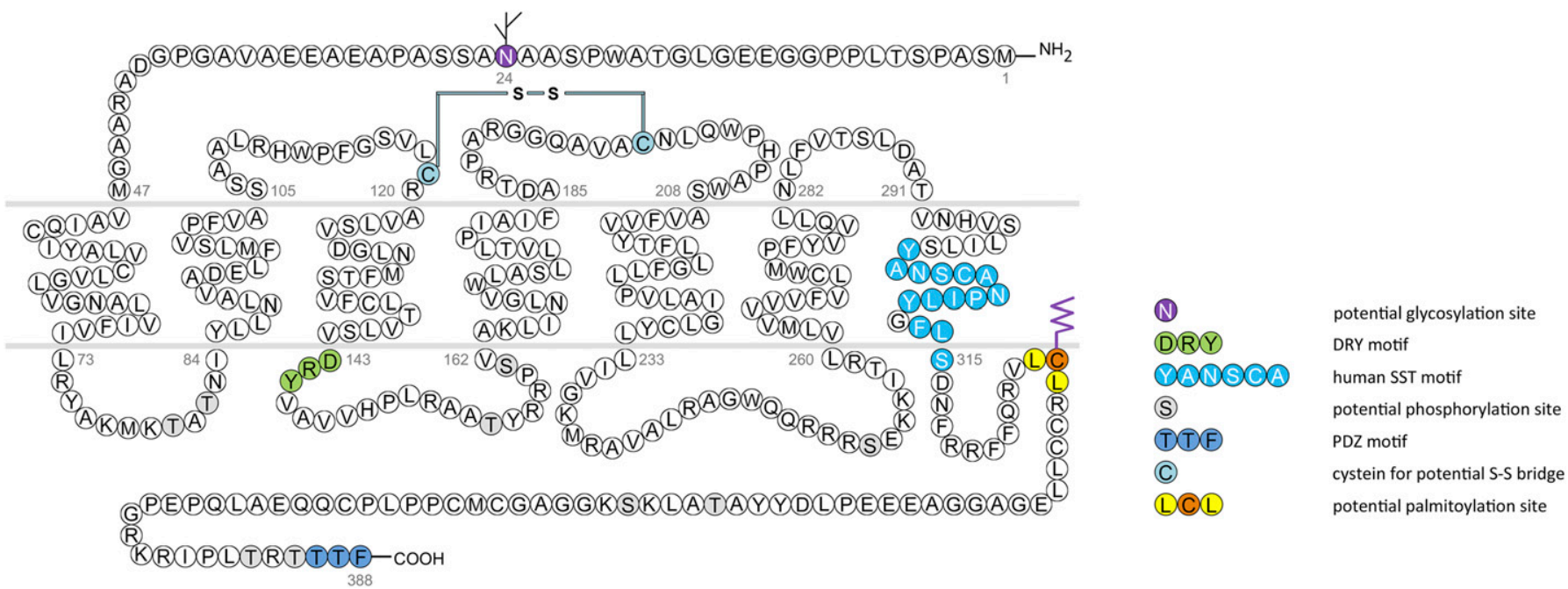

Fig. 15. Structure of human $\mathrm{SST}_{4}$. The primary and secondary amino acid structure of the human $\mathrm{SST}_{4}$ (UniProtKB - $\mathrm{P} 31391$ ) is shown in a schematic serpentine format. The glycosylation site is colored in purple; the DRY motif is highlighted in green; the human SST motif is in light blue; potential phosphorylation sites are in gray; the PDZ ligand motif is in dark blue; the disulfide-forming cysteines are in pale blue; and the potential palmitoylation site is in orange.

types in vitro, including rat pituitary cells. Elements located between -459 and -984 bp enhance promoter activity, although putative binding sites for tissue-specific transcription factors were not identified in these regions. Pathophysiological factors affecting SRIF and $\mathrm{SST}_{1-5}$ expression in endothelial cells include hypoxia, which

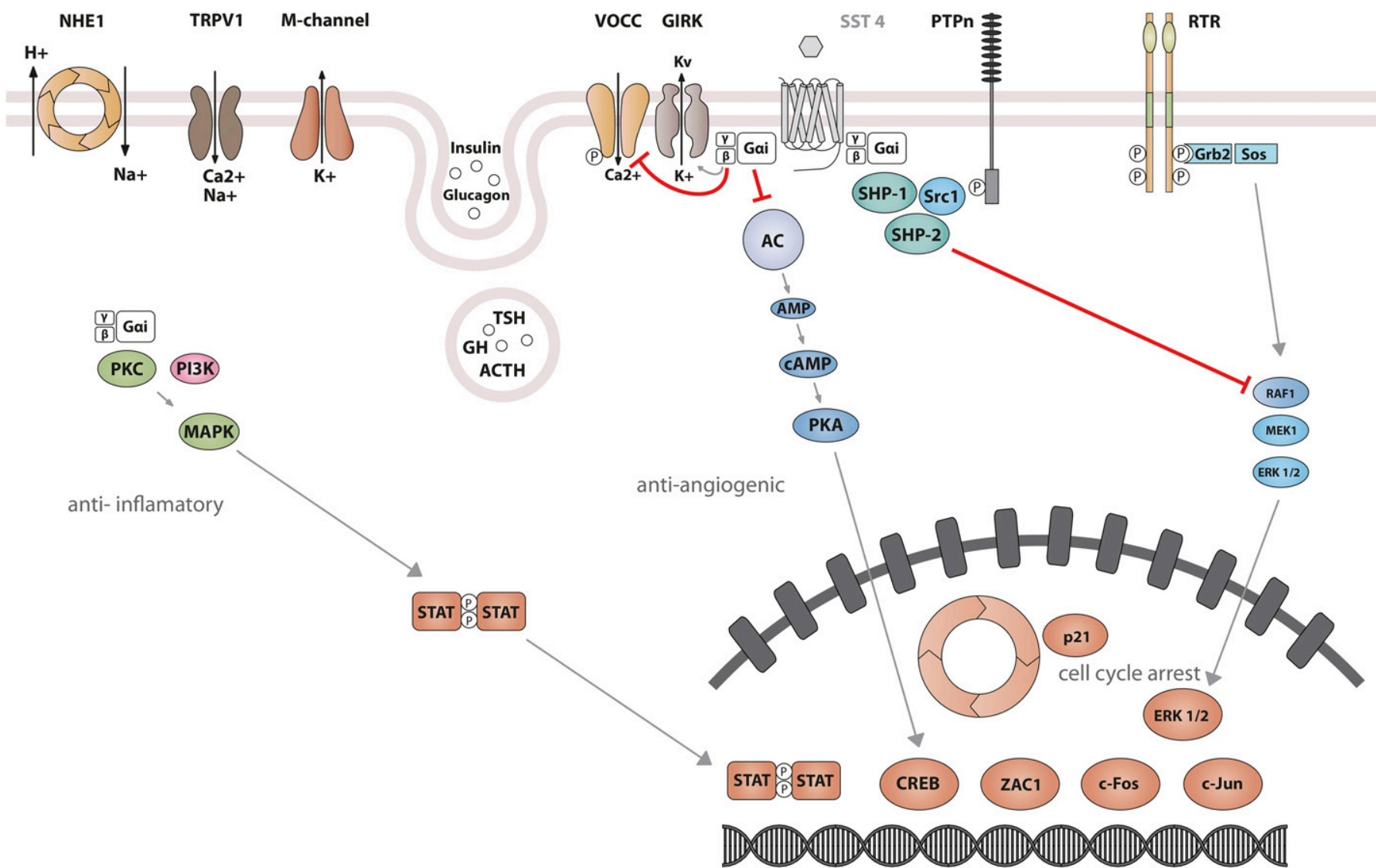

Fig. 16. $\mathrm{SST}_{4}$ signaling leading to inhibition of hormone secretion, proliferation, and migration. By coupling to Gi proteins, $\mathrm{SST}_{4}$ inhibits adenylate cyclase and reduces cAMP accumulation, and reduces intracellular $\mathrm{Ca}^{2+}$ concentrations by activating GIRK and M channels, which results in membrane hyperpolarization and subsequent reduction of $\mathrm{Ca}^{2+}$ and $\mathrm{Na}^{+}$influx through VOCC and TRPV1. In addition, $\mathrm{SST}_{4}$ inhibits the NHE1 activity, resulting in a decrease of extracellular acidification rate. Another major effector of $\mathrm{SST}_{4}$ is the tyrosine phosphatase SHP-2, which mediates antiproliferative effects. $\mathrm{SST}_{4}$ also mediates a prolonged ERK activation and subsequent signal transducer and activator of transcription 3 phosphorylation, which is Gi/Go and $\mathrm{PI} 3 \mathrm{~K}$ dependent. Activation of $\mathrm{SST}_{4}$ can induce cell cycle arrest by upregulation of the cyclin-dependent kinase inhibitor p21 (cip1/WAF1). 
induces $\mathrm{SST}_{4}$ mRNA expression in human umbilical vein endothelial cells (Dal Monte et al., 2011). In brain endothelial cells, proinflammatory cytokines and lipopolysaccharide upregulate expression of $\mathrm{SST}_{4}$ as well as $\mathrm{SST}_{2}$ (Basivireddy et al., 2013). Substantial differences between rat and the human $\mathrm{SST}_{4}$ have been reported in desensitization after prolonged SRIF treatment. In transfected CHO cells expressing human $\mathrm{SST}_{4}$, both activation of NHE1 and stimulation of ERK phosphorylation were susceptible to a marked desensitization in response to SRIF (Smalley et al., 1998, 1999; Engström et al., 2006).

\section{Somatostatin Receptor 4 Trafficking}

As to the fate of the receptor upon SRIF-14 binding, low levels of internalized human $\mathrm{SST}_{4}$ were detected in transfected cells. The use of radiolabeled ligand suggested rapid dissociation of the complex and rapid recycling of the ligand to the extracellular medium and of the receptor to the plasma membrane, respectively. These observations may suggest that sustained desensitization of human $\mathrm{SST}_{4}$ is not entirely dependent on receptor sequestration (Smalley et al., 2001). In contrast, rat $\mathrm{SST}_{4}$ is not susceptible to rapid desensitization and does not undergo internalization at all, as shown in transfected cells and in brain tissue after in vivo treatment of rats with SRIF (Kreienkamp et al., 1998; Schreff et al., 2000). Although a number of potential phosphate acceptor sites are present, rat $\mathrm{SST}_{4}$ is also not subject to agonist-induced phosphorylation and does not recruit $\beta$-arrestins to the plasma membrane when activated (Tulipano et al., 2004). Sitedirected mutagenesis allowed for identification of a single amino acid residue $\left(\mathrm{Thr}^{331}\right)$ in rat $\mathrm{SST}_{4}$, which confers resistance to agonist-induced internalization (Kreienkamp et al., 1998).

\section{E. Somatostatin Receptor 4 Interacting Proteins}

Direct interaction between $\mathrm{SST}_{4}$ and the scaffolding PSD-95 has been shown in transfected HEK293 cells and in hippocampal neurons (Christenn et al., 2007). This interaction is mediated by binding of a PDZ domain of PSD-95 to a PDZ-domain ligand motif in the C-terminal tail of $\mathrm{SST}_{4}$. PSD-95 is not involved in regulating receptor signaling in transfected cells. Moreover, as PSD-95 and $\mathrm{SST}_{4}$ partially colocalize in hippocampal neurons in the dendritic domain, the scaffold protein may be involved in targeting $\mathrm{SST}_{4}$, mainly localized in the somatodendritic postsynaptic domain in brain. In addition, an interaction between $\mathrm{SST}_{4}$ and the membrane glycoprotein dipeptidyl peptidase-4/cluster of differentiation 26 (CD26) occurs in malignant pleural mesothelioma cells. CD26 is a $110-\mathrm{kDa}$ type II membrane glycoprotein with known dipeptidyl peptidase IV activity in its extracellular domain. $\mathrm{SST}_{4}$ and CD26 are highly coexpressed and interact through their cytoplasmic domains in malignant pleural mesothelioma cells. The $\mathrm{SST}_{4}-\mathrm{CD} 26$ interaction reduces cytostatic effects of $\mathrm{SST}_{4}$ agonists (Yamamoto et al., 2014). Their efficacy was enhanced by suppression of CD26 as well as by treatment of cells with anti-CD26 mAbs. Upon treatment with anti-CD26 mAbs, $\mathrm{SST}_{4}$ aggregated preferentially in lipid rafts. Interestingly, SHP-2 also clustered in lipid rafts along with $\mathrm{SST}_{4}$, which in turn facilitated $\mathrm{SST}_{4}$-mediated cytostatic and antitumor effects. Moreover, in transfected HEK293 cells, human $\mathrm{SST}_{4}$ exists as constitutive homodimers and as constitutive heterodimers when coexpressed with human $\mathrm{SST}_{5}$ (Somvanshi et al., 2009). However, in a different cellular background (CHO-K1 cells), cotransfection of these two receptors did not result in heterodimerization under otherwise identical conditions (Rocheville et al., 2000b). Nevertheless, to what extent the $\mathrm{SST}_{4}-\mathrm{SST}_{5}$ interaction may be physiologically relevant is not known.

\section{F. Somatostatin Receptor 4 Anatomic Framework}

The $\mathrm{SST}_{4}$ is localized in diverse rat brain areas, and there is substantial consistency between mRNA and $\mathrm{SST}_{4}$-like immunoreactivity distribution in the rat (Fehlmann et al., 2000; Schreff et al., 2000; Schulz et al., 2000a; Stumm et al., 2004). High levels of $\mathrm{SST}_{4}$ are present throughout the rat forebrain, whereas the signal progressively decreased toward caudal brain regions. $\mathrm{SST}_{4}$ is abundantly expressed in the olfactory bulb and in other olfactory structures. $\mathrm{SST}_{4}$ is found throughout layers I-VI of the neocortex, in the hippocampus formation, the hilar region of the dentate gyrus, the amygdala, and the hypothalamus. $\mathrm{SST}_{4}$ immunoreactivity is distributed along neuronal processes in the striatum, nucleus accumbens, and globus pallidus. In addition, approximately $50 \%$ of DRG showed $\mathrm{SST}_{4}$-like immunoreactivity (Bär et al., 2004). $\mathrm{SST}_{4}$ is also abundantly present in retinal ganglion cells (Farrell et al., 2010). At the cellular and subcellular level of adult rat CNS, $\mathrm{SST}_{4}$ is preferentially distributed to somatodendritic neuronal domains. In the neocortex, hippocampus, and striatum, $\mathrm{SST}_{4}$ is almost exclusively confined to dendrites and symmetric synapses. In the hippocampus, targeting to asymmetric, excitatory synapses was observed. Colocalization studies of SRIF and $\mathrm{SST}_{4}$ provided evidence of close apposition of SRIFcontaining axons and their terminals with dendrites containing $\mathrm{SST}_{4}$, suggesting that $\mathrm{SST}_{4}$ mainly functions postsynaptically (Schreff et al., 2000). In the periphery, $\mathrm{SST}_{4}$ is expressed in the lung, heart, and placenta and is undetectable in pancreatic islets (Fehlmann et al., 2000; Ludvigsen et al., 2015). Given that highly specific rabbit mAbs are not yet available, the cellular expression of human $\mathrm{SST}_{4}$ is less well characterized. Using polyclonal antibodies, $\mathrm{SST}_{4}$ receptors have been found in cells of the bronchial glands, the exocrine pancreas, in the GIT (stomach and duodenum), in kidney tubules, and in the parathyroid gland (Taniyama et al., 2005). Lack of $\mathrm{SST}_{4}$ binding sites and immunoreactivity in hypophyseal 
tissue suggests that $\mathrm{SST}_{4}$ does not play a major role in SRIF-mediated neuroendocrine control of the human anterior pituitary (Panetta and Patel, 1995; Reubi et al., 2001). $\mathrm{SST}_{4}$ immunoreactivity in human brain was restricted to the gray matter in cerebral cortex areas. In the sensory and motor cortex, staining of the large motor neurons was not detected. Immunopositive pyramidal cells were found in cortical layers III-VI, in agreement with results of $\mathrm{SST}_{4}$-like immunoreactivity in rat neocortex layers III-V, most likely representing targets of local SRIFergic neurons in the cerebral cortex. $\mathrm{SST}_{4}$ protein was found in the hippocampal formation, with immunostaining of cell bodies and processes in the polymorphic layer of the dentate gyrus and in the thalamus, where it localized particularly in fibers. $\mathrm{SST}_{4}$ immunoreactivity was also observed in the cerebellar cortex and the medulla (Selmer et al., 2000). In summary, data on $\mathrm{SST}_{4}$ distribution in selected human brain areas correlate well with the distribution in rat brain. Comparison between $\mathrm{SST}_{4}$ immunoreactivity and $\mathrm{SST}_{4}$ mRNA distribution by in situ hybridization (Schindler et al., 1995; Thoss et al., 1996b; Piwko et al., 1997) in human brain showed some discrepancies in thalamus, cerebral cortex, and cerebellum expression patterns (Selmer et al., 2000). According to receptor autoradiography using selective ligands, $\mathrm{SST}_{4}$ cannot be frequently found in human tumors (Reubi et al., 2001). Finally, two studies reported divergent results on the expression of $\mathrm{SST}_{4}$ in human insulinomas. In the first study, immunoreactivity analysis suggested that $\mathrm{SST}_{4}$ was the most frequent receptor expressed in both benign and malignant insulinomas (Portela-Gomes et al., 2007). In the second study, the mRNA expression analysis and binding assays suggested that $\mathrm{SST}_{4}$ expression was limited to approximately $20 \%$ of tumors (Bertherat et al., 2003).

\section{G. Somatostatin Receptor 4 Function}

$\mathrm{SST}_{4}$ is expressed in areas involved in learning and memory processes. In mice, pharmacological studies suggest that activation of hippocampal $\mathrm{SST}_{4}$ leads to impaired spatial learning and enhanced cued memory. This effect suggests a switch from formation of hippocampus-based memory to striatum-based memory (Gastambide et al., 2010). In addition, behavioral studies using selective ligands showed that activation of $\mathrm{SST}_{4}$ in the striatum increases rat locomotor activity via glutamatergic systems (Raynor et al., 1993a; Santis et al., 2009). Finally, activation of $\mathrm{SST}_{4}$ in the CNS plays a role in modulation of behavioral responses to acute stress and of behavioral and neuroendocrine changes induced by mild chronic stress in mice, suggesting involvement of $\mathrm{SST}_{4}$ in anxiety and depression-like behavior (Scheich et al., 2016, 2017). Experimental data suggest $\mathrm{SST}_{4}$ as a therapeutic target in Alzheimer's disease. Administration of the $\mathrm{SST}_{4}$ agonist NNC269100 was found to reduce soluble amyloid- $\beta$ peptide oligomers in the brain by enhancing metalloproteinasemediated amyloid degradation in two different mouse models. This effect correlated with improved learning (Sandoval et al., 2012, 2013). Interestingly, $\mathrm{SST}_{4}$ expression levels are drastically reduced in the temporal cortex of female Alzheimer's disease patients (Gahete et al., 2010b). SRIF is also highly expressed in brain areas associated with seizures. Activation of $\mathrm{SST}_{4}$ suppressed epileptiform activity in mouse hippocampal slices and exerts anticonvulsant effects in vivo. Moreover, $\mathrm{SST}_{4} \mathrm{KO}$ mice showed increased susceptibility to limbic seizures (Qiu et al., 2008) but other studies suggested excitatory and proconvulsant effects of $\mathrm{SST}_{4}$ activation (Moneta et al., 2002). In a rat model for limbic seizures, intrahippocampal administration of a $\mathrm{SST}_{4}$-selective agonist has marked anticonvulsant effects, similar to administration of $\mathrm{SST}_{2}$ and $\mathrm{SST}_{3}$ agonists (Aourz et al., 2011). $\mathrm{SST}_{4}$ is currently being evaluated as a therapeutic target for development of anti-inflammatory and/or analgesic drugs without endocrine side effects (Sándor et al., 2006; Elekes et al., 2008; Helyes et al., 2009; Schuelert et al., 2015). Mice lacking $\mathrm{SST}_{4}$ exhibit increased inflammatory and nociceptive responses, suggesting impaired defense mechanisms (Helyes et al., 2009; Van Op den Bosch et al., 2009). Peripheral administration of selective $\mathrm{SST}_{4}$ agonists reduced formalin-induced acute nociception and mechanical allodynia in arthritic and neuropathic pain models and exhibited multiple antiinflammatory effects in rodents (Sándor et al., 2006; Schuelert et al., 2015). DRG neurons are most likely a primary target of $\mathrm{SST}_{4}$ agonists. $\mathrm{SST}_{4}$ activation reduces membrane excitability in DRG neurons by activating $\mathrm{K}_{\mathrm{ir}} 3 . \mathrm{x}$ and inhibiting VOCC channels, and both mechanisms are presumed to contribute to analgesic effects (Gorham et al., 2014a). $\mathrm{SST}_{4}$-selective agonists reduced acute and chronic airway inflammation as well as bronchial hyper-reactivity in the mouse, and inhibited carbachol-induced bronchoconstriction (Elekes et al., 2008).

\section{H. Somatostatin Receptor 4 Ligands}

Compared with other SST subtypes, $\mathrm{SST}_{4}$ displays somewhat lower affinity for the common endogenous ligand SRIF-14. Among multi-SST ligands, the cyclic heptapeptide veldoreotide (COR005) (previously called somatoprim, DG3173) is unique in that it binds to $\mathrm{SST}_{4}$ in addition to $\mathrm{SST}_{2}$ and $\mathrm{SST}_{5}$ (Shimon et al., 2004; Plöckinger et al., 2012). Veldoreotide is a potent suppressor of GH secretion from human pituitary adenomas, which is attributed to its affinity for $\mathrm{SST}_{2}$ and $\mathrm{SST}_{5}$. Interestingly, despite nanomolar affinity for $\mathrm{SST}_{5}$, veldoreotide has minimal effects on insulin secretion from endocrine pancreas in vivo. In phase II clinical trials for the treatment of acromegaly, veldoreotide proved to be effective when administered by s.c. bolus or s.c. infusion (ClinicalTrials.gov NCT02235987 and NCT02217800). TT-232 is a stable cyclic heptapeptide 
with partial activity at $\mathrm{SST}_{4}$ that also binds to $\mathrm{SST}_{1}$ (Crider and Witt, 2007). Compound J-2156, a nonpeptide agonist displaying high selectivity and high affinity for $\mathrm{SST}_{4}$ (Fig. 17; Table 7), was derived by solid-phase synthesis of a series of 1-naphthalenesulfonylaminopeptidomimetics (Engström et al., 2005). Both TT-232 and J-2156 exhibited anti-inflammatory and antinociceptive effects after i.p. administration in rodents (Crider and Witt, 2007). NNC26-9100 is the lead compound of another structurally distinct class of highly selective $\mathrm{SST}_{4}$ agonists (Liu et al., 1998). L-803,087 is a nonpeptidic agonist with high affinity and selectivity for $\mathrm{SST}_{4}$ (Fig. 17; Table 7) (Rohrer et al., 1998). Unexpectedly for peptide GPCRs, only $\mathrm{SST}_{4}$ agonists have been identified, and antagonists are not yet available. $\mathrm{SST}_{4}$ agonists able to penetrate the blood brain barrier would be of great interest.

\section{Somatostatin Receptor 5}

\section{A. Somatostatin Receptor 5 Structure}

Cloning of human $\mathrm{SST}_{5}$, the last subtype of the SST family to be cloned (Yamada et al., 1993; Panetta et al., 1994), was preceded by that of rat $\mathrm{SST}_{5}$ (O'Carroll et al., 1992), which was initially termed rat SSTR4, due to the temporal proximity with the cloning of the receptor currently known as rat $\mathrm{SST}_{4}$ (Xu et al., 1993). After an initial period of confusion, the current nomenclature was agreed upon (Hoyer et al., 1995a), and subsequent studies led to detailed characterization of $\mathrm{SST}_{5}$. The human SSTR5 gene is localized on chromosome $16 \mathrm{p} 13.3$, and its coding sequence spans a single exon, encoding a protein of 364 amino acids (Panetta et al., 1994; Takeda et al., 1995), whereas the rat Sstr5 receptor gene encodes a protein of 363 amino acids (O'Carroll et al., 1992). Genes encoding mouse and rat $\mathrm{SST}_{5}$ are localized on chromosomes $17 \mathrm{~A} 3.3$ and 10q12, respectively. Mouse $\mathrm{SST}_{5}$ was also shown to encode a protein whose length appears to vary from 362 amino acids (Moldovan et al., 1998; Gordon et al., 1999) to 363 residues (Lublin et al., 1997; Feuerbach et al., 2000), and up to 385 amino acids (Baumeister et al., 1998), differences attributed to cloning procedures or mouse strain. Initial comparative analysis had revealed that the sequence of $\mathrm{SST}_{5}$ is evolutionarily well conserved, with human $\mathrm{SST}_{5}$ showing $80 \%$ homology with the amino acid sequence of rat and mouse $\mathrm{SST}_{5}$ (O'Carroll et al., 1992; Lublin et al., 1997). Nevertheless, compared with other SSTs, human $\mathrm{SST}_{5}$ seems to display lower levels of identity and similarity with $\mathrm{SST}_{5}$ from other species, particularly at the carboxylterminal tail, as well as with other human SSTs [ranging from $42 \%$ to $52 \%$ compared with $\mathrm{SST}_{1}, \mathrm{SST}_{2}$, $\mathrm{SST}_{3}$, and $\mathrm{SST}_{4}$ (O'Carroll et al., 1992; Panetta et al., 1994; Møller et al., 2003)], which portrays $\mathrm{SST}_{5}$ as the least conserved subtype among SSTs. Original cloning revealed two potential $N$-glycosylation sites in human $\mathrm{SST}_{5}$, located at Asn-13 and Asn-26 in the aminoterminal segment, and a third Asn-187 in the second ICL (Fig. 18) (Yamada et al., 1993; Panetta et al., 1994). Likewise, the 385 -amino-acid mouse $\mathrm{SST}_{5}$ described by Baumeister et al. (1998) contains three equivalent putative $N$-glycosylation sites at residues 36,46 , and 208. The estimated molecular mass of the 364-residue human $\mathrm{SST}_{5}$ is $39 \mathrm{kDa}$, whereas immunodetection of $\mathrm{SST}_{5}$ from transfected baby hamster kidney cells in Western blot showed several bands ranging from 52 to $66 \mathrm{kDa}$. Deglycosylation with PNGase F rendered a single band with an estimated $40-\mathrm{kDa}$ mass, thus confirming the glycoprotein nature of the receptor (Helboe et al., 1997). Human $\mathrm{SST}_{5}$ contains a cysteine residue at position 320 as a presumed palmitoylation anchor, the mammalian SST signature motif in transmembrane region $7\left({ }^{294}\right.$ YANSCANPVLY $\left.{ }^{304}\right)$, and two cysteine residues at residues 112 in the first extracellular loop (ECL) and 186 in the second ECL, predicted to enable a disulfide bridge (Reisine and Bell, 1995). Studies on structural determinants of human $\mathrm{SST}_{5}$ function using mutational analysis suggested that ECL 2 is key to form the receptor ligand-binding pocket (Greenwood et al., 1997).

In contrast to other SSTs, a number of studies have identified single-nucleotide polymorphisms in the human $\mathrm{SST}_{5}$ gene that may imply potential pathophysiological functions in pancreatic NETs and other cancers (Li et al., 2011; Zhou et al., 2011, 2012), bipolar affective disorder (Nyegaard et al., 2002), acromegaly (Lania et al., 2008; Ciganoka et al., 2011), prostate cancer (Hormaechea-Agulla et al., 2017), and in the regulation of circulating IGF-1 and IGFBP3 in prostate and breast cancer ( $\mathrm{Gu}$ et al., 2010). However, only a single $\mathrm{SST}_{5}$

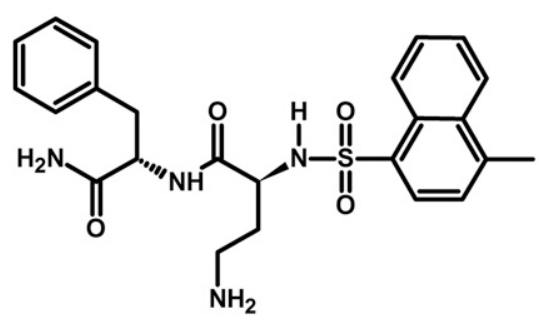

$\mathrm{J}-2156$

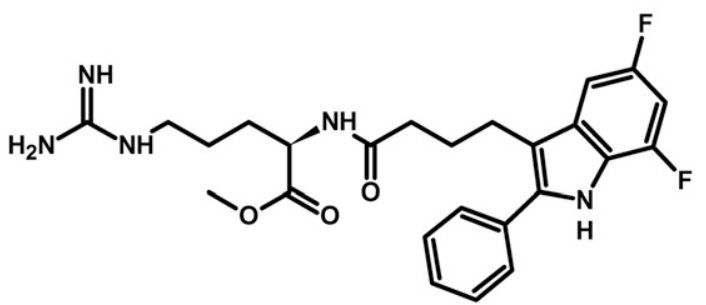

L-803,087

Fig. 17. Structures of synthetic $\mathrm{SST}_{4}$ ligands. J-2156 and L-803,087, $\mathrm{SST}_{4}$ agonists. 
TABLE 7

Ligand-binding affinities of $\mathrm{SST}_{4}$-selective ligands

\begin{tabular}{lccccr}
\hline & SST $_{1}$ & SST $_{2}$ & SST $_{3}$ & SST $_{4}$ & SST $_{5}$ \\
\hline${\mathrm{L}-803,087^{a}}$ & 199 & 4720 & 1280 & 0.7 & 3880 \\
$\mathrm{~J}^{2} 156^{b}$ & 350 & $>5000$ & 1300 & 0.8 & 460
\end{tabular}

${ }^{a}$ Data from Rohrer et al. (1998).

${ }^{b}$ Data from Engström et al. (2005).

mutation associated with acromegaly has been described in a single patient (Ballare et al., 2001).

The human SSTR5 gene as well as rodent orthologs uniquely undergoes noncanonical splicing to truncated variants that possess less than the typical seven TMDs that characterize all GPCRs (Cordoba-Chacon et al., 2011). In particular, two truncated human $\mathrm{SST}_{5}$ receptors exist, termed $\mathrm{SST}_{5} \mathrm{TMD}_{4}$ and $\mathrm{SST}_{5} \mathrm{TMD} 5$, which display distinct tissue distribution, subcellular localization, response to ligands, and signaling capacities as compared with canonical full-length $\mathrm{SST}_{5}$ (CordobaChacon et al., 2011). The most studied variant, $\mathrm{SST}_{5} \mathrm{TMD} 4$, is scarcely present in normal tissues but abundantly expressed in a number of tumors, including pituitary adenomas (Durán-Prado et al., 2009, 2010; Gatto et al., 2013a; Luque et al., 2015), breast cancer
(Durán-Prado et al., 2012b; Gahete et al., 2016), thyroid cancer (Puig-Domingo et al., 2014), medullary thyroid carcinoma (Mole et al., 2015), NETs (Sampedro-Nunez et al., 2016), and prostate cancer (Hormaechea-Agulla et al., 2017). In all those tumor types, $\mathrm{SST}_{5} \mathrm{TMD} 4$ expression is associated with features of enhanced tumor aggressiveness that vary depending on the type of tumor: increased cell survival/proliferation, migration, invasion, angiogenesis, decreased apoptosis, poor response to octreotide/lanreotide, etc. Similar, albeit not identical, truncated $\mathrm{SST}_{5}$ receptor variants have also been cloned and characterized in pig (Durán-Prado et al., 2012a) and rodents (Cordoba-Chacon et al., 2010). Truncated $\mathrm{SST}_{5}$ receptor variants share a number of features, as follows: 1) preferential intracellular distribution (rather than the predominant plasma membrane localization of full-length $\mathrm{SST}_{5}$ ); 2) functional capacity to selectively respond to ligands (e.g., SRIF for $\mathrm{SST}_{5} \mathrm{TMD} 5$; CST for $\left.\mathrm{SST}_{5} \mathrm{TMD} 4\right)$, by modulating distinct signaling pathways (cAMP, $\mathrm{Ca}^{2+}$, etc.); and 3) ability to physically interact with full-length $\mathrm{SST}_{2}$ and/or $\mathrm{SST}_{5}$, to retain them in intracellular compartments, and, eventually, disrupt their normal function. As a result, it has been proposed that $\mathrm{SST}_{5} \mathrm{TMD} 4$ and
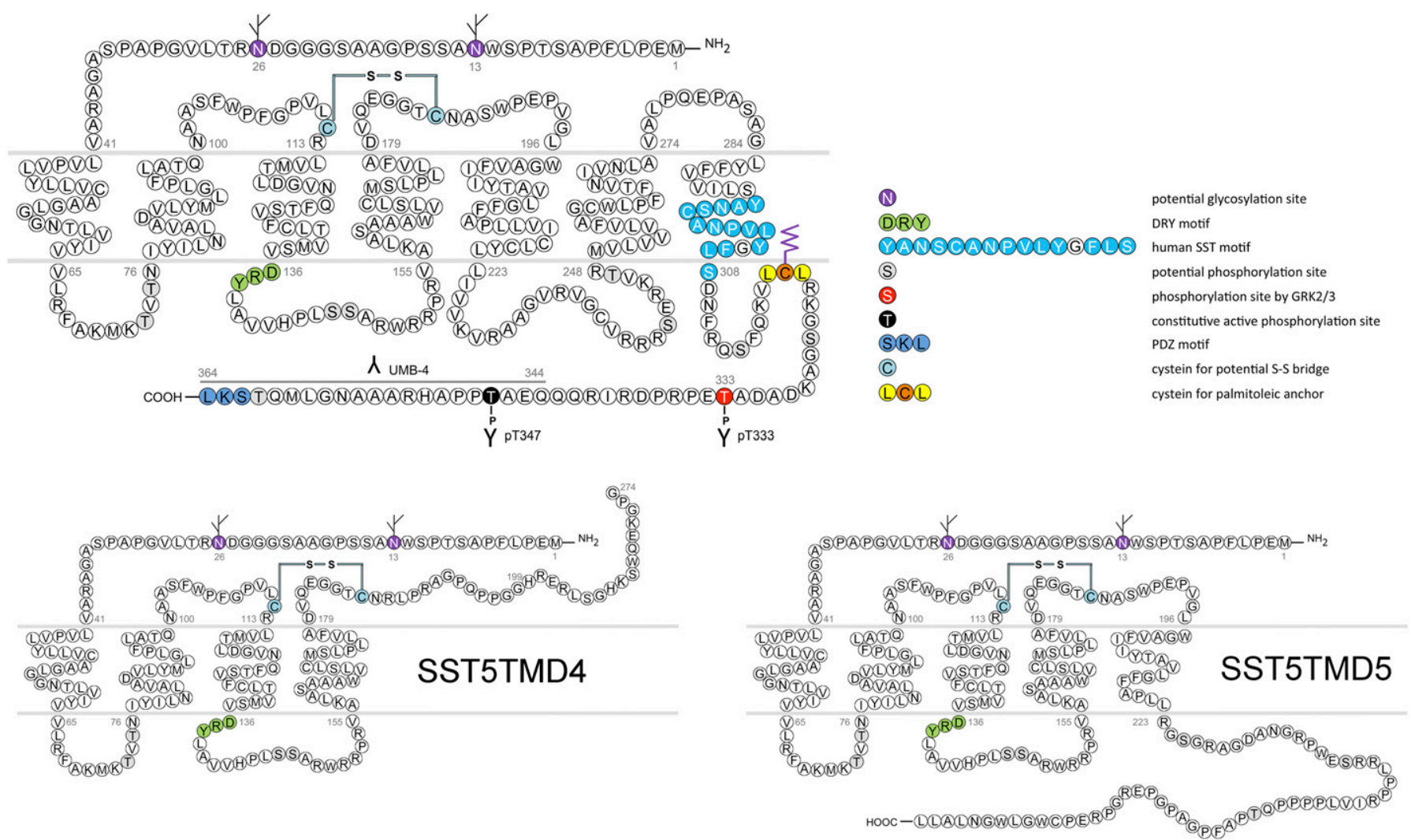

Fig. 18. Structure of human $\mathrm{SST}_{5}$. The primary and secondary amino acid structure of the human $\mathrm{SST}_{5}$ (UniProtKB - P35346) as well as its truncated variants $\mathrm{SST}_{5}$ TMD4 and $\mathrm{SST}_{5}$ TMD5 are shown in a schematic serpentine format. Glycosylation sites are colored in purple; the DRY motif is highlighted in green; the human SST motif is in light blue; potential phosphorylation sites are in gray; identified GRK2/3 phosphorylation site is in red; constitutive phosphorylation site is in black; the PDZ ligand motif is in dark blue; the disulfide-forming cysteines are in pale blue; and the potential

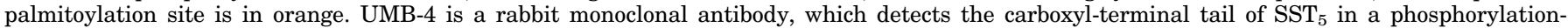
independent manner. 
other truncated variants may act as functional dominant-negative partners for their respective fulllength $\mathrm{SST}_{2}$ and $\mathrm{SST}_{5}$ receptor counterparts (CordobaChacon et al., 2011).

\section{B. Somatostatin Receptor 5 Signaling Mechanisms}

$\mathrm{SST}_{5}$ signals through a wide array of mechanisms, which include prototypical $\mathrm{G}_{\alpha \mathrm{i}}$-dependent inhibition of adenylyl cyclase common to all SSTs, regulation of other crucial enzymes like PTPs and MAPK, as well as modulation of free cytosolic calcium and potassium concentrations (Fig. 19) (Møller et al., 2003; Peverelli et al., 2009, 2013; van der Hoek et al., 2010; Theodoropoulou and Stalla, 2013). Original cloning studies showed that both human and rat $\mathrm{SST}_{5}$ inhibited forskolin-stimulated cAMP accumulation through a $\mathrm{G}_{\alpha \mathrm{i}}$ protein-dependent mechanism (Yamada et al., 1993; Panetta et al., 1994). These functions were confirmed in subsequent studies, mainly using $\mathrm{SST}_{5^{-}}$ transfected cells and/or selective ligands, which revealed that $\mathrm{SST}_{5}$ activates additional pathways in a context (i.e., ligand, cell environment)- and species-dependent manner (Siehler and Hoyer, 1999b,c; Møller et al., 2003; van der Hoek et al., 2010; Theodoropoulou and Stalla, 2013). Thus, $\mathrm{SST}_{5}$ activates PLC activity, thereby increasing cytosolic $\mathrm{Ca}^{2+}$ levels by release from intracellular stores (Wilkinson et al., 1997a,b; Siehler and Hoyer, 1999c). However, $\mathrm{SST}_{5}$ also blocks VOCCs, thereby decreasing $\mathrm{Ca}^{2+}$ cell entry and cytosolic levels (Tallent et al., 1996), likely by hyperpolarizing the cell through $\mathrm{K}^{+}$influx via activation of $\mathrm{K}_{\mathrm{ir}} 3 . \mathrm{x}$ (Kreienkamp et al., 1997; Smith et al., 2001). Using wild-type and mutant $\mathrm{SST}_{5}$, the main signaling pathways activated by this receptor have been delineated, as follows: 1) $\mathrm{SST}_{5}$ couples to individual $\mathrm{G}_{\alpha \mathrm{i} 1-3}$ and $\mathrm{G}_{\alpha \mathrm{oA}, \mathrm{B}}$; 2) $G_{\alpha o}$ mediates antisecretory and antimitogenic effects of $\mathrm{SST}_{5}$ in human pituitary somatotrophs; 3) the DRY motif is crucial for $\mathrm{SST}_{5}$ coupling with downstream effectors, whereas the BBXXB motif within the third ICL is dispensable for cAMP inhibition but essential for $\mathrm{SST}_{5}$ actions to reduce intracellular calcium levels and inhibit ERK1/2 activation, as well as for $\beta$-arrestin/receptor interaction and receptor internalization; and 4) residues 328-347 within the $\mathrm{C}$ terminus may play an inhibitory role in receptor internalization (Ballare et al., 2001; Peverelli et al., 2008, 2009, 2013). In fact, earlier mutational analysis indicated that Cys320 and, by and large, the C-tail of $\mathrm{SST}_{5}$ are essential for functional effector coupling (e.g., to adenylyl cyclase) and agonistinduced receptor desensitization and internalization (Hukovic et al., 1998).

$\mathrm{SST}_{5}$ inhibits ERK1/2 by a dephosphorylation cascade, including inhibition of guanylate cyclase and inhibition of cGMP-dependent protein kinase G (Cordelier et al., 1997). It also exerts antiproliferative effects by activating neuronal NOS via p60src kinase (Cordelier et al., 2006). $\mathrm{SST}_{5}$ activates stress-activated protein kinase or c-Jun $\mathrm{N}$-terminal kinase via $\mathrm{G} \alpha_{12}$ proteins (Komatsuzaki et al., 2001). In addition, it activates and upregulates $\mathrm{N}$-methyl-D-aspartate receptor function by a mechanism involving calmodulin-dependent kinase II, PLC, protein kinase $\mathrm{C}$, and tyrosine kinases in hippocampal

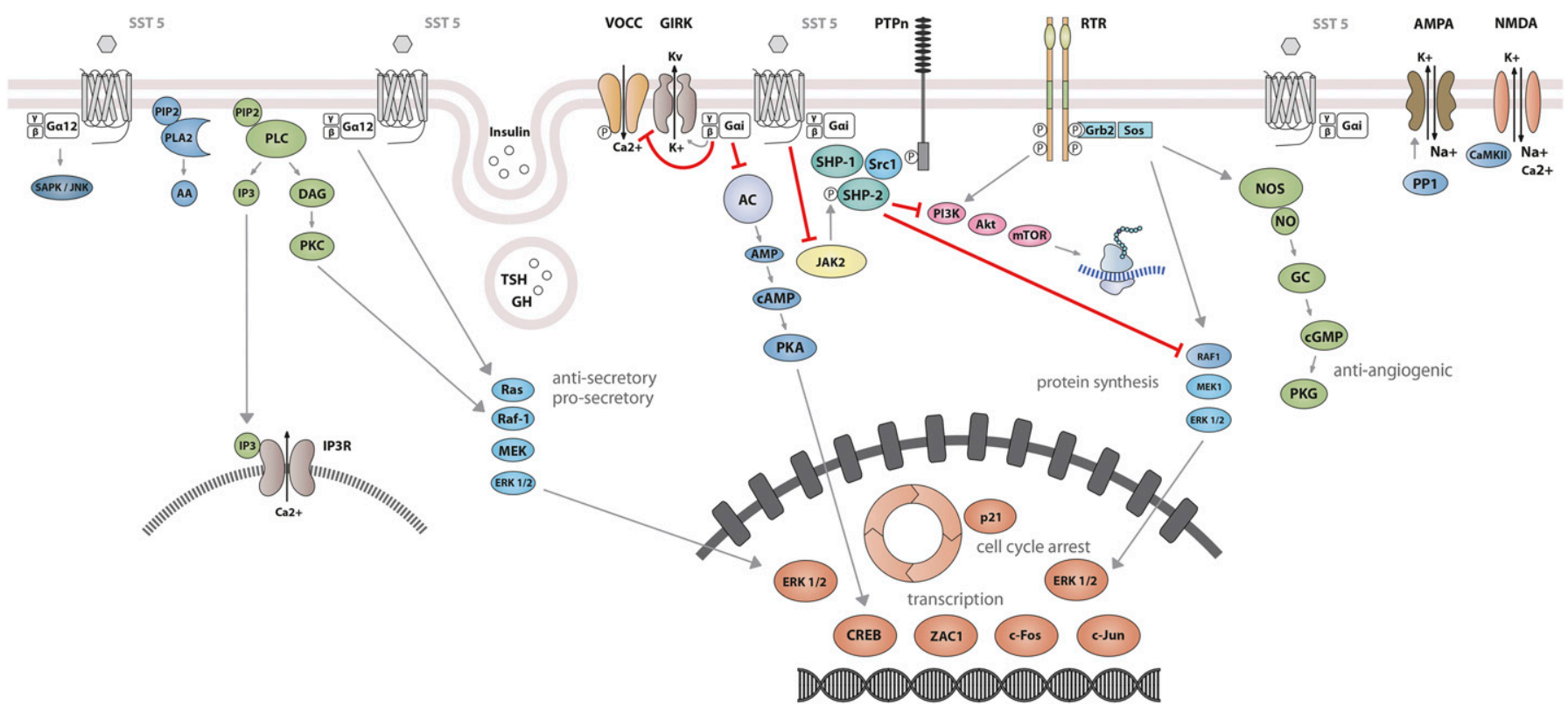

Fig. 19. $\mathrm{SST}_{5}$ signaling leading to inhibition of hormone secretion and proliferation. By coupling to Gi proteins, $\mathrm{SST}_{5}$ inhibits adenylate cyclase and reduces cAMP accumulation, and reduces intracellular $\mathrm{Ca}^{2+}$ concentrations by activating GIRK channels, which results in membrane hyperpolarization and subsequent reduction of $\mathrm{Ca}^{2+}$ influx through VOCC. This results in decreased hormone secretion. By coupling to a pertussis toxin-independent G protein, $\mathrm{SST}_{5}$ activates $\mathrm{PLC}$, triggering inositol-1,4,5-trisphosphate $\left(\mathrm{IP}_{3}\right)$ production and subsequent Ca ${ }^{2+}$ release into the cytoplasm from endoplasmic reticulum. Major downstream effectors of SST $_{5}$ are the tyrosine phosphatases SHP-1 and SHP-2, which subsequently inhibit mTOR pathway, thereby decreasing cell growth and proliferation. In addition, SST $_{5}$ inhibits NHE1 activity, resulting in a decrease of extracellular acidification rate. 
noradrenergic nerve endings (Pittaluga et al., 2005). In rat retinal ganglion cells, $\mathrm{SST}_{5}$ mediates suppression of 2-amino-3-(5-methyl-3-oxo-2,3-dihydro-1,2-oxazol4-yl)propanoic acid (AMPA) responses by acting through a signaling cascade involving $\mathrm{G}_{\mathrm{i} / 0} / \mathrm{cAMP}-\mathrm{PKA} /$ ryanodine/ $\mathrm{Ca}^{2+} / \mathrm{CAM} /$ calcineurin/PP1 (Deng et al., 2016).

$\mathrm{SST}_{5}$ displays constitutive activity, resulting in tonic inhibition of cAMP and ERK1/2 signaling and thus reducing hormone secretion (Ben-Shlomo et al., 2007, 2009b; Ben-Shlomo and Melmed, 2010). Finally, it has been shown that very low concentrations of SRIF actually stimulate $\mathrm{GH}$ release from pig and nonhuman primate somatotrophs mediated via $\mathrm{SST}_{5}$ and involving adenylyl cyclase, cAMP/PKA, and intracellular $\mathrm{Ca}^{2+}$ pathways (Luque et al., 2006; Cordoba-Chacon et al., $2012 \mathrm{~b}$ ). These actions may also be related to the presence of truncated $\mathrm{SST}_{5}$ receptor variants, which activate a wide array of signaling routes and molecular effectors, in a cell-, species-, and tumor-dependent manner, including cAMP, intracellular $\mathrm{Ca}^{2+}$, mitogen-activated protein kinases (ERK/c-Jun N-terminal kinase), AKT, cyclin D3, actin-related protein 2/3, MYC/myc-associated factor X, Wingless/int-1, and retinoblastoma protein signaling components (Durán-Prado et al., 2009, 2012a,b; Cordoba-Chacon et al., 2010; HormaecheaAgulla et al., 2017).

\section{Somatostatin Receptor 5 Regulation}

1. Regulation of Somatostatin Receptor 5 Gene Expression. The mouse Sstr5 gene contains two introns in the $5^{\prime}$-flanking region, which would enable the potential use of alternative gene promoters (Gordon et al., 1999; Baumeister and Meyerhof, 2000a; Olias et al., 2004). In humans, cell-specific expression was initially assigned to the first $900 \mathrm{bp}$ of the SSTR5 gene (Greenwood et al., 1994; Baumeister and Meyerhof, 2000a), but a subsequent study identified a $6.1-\mathrm{kb}$ intron in the $5^{\prime}$-UTR that unveiled a new upstream promoter, which can drive tissue-specific activation of the gene in pituitary in a Pit1-independent manner, but not in other tissues (e.g., small intestine, lung, or placenta) (Petersenn et al., 2002a). Similar to the mouse Sstr5 gene promoter, the human SSTR5 promoter lacks consensus sites for TATA or CAAT boxes, YY1, or a comparable initiator sequence, but contains relevant regulatory elements, including an essential GC-rich region containing SP1 binding sites, located proximal upstream of the transcription start site, two thyroid hormone response elements (between -1741 and -1269 and -317 and -101), and a cAMP-responsive element (between -101 and transcription start site). Indeed, reporter assays confirmed that forskolin and thyroid hormones enhance and SRIF inhibits promoter activity, which was not altered by other treatments, including IGF-1, estrogens, glucocorticoids, and phorbol 12-myristate 13-acetate (Greenwood et al., 1994; Petersenn et al., 2002a). Additional putative binding sites were identified for basic and tissue-specific transcription factors [e.g., nuclear factor 1, SP1, octamer-binding transcription factor 1, AP-1, AP-2, pituitary-specific positive transcription factor 1, Krox, pancreas-specific transcription factor 1 , $\mathrm{MyoD}$ ] and for hormone-dependent regulation [e.g., the cAMP-responsive element and tetracycline-responsive promoter element mentioned above, retinoic acid receptor, estrogen receptor, glucocorticoid receptor sites, etc.], as well as two CpG islands (Greenwood et al., 1994; Petersenn et al., 2002a).

Expression of the SSTR5 receptor gene is under multifactorial regulation that includes the following: 1) homologous control by SRIF and its analogs; 2) heterologous regulation by key stimulatory hypothalamic hormones, such as GHRH and ghrelin, which commonly inhibit SSTR5 receptor expression (Luque et al., 2004; Cordoba-Chacon et al., 2012a); and 3) endocrine-metabolic control by hormones from the major regulatory axes, such as sex steroids ( $17 \beta$-estradiol, testosterone), thyroid hormones, and glucocorticoids (reviewed in Baumeister and Meyerhof, 2000a; Olias et al., 2004; Ben-Shlomo and Melmed, 2010).

2. Ligand-Dependent Regulation of Somatostatin Receptor 5. The $\mathrm{SST}_{5}$ carboxyl-terminal tail contains only two potential phosphorylation sites at residues T333 and T347 (compared with the seven putative phosphate-acceptor sites in $\mathrm{SST}_{2}$ ), which seem to undergo markedly divergent dynamics: whereas T347 is constitutively phosphorylated even in the absence of ligand, T333 is phosphorylated by $\mathrm{GRK}_{2}$ immediately after agonist binding, as shown with phosphositespecific antibodies (Petrich et al., 2013; Schulz et al., 2014), and as supported by mutagenesis studies, which also point to T333 as an essential residue for $\mathrm{SST}_{5}$ receptor internalization (Peverelli et al., 2008). This latter study also suggested that the third ICL of $\mathrm{SST}_{5}$ is key for $\beta$-arrestin binding and receptor internalization upon ligand exposure, whereas the 36 terminal residues of the carboxyl-terminal tail may contribute to inhibit receptor internalization. Actually, regulation of $\mathrm{SST}_{5}$ by agonist-induced phosphorylation is tightly coupled to internalization and trafficking, for $\beta$-arrestin is recruited immediately after agonist-induced T333 phosphorylation and the receptor is subsequently internalized, in contrast to $\mathrm{SST}_{2}$, the $\mathrm{SST}_{5}-\beta$-arrestin complex is quickly disrupted, and $\mathrm{SST}_{5}$ traffics to early endosomes without $\beta$-arrestin (Petrich et al., 2013; Schulz et al., 2014). As a likely consequence of these distinct dynamics, the proportion of $\mathrm{SST}_{5}$ internalized after 30 minutes of SRIF exposure is considerably lower than that observed for the $\mathrm{SST}_{2}$ receptor $(30 \%-40 \%$ versus 80\%-90\%, respectively) (Petrich et al., 2013; Schulz et al., 2014). Nevertheless, it is important to emphasize that the dynamics of $\mathrm{SST}_{5}$ phosphorylation (and also its trafficking) are ligand- and context-dependent. Although SRIF-14 induces rapid dose-dependent $\mathrm{SST}_{5}$ phosphorylation, octreotide did not cause this effect. In addition, 
the $\mathrm{SST}_{5}$-selective agonist L-817,818 or the multireceptor ligand pasireotide induced $\mathrm{SST}_{5}$ phosphorylation to a lesser extent than the natural ligand SRIF-14, which was only paralleled by the $\mathrm{SST}_{5}$-selective agonist BIM-23268 (Shimon et al., 1997b; Petrich et al., 2013; Schulz et al., 2014). Additional mechanisms influencing agonist-dependent $\mathrm{SST}_{5}$ phosphorylation remain to be elucidated.

The reverse process of $\mathrm{SST}_{5}$ dephosphorylation at T333 is driven by $\mathrm{PP} 1 \gamma$ and depends on sequences in the carboxy-terminal tail, and is more rapid for $\mathrm{SST}_{5}$ than for $\mathrm{SST}_{2}$ (Petrich et al., 2013; Lehmann et al., 2014a; Schulz et al., 2014). In contrast, mechanisms that operate constitutive phosphorylation of T347 at $\mathrm{SST}_{5}$ and its physiologic relevance in vivo, if any, are still to be elucidated (Schulz et al., 2014). Likewise, possible ubiquitin-mediated degradation of $\mathrm{SST}_{5}$ remains to be elucidated, although recent results showed a relationship between the abundance and functionality of ubiquitin-specific peptidase 8 and the degree of $\mathrm{SST}_{5}$ expression at both mRNA and protein levels, which could bear clinical relevance for treating corticotroph adenomas (Hayashi et al., 2016).

\section{Somatostatin Receptor 5 Trafficking}

Trafficking of $\mathrm{SST}_{5}$ is intimately related to both ligand-dependent regulation of the receptor and interaction with specific proteins, which may include heterodimerization with other plasma membrane receptors. Thus, specific features of $\mathrm{SST}_{5}$ that reside within its sequence, phosphorylation/dephosphorylation kinetics, and interaction with $\beta$-arrestin and PDZ proteins are determinants for particular trafficking dynamics of this receptor. Little is known regarding the pathway of $\mathrm{SST}_{5}$ from its synthesis in the endoplasmic reticulum to the plasma membrane (i.e., export pathway), whereas trafficking involved in ligandinduced internalization and recycling has been studied in more detail (Fig. 20) (Jacobs and Schulz, 2008; Csaba et al., 2012; Schulz et al., 2014). Earlier studies explored the process of human and rat $\mathrm{SST}_{5}$ internalization and trafficking using different cell models (Hukovic et al., 1996, 1998; Roosterman et al., 1997; Roth et al., 1997b; Stroh et al., 2000b). In COS-7 cells, detailed microscopic and functional examination of SRIF-driven $\mathrm{SST}_{5}$ trafficking revealed that ligand exposure induced rapid (minutes) internalization of $\mathrm{SST}_{5}$, which involved trafficking to endosomal compartments, and was followed by a subsequent process of ligand-receptor dissociation and receptor recycling to the cell surface, accompanied by recruitment of receptors residing in an intracellular pool to the plasma membrane (Stroh et al., 2000b). Subsequent studies have dissected the contribution of specific protein regions and amino acid residues in $\mathrm{SST}_{5}$ trafficking, by demonstrating, for example, the importance of the third ICL (particularly phosphorylation of S242) and the carboxyl-terminal tail for agonist-induced internalization of $\mathrm{SST}_{5}$, which seems to be species and cell context dependent (Hukovic et al., 1998; Jacobs and Schulz, 2008; Peverelli et al., 2008; Csaba et al., 2012). In fact, $\mathrm{SST}_{5}$-trafficking dynamics are also heavily liganddependent, a feature of critical importance from a clinical standpoint. Trafficking does not solely depend on ligandbinding affinity for $\mathrm{SST}_{5}$, but on alternative properties that may entail, among others, distinct agonist-receptor binding sites, specific processes of phosphorylation, and subsequent activation of downstream interactions with $\beta$-arrestin, PDZ, and other interacting proteins (Cescato et al., 2006, 2012; Ginj et al., 2008; Jacobs and Schulz, 2008; Peverelli et al., 2008; Lesche et al., 2009; Petrich et al., 2013; Lehmann et al., 2014a; Schulz et al., 2014).

\section{E. Somatostatin Receptor 5 Interacting Proteins}

Like the other SSTs, $\mathrm{SST}_{5}$ contains a potential C-terminal class I PDZ ligand motif. Human and rodent $\mathrm{SST}_{5}$ interact with the PDZ domain protein interacting specifically with Tc10 (PIST, a Golgi-associated protein also known as Golgi-associated PDZ and coiled-coil motif-containing protein) and with sodium/hydrogen exchanger regulatory factor (NHERF)3/PDZ-K1 (PDZ protein expressed in kidney 1), a scaffold protein (Wente et al., 2005a,b; Csaba et al., 2012; Bauch et al., 2014). Analysis of $\mathrm{SST}_{5}-\mathrm{PIST}$ interaction in HEK293, AtT20, and MIN6 cells suggested that PIST may accompany $\mathrm{SST}_{5}$ to the Golgi/TGN, and also, that it may contribute to recycling to the plasma membrane (Wente et al., 2005a,b; Csaba et al., 2012). More recent work identified additional PDZ domain proteins interacting with mouse $\mathrm{SST}_{5}$, such as sorting nexin family member 27 and NHERF1, and further delineated the function of PIST, which seems to retain $\mathrm{SST}_{5}$ at the Golgi/TGN compartment. In contrast, NHERF1 could release the receptors from this area and thereby facilitate access to the cell surface (Bauch et al., 2014). PDZ-K1/NHERF3 appears to regulate specific interaction and functional activation of PLC $\beta 3$ by $\mathrm{SST}_{5}$ and other SSTs in response to SRIF by forming a ternary complex with PLC $\beta 3$ and SSTs (Kim et al., 2012). Thus, although the PDZ motif of $\mathrm{SST}_{5}$ does not seem to be indispensable for agonist-induced internalization of the receptor or for recycling to the plasma membrane, it may limit lysosomal degradation (and hence increase receptor stability) and enable additional signaling capabilities through selective PDZ domain-driven interactions (Wente et al., 2005a,b; Csaba et al., 2012; Kim et al., 2012; Bauch et al., 2014).

$\mathrm{SST}_{5}$ displays the ability to interact with other receptors from the SST family, forming homodimers/homomers or heterodimers/heteromers. Evidence in support of the existence and functional relevance of $\mathrm{SST}_{5}$ homodimers as well as heteromers with $\mathrm{SST}_{1}$ and $\mathrm{SST}_{2}$ has been derived in cell models, and these have delineated some molecular determinants and mechanisms involved in these interactions (Rocheville et al., 2000b; Durán-Prado et al., 2008; Grant et al., 2008; 


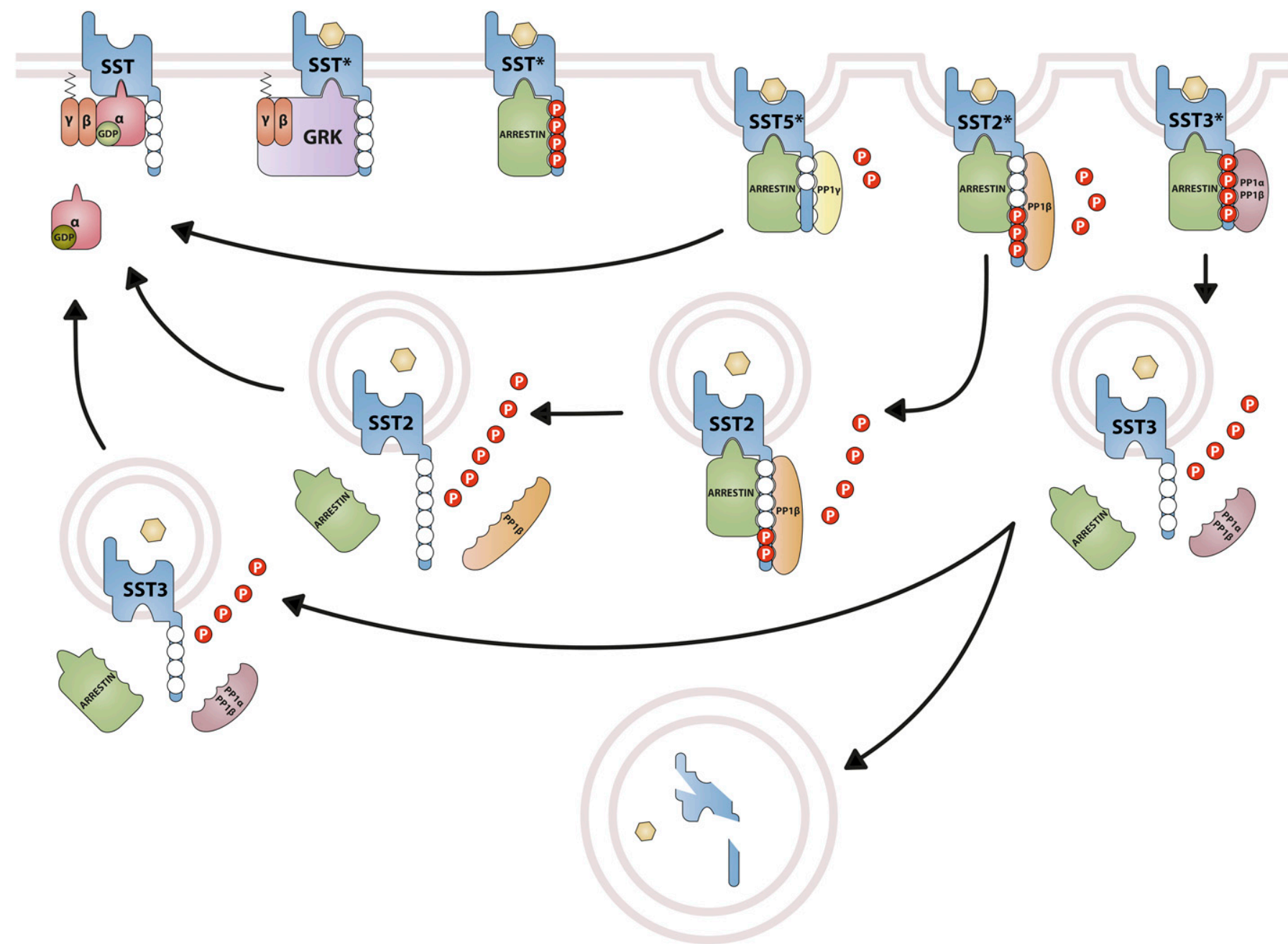

Fig. 20. Differential trafficking of somatostatin receptors. Agonist activation of SSTs triggers activation of the associated heterotrimeric $\mathrm{G}$ protein that in turn stimulates a second messenger system. Quenching of this signal involves phosphorylation of the receptor by GRKs. Phosphorylation by GRKs increases the affinity for arrestins, which uncouple the receptor from the G protein and target the receptor to clathrincoated pits for internalization. Return to its resting state requires dissociation or degradation of the agonist, dephosphorylation, and dissociation of arrestin. For $\mathrm{SST}_{5}$, the catalytic PP1 $\gamma$ subunit was identified to catalyze S/T dephosphorylation at the plasma membrane within seconds to minutes after agonist removal. $\mathrm{SST}_{5}$ forms unstable complexes with arrestins that are rapidly disrupted. After dephosphorylation, $\mathrm{SST}_{5}$ is either resensitized at the plasma membrane or recycled back through an endosomal pathway. For $\mathrm{SST}_{2}$, the catalytic PP1 $\beta$ subunit was identified to catalyze S/T dephosphorylation. $\mathrm{SST}_{2}$ forms stable complexes with arrestins that cointernalize into the same endocytic vesicles. This dephosphorylation process is initiated at the plasma membrane and continues along the endosomal pathway. PP1 $\beta$-mediated dephosphorylation promotes dissociation of arrestins and, hence, facilitates quenching of arrestin-dependent signaling. Subsequently, $\mathrm{SST}_{2}$ is recycled back through an endosomal pathway to the plasma membrane. For $\mathrm{SST}_{3}$, the catalytic PP1 $\alpha / \beta$ subunits were identified to catalyze $\mathrm{S} / \mathrm{T}$ dephosphorylation at the plasma membrane within seconds to minutes after agonist removal. $\mathrm{SST}_{3}$ forms unstable complexes with arrestins that are rapidly disrupted. After dephosphorylation, $\mathrm{SST}_{3}$ is either subject to lysosomal degradation or recycled back to the plasma membrane through an endosomal pathway.

Kumar, 2011). However, although it has been suggested that $\mathrm{SST}_{2}-\mathrm{SST}_{5}$ interaction could elicit relevant functional consequences in the response to SRIF analogs in acromegaly, the precise biologic and physiologic importance of these mechanisms in vivo is still a matter of debate (Grant et al., 2008). Interestingly, the truncated human $\mathrm{SST}_{5} \mathrm{TMD} 4$ variant disrupts normal $\mathrm{SST}_{2}$ homodimerization, whereas it does not interfere with homodimerization of its full-length $\mathrm{SST}_{5}$ counterpart. Consequently, $\mathrm{SST}_{5} \mathrm{TMD} 4$ only, and distinctly, reduced functional responses of $\mathrm{SST}_{2}$ to SRIF, but not that of canonical $\mathrm{SST}_{5}$, which conveys key functional consequences, as the variant may disrupt the inhibitory capacity of $\mathrm{SST}_{2}$ (Durán-Prado et al., 2012b). Indeed, a comparable situation occurs for truncated pig $\mathrm{SST}_{5}$ variants (Durán-Prado et al., 2012a). Heterodimerization of $\mathrm{SST}_{5}$ with a GPCR from a different family, the dopamine receptor $\mathrm{D} 2\left(\mathrm{D}_{2}\right.$ receptor), has also been reported (Rocheville et al., 2000a), and its potential pharmacological consequences in CNS are being explored (Szafran et al., 2012; Szafran-Pilch et al., 2017). SST $_{5}$ formation of heteromers seems to be promiscuous as it has also shown to interact with the ghrelin receptor GHS-R1a, in a context enabling a fine, coordinated regulation of glucose-stimulated insulin secretion by SRIF and ghrelin (Park et al., 2012). In fact, $\mathrm{SST}_{5}$ may even interact physically and functionally with another receptor class, the tyrosine kinase receptors (Kumar, 2011). 


\section{F. Somatostatin Receptor 5 Anatomic Framework}

By using rabbit mAb UMB-4, $\mathrm{SST}_{5}$ was detected both at the plasma membrane and in the cytoplasm of distinct cell populations of different normal human tissues such as GH- and ACTH-producing cells of the anterior pituitary, acinar cells, and striated ducts of the parotid glands, $\mathrm{C}$ cells of the thyroid, neuroendocrine- and enterochromaffin-like cells of the GI mucosa, insulin- and glucagon-secreting cells of the pancreas, cells in the reticular zone of the adrenal cortex and in adrenal medulla, glomerular endothelial cells and tubules of the kidney, luteinized granulosa cells of the ovary, luminal parts of testicular tubuli, lymphocytes in the germinal centers of lymph follicles, alveolar macrophages of the lung, singular cells scattered throughout the stroma of various organs, and single cells observed occasionally in the liver, most probably also representing macrophages (Fig. 21) (Lupp et al., 2011; Unger et al., 2012; Stollberg et al., 2016). Very limited expression of $\mathrm{SST}_{5}$ was detected in the brain of rodents, particularly in specific nuclei in the basal forebrain (Stroh et al., 1999).

Regarding neoplastic tissues, $\mathrm{SST}_{5}$ was observed at a high intensity of expression in all somatotroph and in most of corticotroph adenomas, whereas in gonadotroph and nonfunctioning pituitary adenomas $\mathrm{SST}_{5}$ expression was low (Fig. 21) (Lupp et al., 2011; Gabalec et al., 2015; Lee et al., 2015). Furthermore, the receptor was detected in $38 \%-57 \%$ of medullary and in most of $(>75 \%)$ papillary and follicular thyroid carcinomas (Pazaitou-Panayiotou et al., 2012; Atkinson et al., 2013; Woelfl et al., 2014; Herac et al., 2016). To a variable extent $\mathrm{SST}_{5}$ was additionally detected in pheochromocytomas and paragangliomas (Lupp et al., 2011; Elston et al., 2015) and in functioning and nonfunctioning adrenocortical adenomas (Pisarek et al., 2011). Presence of $\mathrm{SST}_{5}$ was also noticed in lymphomas (Stollberg et al., 2016; Ruuska et al., 2018). Most notably and after $\mathrm{SST}_{2}, \mathrm{SST}_{5}$ represents the second most common SST subtype expressed in gastroenteropancreatic neuroendocrine neoplasms. $\mathrm{SST}_{5}$ was detected in $62 \%-93 \%$ of tumors overall (Lupp et al., 2011; Kaemmerer et al., 2015b; Qian et al., 2016; Song et al., 2016; Wang et al., 2017), with less frequent expression in pancreatic than in GI tumors, and higher expression rates in functioning than in nonfunctioning tumors (Song et al., 2016). Additionally, tumor grade correlates negatively with receptor abundance (Song et al., 2016; Wang et al., 2017), and, hence, a positive association with patient outcomes has been demonstrated for $\mathrm{SST}_{5}$ (Song et al., 2016; Wang et al., 2017). However, $\mathrm{SST}_{5}$ overexpression is also associated with vascular and nerve invasion and thus enhanced aggressiveness (Herrera-Martinez et al., 2017a). In some studies a positive correlation between $\mathrm{SST}_{5}$ expression and SST-based imaging was shown (Diakatou et al., 2015). Furthermore, $\mathrm{SST}_{5}$ was detected in $31 \%-45 \%$ of bronchopulmonary neoplasms (Kaemmerer et al., 2015a; Lapa et al., 2016) and occasionally also in other tumors with neuroendocrine differentiation (Mizutani et al., 2012). $\mathrm{SST}_{5}$ was observed in $15 \%-$ $47 \%$ of GIST, and $\mathrm{SST}_{2}$ and/or $\mathrm{SST}_{5}$ immunoreactivity was associated with increased recurrence-free survival (Arne et al., 2013; Zhao et al., 2014). $\mathrm{SST}_{5}$ was detected in $39 \%-70 \%$ of colorectal cancers, and expression was higher in well to moderately differentiated tumors than in poorly differentiated ones, with a positive correlation with favorable patient outcomes (Qiu et al., 2006; Evangelou et al., 2012). $\mathrm{SST}_{5}$ was also detected in most breast, cervical, ovarian, and prostate carcinomas (Lupp et al., 2011), as well as in $45 \%$ of Merkel cell tumors (Gardair et al., 2015).

\section{G. Somatostatin Receptor 5 Function}

The main physiologic functions of $\mathrm{SST}_{5}$ relate to control of pituitary and pancreatic endocrine secretions (Møller et al., 2003; Olias et al., 2004). $\mathrm{SST}_{5}$ abundance
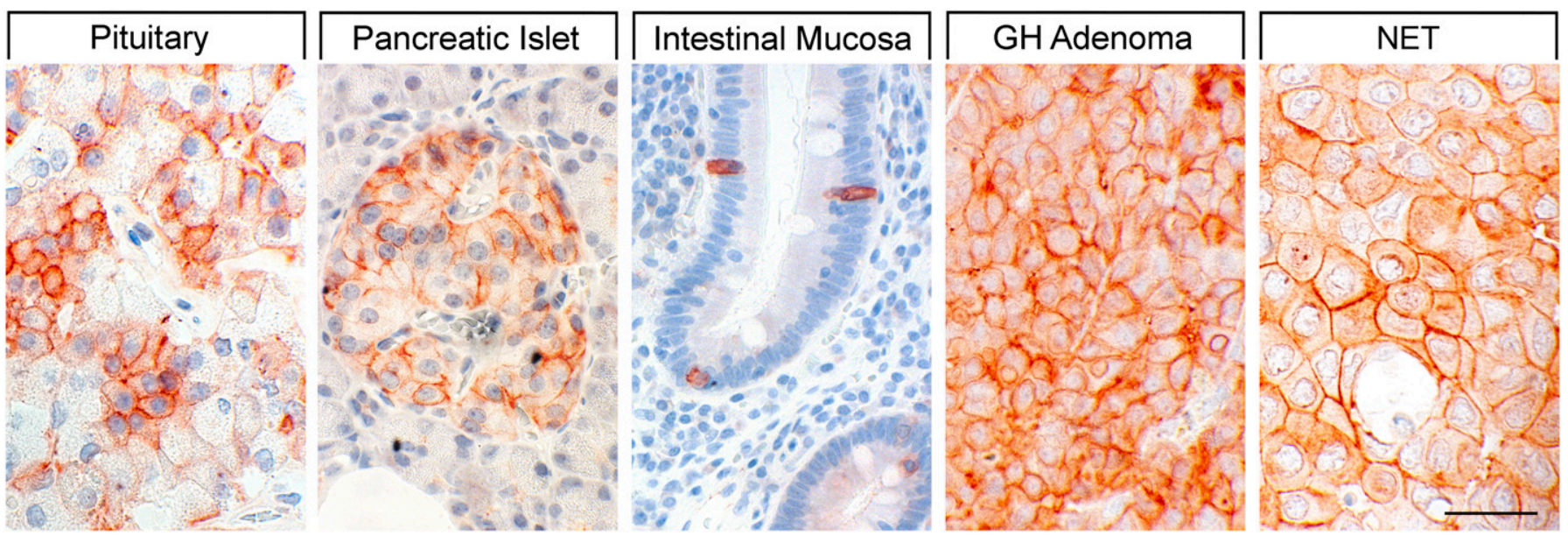

Fig. 21. $\mathrm{SST}_{5}$ expression pattern in human normal and neoplastic tissues. Immunohistochemistry (red-brown color), counterstaining with hematoxylin; primary antibody: UMB-4; scale bar, $50 \mu \mathrm{m}$. $\mathrm{SST}_{5}$ displays a predominant membranous expression. 
in pituitary explains its relevant role in the SRIFmediated inhibition of GH secretion from somatotrophs, and its capacity to inhibit ACTH from corticotrophs and TSH from thyrotrophs (Kumar et al., 1997; Ren et al., 2003; Ben-Shlomo and Melmed, 2010). SST 5 constitutive activity may also contribute to these actions (BenShlomo and Melmed, 2010). Conversely, $\mathrm{SST}_{5}$ does not seem to participate relevantly in the physiologic control of prolactin release, although it can inhibit its secretion in prolactinomas; likewise, there is no evidence that $\mathrm{SST}_{5}$ contributes to regulate gonadotroph function (Møller et al., 2003; Olias et al., 2004; Ben-Shlomo and Melmed, 2010).

In the human endocrine pancreas, $\mathrm{SST}_{5}$ plays an important role in conveying the inhibitory actions of SRIF on glucose-stimulated insulin release, although there is also evidence for a role of $\mathrm{SST}_{2}$ (Zambre et al., 1999; Braun, 2014). Conversely, in rodent $\beta$-cells, $\mathrm{SST}_{5}$ is the most abundant and the predominant inhibitory receptor for glucose-induced insulin secretion, and also appears to be involved in $\beta$-cell development (Strowski et al., 2003; Strowski and Blake, 2008; Braun, 2014). Indeed, altered glucose and insulin regulation is the most prominent phenotype of $\mathrm{SST}_{5} \mathrm{KO}$ mice, which are otherwise devoid of overt pathologic symptoms (Strowski et al., 2003; Ramirez et al., 2004; Wang et al., 2005). $\mathrm{SST}_{5}$ may also contribute to inhibit glucagon secretion from $\alpha$-cells, primarily controlled by $\mathrm{SST}_{2}$ (Braun, 2014).

Presence and functional roles of $\mathrm{SST}_{5}$ in the CNS are relatively limited compared with other SSTs. In the brain, $\mathrm{SST}_{5}$ activation may inhibit stress-related stimulation of hypothalamic CRF and pituitary ACTH release (Stengel and Taché, 2017). $\mathrm{SST}_{5}$ may contribute to regulate sympathetic responses; likewise, $\mathrm{SST}_{5}$ may mediate gastric emptying through activation of vagal cholinergic pathways (possibly with the contribution of other receptors), as supported by its high expression in the dorsal motor nucleus of the vagus nerve (Martinez et al., 2000; Stengel et al., 2013; Stengel and Taché, 2017). Evidence for SST $_{5}$ functions outside its endocrine and CNS actions is limited. $\mathrm{SST}_{5}$ is present in the rat retina, where its activation protects from AMPAinduced neurotoxicity (Kiagiadaki et al., 2010). $\mathrm{SST}_{5}$ is also present in cochlea, but its role and relevance are not yet known (Radojevic and Bodmer, 2014). In the reproductive tract, $\mathrm{SST}_{5}$ is present in Sertoli cells, where its expression is developmentally regulated (Riaz et al., 2013). In the vascular system, $\mathrm{SST}_{5}$ is present in smooth muscle cells of the human and mouse aorta, where it is coexpressed with truncated $\mathrm{SST}_{5} \mathrm{TMD}_{4}$ and $\mathrm{SST}_{2}$ and GHS-R1a to mediate protective actions of CST (DuránPrado et al., 2013). The presence of $\mathrm{SST}_{5}$, either as mRNA or protein, has been described in a wide range of disorders, especially in tumors, where its precise role and potential value remain to be established (Møller et al., 2003; Barbieri et al., 2013). Of particular interest is the presence of $\mathrm{SST}_{5}$ in pituitary adenomas and NETs, for they already represent a valuable pharmacological target for SRIF analog treatment (van der Hoek et al., 2010; Veenstra et al., 2013).

\section{H. Somatostatin Receptor 5 Ligands}

Although pasireotide also exhibits affinity to $\mathrm{SST}_{1}$, $\mathrm{SST}_{2}$, and $\mathrm{SST}_{3}$, it binds with superior affinity to $\mathrm{SST}_{5}$. It also exhibits potent agonistic activity at $\mathrm{SST}_{5}$ and most likely mediates most of its pharmacological actions via $\mathrm{SST}_{5}$ (Petrich et al., 2013). The peptide agonist BIM-23268 displays moderate affinity to all SSTs (Fig. 22; Table 8); however, it appears to be unique among $\mathrm{SST}_{5}$ agonists in that it exhibits full agonistic activity (Shimon et al., 1997a; Petrich et al., 2013).
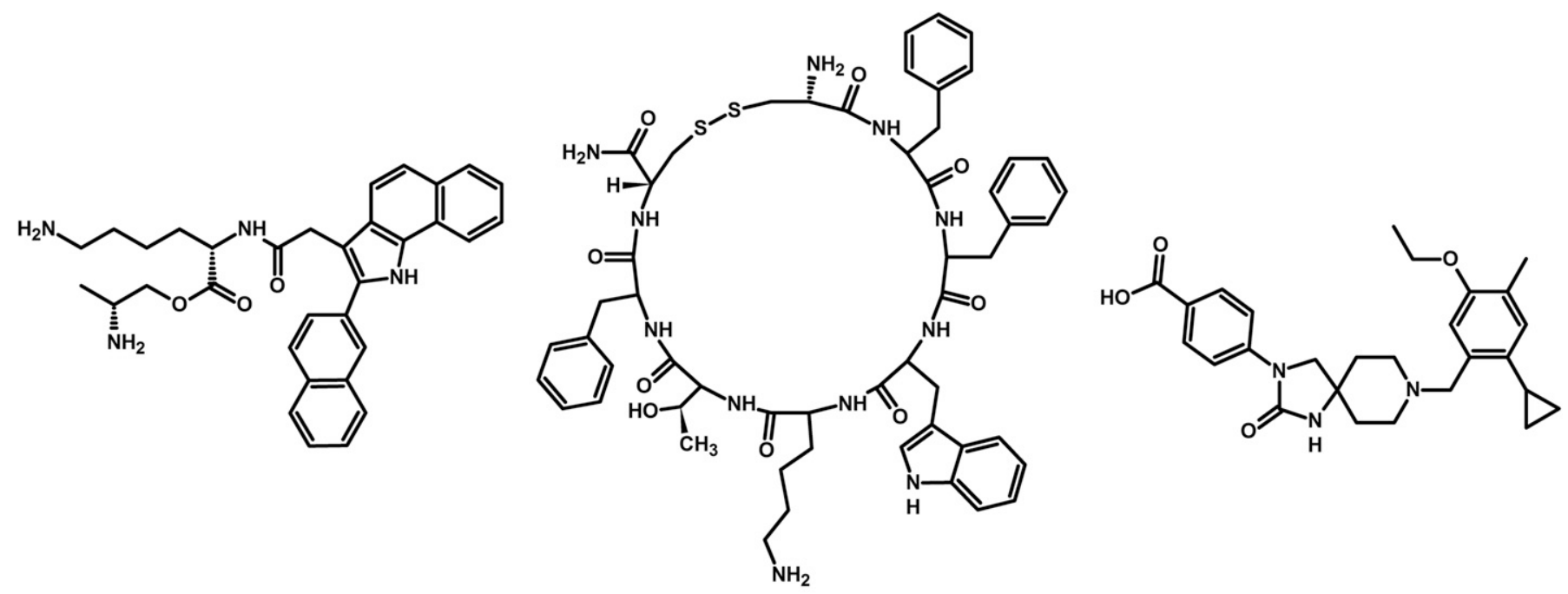

Fig. 22. Structures of synthetic $\mathrm{SST}_{5}$ ligands. L-817,818 and BIM-23268, $\mathrm{SST}_{5}$ agonists; S5A1, $\mathrm{SST}_{5}$ antagonist. 
TABLE 8

Ligand-binding affinities of $\mathrm{SST}_{5}$-selective ligands

\begin{tabular}{lccccl}
\hline & $\mathrm{SST}_{1}$ & $\mathrm{SST}_{2}$ & $\mathrm{SST}_{3}$ & $\mathrm{SST}_{4}$ & $\mathrm{SST}_{5}$ \\
\hline $\mathrm{L} 817 / 818^{a}$ & 3.3 & 52 & 64 & 82 & 0.4 \\
$\mathrm{BIM}^{b} 23268^{b}$ & 18.4 & 15.1 & 61.6 & 16.3 & 0.37 \\
S5A1 $^{c}$ & $>5190$ & $>10,000$ & $>10,000$ & - & 4.87 \\
\hline
\end{tabular}

${ }^{a}$ Data from Rohrer et al. (1998).

${ }^{b}$ Data from Shimon et al. (1997a)

${ }^{c}$ Data from Farb et al. (2017).

Another peptide agonist is BIM-23206, which displays about 50 -fold selectivity for $\mathrm{SST}_{5}$ over $\mathrm{SST}_{2}$ (Ren et al., 2003). L-817,818 is a moderate selective nonpeptidyl agonist (Fig. 22; Table 8) (Rohrer et al., 1998). However, it displays only partial agonistic activity at $\mathrm{SST}_{5}$ (Petrich et al., 2013). A series of benzoxazole piperidines was identified as high-affinity $\mathrm{SST}_{5}$ antagonists with virtual absence of binding to other SSTs (Martin et al., 2009). More recently, several $\mathrm{SST}_{5}$-selective antagonists such as S5A1 were evaluated as potential treatments for diabetes mellitus (Fig. 22). S5A1 displays a subnanomolar affinity for $\mathrm{SST}_{5}$ (Table 8) (Farb et al., 2017).

\section{Multireceptor Somatotropin-Release Inhibitory Factor Analogs}

\section{A. Evolution of Concepts}

The rationale for desired characteristics of therapeutically useful SRIF analogs has evolved as knowledge of receptor subtypes and their interactions has become available. Following the discovery of SRIF as the hypothalamic factor responsible for suppression of GH secretion (Brazeau et al., 1973), it became apparent that it was involved in multiple additional physiologic functions (Reichlin, 1983a,b). Due to the rapid degradation and clearance of the native SRIF peptide, efforts were focused on creating analogs with increased metabolic stability that would be useful for treating conditions of excess GH secretion, most notably acromegaly. As the structure of native SST was modified, differences were observed in the ratio of GH-suppressing activity versus other actions, in particular the suppression of insulin, which was considered a potential problem for therapeutic application (Grant et al., 1976; Brown et al., 1977; Meyers et al., 1977; Coy et al., 1978). Through screening in rodents, analogs were identified with potent GH-suppressing activity with acceptably low insulin-suppressing activity (Bauer et al., 1982; Heiman et al., 1987), including the two SRIF analogs still most widely used clinically for treatment of acromegaly and NETs, octreotide (Sandostatin) and lanreotide (Somatuline) (Fig. 23; Table 9).

The reason that the $\mathrm{GH}$ and insulin-suppressing activities could be dissociated by structural modification of SRIF was later explained with the identification of five distinct SSTs (Reisine and Bell, 1995). With the realization that there were multiple receptor subtypes, the concept emerged that different subtypes controlled different, specific biologic activities, and that functional selectivity might be achieved by analogs with preference or, ideally, selectivity for one specific receptor subtype. The task then became determining which receptor subtype controlled which specific function. This was approached by creating libraries of SRIF analogs that were fully characterized for their preferences or selectivity for the different receptor subtypes. By screening panels of analogs with varying selectivities in different biologic models, it was hoped that the receptor subtype responsible for a particular biologic action could be identified, and could thus become the basis for therapeutically useful compounds targeting a specific function.

One of the first questions to be addressed by this approach was the involvement of the SSTs in suppressing GH secretion in humans. Although the two earlier discovered analogs, octreotide and lanreotide, are the most widely used medical therapy for acromegaly, they do not normalize GH and IGF-1 levels in a significant percentage of patients with acromegaly. A recent metaanalysis of clinical studies with sustained release octreotide and lanreotide indicated normalization of

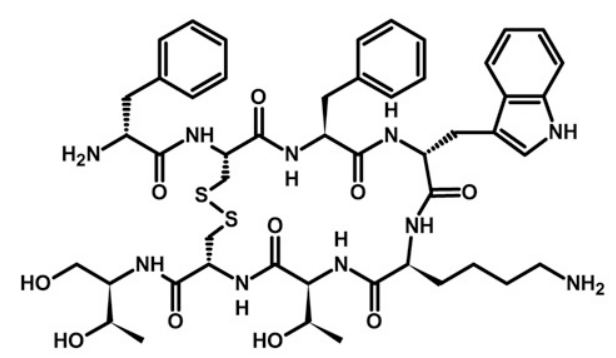

Octreotide

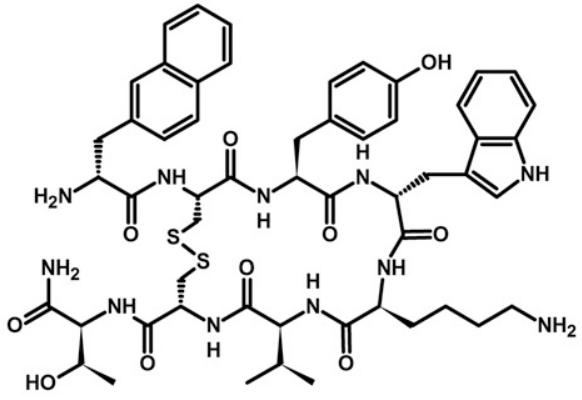

Lanreotide

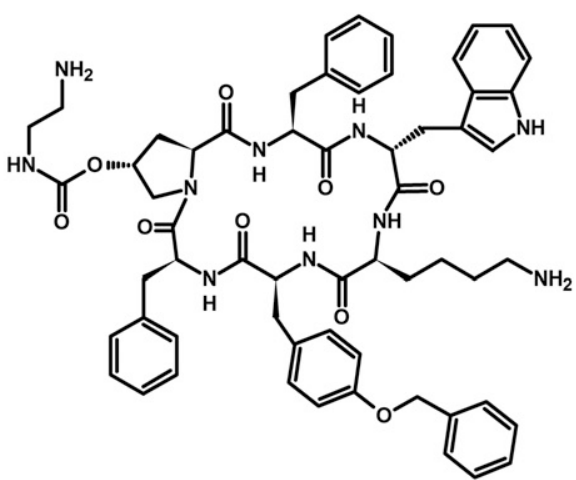

Pasireotide

Fig. 23. Structures of SRIF ligands currently used in clinical practice. 
TABLE 9

Ligand-binding affinities for approved and investigational SRIF ligands

\begin{tabular}{lclccl}
\hline & $\mathrm{SST}_{1}$ & $\mathrm{SST}_{2}$ & $\mathrm{SST}_{3}$ & $\mathrm{SST}_{4}$ & $\mathrm{SST}_{5}$ \\
\hline Octreotide $^{a}$ & $>1000$ & 0.4 & 4.4 & $>1000$ & 5.6 \\
Lanreotide $^{b}$ & 2129 & 0.75 & 98 & 1826 & 5.2 \\
Pasireotide $^{c}$ & 9.3 & 1 & 1.5 & $>100$ & 0.16 \\
Veldoreotide $^{d}$ & $>1000$ & 3 & $>100$ & 7 & 6 \\
\hline
\end{tabular}

${ }^{a}$ Data from Reisine and Bell (1995), Patel (1999).

${ }^{b}$ Data from Shimon et al. (1997b), Zatelli et al. (2001).

${ }^{c}$ Data from Bruns et al. (2002).

${ }^{d}$ Data from Afargan et al. (2001).

GH and IGF-1 in 56\% and 55\%, respectively, of treated patients with acromegaly (Carmichael et al., 2014); however, most of the included studies did not use the current normalization criteria of $\mathrm{GH}<1 \%$ and did not consider composite GH and IGF-1 normalization (Gadelha et al., 2017). The PRIMARYS study, which assessed treatment-naive, unselected acromegaly patients, found that only $30 \%$ of patients were fully controlled by these clinically approved somatostatin receptor ligands (Caron et al., 2014).

With characterization of their interaction with the five SSTs, both lanreotide and octreotide were found to have potent subnanomolar affinity for the $\mathrm{SST}_{2}$ subtype, with moderate affinity for $\mathrm{SST}_{5}$ (Table 9). Lanreotide and octreotide also have moderate affinity for $\mathrm{SST}_{5}$ receptors, but that does not translate functionally (Siehler et al., 1998; Siehler and Hoyer, 1999a,b). Not surprisingly, studies using subtype-selective analogs confirmed that the $\mathrm{SST}_{2}$ subtype was indeed responsible for suppression of $\mathrm{GH}$ in the rat, the species used for biologic optimization of lanreotide and octreotide (Raynor et al., 1993b; Briard et al., 1997). In contrast, however, when ligand panels were tested in cultures of human fetal pituitaries, it was found that activation of both $\mathrm{SST}_{2}$, as well as the $\mathrm{SST}_{5}$ subtype, induced suppression of $\mathrm{GH}$ secretion, and that activation of either of the two receptors was equally efficacious (Shimon et al., 1997a). Furthermore, when the two receptors were activated together, GH-suppressing activity was significantly enhanced, well beyond that observed with the clinically used $\mathrm{SST}_{2}$-selective analogs, lanreotide and octreotide (Shimon et al., 1997b). Considering that native SRIF potently interacts with all five receptor subtypes, it is reasonable to assume that the enhanced suppression of GH induced by dual receptor activation is the normal mechanism employed by SRIF in the physiologic control of GH. These observations also suggest that the lack of potent $\mathrm{SST}_{5}$ activity in the clinically used analogs may be the reason for the lack of full control of GH and IGF-1 in a significant number of patients with acromegaly.

The need for greater $\mathrm{SST}_{5}$ activation for a more complete suppression of GH was confirmed in studies of cultures of pituitary adenoma cells from patients with acromegaly classified as only partially responsive to SRIF analog therapy (Jaquet et al., 2000). In keeping with clinical experience, the $\mathrm{SST}_{2}$-preferring analog, octreotide, induced only a partial suppression of GH in the cultured adenoma cells. Treatment with more potent $\mathrm{SST}_{2}$ - or $\mathrm{SST}_{5}$-selective analogs produced somewhat greater suppression. However, combined $\mathrm{SST}_{2^{-}}$ and $\mathrm{SST}_{5}$-selective analog treatment to activate both receptor subtypes produced greatly enhanced suppression of $\mathrm{GH}$, thus supporting the concept that greater activity at $\mathrm{SST}_{5}$ is required to normalize $\mathrm{GH}$ in a greater percentage of acromegaly patients than is achieved with lanreotide and octreotide (Jaquet et al., 2000).

Based on these observations, the first multisubtypeselective analog, BIM-23244, was produced with $2 \times$ greater activity at $\mathrm{SST}_{2}$, and $20 \times$ greater activity at $\mathrm{SST}_{5}$, as compared with octreotide and lanreotide (Saveanu et al., 2001). To test the feasibility of biselective receptor activity in a single compound, GH-secreting tumors were collected from 10 patients with acromegaly that were clinically classified as either fully or only partially responsive to current SRIF analog therapy. Cultured adenoma cells from the fully responsive patients responded equally to treatment with either octreotide or the $\mathrm{SST}_{2+5}$ biselective analog, BIM23244; however, octreotide produced only a partial response in cells from partially responsive patients, whereas BIM-23244 produced a greatly enhanced response, similar to that observed in the adenoma cells from fully responsive patients (Saveanu et al., 2001). These results substantiate the benefit from dual activation of both receptor subtypes and validate the concept that both activities can be presented in a single compound and retain the enhanced biologic action.

These observations opened the possibility of receptor subtype interactions in other tissues and biologic functions. Studies of gene receptor expression had already demonstrated that various tissues contained multiple SSTs, and that expression of these subtypes could change depending on the physiologic conditions or as a result of pathology (Bruno et al., 1993; Patel et al., 1996; Reubi et al., 1997; Kimura et al., 2001). An example of the latter is the shift in SRIF suppression of prolactin secretion from $\mathrm{SST}_{2}$-mediated in normal human pituitary cells to $\mathrm{SST}_{5}$-mediated in cells from human prolactinomas (Saveanu et al., 2001). In addition, the expression of SSTs can change temporally during the course of a specific physiologic or pathologic process. Khare et al. (1999) demonstrated that, following damage to the endothelial lining of the aorta, the pattern of SSTs expressed changed over the subsequent days as the various stages of repair occurred. Observations such as these raised the possibility that multireceptor-interacting ligands could not only produce enhanced actions, but by targeting selected combinations of receptor subtypes, might also provide selectivity for specific physiologic or pathologic states.

\section{B. Potential Mechanisms}

The mechanism by which SRIF action is enhanced by activating a combination of receptors remains uncertain. 
The simplest possibilities would be activation of two separate transduction pathways that have a common biologic endpoint, or greater activation of a single transduction pathway shared by two or more receptors. However, another possibility with a growing body of evidence is that various receptors can physically interact to form homo- or heterodimers, with resulting changes in activity. This phenomenon was first reported to occur for members of the opioid receptor family, which are structurally related to SSTs. Jordan and Devi (1999) reported that heterodimers formed by the $\kappa$ and $\delta$ opioid receptors resulted in unique ligand-binding properties and, when activated, a potentiation of signal transduction. Formation of both homo- and heterodimers has subsequently been reported to occur among the SSTs as well, including $\mathrm{SST}_{2}$ and $\mathrm{SST}_{5}$. Dimerization of receptors with resulting alterations in ligand interactions suggests a unique opportunity to develop analogs that recognize only the specific homodimer of one receptor or the heterodimer of multiple receptor types, to achieve the highest level of functional specificity and efficacy.

The approach of using subtype-selective analogs to determine which SSTs are involved in a particular biologic action has continued using different models. In some cases, single-receptor subtypes do appear to be the dominant mechanism. Examples include suppression of insulin mediated by $\mathrm{SST}_{5}$ on human $\beta$-cells (Zambre et al., 1999), glucagon by $\mathrm{SST}_{2}$ on $\alpha$-cells (Strowski et al., 2000), and vessel out-sprouting by $\mathrm{SST}_{1}$ from cultured human placental vein explants, a model of angiogenesis (Bocci et al., 2007). As direct suppression of insulin is mediated by $\mathrm{SST}_{5}$, the early observation that certain analogs could suppress $\mathrm{GH}$ while having minimal effect on insulin explains the modest amount of $\mathrm{SST}_{5}$ activity of the analogs selected for clinical use, lanreotide and octreotide.

As studies continued examining combinations of receptor subtype activation for potential enhancement, it was observed that receptor subtype interactions can also be antagonistic. Testing the effect of $\mathrm{SST}_{2}$ and 5 activation on proliferation of thyroid medullary carcinoma cells, it was observed that $\mathrm{SST}_{2}$-selective analogs induce dose-related inhibition of proliferation, whereas $\mathrm{SST}_{5}$-selective analogs cause an increase (Zatelli et al., 2001). When combined, increasing concentrations of the $\mathrm{SST}_{5}$-selctive analog prevents the suppression of proliferation by the $\mathrm{SST}_{2}$-selective ana$\log$, in a dose-related manner, such that at equimolar concentrations the effect of both is neutralized. In this instance, coactivation of $\mathrm{SST}_{2}$ and 5 results in an antagonistic interaction, as opposed to the enhanced biologic effect observed by coactivation of these same receptors on GH secretion. These results indicate that the biologic consequence of receptor subtype interaction is not only a function of the receptors involved, but also the specific cell type or tissue in which they are expressed.
A partial explanation of the antagonistic effects of certain SSTs may be the inactivation of one or both receptors as a result of conformational changes following heterodimerization. In studies examining expression and function of $\mathrm{SST}_{2}$ and $\mathrm{SST}_{3}$ receptors expressed individually, Pfeiffer et al. (2001) demonstrated homodimerization of both subtypes and induction of specific transduction mechanisms when activated. When coexpressed, however, heterodimerization between the two subtypes was observed with the consequence of retained activation and signal transduction with $\mathrm{SST}_{2}$-selective ligands, but a complete loss of activation and signaling with $\mathrm{SST}_{3}$-selective ligands. These results clearly illustrate the exponential increase in complexity in moving from the initial targeting of individual receptor subtypes to affect a specific function to the targeting of various subtype combinations.

Although complex, the concept of targeting the interaction between multiple receptor subtypes remains attractive for enhanced efficacy; however, due to the widespread distribution of SSTs in different tissues, the original concern still remains that a metabolically stable compound able to activate multiple receptor subtypes could induce unwanted side effects. As an example, although the previously described biselective analog, BIM-23244, with selective, potent interaction with both $\mathrm{SST}_{2}$ and ${ }_{5}$, yields superior GH suppression, and potentially greater therapeutic benefit for a wider range of patients suffering from acromegaly, the direct suppression of insulin by $\mathrm{SST}_{5}$ raises the potential for unwanted pancreatic side effects. To examine the consequence of activating $\mathrm{SST}_{2}$ versus $\mathrm{SST}_{5}$, a study was conducted in healthy volunteers in which a SRIF analog with potent, selective $\mathrm{SST}_{2}$ activity was compared with a potent $\mathrm{SST}_{5}$ analog. Infusion of the $\mathrm{SST}_{2}$ analog resulted in a doserelated decrease in glucagon and insulin, but without effect on glucose levels. In addition, administration of an amino acid challenge during the $\mathrm{SST}_{2}$ analog infusion resulted in an appropriate insulin response, again maintaining normal glycemic control. Infusion of the $\mathrm{SST}_{5}$ analog, however, resulted in a dramatic suppression of insulin secretion, with resulting hyperglycemia, and failure of the $\beta$-cells to respond to the amino acid challenge. As a result of this potentially severe side effect mediated by $\mathrm{SST}_{5}$, BIM-23244 was not further developed, and design of subsequent analogs aimed for modest interaction with $\mathrm{SST}_{5}$. These results illustrate that the original concern of potential side effects when activating multiple receptors is a legitimate consideration.

\section{Pasireotide}

A different concept from teasing out the involvement of specific SSTs in specific functions with the idea of creating subtype-specific, and therefore functionspecific analogs, was to create clinically useful receptor ligands that are metabolically stable, but mimic the ability of native SRIF to interact with all five receptor 
subtypes. A theoretical advantage of this approach is that, assuming the activities are correctly proportioned, it should be possible to take advantage of receptor subtype interactions to produce different or enhanced responses from those achieved by activation of a single receptor subtype. The risk is that receptors mediating unwanted effects could also be activated. This approach was exemplified by development of the pan-receptorspecific ligand, pasireotide (also known as Signifor), a cyclohexapeptide with reasonably high affinity for $\mathrm{SST}_{1}, 2$, and ${ }_{3}$, no interaction with $\mathrm{SST}_{4}$, but exceptionally high, subnanomolar affinity for $\mathrm{SST}_{5}$ (Table 9) (Bruns et al., 2002). In studies in rats, pasireotide produced comparable suppression of GH secretion as octreotide, but with much greater duration owing to a significantly greater circulating $t_{1 / 2}$. In long-term infusion studies, pasireotide was considerably more effective in lowering IGF-1 than octreotide (Bruns et al., 2002). Because IGF-1 production is regulated by both GH-dependent and independent mechanisms, the enhanced action of pasireotide may be the result of interaction with receptor subtypes other than $\mathrm{SST}_{2}$, which is the primary mediator of octreotide action.

In a clinical trial directly comparing sustained release formulations of pasireotide and octreotide in a large cohort of randomly allocated, medically naive patients with acromegaly, pasireotide was found to control GH and IGF-1 in a greater percentage (31.3\% pasireotide treatment versus $19.2 \%$ octreotide treatment) of subjects (Colao et al., 2014). Furthermore, in patients resistant to octreotide or lanreotide treatment, sustained-release pasireotide was found to achieve control in 15\%-20\% of subjects, depending on dosage (Gadelha et al., 2014). These results may be explained by the study of Gatto et al. (2017), in which pasireotide was compared with octreotide for their ability to suppress GH secretion from in vitro cultures of tumor cells from acromegalic patients. Overall, the two were equivalent; however, pasireotide was found to be more effective than octreotide in a subgroup of the cultures from tumors with a comparatively lower expression of $\mathrm{SST}_{2}$ and $\mathrm{SST}_{2} / \mathrm{SST}_{5}$ ratio (Gatto et al., 2017). In keeping with the high activity of pasireotide at $\mathrm{SST}_{5}$, however, a significantly higher percentage of patients experienced hyperglycemiarelated adverse events in the two clinical studies with pasireotide treatment as compared with octreotide or lanreotide treatment (Colao et al., 2014; Gadelha et al., 2014). The potential of pasireotide has also been investigated in Cushing's disease.

\section{Dopastatin}

Extending further the concept of multireceptor SRIF ligands is the creation of compounds that interact with SSTs as well as receptors outside the SST family. This concept originally derived from clinical studies indicating that combined treatment of acromegalic patients with both SRIF and dopamine analogs resulted in greater control of GH and IGF-1 than the use of either agent alone (Fløgstad et al., 1994; Minniti et al., 1997; Marzullo et al., 1999; Li et al., 2000). This generated the idea to create chimeric compounds that contain structural elements of both SRIF and dopamine, and that retain the ability to bind to receptors of both. In initial studies in primary cultures of human GH-secreting adenoma cells, it was observed that whereas both pure SRIF and pure dopamine analogs were able to induce dose-related suppression of GH secretion, the combination of the two individual agents produced no greater suppression of GH than the SRIF analog alone. However, when both activities were combined in a single, chimeric compound, able to interact with both the $\mathrm{SST}_{2}$ and $\mathrm{D}_{2}$ receptor, significantly enhanced potency as well as efficacy is observed (Saveanu et al., 2002; Jaquet et al., 2005). The mechanism for this enhanced activity remains unknown; however, one possible explanation is the reported demonstration of heterodimer formation between both $\mathrm{SST}_{2}$ and ${ }_{5}$, and $\mathrm{D}_{2}$ receptor (Rocheville et al., 2000a).

Further refinement of the ratio of activities in the chimeric compound resulted in the production of BIM23A760, which binds to $\mathrm{SST}_{2}(0.03 \mathrm{nM}), \mathrm{SST}_{5}(42 \mathrm{nM})$, and $\mathrm{D}_{2}$ receptor $(16 \mathrm{nM})$ (Jaquet et al., 2005). The modest affinity at $\mathrm{SST}_{5}$ is intentional to avoid potential pancreatic effects, as previously discussed. The lack of glycemic side effects was confirmed in normal cynomolgous monkeys in which administration of BIM-23A760 produced potent, dose-related suppression of GH and IGF-1, but had no effect on either insulin secretion or circulating glucose. In addition to suppression of secretion, BIM-23A760 has been demonstrated to have potent antiproliferative effects, producing dose-related suppression of cultured primary human nonfunctioning pituitary adenoma cells (Florio et al., 2008) and somatotropinoma cells (Ibáñez-Costa et al., 2017a). Furthermore, complete arrest of spontaneously developing, aggressive, nonfunctioning pituitary adenomas was observed in vivo in proopiomelanocortin $\mathrm{KO}$ mice, an effect not observed with pure SRIF or dopamine analogs, either alone or in combination. These results suggest that chimeric compounds, such as BIM-23A760, may be effective in controlling pituitary diseases of hypersecretion, as well as impacting the growth of the underlying causative tumor.

Clinical development of BIM-23A760 was initiated and produced a clean safety profile in phase I and a significant demonstration of efficacy in a phase IIa, single-dose study in acromegalic subjects. Unfortunately, with repeated administration in humans, a long-acting, highly potent dopaminergic metabolite was produced that gradually accumulated and diminished the action of the parent compound, BIM-23A760. Subsequently, after further structure-activity studies, a second generation chimera, BIM-23B065, was produced with significantly greater potency and efficacy 
than BIM-23A760, as demonstrated by suppression of GH secretion from primary cultures of human GH-secreting adenoma cells from patients classified as both fully and only partially responsive to the currently used SRIF analogs, octreotide and lanreotide, and without formation of an interfering metabolite. BIM23B065 is currently in early development.

From the initial discovery of SRIF and its receptor subtypes, the rationale for therapeutically useful analogs has evolved from targeting a single-receptor subtype to control a specific function and to limit potential side effects, to targeting specific combinations of receptors to induce enhanced effects for a specific function. Although progress has been made in terms of elucidating specific, disease-related combinations of receptors that act together, the initial concern of inducing side effects through activation of receptors in nontargeted tissues has been demonstrated to be a legitimate consideration. Future analogs that can specifically interact with targets, such as the unique binding pockets of homoand heterodimers formed from the SST subtypes, as well as other receptor families, may yet achieve the full potency, selectivity, and safety potential envisioned for SRIF analogs.

\section{Somatotropin-Release Inhibitory Factor Analogs in Current Clinical Practice}

Hypothalamic SRIF traverses the hypothalamicpituitary portal vessels to impinge on anterior pituitary cells that express multiple SSTs. SRIF analogs show target selectivity for receptor subtype and functional selectivity in regulating $\mathrm{GH}, \mathrm{ACTH}$, and TSH secretion (Shimon et al., 1997b). Somatotroph cells predominantly express $\mathrm{SST}_{2}>\mathrm{SST}_{5} . \mathrm{SST}_{2}$ signals to suppress GH secretion and may also regulate somatotroph tumor growth, whereas $\mathrm{SST}_{5}$ predominantly suppresses corticotroph ACTH release (Table 1). Studies with human GH-secreting tumor cell cultures showed a similar receptor profile and functional response to SRIF analogs (Shimon et al., 1997a). TSH-secreting pituitary adenomas (TSHomas) express $\mathrm{SST}_{2}$ and $\mathrm{SST}_{5}$ (Gatto et al., 2012). Subsequent studies revealed that anterior pituitary SSTs may also signal in a ligand-independent action (BenShlomo et al., 2005; Vlotides et al., 2006). Thus, constitutive SST signaling may regulate ambient pituitary hormone secretion to maintain tonic hormone control in the absence of SRIF. These observations have supported the development of therapeutic molecules targeting different SSTs. SRIF analogs with higher affinity for $\mathrm{SST}_{2}$ are more efficacious for control of $\mathrm{GH}$ hypersecretion in acromegaly or TSH hypersecretion from thyrotropinomas (Melmed, 2003). In contrast, pasireotide, which exhibits an affinity-binding profile more similar to natural SRIF-14 (Weckbecker et al., 2002; Ben-Shlomo et al., 2009a), is particularly suitable for suppressing ACTH in patients with pituitary-dependent Cushing disease.

\section{A. Treatment}

1. Acromegaly. The $\mathrm{SST}_{2}$ subtype is preferentially expressed on somatotroph cell surfaces and regulates GH secretion by suppressing intracellular cAMP levels (Greenman and Melmed, 1994a,b; Shimon et al., 1997b). SRIF analog formulations with high $\mathrm{SST}_{2}$ affinity employed for treating acromegaly, namely octreotide and lanreotide, have proven safe and effective for long-term acromegaly management (Table 10). Octreotide, an octapeptide, inhibits GH secretion with a potency 45 times greater than endogenous SRIF, with minimal suppression of insulin release (Lamberts, 1988). As the molecule is relatively resistant to enzymatic degradation, the in vivo $t_{1 / 2}$ is prolonged (up to 2 hours) after s.c. injection. Lanreotide is a structurally related eight-amino-acid cyclic peptide (Castinetti et al., 2009). Responsiveness to both compounds correlates with $\mathrm{GH}$-secreting adenoma $\mathrm{SST}_{2}$ expression (Casarini et al., 2009). Rebound GH hypersecretion that occurs after SRIF infusion is not apparent after

TABLE 10

Approved and investigational SRIF analogs blocking GH secretion

Data adapted from Melmed (2016).

\begin{tabular}{lll}
\hline \multicolumn{1}{c}{ Agent } & \multicolumn{1}{c}{ Description } & Regulatory Status \\
\hline Lanreotide autogel & Long-acting lanreotide & Available \\
Octreotide LAR & Administered via deep s.c. injection every 4-6 wk & Available \\
Pasireotide LAR & Long-acting octreotide & Administered via i.m. injection every 4 wk \\
& Long-acting pasireotide & Available \\
Octreotide capsules & Administered via i.m. injection every 4 wk & Completed phase 3 \\
& Octreotide encapsulated with transient permeability & \\
enhancer & Administered orally twice daily & In phase II \\
& Octreotide bound in liquid crystal matrix & \\
VAM2029 & Administered via s.c. injection at a frequency not yet & In phase II \\
& determined but likely to be every 4 wk & \\
& Adminalog highly selective for GH suppression & \\
& determined but likely to be every 4 wk & \\
\hline
\end{tabular}


administration of either peptide, offering unique advantages for safe, long-term acromegaly therapy (Lamberts et al., 1996). Pasireotide exhibits a preferential high affinity to $\mathrm{SST}_{5}$ (39-fold higher than octreotide), and also binds to $\mathrm{SST}_{1}, \mathrm{SST}_{2}$, and $\mathrm{SST}_{3}$. Octreotide and pasireotide similarly inhibited free cytosolic calcium and $\mathrm{GH}$ release in vitro, in human somatotropinoma cell cultures, where they also comparably reduced GH mRNA levels and cell viability (Ibanez-Costa et al., 2016). Indeed, using cultures derived from 33 in vitro human pituitary tumors in a head-to-head study, octreotide and pasireotide exhibited equivalent antisecretory efficacy in suppressing GH (Gatto et al., 2017).

a. Effects on biochemical control. Both lanreotide and octreotide exhibit similar clinical efficacy and sideeffect profiles (Murray and Melmed, 2008). When defining disease outcomes, it is apparent that up to $40 \%$ of patients receiving SRIF analogs exhibit discordant $\mathrm{GH}$ and IGF-1 levels. Measuring IGF-1 levels, rather than GH levels, during an oral glucose tolerance test appears to more rigorously reflect disease control (Carmichael et al., 2009). Injectable depot SRIF analog formulations are safe and long-acting and enable maximal biochemical control. Drug levels peak 28 days after injection of sustained release i.m. microsphere preparation of octreotide long-acting release (LAR) (20-30 mg) (Fløgstad et al., 1997; Lancranjan et al., 1999), with concomitant GH levels suppressed for up to 49 days. In an open-label study, $70 \%$ of 151 patients responsive to octreotide showed GH levels suppressed to $<2.5 \mathrm{ng} / \mathrm{ml}$ (Lancranjan et al., 1999). Similarly, GH $<2 \mathrm{ng} / \mathrm{ml}$ and normal IGF-1 levels were achieved in $70 \%$ of 36 patients followed for up to 18 years (Maiza et al., 2007). The water-soluble lanreotide autogel $(60,90$, or $120 \mathrm{mg})$ administered by deep s.c. injection every 28-42 days suppressed GH to $<2.5 \mathrm{ng} / \mathrm{ml}$ in 130 patients at 1 year (Neggers et al., 2015). In a randomized 12-month study, 358 patients received pasireotide LAR (40 mg) or octreotide LAR (20 mg), and biochemical control was achieved in $31 \%$ and $19 \%$ of subjects, respectively (Colao et al., 2014). Among those resistant to maximal doses of octreotide or lanreotide, $15 \%$ and $20 \%$ of resistant patients subsequently achieved control when placed on 40 or $60 \mathrm{mg}$ pasireotide, respectively (Gadelha et al., 2014). Control of GH and IGF-1 levels is more favorable in patients with $\mathrm{GH}$-secreting microadenomas (Ezzat et al., 1992). Octreotide, lanreotide, and pasireotide fall short of maximal treatment goals (i.e., normalized GH and IGF-1) in a large subset of patients. Despite medication adherence rates approaching 90\% (Gurel et al., 2017), a global meta-analysis showed $\sim 55 \%$ control rates for both GH and IGF-1 with SRIF analogs (Carmichael et al., 2014).

b. Effects on disease comorbidities. SRIF analogs used as first-line therapy administered prior to surgery in selected patients may ameliorate preoperative morbidity, including heart failure or respiratory or metabolic disorders, thus enabling safer anesthesia (Colao et al., 2004). Furthermore, preoperative treatment may enhance the success of postoperative outcomes by shrinking large tumor masses prior to debulking procedures (Carlsen et al., 2008; Shen et al., 2010; Giustina et al., 2014; Katznelson et al., 2014). A subset of patients with minimally or noninvasive macroadenomas is most likely to benefit from preoperative therapy (Jacob and Bevan, 2014). In a meta-analysis of 64 reports, SRIF analogs were shown to significantly reduce GH-secreting pituitary tumor size (Giustina et al., 2012). Moreover, a meta-analysis showed a modestly beneficial effect of preoperative SRIF analogs on postoperative biochemical control (Pita-Gutierrez et al., 2013), but subsequent longer-term follow-up has not borne out these results (Fougner et al., 2014). Up to $60 \%-80 \%$ of patients harboring microadenomas, macroadenomas, and locally invasive tumors experience a reduction of pituitary adenoma size (Bevan, 2005; Freda et al., 2005; Giustina et al., 2012), with tumor shrinkage seen by 6 months of therapy initiation (Colao et al., 2016). The magnitude of shrinkage is variable, but some patients respond with $>50 \%$ decrease in tumor mass. Given these observations, the use of preoperative SRIF analogs to improve surgical outcomes has been debated. Although studies have shown that postoperative biochemical control is in fact improved by presurgical SRIF analog treatment (Carlsen et al., 2008; Nunes et al., 2015), the overall evidence is dampened by the short follow-up duration and insufficient prospective evidence.

The beneficial impact of SRIF analogs on acromegaly comorbidities is variable, especially for cardiovascular dysfunction, and is determined by age, disease duration, and degree of biochemical disease control. Clinical benefits of SRIF analogs are achieved both by ameliorating deleterious effects of chronic GH and IGF-1 exposure, as well as likely reversal of fluid retention and swelling. For example, GH-induced epithelial sodium channel-dependent sodium transport actively leads to volume expansion and soft-tissue swelling, effects largely reversed by SRIF analogs (Kamenický et al., 2014). Hypertension, likely arising from chronic vascular damage, is usually not reversible by SRIF analogs. However, with biochemical control, doses of antihypertensive drugs required to normalize blood pressure may be decreased (Annamalai et al., 2013). Structural cardiac abnormalities, including myocardial hypertrophy and heart failure, are improved with biochemical control, especially in younger patients and in those with a shorter disease duration (Annamalai et al., 2013). Features of obstructive sleep apnea are usually improved with SRIF analog therapy (Annamalai et al., 2013), but the disorder may persist despite satisfactory biochemical control. Although joint pain and arthropathy are markedly improved symptomatically by SRIF analogs, structural joint damage and associated arthritis are usually irreversible, despite achievement of 
biochemical control. Headache is particularly responsive to short-acting SRIF analog therapy (Williams et al., 1987; Musolino et al., 1990; Levy et al., 2003; Marina et al., 2015).

As GH is a potent antagonist of insulin action, uncontrolled acromegaly is associated with insulin resistance, hyperglycemia, and eventually diabetes. SRIF analogs exert a dual effect on glucose control. $\mathrm{As} \mathrm{SST}_{5}$ is expressed on the pancreatic $\beta$-cells, SRIF analogs with higher $\mathrm{SST}_{5}$ affinity (pasireotide $>$ octreotide and lanreotide) suppress insulin secretion, leading to hyperglycemia and diabetes. By contrast, the potent $\mathrm{GH}$ suppression achieved by $\mathrm{SST}_{2}$-preferential SRIF analogs (octreotide and lanreotide) leads to enhanced insulin sensitivity and lowering (or normalizing) blood glucose levels.

c. Side effects. As $\mathrm{SST}_{2}$ and $\mathrm{SST}_{5}$ are ubiquitously expressed, especially in the GIT, it is not surprising that several off-target side effects are experienced. Transient abdominal pain, bloating, nausea, and diarrhea are commonly encountered. Asymptomatic gallstones, likely due to suppressed CCK and decreased gallbladder contractility, occur in about $20 \%$ of patients. Prolonged QT intervals have been associated with bradycardia, although distinguishing disease-related from drug-related heart conduction effects may be difficult. Elevated fasting glucose and glycated hemoglobin levels are rarely encountered (Mazziotti et al., 2009). Pasireotide leads to reversible insulinopenia, hyperglycemia, and diabetes in $30 \%$ or more of patients (Gadelha et al., 2014; Silverstein, 2016).

2. Cushing Disease. Pituitary-dependent Cushing disease is caused by a corticotroph cell adenoma hypersecreting ACTH thus leading to adrenal cortisol overproduction (Biller et al., 2008). As corticotroph cells abundantly express $\mathrm{SST}_{5}$, pasireotide may suppress ACTH and features of hypercortisolemia in a subset of patients (Silverstein, 2016). In vitro, pasireotide inhibits basal and induced ACTH release from ACTHsecreting pituitary adenomas (Hofland et al., 2005; Batista et al., 2006). A double-blind, randomized phase III trial in 162 Cushing disease patients treated with pasireotide 600-900 $\mu \mathrm{g}$ twice daily showed that median urinary-free cortisol (UFC) levels were suppressed by $50 \%$, whereas $\sim 24 \%$ exhibited normalized UFC levels for 6 months. Patients with mildly elevated UFC levels are most likely to respond (Colao et al., 2012). Most patients not controlled within 8 weeks did not achieve control by study end. Of 75 patients with a demonstrable pituitary mass receiving $900 \mu \mathrm{g}$ pasireotide, $44 \%$ exhibited decreased mean pituitary tumor size. Blood pressure, weight, and quality of life improved, and triglyceride and low-density lipoprotein levels were reduced. Blood glucose and glycated hemoglobin levels increased in 118 of 162 patients, despite suppression of cortisol levels (Colao et al., 2012). As Cushing disease hypercortisolism is associated with insulin resistance and heart failure, monitoring of blood sugar and electrocardiograms for corrected QT interval prolongation and bradycardia is important.

3. Thyroid-Stimulating Hormone-Secreting Pituitary Adenomas. Central hyperthyroidism is caused by a TSHoma, a rare disease occurring both in children and adults. TSHomas typically have strong $\mathrm{SST}_{2}$ and often $\mathrm{SST}_{5}$ expression and show a good response to firstgeneration SRIF analogs, with about $10 \%$ of cases showing resistance (Beck-Peccoz et al., 2013). There is a single case in which cure was achieved (Fliers et al., 2012). Pasireotide has also been used successfully in TSHoma (van Eersel et al., 2017).

4. Neuroendocrine Tumors. Carcinoid, GI, and pancreatic NETs express cell surface $\mathrm{SST}_{2}$ (Öberg and Lamberts, 2016). These tumors exhibit significant morbidity and mortality, and at diagnosis fewer than $50 \%$ are surgically resectable (Kim et al., 2010). These tumors secrete 5-hydroxytryptamine or peptide hormones with significant clinical sequelae, including GI, bronchial, and cardiac dysfunction. Accordingly, SRIF analog therapy is aimed at decreasing or stabilizing tumor mass, as well as ameliorating adverse symptoms due to circulating hormones. Overall, survival of NET patients has improved about threefold since the introduction of SRIF analog therapy (Anthony et al., 1996; Yao et al., 2008).

In randomized double-blind trials, octreotide LAR and lanreotide autogel were shown to significantly ameliorate diarrhea or flushing in up to $80 \%$ of patients with carcinoid syndrome (Rubin et al., 1999; Modlin et al., 2006). In the placebo-controlled PROMID trial, when 85 patients with metastatic midgut NET received octreotide LAR, median time to tumor progression was extended from 6 to 14.3 months (Rinke et al., 2009). Furthermore, the disease was stabilized in two-thirds of patients receiving the SRIF analog therapy. In a 96-week trial by the Controlled Study of Lanreotide Antiproliferative Response in NET (CLARINET) of 204 patients randomized to receive placebo or lanreotide autogel $(120 \mathrm{mg}$ ), prolonged disease-free survival was demonstrated (Caplin et al., 2014). Interestingly, combination treatment of octreotide with everolimus, a mechanistic target of rapamycin kinase inhibitor, exhibited additive efficacy benefit, i.e., tumor volume reduction, in $75 \%$ of patients versus $45 \%$ for those receiving placebo plus octreotide (Pavel et al., 2011). Based on these results, SRIF analogs appear to offer both symptomatic improvement as well as direct antitumor effects in patients harboring NET.

\section{B. Factors Influencing Somatotropin-Release Inhibitory Factor Analog Resistance}

SRIF analog therapeutic efficacy rates vary depending on individual patient and tumor characteristics (Melmed, 2016). Understanding mechanisms driving SRIF analog responsiveness and resistance has enabled 
a personalized approach to acromegaly classification and management (Table 11) (Cuevas-Ramos et al., 2015). Retrospective studies have suggested predictors of acromegaly therapeutic responses, as well as markers of aggressive disease resistant to SRIF analogs that also correlate with adverse long-term outcomes. Increasing age, levels of GH and IGF-1, and tumor size are adverse determinants of SRIF analog responsiveness. As therapy is required to be open-ended, treatment duration is an important determinant of therapeutic sensitivity and control rates improve over years of treatment (Ayuk et al., 2002; Maiza et al., 2007).

In general, $\mathrm{SST}_{2}$ tumor expression correlates with SRIF analog responsiveness. Several studies have correlated efficacy in GH-secreting adenomas with $\mathrm{SST}_{2}$ immunostaining (Takei et al., 2007; Fougner et al., 2008b; Casarini et al., 2009; Casar-Borota et al., 2013; Gatto et al., 2013b). Choice of rabbit mAbs (Lupp et al., 2011; Chinezu et al., 2014; Iacovazzo et al., 2016) to assess patterns and distribution of membrane staining is also associated with SRIF analog responsiveness (Iacovazzo et al., 2016). In acromegaly patients resistant to octreotide, tumors lacking $\mathrm{SST}_{5}$ immunoreactivity were resistant to pasireotide, whereas those with $\mathrm{SST}_{5}$ staining using the rabbit $\mathrm{mAb} \mathrm{UMB}-4$ had superior biochemical response (Iacovazzo et al., 2016). Cell culture responses to octreotide and pasireotide correlated with $\mathrm{SST}_{2}$ and $\mathrm{SST}_{5}$ expression, and lower $\mathrm{SST}_{2}$ expression was associated with superior pasireotide effects (Gatto et al., 2017). However, in other in vitro studies on unselected pituitary tumor cell culture, responses to octreotide and pasireotide did not show an evident correspondence with the $\mathrm{SST}_{1}-\mathrm{SST}_{5}$ profile (Ibanez-Costa et al., 2016). Nevertheless, tumors immunopositive for $\mathrm{SST}_{2}$ expression are more likely to respond to octreotide and lanreotide (Brzana et al., 2013), and those with a higher $\mathrm{SST}_{2}$ to $\mathrm{SST}_{5}$ ratio show improved outcomes (Casar-Borota et al., 2013). SST mutation has only been described in a single patient with acromegaly, in which there was a missense (Arg240Trp) variant in the SSTR5 gene. The patient displayed resistance to

TABLE 11

Markers of somatostatin receptor ligand responsiveness in GH-secreting pituitary adenomas

Data adapted from Cuevas-Ramos et al. (2015).

\begin{tabular}{ll}
\hline GH Granulation & \multicolumn{1}{c}{$\begin{array}{c}\text { Dense vs. Sparse Using CAM5.2 } \\
\text { Cytokeratin Immunostaining }\end{array}$} \\
\hline $\mathrm{SST}_{2}, \mathrm{SST}_{5}$ & Positive vs. negative expression \\
$\mathrm{SST}_{2}: \mathrm{SST}_{5}$ & High vs. low ratio of average $\mathrm{SST}_{2}$ to $\mathrm{SST}_{5}$ \\
$\mathrm{SST}_{5} \mathrm{TMD}$ & Low vs. high expression \\
$\mathrm{AIP}$ & Lack vs. presence of mutation or high vs. low \\
& protein expression \\
$\beta$-arrestin & Low vs. high score based on intensity and \\
& expression pattern \\
Filamin A & High vs. low score based on intensity and \\
& expression pattern \\
Gsp & Presence vs. absence of mutation \\
E-cadherin & High vs. low score based on intensity and \\
& expression pattern \\
\hline
\end{tabular}

octreotide (Ballare et al., 2001). The cytoskeletal actinbinding scaffolding protein filamin A regulates $\mathrm{SST}_{2}$ trafficking and stability (Treppiedi et al., 2017). Lack of filamin A is associated with reduced cell surface expression of $\mathrm{SST}_{2}$ in neuroendocrine cell lines (Najib et al., 2012), although this was not the case in somatotroph cells that could be associated with SRIF analog resistance (Peverelli et al., 2014; Treppiedi et al., 2017).

Disrupted receptor recycling also alters SST signaling on the cell surface. Although GH-secreting adenoma $\mathrm{SST}_{2}$ expression may be less abundant following pretreatment with SRIF analogs (Casar-Borota et al., 2013), this does not appear to result in drug resistance in acromegaly and TSH-secreting adenomas, whereas tachyphylaxis has been observed in patients with NETs (Toumpanakis and Caplin, 2013).

Tumors with large, dense GH granules diffusely distributed throughout the cytosol are typically more responsive to SRIF analogs than are those containing small, uniform GH granules (Melmed et al., 1983). Sparsely GH granulated somatotrophinomas express less $\mathrm{SST}_{2}$ and more $\mathrm{SST}_{5}$ and are more resistant to SRIF analogs than those that are densely granulated (Fougner et al., 2012; Kato et al., 2012; Brzana et al., 2013; Larkin et al., 2013). They are larger and more invasive and occur in younger patients (Mayr et al., 2013). Low E-cadherin expression also correlates with sparsely granulated adenomas and SRIF analog resistance (Fougner et al., 2010). On magnetic resonance imaging, T2-weighted hyperintense GH-cell adenomas are frequently sparsely granulated and associated with a poor response to SRIF analogs (Hagiwara et al., 2003; Puig-Domingo et al., 2010; Heck et al., 2016a,b). Hypointense adenomas are smaller and less invasive than hyper- and isointense adenomas, but interestingly exhibit higher IGF-1 levels (Potorac et al., 2015).

Molecular markers, including low aryl hydrocarbon receptor-interacting protein (AIP) expression (JaffrainRea et al., 2013; Ritvonen et al., 2017), high $\beta$-arrestin expression (Gatto et al., 2013a), and presence of somatic mutation of the Gsp oncogene (Efstathiadou et al., 2015), have been associated with poor response to SRIF analogs, thus contributing to more adverse outcomes.

Octreotide stimulates Zac1 mRNA expression, whereas Zac1 knockdown renders cells unresponsive to SRIF analogs (Theodoropoulou et al., 2006). SRIF analogs upregulate AIP expression, and Aip mRNA correlates with Zac1 expression (Chahal et al., 2012), establishing a novel pathway (Gadelha et al., 2013). As reduced AIP expression may be associated with reduced $\mathrm{G}_{\mathrm{i} \alpha 2}$ levels (Tuominen et al., 2015; Ritvonen et al., 2017), SRIF analog resistance may be encountered in AIP mutation-positive patients (Leontiou et al., 2008; Daly et al., 2010; Oriola et al., 2012). Two truncated $\mathrm{SST}_{5}$ variants, $\mathrm{SST}_{5} \mathrm{TMD}_{4}$ with four TMD and sst 5 TMD5 with five TMD (DuránPrado et al., 2010), may inhibit $\mathrm{SST}_{2}$ functions. $\mathrm{SST}_{5} \mathrm{TMD} 4$ correlates inversely with $\mathrm{GH}$ and IGF-1 
reductions in response to octreotide LAR therapy (Durán-Prado et al., 2010; Luque et al., 2015).

Somatic guanine nucleotide-binding protein $\mathrm{G}_{\mathrm{s}}$ subunit $\alpha$ gene mutations, occurring in about $20 \%-$ $30 \%$ of somatotrophinomas, result in smaller, less invasive, and more densely granulated tumors, are more often seen in older patients, and respond more favorably to SRIF analogs (Landis et al., 1990; Barlier et al., 1998; Larkin et al., 2013). Guanine nucleotidebinding protein $\mathrm{G}_{\mathrm{s}}$ subunit $\alpha$ gene-positive patients have an approximately $10 \%$ greater reduction in $\mathrm{GH}$ levels in response to octreotide (Efstathiadou et al., 2015). In contrast, low levels of rapidly accelerated fibrosarcoma kinase inhibitory protein correlated with reduced octreotide responsiveness (Fougner et al., 2008a).

About 20\% of patients with Cushing disease achieve biochemical normalization with pasireotide (Colao et al., 2012). Corticotroph adenomas express high levels of $\mathrm{SST}_{5}$, followed by $\mathrm{SST}_{2}$ (Batista et al., 2006; de Bruin et al., 2009; Tateno et al., 2009; Lupp et al., 2011; van der Pas et al., 2013). As glucocorticoids may suppress $\mathrm{SST}_{2}$ expression (de Bruin et al., 2009), corticotrophinomas are usually resistant to octreotide or lanreotide. Although NETs usually express $\mathrm{SST}_{2}$, insulinomas have reduced expression of $\mathrm{SST}_{2}$ compared with other NETs (Hofland and Lamberts, 2003). $\mathrm{SST}_{2}$ is expressed in $90 \%$ of GI NETs, except insulinomas, where $50 \%$ of tumors express the receptor (Toumpanakis and Caplin, 2013). Tachyphylaxis has been described at variable time intervals after commencement of treatment, with reports ranging from 3 to 27 months (Toumpanakis and Caplin, 2013).

Assessment of clinical, imaging, biochemical, and histopathological markers therefore offers a personalized approach to predict biochemical outcomes with SRIF analogs (Puig Domingo, 2015; Melmed, 2016). Accordingly, rigorous phenotypic classification of acromegaly biomarkers for disease staging has been applied to further refine treatment approaches (Cuevas-Ramos et al., 2015; Giustina et al., 2016).

\section{Somatotropin-Release Inhibitory Factor- Based Radiopharmaceuticals}

1. Radiolabeled Agonists. Based on the metabolically stabilized synthetic octapeptide octreotide [D-PheCys-Phe-D-Nal-Lys-Thr-Cys-Thr(ol)], which displays high affinity for $\mathrm{SST}_{2}$ and moderate affinity for $\mathrm{SST}_{5}$ and $\mathrm{SST}_{3}$, Krenning et al. (1989) synthetized the ${ }^{123} \mathrm{I}-$ radioiodinated $\mathrm{Tyr}^{3}$ analog of octreotide $\left(\left[{ }^{123} \mathrm{I}^{\mathrm{T}} \mathrm{Tyr}^{3}\right.\right.$ SMS 201-995, $\left[{ }^{123} \mathrm{I}\right] \mathrm{Tyr}^{3}$-octreotide, $\left[{ }^{123} \mathrm{I}\right] \mathrm{TOC}$ ) (Fig. 24; Tables 12 and 13) and exploited this targeted radioligand for the first successful noninvasive single-photon emission computed tomography (SPECT) imaging of SST receptor-rich tumors in humans. This initial study on 10 patients is considered as pioneering work in the field of SST imaging, and also as general proof-of-concept for the usefulness of peptide receptor imaging (PRI) and as a starting point for development of radiolabeled ligands for targeted PRRT. With the aim to overcome the unsuitable biodistribution of this first tracer, which was caused by high lipophilicity, predominant hepatobiliary excretion, and thus undesirably high abdominal background activity, the same group successfully developed 2 years later in collaboration with a group at Sandoz Research Institute a new derivative, $\left[{ }^{111} \mathrm{In}\right]$ diethylenetriaminepentaaceticacidD-Phe ${ }^{1}$-octreotide ([ ${ }^{111}$ In]DTPA-D-Phe ${ }^{1}$-octreotide), named $\left.{ }^{[11} \mathrm{In}\right]$ pentetreotide (Bakker et al., 1991a,b) (Fig. 24). Subsequently, the favorable properties of this agent (e.g., ease of preparation, appropriate $t_{1 / 2}$, and absence of major accumulation in the upper abdominal region due to its renal clearance) were demonstrated in a comparative evaluation in humans (Krenning et al., 1992). For the first time, these studies introduced radiometals into the concept of PRI and PRRT, which significantly simplified the preparation of SST-targeted radiopharmaceuticals by

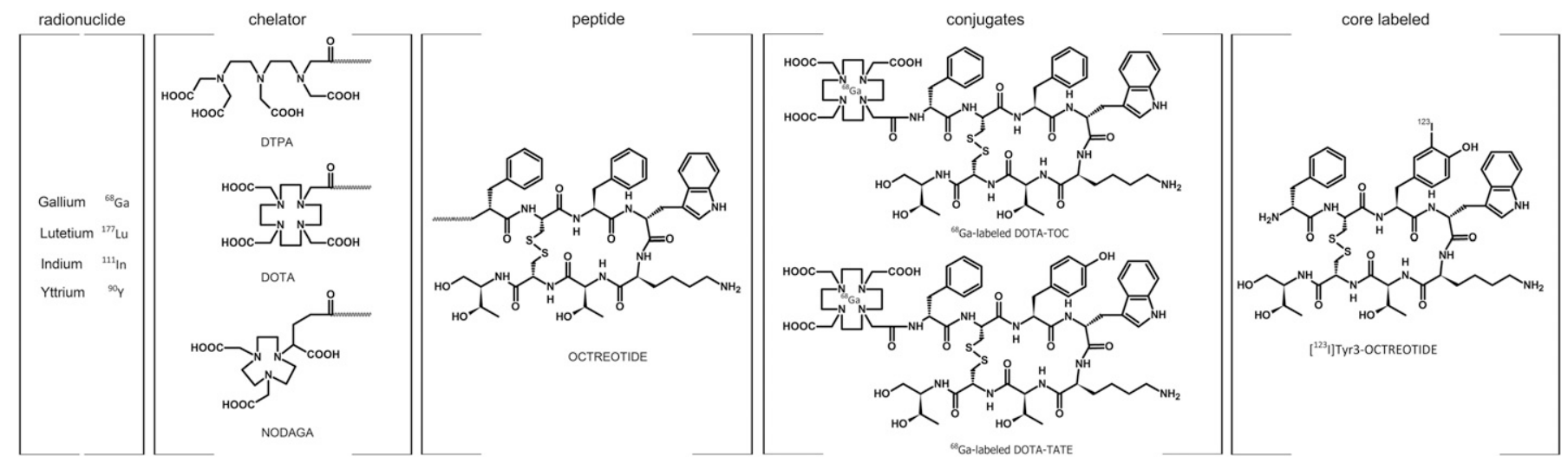

Fig. 24. Structures of SST ligands used for scintigraphy. $\left.{ }^{123} \mathrm{I}\right] \mathrm{T} y \mathrm{r} 3$-octroeotide, the very first compound for SST-targeted scintigraphy. Conjugation of DTPA to octreotide and labeling with indium-111 resulted in Octreoscan (Mallinckrodt), the first approved SST agent for SPECT imaging. Advanced Accelerator Application recently received market authorization for ${ }^{68} \mathrm{Ga}$-labeled DOTA-TOC (SomatoKit TOC) by the European Medicines Agency and for ${ }^{68} \mathrm{Ga}$-DOTA-TATE (Netspot) by the FDA. It is expected that $\left[{ }^{177} \mathrm{Lu}\right]$ DOTATATE will soon be approved by FDA and European Medicines Agency as first agent for peptide receptor radiotherapy. 
TABLE 12

Ligand-binding affinities of SRIF-based radiochemicals

\begin{tabular}{|c|c|c|c|c|c|c|}
\hline & $\mathrm{SST}_{1}$ & $\mathrm{SST}_{2}$ & $\mathrm{SST}_{3}$ & $\mathrm{SST}_{4}$ & $\mathrm{SST}_{5}$ & Regulatory Status \\
\hline \multicolumn{7}{|c|}{ Agonists with Predominant $\mathrm{SST}_{2}$ Affinity } \\
\hline DTPA-octreotide $^{a}$ & $>10,000$ & $12 \pm 2$ & $376 \pm 84$ & $>1000$ & $299 \pm 50$ & \\
\hline DOTA-lanreotide $^{a}$ & $>10,000$ & $26 \pm 3.4$ & $771 \pm 229$ & $>10,000$ & $73 \pm 12$ & \\
\hline In-DTPA-OC ${ }^{a}$ & $>10,000$ & $22 \pm 3.6$ & $182 \pm 13$ & $>1000$ & $237 \pm 52$ & FDA approved \\
\hline DOTA-TOC $^{a}$ & $>10,000$ & $14 \pm 2.6$ & $880 \pm 324$ & $>1000$ & $393 \pm 84$ & \\
\hline Y-DOTA-TOC ${ }^{a}$ & $>10,000$ & $11 \pm 1.7$ & $389 \pm 135$ & $>10,000$ & $114 \pm 29$ & Phase II studies \\
\hline DOTA-OC $^{a}$ & $>10,000$ & $14 \pm 3$ & $27 \pm 9$ & $>1000$ & $103 \pm 39$ & \\
\hline Y-DOTA-OC ${ }^{a}$ & $>10,000$ & $20 \pm 2$ & $27 \pm 8$ & $>10,000$ & $57 \pm 22$ & \\
\hline Ga-DOTA-TOC $^{a}$ & $>10,000$ & $2.5 \pm 0.5$ & $613 \pm 140$ & $>1000$ & $73 \pm 21$ & EMA approved \\
\hline Ga-DOTA-OC ${ }^{a}$ & $>10,000$ & $7.3 \pm 1.9$ & $120 \pm 45$ & $>1000$ & $60 \pm 14$ & \\
\hline DTPA-TATE $^{a}$ & $>10,000$ & $3.9 \pm 1$ & $>10,000$ & $>1000$ & $>1000$ & \\
\hline In-DTPA-TATE ${ }^{a}$ & $>10,000$ & $1.3 \pm 0.2$ & $>10,000$ & $433 \pm 16$ & $>1000$ & \\
\hline DOTA-TATE $^{a}$ & $>10,000$ & $1.5 \pm 0.4$ & $>1000$ & $453 \pm 176$ & $547 \pm 160$ & \\
\hline Y-DOTA-TATE ${ }^{a}$ & $>10,000$ & $1.6 \pm 0.4$ & $>1000$ & $523 \pm 239$ & $187 \pm 50$ & \\
\hline In-DOTA-TOC ${ }^{b}$ & $>10,000$ & $4.6 \pm 0.2$ & $120 \pm 26$ & $230 \pm 82$ & $130 \pm 17$ & \\
\hline Ga-DOTA-TATE $^{a}$ & $>10,000$ & $0.2 \pm 0.04$ & $>1000$ & $300 \pm 140$ & $377 \pm 18$ & FDA approved \\
\hline Lu-DOTATATE $^{c}$ & $>1000$ & $2.0 \pm 0.8$ & $162 \pm 16$ & $>1000$ & $>1000$ & Phase III completed \\
\hline I-Gluc-TOC ${ }^{d}$ & - & $2.2 \pm 0.7$ & $357 \pm 22$ & - & $64 \pm 24$ & \\
\hline I-Gluc-TTE $^{d}$ & - & $2.0 \pm 0.5$ & $>1000$ & - & $521 \pm 269$ & \\
\hline I-Gluc-S-TATE ${ }^{d}$ & - & $2.0 \pm 0.7$ & $398 \pm 19$ & - & $310 \pm 156$ & \\
\hline I-Gal-S-TATE ${ }^{d}$ & - & $2.0 \pm 0.8$ & $491 \pm 63$ & - & $413 \pm 167$ & \\
\hline Gluc-Lys(FP)-TATE ${ }^{e}$ & $>10,000$ & $2.8 \pm 0.4$ & $>1000$ & $437 \pm 84$ & $123 \pm 8.8$ & \\
\hline \multicolumn{7}{|c|}{ Agonists with Pansomatostatin-Like Binding Profile } \\
\hline Ga-DOTA-NOC $^{f}$ & $>10,000$ & $1.9 \pm 0.4$ & $40.0 \pm 5.8$ & $260 \pm 74$ & $7.2 \pm 1.6$ & Phase II studies \\
\hline In-DOTA-NOC ${ }^{g}$ & $>1000$ & $3.3 \pm 0.3$ & $26 \pm 1.9$ & $>1000$ & $10.4 \pm 1.6$ & \\
\hline In-DOTA-BOC ${ }^{g}$ & $>10,000$ & $3.1 \pm 0.3$ & $12 \pm 1.0$ & $455 \pm 65$ & $6 \pm 1.8$ & \\
\hline $\mathrm{NOC}^{-\mathrm{ATE}^{b}}$ & $>1000$ & $3.6 \pm 1.6$ & $302 \pm 137$ & $260 \pm 95$ & $16.7 \pm 9.9$ & \\
\hline${\mathrm{BOC}-\mathrm{ATE}^{b}}^{b}$ & $>1000$ & $0.8 \pm 0.4$ & $33 \pm 5.5$ & $80 \pm 20$ & $3.6 \pm 1.5$ & \\
\hline In-DOTA-NOC-ATE ${ }^{b}$ & $>10,000$ & $2 \pm 0.35$ & $13 \pm 4$ & $160 \pm 3.8$ & $4.3 \pm 0.5$ & \\
\hline Lu-DOTA-NOC-ATE ${ }^{d}$ & - & $3.6 \pm 0.3$ & $31 \pm 2$ & - & $15 \pm 1$ & \\
\hline In-DOTA-BOC-ATE ${ }^{b}$ & $>1000$ & $1.4 \pm 0.37$ & $5.5 \pm 0.8$ & $135 \pm 32$ & $3.9 \pm 0.2$ & \\
\hline Lu-DOTA-BOC-ATE ${ }^{d}$ & - & $2.4 \pm 0.3$ & $11 \pm 1$ & - & $8.3 \pm 0.4$ & \\
\hline KE $108^{h}$ & $0.96 \pm 0.15$ & $0.4 \pm 0.04$ & $0.44 \pm 0.06$ & $0.6 \pm 0.03$ & $0.26 \pm 0.04$ & \\
\hline $\mathrm{KE} 121^{h}$ & $1.6 \pm 0.7$ & $0.5 \pm 0.2$ & $0.3 \pm 0.1$ & $0.4 \pm 0.2$ & $0.2 \pm 0.1$ & \\
\hline Y-DOTA-K121 ${ }^{h}(\mathrm{Y}-\mathrm{KE} 88)$ & $2 \pm 0.8$ & $4.3 \pm 0.8$ & $0.7 \pm 0.2$ & $0.5 \pm 0.2$ & $0.7 \pm 0.2$ & \\
\hline Ga-DOTA-K121 ${ }^{h}$ (Ga-KE88) & $3.5 \pm 1.6$ & $1.8 \pm 1.6$ & $0.8 \pm 0.3$ & $1.8 \pm 0.5$ & $0.9 \pm 0.2$ & \\
\hline Y-DOTAGA-KE121 ${ }^{h}$ (Y-KE87) & $6.7 \pm 2.1$ & $2.7 \pm 2.4$ & $0.6 \pm 0.1$ & $1.6 \pm 0.6$ & $1.3 \pm 0.4$ & \\
\hline \multicolumn{7}{|c|}{ Antagonists } \\
\hline In-DOTA-BASS $^{i}$ & $>1000$ & $9.4 \pm 0.4$ & $>1000$ & $380 \pm 57$ & $>1000$ & Pilot study \\
\hline In-DOTA-JR $11^{j}$ & $>1000$ & $3.8 \pm 0.7$ & $>1000$ & $>1000$ & $>1000$ & Pilot study \\
\hline Ga-DOTA-JR11 $^{j}$ (Ga-OPS201) & $>1000$ & $29 \pm 2.7$ & $>1000$ & $>1000$ & $>1000$ & Pilot study \\
\hline Ga-NODAGA-JR11 ${ }^{j}$ (Ga-OPS202) & $>1000$ & $1.2 \pm 0.2$ & $>1000$ & $>1000$ & $>1000$ & Phase I/II study \\
\hline Lu-DOTA-JR11 ${ }^{j}$ (Lu-OPS201) & $>1000$ & $0.73 \pm 0.15$ & $>1000$ & $>1000$ & $>1000$ & \\
\hline $\mathrm{sst}_{3}-\mathrm{ODN}-8^{d}$ & - & $>1000$ & $6.7 \pm 2.6$ & & $>1000$ & \\
\hline DOTA-sst 3 -ODN-8 ${ }^{g}$ & $>1000$ & $>1000$ & $5.2 \pm 1.3$ & $>1000$ & $>1000$ & \\
\hline In-DOTA-sst ${ }_{3}-\mathrm{ODN}-8^{g}$ & $>1000$ & $>1000$ & $15 \pm 5.2$ & $>1000$ & $>1000$ & \\
\hline
\end{tabular}

EMA, European Medicines Agency.

${ }^{a}$ Data from Reubi et al. (2000a)

${ }^{b}$ Data from Ginj et al. (2005).

${ }^{c}$ Data from Schottelius et al. (2015).

${ }^{d}$ Data from Cescato et al. (2006).

${ }^{e}$ Data from Wester et al. (2003).

${ }^{f}$ Data from Antunes et al. (2007).

${ }^{g}$ Data from Ginj et al. (2006a).

${ }^{h}$ Data from Ginj et al. (2008).

${ }^{i}$ Data from Ginj et al. (2006b).

${ }^{j}$ Data from Fani et al. (2012).

exploiting fast and simple complexation procedures using chelator-conjugated peptide precursors, e.g., DTPA conjugated to the N-terminal D-Phe ${ }^{1}$-amino acid of the peptide as in $\left[{ }^{111} \mathrm{In}\right]$ pentetreotide. Data on $\left.{ }^{[111} \mathrm{In}\right]$ pentetreotide imaging in more than 1000 patients were published in 1993 (Krenning et al., 1993), and this is still the most frequently cited paper from the European Journal of Nuclear Medicine. Because the sensitivity and specificity of $\left[{ }^{111} \mathrm{In}\right]$ pentetreotide SPECT in patients with GEPNETs were higher than those obtained with the classic imaging modalities computer tomography or magnetic resonance imaging, $\left[{ }^{111} \mathrm{In}\right]$ pentetreotide (OctreoScan; Mallinckrodt, Staines-Upon-Thames, U.K.) was approved by the Food and Drug Administration (FDA) in 1994 as the first peptide-based imaging radiopharmaceutical on the basis of a dataset obtained in 350 European patients.

After having established a noninvasive imaging methodology for NETs, the next logical step was the development of a treatment option, similar to the imaging and treatment of thyroid cancer with ${ }^{123} \mathrm{I}$ - and ${ }^{131}$ I-iodide, respectively. Despite promising initial results after treatment of patients with very high doses of 
TABLE 13

Amino acid sequences of SRIF-based radiochemicals

Amino acids: first letter capitalized: L-amino acid; first letter in lowercase: D-amino acid.

\begin{tabular}{|c|c|c|c|c|c|c|c|c|c|c|c|}
\hline Ligand & Chelator/Prosthetic Group & AA1 & AA2 & AA3 & AA4 & AA5 & AA6 & AA7 & AA8 & AA9 & AA10 \\
\hline \multicolumn{12}{|c|}{ Peptide Agonists with Predominantly $\mathrm{SST}_{2}$ Affinity } \\
\hline Octreotide & - & & & phe & Cys & Phe & $\operatorname{trp}$ & Lys & Thr & Cys & Thr-ol \\
\hline Tyr3-octreotide (TOC) & & & & phe & Cys & Tyr & $\operatorname{trp}$ & Lys & Thr & Cys & Thr-ol \\
\hline RC160 (Vapreotide) & & & & phe & Cys & Tyr & $\operatorname{trp}$ & Lys & Val & Cys & $\operatorname{Trp}-\mathrm{NH}_{2}$ \\
\hline Lanreotide (BIM-23014) & & & & 2-nal & Cys & Tyr & $\operatorname{trp}$ & Lys & Val & Cys & Thr- $\mathrm{NH}_{2}$ \\
\hline \multicolumn{12}{|c|}{ Radiopeptide Agonists and Precursors with Predominantly $\mathrm{SST}_{2}$ Affinity } \\
\hline DTPA-octreotide & DTPA & & & phe & Cys & Phe & $\operatorname{trp}$ & Lys & Thr & Cys & Thr-ol \\
\hline DOTA-lanreotide & DOTA & & & 2-nal & Cys & Tyr & $\operatorname{trp}$ & Lys & Val & Cys & Thr-NH \\
\hline In-DTPA-OC & In-DTPA & & & phe & Cys & Phe & $\operatorname{trp}$ & Lys & Thr & Cys & Thr-ol \\
\hline DOTA-TOC & DOTA & & & phe & Cys & Tyr & $\operatorname{trp}$ & Lys & Thr & Cys & Thr-ol \\
\hline Y-DOTA-TOC & Y-DOTA & & & phe & Cys & Tyr & $\operatorname{trp}$ & Lys & Thr & Cys & Thr-ol \\
\hline DOTA-OC & DOTA & & & phe & Cys & Phe & $\operatorname{trp}$ & Lys & Thr & Cys & Thr-ol \\
\hline Y-DOTA-OC & Y-DOTA & & & phe & Cys & Phe & $\operatorname{trp}$ & Lys & Thr & Cys & Thr-ol \\
\hline Ga-DOTA-TOC & Ga-DOTA & & & phe & Cys & Tyr & $\operatorname{trp}$ & Lys & Thr & Cys & Thr-ol \\
\hline Ga-DOTA-OC & Ga-DOTA & & & phe & Cys & Phe & $\operatorname{trp}$ & Lys & Thr & Cys & Thr-ol \\
\hline DTPA-TATE & DTPA & & & phe & Cys & Tyr & $\operatorname{trp}$ & Lys & Thr & Cys & Thr \\
\hline In-DTPA-TATE & In-DTPA & & & phe & Cys & Tyr & $\operatorname{trp}$ & Lys & Thr & Cys & Thr \\
\hline DOTA-TATE & DOTA & & & phe & Cys & Tyr & $\operatorname{trp}$ & Lys & Thr & Cys & Thr \\
\hline Y-DOTA-TATE & Y-DOTA & & & phe & Cys & Tyr & $\operatorname{trp}$ & Lys & Thr & Cys & Thr \\
\hline In-DOTA-TOC & In-DOTA & & & phe & Cys & Tyr & $\operatorname{trp}$ & Lys & Thr & Cys & Thr-ol \\
\hline Ga-DOTA-TATE & Ga-DOTA & & & phe & Cys & Tyr & $\operatorname{trp}$ & Lys & Thr & Cys & Thr \\
\hline Lu-DOTATATE & Lu-DOTA & & & phe & Cys & Tyr & $\operatorname{trp}$ & Lys & Thr & Cys & Thr \\
\hline I-Gluc-TOC & Glucosyl & & & phe & Cys & 3-I-Tyr & $\operatorname{trp}$ & Lys & Thr & Cys & Thr-ol \\
\hline I-Gluc-TATE & Glucosyl & & & phe & Cys & 3-I-Tyr & $\operatorname{trp}$ & Lys & Thr & Cys & Thr \\
\hline I-Gluc-S-TATE & Glucosyl-S- & & & phe & Cys & 3-I-Tyr & $\operatorname{trp}$ & Lys & Thr & Cys & Thr \\
\hline I-Gal-S-TATE & Galactosyl-S- & & & phe & Cys & 3-I-Tyr & $\operatorname{trp}$ & Lys & Thr & Cys & Thr \\
\hline Gluc-Lys(FP)-TATE & $\begin{array}{c}\text { Glucosyl- } \\
\text { Lys(fluoropropionyl) }\end{array}$ & & & phe & Cys & 3-I-Tyr & $\operatorname{trp}$ & Lys & Thr & Cys & Thr \\
\hline
\end{tabular}

\begin{tabular}{|c|c|c|c|c|c|c|c|c|c|c|c|}
\hline \multicolumn{12}{|c|}{ Agonists toward Pansomatostatin-Like Binding Profile } \\
\hline Ga-DOTA-NOC & Ga-DOTA & & & phe & Cys & $1-\mathrm{Nal}$ & $\operatorname{trp}$ & Lys & Thr & Cys & Thr-ol \\
\hline In-DOTA-NOC & In-DOTA & & & phe & Cys & $1-\mathrm{Nal}$ & $\operatorname{trp}$ & Lys & Thr & Cys & Thr-ol \\
\hline In-DOTA-BOC & In-DOTA & & & phe & Cys & BzThi & $\operatorname{trp}$ & Lys & Thr & Cys & Thr-ol \\
\hline NOC-ATE & & & & phe & Cys & $1-\mathrm{Nal}$ & $\operatorname{trp}$ & Lys & Thr & Cys & Thr \\
\hline BOC-ATE & & & & phe & Cys & BzThi & $\operatorname{trp}$ & Lys & Thr & Cys & Thr-ol \\
\hline In-DOTA-NOC-ATE & In-DOTA & & & phe & Cys & $1-\mathrm{Nal}$ & $\operatorname{trp}$ & Lys & Thr & Cys & Thr \\
\hline Lu-DOTA-NOC-ATE & Lu-DOTA & & & phe & Cys & $1-\mathrm{Nal}$ & $\operatorname{trp}$ & Lys & Thr & Cys & Thr \\
\hline In-DOTA-BOC-ATE & In-DOTA & & & phe & Cys & BzThi & $\operatorname{trp}$ & Lys & Thr & Cys & Thr-ol \\
\hline Lu-DOTA-BOC-ATE & Lu-DOTA & & & phe & Cys & BzThi & $\operatorname{trp}$ & Lys & Thr & Cys & Thr-ol \\
\hline KE108 & Y-DOTA & Tyr & dab & Arg & Phe & Phe & $\operatorname{trp}$ & Lys & Thr & Phe & \\
\hline KE121 & & & dab & Arg & Phe & Phe & $\operatorname{trp}$ & Lys & Thr & Phe & \\
\hline Y-DOTA-K121 (Y-KE88) & Y-DOTA & & dab & Arg & Phe & Phe & $\operatorname{trp}$ & Lys & Thr & Phe & \\
\hline $\begin{array}{l}\text { Ga-DOTA-K121 (Ga- } \\
\text { KE88) }\end{array}$ & Y-DOTA & & dab & Arg & Phe & Phe & $\operatorname{trp}$ & Lys & Thr & Phe & \\
\hline $\begin{array}{l}\text { Y-DOTAGA-KE121 (Y- } \\
\text { KE87) }\end{array}$ & Y-DOTA & & $\mathrm{dab}$ & Arg & Phe & Phe & $\operatorname{trp}$ & Lys & Thr & Phe & \\
\hline \multicolumn{12}{|l|}{ Antagonists } \\
\hline In-DOTA-BASS & In-DOTA & & & $\mathrm{pNO}_{2}^{-} \mathrm{Phe}$ & cys & Tyr & $\operatorname{trp}$ & Lys & Thr & Cys & tyr- $\mathrm{NH}_{2}$ \\
\hline In-DOTA-JR11 & In-DOTA & & & $\mathrm{Cpa}$ & cys & Aph(Hor) & $\mathrm{Aph}(\mathrm{Cbm})$ & Lys & Thr & Cys & tyr- $\mathrm{NH}_{2}$ \\
\hline $\begin{array}{l}\text { Ga-DOTA-JR11 (Ga- } \\
\text { OPS201) }\end{array}$ & Ga-DOTA & & & Cpa & cys & Aph(Hor) & $\mathrm{Aph}(\mathrm{Cbm})$ & Lys & Thr & Cys & tyr- $\mathrm{NH}_{2}$ \\
\hline $\begin{array}{l}\text { Ga-NODAGA-JR11 (Ga- } \\
\text { OPS202) }\end{array}$ & Ga-NODAGA & & & Cpa & cys & $\operatorname{Aph}(\mathrm{Hor})$ & $\operatorname{Aph}(\mathrm{Cbm})$ & Lys & Thr & Cys & tyr- $\mathrm{NH}_{2}$ \\
\hline $\begin{array}{l}\text { Lu-DOTA-JR11 (Lu- } \\
\text { OPS201) }\end{array}$ & Lu-DOTA & & & Cpa & cys & Aph(Hor) & $\mathrm{Aph}(\mathrm{Cbm})$ & Lys & Thr & Cys & tyr- $\mathrm{NH}_{2}$ \\
\hline $\mathrm{sst}_{3}-\mathrm{ODN}-8$ & & $\mathrm{NH}_{2} \mathrm{CO}$ & cys & Phe & Tyr & $\begin{array}{l}\text { DAgl8(Me,2- } \\
\text { naphthoyl) }\end{array}$ & Lys & Thr & Phe & Cys & \\
\hline DOTA-sst $_{3}-\mathrm{ODN}-8$ & DOTA & $\mathrm{NH}_{2} \mathrm{CO}$ & cys & Phe & Tyr & $\begin{array}{l}\text { DAgl8(Me,2- } \\
\text { naphthoyl) }\end{array}$ & Lys & Thr & Phe & Cys & \\
\hline DOTA-TOC & DOTA & D-Phe & Cys & Phe & D-Trp & Lys & Thr & Cys & Thr(ol) & & \\
\hline
\end{tabular}

AoI $\alpha$-Aminoglycyl; Aph(Cbm), 4-aminocarbamoylphenylalanine; Aph(Hor), 4-amino-L-hydroorotylphenylalanine; BOC, [BzThi3]-octreotide; BOC-ATE, [BzThi3]-

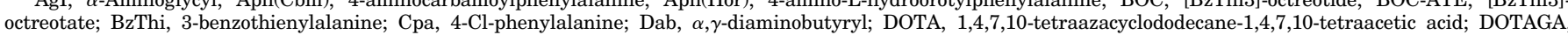

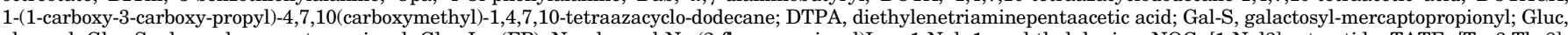

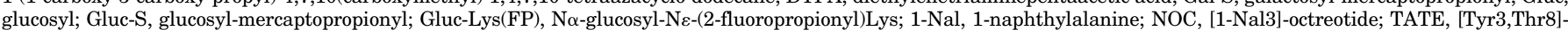
octreotide; TOC, [Tyr3]-octreotide.

$\left[{ }^{111} \mathrm{In}\right]$ pentetreotide (Valkema et al., 2002) (up to $2.7 \mathrm{Ci}$ in total) by means of the Auger and conversion electrons emitted by ${ }^{111} \mathrm{In}$, it became apparent that more efficient $\beta$-emitters, such as ${ }^{90} \mathrm{Y}$-yttrium $\left(t_{1 / 2}=64.1\right.$ hours,
$\mathrm{E} \beta_{\max }=2.28 \mathrm{MeV}$ ), might be better suited for SSTtargeted PRRT (Otte et al., 1997; Paganelli et al., 1999; Waldherr et al., 2001; Barone et al., 2005; Baum et al., 2012). These developments were based on the successful 
evaluation of 1,4,7,10-tetraazacyclododecane-1,4,7,10tetraacetic acid (DOTA) as chelator for therapeutic radiometals with improved thermodynamic and kinetic stability, suitable for all commonly used M(III) radiometals, such as ${ }^{90} \mathrm{Y}$ and ${ }^{177} \mathrm{Lu}$ for PRRT, ${ }^{111} \mathrm{In}$ for PRI with SPECT, and ${ }^{68} \mathrm{Ga}$ for PRI with positron emisson tomography (PET), to mention only a few (Albert et al., 1998). Shortly after the introduction of ${ }^{90}$ Y-labeled SST ligands for peptide receptor therapy, it became apparent that methods to reduce the renal tracer uptake and thus to protect the kidneys were needed. Hammond et al. (1993) were the first who introduced the concept of coinfusion of Lys/Arg solutions to reduce the renal uptake of $\left[{ }^{111} \mathrm{In}\right]$ Octreoscan by $>55 \%$. Despite this nephron protection, some patients suffered from renal failure after ${ }^{90} \mathrm{Y}$-DOTA-D-Tyr ${ }^{3}$-octreotide (DOTATOC) treatment. To overcome these limitations, ${ }^{90} \mathrm{Y}$ was substituted by ${ }^{177} \mathrm{Lu}\left[t_{1 / 2}=6.71\right.$ days, $\mathrm{E} \beta_{\max }=497 \mathrm{keV}$, $\mathrm{E} \gamma=113 \mathrm{keV}(6.4 \%)$, and $208 \mathrm{keV}(11 \%)]$, a $\beta^{-}$emitter with shorter penetration depth and coemission of low energy photons, thus allowing therapy monitoring by means of SPECT. Consequently, Lu-labeled SST ligands, e.g., $\left[{ }^{177} \mathrm{Lu}\right]$ DOTA-Tyr ${ }^{3}$-octreotate $\left(\left[{ }^{177} \mathrm{Lu}\right]\right.$ DOTATATE$)$, became the PRRT agent of choice (Fig. 25).

Concomitantly, and with the aim to increase the SST affinity, to modify the SST-binding profile, and to increase the tumor uptake of radiolabeled SSTbinding peptides after administration of doses typically in the range of $15-30 \mathrm{nmol}$ for imaging and 100 $300 \mathrm{nmol}$ for PRRT, various independent structural modifications of octreotide were introduced during the last 20 years, three of which should be mentioned in this work: 1) $\mathrm{Tyr}^{3}$, introduced into octreotide by Krenning et al. (1989) to be able to label the peptide with radioiodine, was found to significantly improve binding affinity and thus replaced D-Phe3 in a variety of subsequent octreotide analogs; 2) $\mathrm{Thr}^{8}(\mathrm{ol})$, originally introduced to increase the in vivo stability of octreotide, was substituted by $\mathrm{Thr}^{8}$, leading to $\mathrm{Tyr}^{3}$-octreotate (TATE); and 3) substitution of $\mathrm{Tyr}^{3}$ by 1-naphthylalanin, resulting in DOTA-D-Nal ${ }^{3}$-octreotide (DOTANOC) ligands (i.e., $\left[{ }^{68} \mathrm{Ga}\right] \mathrm{DOTANOC}$ ) with somewhat increased lipophilicity but high affinity to $\mathrm{SST}_{2}, \mathrm{SST}_{3}$, and $\mathrm{SST}_{5}$ (see below). Until today, the above-mentioned three DOTAconjugated peptides, DOTATOC, DOTATATE, and DOTANOC, are the most often clinically used SST ligands for imaging (e.g., ${ }^{68}$ Ga-labeled DOTATOC, DOTATATE, and DOTANOC) and therapy (e.g., ${ }^{177} \mathrm{Lu}-$ labeled DOTATOC and DOTATATE) (Antunes et al., 2007; Schottelius et al., 2015).

The first clinical studies with $\left[{ }^{177} \mathrm{Lu}\right]$ DOTATATE started in 2000 in Rotterdam and formed the basis of a multinational neuroendocrine tumors therapy phase III trial named NETTER-1 (Strosberg et al., 2017) at 41 global sites. NETTER-1 demonstrated that $\left[{ }^{177} \mathrm{Lu}\right]$ DOTATATE significantly improved progression-free survival (compared with octreotide injection, Sandostatin
LAR; $60 \mathrm{mg}$; Novartis, Basel, Switzerland) in patients with advanced midgut NETs.

Additional eight-amino-acid-containing peptide agonists such as lanreotide and vapreotide have been developed but never achieved relevance for PRI and PRRT (Breeman et al., 2001). In 2016, a "shake and bake" kit preparation of ${ }^{68} \mathrm{Ga}$-labeled $\left(t_{1 / 2}=68\right.$ minute) DOTATOC (Netspot; Advanced Accelerator Applications, Saint-Genis-Pouilly, France) was approved by the FDA for PET imaging of NETs, whereas the corresponding ${ }^{68} \mathrm{Ga}$-kit of the $\mathrm{Thr}^{8}$-analog $\left[{ }^{68} \mathrm{Ga}\right]$ DOTATATE was approved by the European Medicines Agency (SomaKIT TOC; Advanced Accelerator Applications). In addition, attempts were undertaken to optimize targeting properties of the first SST-imaging agent, $\left[{ }^{123}\right] \mathrm{Tyr}^{3}$ octreotide, and to transfer this tracer methodology to other radiohalogens, i.e., to the most commonly used PET radioisotope, fluorine-18 ( $t_{1 / 2}=109.7$ minutes $)$. Unfortunately, until recently, methods for direct radiofluorination were not available, and complex multistep preparations, which are inherently hard to automate, were developed. To overcome the limitations accompanied by radioiodination and conjugation of peptides with typically used ${ }^{18} \mathrm{~F}$-labeled prosthetic groups, i.e., a significant increase in lipophilicity and the resulting suboptimal imaging characteristics, Schottelius et al. (2004) adapted an interesting approach developed in the early 1990s by Albert et al. (1993) to improve oral availability of SST-binding peptides by glycosylation. Compensation of the negative influence of radiohalogenation by the use of carbohydrated peptides was highly effective, both for radioiodinated and ${ }^{18} \mathrm{~F}$-SST ligands (Wester et al., 2003, 2004; Schottelius et al., 2004), resulting in radiopharmaceuticals with excellent imaging properties. In a pilot study in 25 patients, $\mathrm{N} \alpha$-(1-deoxy-Dfructosyl)-N $\varepsilon-\left(2-\left[{ }^{18} \mathrm{~F}\right]\right.$ fluoropropionyl)-Lys ${ }^{0}-\mathrm{Tyr}^{3}$-octreotate PET (Gluc-Lys $\left[{ }^{18} \mathrm{~F}\right] \mathrm{FP}-\mathrm{TATE}$ ) allowed fast, high-contrast imaging of SST-positive tumors. The biokinetics and diagnostic performance of Gluc-Lys $\left(\left[{ }^{18} \mathrm{~F}\right] \mathrm{FP}\right)$-TATE was superior to [ [111 In]DTPA-octreotide and comparable with $\left[{ }^{68} \mathrm{Ga}\right]$ DOTATOC (Meisetschläger et al., 2006).

To improve imaging quality and the availability of a suitable SPECT imaging agent, ${ }^{99 \mathrm{~m}} \mathrm{Tc}$-labeled analogs of octreotide $\left(t_{1 / 2}=6\right.$ hours), such as ${ }^{99 \mathrm{~m}}$ Tc-tricineHYNIC-Tyr ${ }^{3}$-octreotide (Bangard et al., 2000), have been developed and successfully established for routine clinical use (Trejtnar et al., 2000; Gabriel et al., 2003; Hicks, 2010). Furthermore, [ ${ }^{99 m}$ Tc] ethylenediamine-N, $\mathrm{N}^{\prime}$-diacetic acid/HYNIC-Tyr ${ }^{3}$-octreotide (Tektrotyd, NCBJ RC POLATOM) has been approved in a number of European and non-European countries.

2. Pan Somatotropin-Release Inhibitory Factor-Like Peptides. Radiolabeled ligands that bind with similar high affinity to all five SSTs, so-called panSRIF-like ligands, are expected to expand the clinical indications of currently applied predominantly $\mathrm{SST}_{2}$-targeted ligands and to significantly improve tumor targeting, 

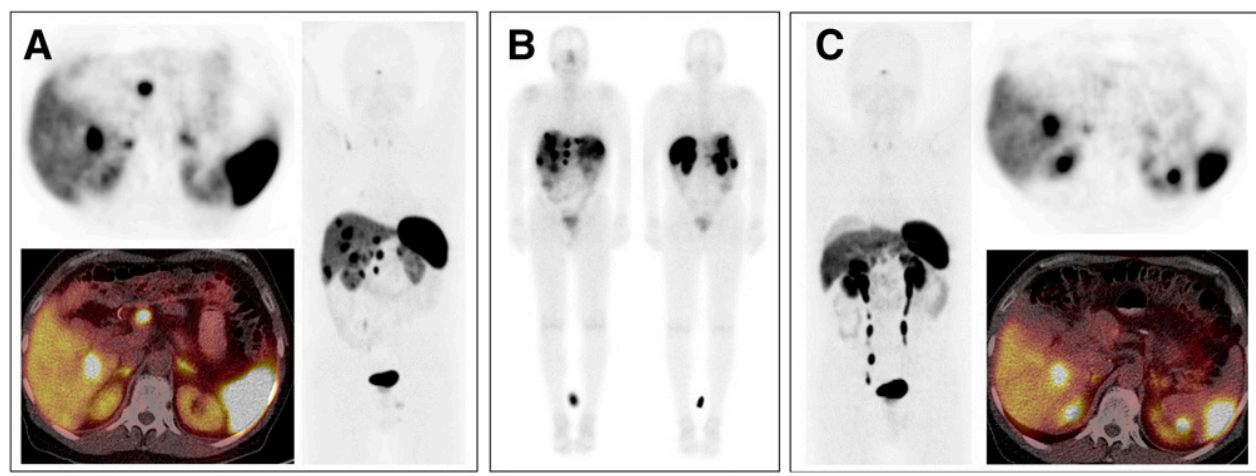

Fig. 25. Representative examples of clinical PRRT images. PRRT in a 73-year-old patient with metastasized neuroendocrine cancer (G1). Pretherapeutic 68Ga-DOTANOC PET/computed tomography images show extensive metastases in the liver and additional abdominal lymph node metastases (A). Post-therapeutic whole-body scintigraphy after application of 177Lu-DOTATATE confirms uptake in metastatic lesions (B). After four cycles of 177Lu-DOTATATE, 68Ga-DOTANOC PET/computed tomography demonstrates considerable response of liver and abdominal lymph node metastases (C). Images courtesy of M. Eiber, Department of Nuclear Medicine, Technical University Munich, Germany.

imaging sensitivity, and therapeutic efficacy by crossreactivity to coexpressed $\mathrm{SST}_{1}, \mathrm{SST}_{3}, \mathrm{SST}_{4}$, and $\mathrm{SST}_{5}$. One of the first developments in that direction was $\left.{ }^{[111} \mathrm{In}\right]$ DOTANOC (Wild et al., 2003), which showed high affinity to $\mathrm{SST}_{2}, \mathrm{SST}_{3}$, and $\mathrm{SST}_{5}$, and finally resulted in $\left[{ }^{68} \mathrm{Ga}\right] D O T A N O C$ (Wild et al., 2005), one of the most frequently used PET-imaging agents (already mentioned above), whereas the use of other compounds, such as DOTA-1-naphthylalanin, $\mathrm{Thr}^{8}$-octreotide (DOTANOC-ATE) or DOTA-BzThi ${ }^{3}, \mathrm{Thr}^{8}$-octreotide (DOTABOC-ATE) with even higher and broader affinity remained limited in use (Ginj et al., 2005, 2006a). Fani et al. (2010) developed bicyclic analogs, such as DOTA-Tyr-cyclo(DAB-Arg-cyclo(Cys-Phe-D-Trp-Lys-ThrCys)) (AM3; affinity for $\mathrm{SST}_{2}, \mathrm{SST}_{3}$, and $\mathrm{SST}_{5}$ ). The authors concluded that due to its rapid background clearance and high tumor to nontumor ratios, ${ }^{68} \mathrm{Ga}-\mathrm{AM} 3$ might be an ideal PET-imaging agent (Fani et al., 2010). The first such peptide with high-affinity binding for all five receptor subtypes was KE108 [Y-DOTA-Tyr-cyclo(D-DabArg-Phe-Phe-D-Trp-Lys-Thr-Phe)] (Reubi et al., 2002) and its DOTA analog [ $\left.{ }^{111} \mathrm{In}\right] \mathrm{KE} 88$ (Ginj et al., 2008). Unfortunately, this peptide was only efficiently internalized in $\mathrm{SST}_{3}$-expressing cells and did not offer pan-receptor-imaging properties. Another cyclic peptide, DOTA-pasireotide with affinity for four SST subtypes $\left(\mathrm{SST}_{1}, \mathrm{SST}_{2}, \mathrm{SST}_{3}\right.$, and $\left.\mathrm{SST}_{5}\right)$, has also been evaluated, with limited success. For both pasireotideand KE108-based radioligands, the absence of $\mathrm{SST}_{2}$ internalization may turn out to be a serious disadvantage and compromise their accumulation in target cells, because in most cases $\mathrm{SST}_{2}$ overexpression prevails.

Recently, native SRIF-14 and its D-Trp ${ }^{8}$ analog were considered for ligand development. Not unexpectedly, [111 In]DOTA-SS14 and [ $\left.{ }^{111} \mathrm{In}\right]$ DOTA-DTrp ${ }^{8}$-SS14 showed high affinity to all human SST subtypes, and $\left[{ }^{111} \mathrm{In}\right]$ DOTA-DTrp ${ }^{8}$-SS14 localized in experimental tumors, which selectively expressed rat $\mathrm{SST}_{2}$, human $\mathrm{SST}_{2}, \mathrm{SST}_{3}$, and $\mathrm{SST}_{5}$ (Tatsi et al., 2012). Furthermore, Maina et al. (2014) evaluated the SRIF mimic [ $\left.{ }^{111} \mathrm{In}\right]$ DOTA-LTT-SS28

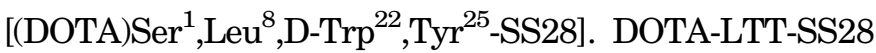
exhibited a panSRIF-like binding profile $\left(\mathrm{IC}_{50}\right.$ values for all SST subtypes in the low nanomolar range); behaved as an agonist at human $\mathrm{SST}_{2}, \mathrm{SST}_{3}$, and $\mathrm{SST}_{5}$; and efficiently stimulated internalization of the three receptor subtypes. In addition, significant and specific uptake was observed in HEK293-SST $2^{-}, \mathrm{HEK}^{293-\mathrm{SST}_{3}-\text {, and HEK293-SST }} 5^{-}$ expressing tumors. The authors concluded that $\left[{ }^{111} \mathrm{In}\right.$-DOTA]LTT-SS28 might be a promising ligand for multi-SST $1-\mathrm{SST}_{5}$-targeted tumor imaging.

Taking into account that high in vivo stability of a peptide radiopharmaceutical is of utmost importance for successful tumor imaging and PRRT, the re-evaluation of native or slightly modified SRIF-14 and SRIF-28 needs to be considered in the context of peptidase activity in vivo. Neutral endopeptidase is responsible for rapid breakdown of i.v. administered SRIF-, bombesin-, and gastrin-derived peptides, and activity of neutral endopeptidase can be overcome through the mere coinjection of a protease inhibitor, such as phosphoramidone (Nock et al., 2014). This approach may result in enhanced supply and accumulation of these radiopeptides at tumor sites and in increased clinical diagnostic sensitivity and therapeutic efficacy (Kaloudi et al., 2015, 2016).

3. Antagonists. Radiolabeled antagonists for imaging of cerebral receptor systems were established early in the application of noninvasive SPECT and PET imaging (Wagner et al., 1983). Internalization of the receptor after radioligand binding has been assumed to be critical for efficient retention of peptide radiopharmaceuticals in tumor cells and a prerequisite for efficient PRI and PRRT imaging. Almost all SST-binding peptide lead structures exploited for radiopharmaceutical development originate from development of SST-targeting drugs (e.g., octreotide) and exhibited agonistic behavior. The first results indicating that high-affinity SST antagonists that poorly internalize into tumor cells perform more effectively than corresponding agonists that are highly internalized into tumor cells were considered to be at the 
forefront of a paradigm shift in nuclear oncology imaging (Ginj et al., 2006b).

Motivated by a study of Bass et al. (1996), who found that inversion of the chirality at positions 1 and 2 of the octreotide peptide family converts an agonist to an antagonist, and by structure activity relationship studies of Hocart et al. (1998, 1999), the first radiolabeled SST antagonists were evaluated (Cescato et al., 2008). Ginj et al. (2006b) showed that two peptides with high affinity to $\mathrm{SST}_{2}\left(\left[{ }^{111} \mathrm{In}\right] \mathrm{DOTA}-\mathrm{sst}_{2}\right.$-ANT) and $\mathrm{SST}_{3}$ ([ $\left.\left.{ }^{111} \mathrm{In}\right] \mathrm{DOTA}-\mathrm{sst}_{3}-\mathrm{ODN}-8\right)$, respectively, did not trigger $\mathrm{SST}_{2}$ - or $\mathrm{SST}_{3}$-mediated internalization and prevented agonist-stimulated internalization. Subsequent biodistribution studies in mice bearing $\mathrm{SST}_{3}$-expressing tumors revealed strong accumulation of [ $\left.{ }^{111} \mathrm{In}\right]$ DOTA$\mathrm{SST}_{3}-\mathrm{ODN}-8$ at 1 hour with up to $60 \%$ of injected radioactivity per gram of tissue and maintained at a high level for $>72$ hours, whereas $\left[{ }^{111} \mathrm{In}\right]$ DOTA-NOC, with strong $\mathrm{SST}_{3}$-binding and internalization properties, showed a much lower and shorter-lasting uptake in $\mathrm{SST}_{3}$-expressing tumors. The same tendency was seen for $\mathrm{SST}_{2}$-binding ligand $\left[{ }^{111} \mathrm{In}\right] \mathrm{DOTA}-\mathrm{SST}_{2}$-ANT when compared with the highly potent $\mathrm{SST}_{2}$-selective agonist $\left[{ }^{111} \mathrm{In}\right]$ DTPA-TATE, suggesting that this observation may be valid for more than just one particular GPCR.

A pilot study in five patients with NETs or thyroid cancer provided the first evidence that SST imaging with [ $\left.{ }^{111} \mathrm{In}\right]$ DOTA-sst ${ }_{2}$-ANT ([ $\left.{ }^{111} \mathrm{In}\right]$ DOTA-BASS) is significantly more sensitive and effective than that employing the FDA-approved radiotracer ${ }^{111}$ In-DTPAoctreotide (OctreoScan; Mallinckrodt) (Wild et al., 2011). In a comprehensive preclinical study, three different $\mathrm{SST}_{2}$ antagonists, LM3 ( $p$-Cl-Phe-cyclo(D-Cys-Tyr-D$\mathrm{Aph}(\mathrm{Cbm})-\mathrm{Lys}-\mathrm{Th}$-Cys)D-Tyr-NH ${ }_{2}$, JR10 ( $p$-NO $\mathrm{N}_{2}$-Phec[D-Cys-Tyr-D-Aph(Cbm)-Lys-Thr-Cys]-D-Tyr-NH ${ }_{2}$, and JR11 (Cpa-c[D-Cys-Aph(Hor)-D-Aph(Cbm)-Lys-Thr-Cys]D-Tyr- $\mathrm{NH}_{2}$ ), were evaluated in combination with two chelators [DOTA and 1,4,7-triazacyclononane,1-glutaric acid-4,7-acetic acid (NODAGA)] and various (radio)metals [In(III), Y(III), Lu(III), Cu(II), and Ga(III)]. Although the antagonists were found to be very sensitive to chelator modifications and complexation with distinct radiometals (Fani et al., 2012), the study illustrated the potential of the antagonists, because even a low-affinity antagonist was shown to be slightly superior to a high-affinity agonist, outweighing the affinity differences. This is due to the fact that a neutral antagonist labels receptors in all states (active or inactive), whereas an agonist only labels receptors in an active conformation. The active conformation may represent a limited proportion of the whole population, because most GPCRs show low levels of constitutive activity.

JR11 was selected for clinical development as a PETimaging agent labeled with ${ }^{68} \mathrm{Ga}$ using the chelator NODAGA $\left({ }^{68} \mathrm{Ga}-\mathrm{NODAGA-JR} 11\right.$ or $\left.{ }^{68} \mathrm{Ga}-\mathrm{OPS} 202\right)$ and as a therapeutic agent labeled with ${ }^{177} \mathrm{Lu}$ using the chelator DOTA ( ${ }^{177} \mathrm{Lu}-\mathrm{DOTA}-J R 11$ or $\left.{ }^{177} \mathrm{Lu}-\mathrm{OPS} 201\right)$.
In a preclinical comparison of the antagonist $\left[{ }^{177} \mathrm{Lu}\right] \mathrm{OPS} 201$ (DOTA-JR11; DOTA-[Cpa-c(DCys-Aph(Hor)$\left.\mathrm{DAph}(\mathrm{Cbm})-\mathrm{Lys}-\mathrm{Thr}-\mathrm{Cys})-\mathrm{DTyr}-\mathrm{NH}_{2}\right]$ ) and the $\mathrm{SST}_{2}$ agonist $\left[{ }^{177} \mathrm{Lu}\right]$ DOTATATE, the antagonist showed 2.5-times higher tumor dose, longer tumor residence time, and 1.3-fold higher tumor-to-kidney dose ratio (Nicolas et al., 2017). A phase I/II PET/computed tomography study for interindividual comparison of ${ }^{68} \mathrm{Ga}$-NODAGA-JR11 $\left({ }^{68} \mathrm{Ga}\right.$-OPS202) and ${ }^{68} \mathrm{Ga}-D O T A T O C$ indicated increased image contrast, sensitivity, and diagnostic accuracy of ${ }^{68} \mathrm{Ga}$-OPS202 for staging of gastroenteropancreatic NETs (Nicolas et al., 2018). The theranostic pair ${ }^{68} \mathrm{Ga}$-DOTA-JR11 and ${ }^{177} \mathrm{Lu}$-DOTA-JR11 has also been investigated in NET patients, and ${ }^{177}$ Lu-DOTA-JR11 $\left({ }^{177} \mathrm{Lu}\right.$-OPS201) is being evaluated in phase I/II.

More than 20 years after approval of $\left[{ }^{111} \mathrm{In}\right]$ Octreoscan, recent regulatory approvals of $\left[{ }^{68} \mathrm{Ga}\right]$ DOTATATE and $\left[{ }^{68} \mathrm{Ga}\right]$ DOTATOC and the expected authorization of $\left[{ }^{177} \mathrm{Lu}\right]$ DOTATATE will significantly advance the field and stimulate further peptide receptor-based imaging and therapy options. With respect to tracer development, recent studies with radiolabeled antagonists have generated high expectations that require verification in detailed clinical studies. Combination of $\left[{ }^{177} \mathrm{Lu}\right]$ DOTATATE radiotherapy with chemotherapy, targeted agents, or immunotherapies has been initiated. Data of a first phase III study comparing the combination of ${ }^{177} \mathrm{Lu}$ PRRT and capecitabine (Xeloda; Roche, Basel, Switzerland), an oral chemotherapy agent, with ${ }^{177} \mathrm{Lu}$ PRRT alone started at Erasmus MC (Rotterdam, Netherlands) are expected in 2017 (van Essen et al., 2008). Further studies on combination therapies, named peptide receptor chemoradionuclide therapy, have recently been published (Kong et al., 2017).

\section{Conclusions}

The SRIF system comprises seven genes encoding two peptide precursors, SRIF and CST, and five receptors. Compared with many other regulatory peptides, this is a relatively high number of receptors. It remains an intriguing question why this system needs five different receptors to transduce the SRIF signal. To date, far few disease-associated mutations have been identified. KO mice for any of the SSTs exhibit rather mild phenotypes. This suggests a high functional redundancy with potential that loss of one SST can be compensated by another SST subtype. Although SSTs often show overlapping distributions, they exhibit striking differences in their subcellular localizations and trafficking. $\mathrm{SST}_{2}$ and $\mathrm{SST}_{5}$ receptors are primary targets for pharmacological treatment of pituitary adenomas and NETs. In addition, $\mathrm{SST}_{2}$ is a prototypical GPCR for development of peptide-based radiopharmaceuticals for diagnostic and therapeutic intervention. Consequently, the localization, regulation, and function of the five SSTs have 
been studied extensively in vitro and in vivo. However, open questions remain:

- Are there additional receptors for SRIF and/or CST?

- Does heterodimerization among SSTs and other GPCRs occur in vivo?

- Can SSTs signal from within intracellular compartments?

- What is the exact mechanism involved in the SST and AIP-Zac1 pathway?

- Why do sparsely granulated GH-secreting adenomas not respond well to SRIF analogs?

- Why do cell lines not respond well to SRIF analogs?

- What is the molecular mechanism underlying tumor imaging using SST antagonists?

- What is the therapeutic potential for development of biased SST agonists?

- Is there a potential for SST ligands that can penetrate the blood brain barrier and enter the CNS?

- How are targeting and membrane trafficking of $\mathrm{SST}_{1}$ regulated?

- What is the precise physiologic role of truncated $\mathrm{SST}_{5}$ variants?

The future challenges include deciphering crystal structures for the five SSTs to facilitate discovery of novel SST subtype-selective agonists and antagonists, which are both safe and effective. It is expected that novel delivery ligand systems including oral formulations and longer-acting injectables will offer enhanced patient convenience for long-term therapies. In the future, SRIF-based therapies may become available for novel indications, such as treatment of type 2 diabetes with $\mathrm{SST}_{5}$ antagonists or treatment of neuropathic pain with $\mathrm{SST}_{4}$ agonists.

\section{Acknowledgments}

We thank Joana Modrow and Désirée Koch for excellent support in literature search and for assistance in figure and text editing, and Christian H. Langnickel for preparing art work. We also thank Manuel D. Gahete for critical advice on Cortistatin section.

\section{Authorship Contributions}

Wrote or contributed to the writing of the manuscript: Günther, Tulipano, Dournaud, Bousquet, Csaba, Kreienkamp, Lupp, Korbonits, Castaño, Wester, Culler, Melmed, Schulz.

\section{References}

Afargan M, Janson ET, Gelerman G, Rosenfeld R, Ziv O, Karpov O, Wolf A, Bracha M, Shohat D, Liapakis G, et al. (2001) Novel long-acting somatostatin analog with endocrine selectivity: potent suppression of growth hormone but not of insulin. Endocrinology 142:477-486.

Albers AR, O'Dorisio MS, Balster DA, Caprara M, Gosh P, Chen F, Hoeger C, Rivier $J$, Wenger GD, O'Dorisio TM, et al. (2000) Somatostatin receptor gene expression in neuroblastoma. Regul Pept 88:61-73.

Albert R, Marbach P, Bauer W, Briner U, Fricker G, Bruns C, and Pless J (1993) SDZ CO 611: a highly potent glycated analog of somatostatin with improved oral activity. Life Sci 53:517-525.

Albert R, Smith-Jones P, Stolz B, Simeon C, Knecht H, Bruns C, and Pless J (1998) Direct synthesis of [DOTA-DPhe1]-octreotide and [DOTA-DPhe1,Tyr3]-octreotide
(SMT487): two conjugates for systemic delivery of radiotherapeutical nuclides to somatostatin receptor positive tumors in man. Bioorg Med Chem Lett 8:1207-1210. Alderton F, Humphrey PPA, and Sellers LA (2001) High-intensity p38 kinase activity is critical for $\mathrm{p} 21$ (cip1) induction and the antiproliferative function of G(i) proteincoupled receptors. Mol Pharmacol 59:1119-1128.

Alexander SP, Christopoulos A, Davenport AP, Kelly E, Marrion NV, Peters JA Faccenda E, Harding SD, Pawson AJ, Sharman JL, et al.; CGTP Collaborators (2017) The Cconcise Guide to Pharmacology 2017/18: G protein-coupled receptors. Br J Pharmacol 174 (Suppl 1):S17-S129.

Allen JP, Hathway GJ, Clarke NJ, Jowett MI, Topps S, Kendrick KM, Humphrey PP Wilkinson LS, and Emson PC (2003) Somatostatin receptor 2 knockout/lacZ knockin mice show impaired motor coordination and reveal sites of somatostatin action within the striatum. Eur $J$ Neurosci 17:1881-1895

Allia E, Tarabra E, Volante M, Cerrato M, Ghigo E, Muccioli G, and Papotti M (2005) Expression of cortistatin and MrgX2, a specific cortistatin receptor, in human neuroendocrine tissues and related tumours. J Pathol 207:336-345.

Ammon C, Schäfer J, Kreuzer OJ, and Meyerhof W (2002) Presence of a plasma membrane targeting sequence in the amino-terminal region of the rat somatostatin receptor 3. Arch Physiol Biochem 110:137-145.

Annamalai AK, Webb A, Kandasamy N, Elkhawad M, Moir S, Khan F, Maki-Petaja K, Gayton EL, Strey CH, O'Toole S, et al. (2013) A comprehensive study of clinical, biochemical, radiological, vascular, cardiac, and sleep parameters in an unselected cohort of patients with acromegaly undergoing presurgical somatostatin receptor ligand therapy. J Clin Endocrinol Metab 98:1040-1050.

Anthony LB, Martin W, Delbeke D, and Sandler M (1996) Somatostatin receptor imaging: predictive and prognostic considerations. Digestion 57 (Suppl 1):50-53.

Antunes P, Ginj M, Zhang H, Waser B, Baum RP, Reubi JC, and Maecke H (2007) Are radiogallium-labelled DOTA-conjugated somatostatin analogues superior to those labelled with other radiometals? Eur J Nucl Med Mol Imaging 34:982-993.

Aoki T, Motoi F, Sakata N, Naitoh T, Katayose Y, Egawa S, Miyazaki J, and Unno M (2014) Somatostatin analog inhibits the growth of insulinoma cells by p27mediated G1 cell cycle arrest. Pancreas 43:720-729.

Aourz N, De Bundel D, Stragier B, Clinckers R, Portelli J, Michotte Y, and Smolders I (2011) Rat hippocampal somatostatin sst3 and sst4 receptors mediate anticonvulsive effects in vivo: indications of functional interactions with sst2 receptors. Neuropharmacology 61:1327-1333.

Aourz N, Portelli J, Coppens J, De Bundel D, Di Giovanni G, Van Eeckhaut A Michotte Y, and Smolders I (2014) Cortistatin-14 mediates its anticonvulsant effects via sst2 and sst3 but not ghrelin receptors. CNS Neurosci Ther 20:662-670.

Ardjomand N, Ardjomand N, Schaffler G, Radner H, and El-Shabrawi Y (2003) Expression of somatostatin receptors in uveal melanomas. Invest Ophthalmol Vis Sci 44:980-987.

Arena S, Pattarozzi A, Corsaro A, Schettini G, and Florio T (2005) Somatostatin receptor subtype-dependent regulation of nitric oxide release: involvement of different intracellular pathways. Mol Endocrinol 19:255-267.

Arena S, Pattarozzi A, Massa A, Esteve JP, Iuliano R, Fusco A, Susini C, and Florio T (2007) An intracellular multi-effector complex mediates somatostatin receptor 1 activation of phospho-tyrosine phosphatase eta. Mol Endocrinol 21:229-246.

Armani C, Catalani E, Balbarini A, Bagnoli P, and Cervia D (2007) Expression, pharmacology, and functional role of somatostatin receptor subtypes 1 and 2 in human macrophages. J Leukoc Biol 81:845-855.

Arne G, Nilsson B, Dalmo J, Kristiansson E, Arvidsson Y, Forssell-Aronsson E, Nilsson O, and Ahlman H (2013) Gastrointestinal stromal tumors (GISTs) express somatostatin receptors and bind radiolabeled somatostatin analogs. Acta Oncol 52 : $783-792$.

Atkinson H, England JA, Rafferty A, Jesudason V, Bedford K, Karsai L, and Atkin SL (2013) Somatostatin receptor expression in thyroid disease. Int J Exp Pathol 94: $226-229$.

Ayuk J, Stewart SE, Stewart PM, and Sheppard MC (2002) Long-term safety and efficacy of depot long-acting somatostatin analogs for the treatment of acromegaly. $J$ Clin Endocrinol Metab 87:4142-4146.

Azar R, Najib S, Lahlou H, Susini C, and Pyronnet S (2008) Phosphatidylinosito 3-kinase-dependent transcriptional silencing of the translational repressor $4 \mathrm{E}$ BP1. Cell Mol Life Sci 65:3110-3117.

Bakker WH, Albert R, Bruns C, Breeman WAP, Hofland LJ, Marbach P, Pless J, Pralet D, Stolz B, Koper JW, et al. (1991a) [111In-DTPA-D-Phe1]-octreotide, a potential radiopharmaceutical for imaging of somatostatin receptor-positive tumors: synthesis, radiolabeling and in vitro validation. Life Sci 49:1583-1591.

Bakker WH, Krenning EP, Reubi JC, Breeman WAP, Setyono-Han B, de Jong M, Kooij PPM, Bruns C, van Hagen PM, Marbach P, et al. (1991b) In vivo application of [111In-DTPA-D-Phe1]-octreotide for detection of somatostatin receptor-positive tumors in rats. Life Sci 49:1593-1601.

Ballarè E, Persani L, Lania AG, Filopanti M, Giammona E, Corbetta S, Mantovani S, Arosio M, Beck-Peccoz P, Faglia G, et al. (2001) Mutation of somatostatin receptor type 5 in an acromegalic patient resistant to somatostatin analog treatment. J Clin Endocrinol Metab 86:3809-3814.

Bangard M, Béhé M, Guhlke S, Otte R, Bender H, Maecke HR, and Biersack HJ (2000) Detection of somatostatin receptor-positive tumours using the new $99 \mathrm{mTc}-$ tricine-HYNIC-D-Phe1-Tyr3-octreotide: first results in patients and comparison with 111In-DTPA-D-Phe1-octreotide. Eur J Nucl Med 27:628-637.

Banno Y, Sasaki S, Kamata M, Kunitomo J, Miyamoto Y, Abe H, Taya N, Oi S, Watanabe M, Urushibara T, et al. (2017) Design and synthesis of a novel series of orally active, selective somatostatin receptor 2 agonists for the treatment of type 2 diabetes. Bioorg Med Chem 25:5995-6006.

Bär KJ, Schurigt U, Scholze A, Segond Von Banchet G, Stopfel N, Bräuer R, Halbhuber KJ, and Schaible HG (2004) The expression and localization of somatostatin receptors in dorsal root ganglion neurons of normal and monoarthritic rats. $\mathrm{Neu}$ roscience 127:197-206.

Baraban SC and Tallent MK (2004) Interneuron diversity series: interneuronal neuropeptides-endogenous regulators of neuronal excitability. Trends Neurosci 27: 135-142. 
Baratta MV, Lamp T, and Tallent MK (2002) Somatostatin depresses long-term potentiation and $\mathrm{Ca} 2+$ signaling in mouse dentate gyrus. $J$ Neurophysiol $\mathbf{8 8}$ : 3078-3086.

Barbieri F, Bajetto A, Pattarozzi A, Gatti M, Würth R, Thellung S, Corsaro A, Villa V, Nizzari M, and Florio T (2013) Peptide receptor targeting in cancer: the somatostatin paradigm. Int $J$ Pept 2013:926295.

Barbieri F, Pattarozzi A, Gatti M, Aiello C, Quintero A, Lunardi G, Bajetto A, Ferrari A, Culler MD, and Florio T (2009) Differential efficacy of SSTR1, -2, and -5 agonists in the inhibition of $\mathrm{C} 6$ glioma growth in nude mice. Am J Physiol Endocrinol Metab 297:E1078-E1088.

Barbieri F, Pattarozzi A, Gatti M, Porcile C, Bajetto A, Ferrari A, Culler MD, and Florio T (2008) Somatostatin receptors 1, 2, and 5 cooperate in the somatostatin inhibition of $\mathrm{C} 6$ glioma cell proliferation in vitro via a phosphotyrosine phosphatase-eta-dependent inhibition of extracellularly regulated kinase-1/2. En docrinology 149:4736-4746.

Barlier A, Gunz G, Zamora AJ, Morange-Ramos I, Figarella-Branger D, Dufour H, Enjalbert A, and Jaquet P (1998) Pronostic and therapeutic consequences of Gs alpha mutations in somatotroph adenomas. J Clin Endocrinol Metab 83:1604-1610.

Barnes K, Walkden BJ, Wilkinson TC, and Turner AJ (1997) Expression of endothelin-converting enzyme in both neuroblastoma and glial cell lines and its localization in rat hippocampus. $J$ Neurochem 68:570-577.

Barone R, Borson-Chazot F, Valkema R, Walrand S, Chauvin F, Gogou L, Kvols LK, Krenning EP, Jamar F, and Pauwels S (2005) Patient-specific dosimetry in predicting renal toxicity with (90)Y-DOTATOC: relevance of kidney volume and dose rate in finding a dose-effect relationship. J Nucl Med 46 (Suppl 1):99S-106S.

Basivireddy J, Somvanshi RK, Romero IA, Weksler BB, Couraud P-O, Oger J, and Kumar U (2013) Somatostatin preserved blood brain barrier against cytokine induced alterations: possible role in multiple sclerosis. Biochem Pharmacol 86:497-507.

Bass RT, Buckwalter BL, Patel BP, Pausch MH, Price LA, Strnad J, and Hadcock JR (1996) Identification and characterization of novel somatostatin antagonists. Mol Pharmacol 50:709-715.

Bassant MH, Simon A, Poindessous-Jazat F, Csaba Z, Epelbaum J, and Dournaud P (2005) Medial septal GABAergic neurons express the somatostatin sst2A receptor: functional consequences on unit firing and hippocampal theta. $J$ Neurosci 25 : 2032-2041.

Batista DL, Zhang X, Gejman R, Ansell PJ, Zhou Y, Johnson SA, Swearingen B, Hedley-Whyte ET, Stratakis CA, and Klibanski A (2006) The effects of SOM230 on cell proliferation and adrenocorticotropin secretion in human corticotroph pituitary adenomas. J Clin Endocrinol Metab 91:4482-4488.

Bauch C, Koliwer J, Buck F, Hönck HH, and Kreienkamp HJ (2014) Subcellular sorting of the G-protein coupled mouse somatostatin receptor 5 by a network of PDZ-domain containing proteins. PLoS One 9:e88529.

Bauer W, Briner U, Doepfner W, Haller R, Huguenin R, Marbach P, Petcher TJ, and Pless (1982) SMS 201-995: a very potent and selective octapeptide analogue of somatostatin with prolonged action. Life Sci 31:1133-1140.

Baum RP, Kulkarni HR, and Carreras C (2012) Peptides and receptors in imageguided therapy: theranostics for neuroendocrine neoplasms. Semin Nucl Med $\mathbf{4 2}$ 190-207.

Baumeister H, Kreuzer OJ, Roosterman D, Schäfer J, and Meyerhof W (1998) Cloning, expression, pharmacology and tissue distribution of the mouse somatostatin receptor subtype 5. J Neuroendocrinol 10:283-290.

Baumeister $\mathrm{H}$ and Meyerhof W (1998) Involvement of a Pit-1 binding site in the regulation of the rat somatostatin receptor 1 gene expression. Ann N Y Acad Sci 865:390-392

Baumeister H and Meyerhof W (2000a) Gene regulation of somatostatin receptors in rats. J Physiol Paris 94:167-177.

Baumeister H and Meyerhof W (2000b) The POU domain transcription factor Tst-1 activates somatostatin receptor 1 gene expression in pancreatic beta-cells. J Biol Chem 275:28882-28887.

Beaudet A, Greenspun D, Raelson J, and Tannenbaum GS (1995) Patterns of expression of SSTR1 and SSTR2 somatostatin receptor subtypes in the hypothalamus of the adult rat: relationship to neuroendocrine function. Neuroscience $\mathbf{6 5}$ 551-561

Beck-Peccoz P, Lania A, Beckers A, Chatterjee K, and Wemeau JL (2013) 2013 European thyroid association guidelines for the diagnosis and treatment of thyrotropin-secreting pituitary tumors. Eur Thyroid $J$ 2:76-82.

Ben-Shlomo A and Melmed S (2010) Pituitary somatostatin receptor signaling. Trends Endocrinol Metab 21:123-133.

Ben-Shlomo A, Pichurin O, Barshop NJ, Wawrowsky KA, Taylor J, Culler MD, Chesnokova V, Liu NA, and Melmed S (2007) Selective regulation of somatostatin receptor subtype signaling: evidence for constitutive receptor activation. Mol Endocrinol 21:2565-2578.

Ben-Shlomo A, Schmid H, Wawrowsky K, Pichurin O, Hubina E, Chesnokova V, Liu N-A, Culler M, and Melmed S (2009a) Differential ligand-mediated pituitary somatostatin receptor subtype signaling: implications for corticotroph tumor therapy. $J$ Clin Endocrinol Metab 94:4342-4350.

Ben-Shlomo A, Wawrowsky KA, Proekt I, Wolkenfeld NM, Ren S-G, Taylor J, Culler MD, and Melmed S (2005) Somatostatin receptor type 5 modulates somatostatin receptor type 2 regulation of adrenocorticotropin secretion. $J$ Biol Chem 280: 24011-24021.

Ben-Shlomo A, Zhou C, Pichurin O, Chesnokova V, Liu NA, Culler MD, and Melmed S (2009b) Constitutive somatostatin receptor activity determines tonic pituitary cell response. Mol Endocrinol 23:337-348.

Benali N, Cordelier P, Calise D, Pages P, Rochaix P, Nagy A, Esteve JP, Pour PM, Schally AV, Vaysse N, et al. (2000) Inhibition of growth and metastatic progression of pancreatic carcinoma in hamster after somatostatin receptor subtype 2 (sst2) gene expression and administration of cytotoxic somatostatin analog AN-238. Proc Natl Acad Sci USA 97:9180-9185.

Berbari NF, Johnson AD, Lewis JS, Askwith CC, and Mykytyn K (2008a) Identification of ciliary localization sequences within the third intracellular loop of $\mathrm{G}$ protein-coupled receptors. Mol Biol Cell 19:1540-1547.
Berbari NF, Lewis JS, Bishop GA, Askwith CC, and Mykytyn K (2008b) Bardet-Biedl syndrome proteins are required for the localization of $\mathrm{G}$ protein-coupled receptors to primary cilia. Proc Natl Acad Sci USA 105:4242-4246.

Bertherat J, Tenenbaum F, Perlemoine K, Videau C, Alberini JL, Richard B, Dousset B, Bertagna X, and Epelbaum J (2003) Somatostatin receptors 2 and 5 are the major somatostatin receptors in insulinomas: an in vivo and in vitro study. $J$ Clin Endocrinol Metab 88:5353-5360.

Bevan JS (2005) Clinical review: the antitumoral effects of somatostatin analog therapy in acromegaly. J Clin Endocrinol Metab 90:1856-1863.

Bhattarai JP, Kaszás A, Park SA, Yin H, Park SJ, Herbison AE, Han SK, and Abrahám IM (2010) Somatostatin inhibition of gonadotropin-releasing hormone neurons in female and male mice. Endocrinology 151:3258-3266.

Bigiani A, Petrucci C, Ghiaroni V, Dal Monte M, Cozzi A, Kreienkamp HJ, Richter D, and Bagnoli P (2004) Functional correlates of somatostatin receptor 2 overexpression in the retina of mice with genetic deletion of somatostatin receptor 1 . Brain Res 1025:177-185.

Biller BMK, Grossman AB, Stewart PM, Melmed S, Bertagna X, Bertherat J, Buchfelder M, Colao A, Hermus AR, Hofland LJ, et al. (2008) Treatment of adrenocorticotropin-dependent Cushing's syndrome: a consensus statement. J Clin Endocrinol Metab 93:2454-2462.

Binaschi A, Bregola G, and Simonato M (2003) On the role of somatostatin in seizure control: clues from the hippocampus. Rev Neurosci 14:285-301.

Bito H, Mori M, Sakanaka C, Takano T, Honda Z, Gotoh Y, Nishida E, and Shimizu T (1994) Functional coupling of SSTR4, a major hippocampal somatostatin receptor, to adenylate cyclase inhibition, arachidonate release and activation of the mitogenactivated protein kinase cascade. J Biol Chem 269:12722-12730.

Bläker M, Schmitz M, Gocht A, Burghardt S, Schulz M, Bröring DC, Pace A, Greten H, and De Weerth A (2004) Differential expression of somatostatin receptor subtypes in hepatocellular carcinomas. J Hepatol 41:112-118.

Bocci G, Culler MD, Fioravanti A, Orlandi P, Fasciani A, Colucci R, Taylor JE, Sadat D, Danesi R, and Del Tacca M (2007) In vitro antiangiogenic activity of selective somatostatin subtype-1 receptor agonists. Eur J Clin Invest 37:700-708.

Borbély E, Scheich B, and Helyes Z (2013) Neuropeptides in learning and memory. Neuropeptides 47:439-450.

Bourgin P, Fabre V, Huitrón-Reséndiz S, Henriksen SJ, Prospero-Garcia O, Criado JR, and de Lecea L (2007) Cortistatin promotes and negatively correlates with slow-wave sleep. Eur J Neurosci 26:729-738.

Bousquet C, Delesque N, Lopez F, Saint-Laurent N, Estève J-P, Bedecs K, Buscail L, Vaysse N, and Susini C (1998) sst2 somatostatin receptor mediates negative regulation of insulin receptor signaling through the tyrosine phosphatase SHP-1. $J$ Biol Chem 273:7099-7106.

Bousquet C, Guillermet-Guibert J, Saint-Laurent N, Archer-Lahlou E, Lopez F, Fanjul M, Ferrand A, Fourmy D, Pichereaux C, Monsarrat B, et al. (2006) Direct binding of p85 to sst2 somatostatin receptor reveals a novel mechanism for inhibiting PI3K pathway. EMBO J 25:3943-3954.

Braun H, Schulz S, Becker A, Schröder H, and Höllt V (1998) Protective effects of cortistatin (CST-14) against kainate-induced neurotoxicity in rat brain. Brain Res 803:54-60.

Braun M (2014) The somatostatin receptor in human pancreatic $\beta$-cells. Vitam Horm 95:165-193.

Brazeau P, Vale W, Burgus R, Ling N, Butcher M, Rivier J, and Guillemin R (1973) Hypothalamic polypeptide that inhibits the secretion of immunoreactive pituitary growth hormone. Science 179:77-79.

Breeman WA, de Jong M, Kwekkeboom DJ, Valkema R, Bakker WH, Kooij PP, Visser TJ, and Krenning EP (2001) Somatostatin receptor-mediated imaging and therapy: basic science, current knowledge, limitations and future perspectives. Eur J Nucl Med 28:1421-1429.

Briard N, Dutour A, Epelbaum J, Sauze N, Slama A, and Oliver C (1997) Species differences between male rat and ram pituitary somatostatin receptors involved in the inhibition of growth hormone secretion. Eur J Endocrinol 137:545-555.

Broglio F, Papotti M, Muccioli G, and Ghigo E (2007) Brain-gut communication: cortistatin, somatostatin and ghrelin. Trends Endocrinol Metab 18:246-251.

Brown M, Rivier J, and Vale W (1977) Somatostatin: analogs with selected biological activities. Science 196:1467-1469.

Bruno JF, Xu Y, Song J, and Berelowitz M (1992) Molecular cloning and functional expression of a brain-specific somatostatin receptor. Proc Natl Acad Sci USA 89 $11151-11155$

Bruno JF, Xu Y, Song J, and Berelowitz M (1993) Tissue distribution of somatostatin receptor subtype messenger ribonucleic acid in the rat. Endocrinology 133: 2561-2567.

Bruns C, Lewis I, Briner U, Meno-Tetang G, and Weckbecker G (2002) SOM230: a novel somatostatin peptidomimetic with broad somatotropin release inhibiting factor (SRIF) receptor binding and a unique antisecretory profile. Eur J Endocrinol 146:707-716.

Brzana J, Yedinak CG, Gultekin SH, Delashaw JB, and Fleseriu M (2013) Growth hormone granulation pattern and somatostatin receptor subtype $2 \mathrm{~A}$ correlate with postoperative somatostatin receptor ligand response in acromegaly: a large single center experience. Pituitary 16:490-498.

Buchan AM, Lin CY, Choi J, and Barber DL (2002) Somatostatin, acting at receptor subtype 1 , inhibits Rho activity, the assembly of actin stress fibers, and cell migration. J Biol Chem 277:28431-28438.

Burke PG, Li Q, Costin ML, McMullan S, Pilowsky PM, and Goodchild AK (2008) Somatostatin 2A receptor-expressing presympathetic neurons in the rostral ventrolateral medulla maintain blood pressure. Hypertension 52:1127-1133.

Buscail L, Bournet B, Vernejoul F, Cambois G, Lulka H, Hanoun N, Dufresne M, Meulle A, Vignolle-Vidoni A, Ligat L, et al. (2015) First-in-man phase 1 clinical trial of gene therapy for advanced pancreatic cancer: safety, biodistribution, and preliminary clinical findings. Mol Ther 23:779-789.

Buscail L, Delesque N, Estève JP, Saint-Laurent N, Prats H, Clerc P, Robberecht P, Bell GI, Liebow C, Schally AV, et al. (1994) Stimulation of tyrosine phosphatase and inhibition of cell proliferation by somatostatin analogues: mediation by human 
somatostatin receptor subtypes SSTR1 and SSTR2. Proc Natl Acad Sci USA 91: 2315-2319.

Buscail L, Saint-Laurent N, Chastre E, Vaillant JC, Gespach C, Capella G, Kalthoff H, Lluis F, Vaysse N, and Susini C (1996) Loss of sst2 somatostatin receptor gene expression in human pancreatic and colorectal cancer. Cancer Res 56:1823-1827.

Busco G, Cardone RA, Greco MR, Bellizzi A, Colella M, Antelmi E, Mancini MT, Dell'Aquila ME, Casavola V, Paradiso A, et al. (2010) NHE1 promotes invadopodial ECM proteolysis through acidification of the peri-invadopodial space. FASEB $J \mathbf{2 4}$ 3903-3915

Cai C, Li H, Kangasniemi A, Pihlajamaa T, Von Ossowski L, Kerkelä K, Schulz S, Rivera C, and Keinänen K (2008) Somatostatin receptor subtype 1 is a PDZ ligand for synapse-associated protein 97 and a potential regulator of growth cone dynamics. Neuroscience 157:833-843.

Calebiro D, Nikolaev VO, Gagliani MC, de Filippis T, Dees C, Tacchetti C, Persani L, and Lohse MJ (2009) Persistent cAMP-signals triggered by internalized G-proteincoupled receptors. PLoS Biol 7:e1000172.

Callaghan B and Furness JB (2014) Novel and conventional receptors for ghrelin, desacyl-ghrelin, and pharmacologically related compounds. Pharmacol Rev 66: 984-1001.

Cammalleri M, Martini D, Timperio AM, and Bagnoli P (2009) Functional effects of somatostatin receptor 1 activation on synaptic transmission in the mouse hippocampus. J Neurochem 111:1466-1477.

Caplin ME, Pavel M, Ćwikła JB, Phan AT, Raderer M, Sedláčková E, Cadiot G, Wolin EM, Capdevila J, Wall L, et al.; CLARINET Investigators (2014) Lanreotide in metastatic enteropancreatic neuroendocrine tumors. N Engl J Med 371:224-233.

Carlsen SM, Lund-Johansen M, Schreiner T, Aanderud S, Johannesen O, Svartberg J, Cooper JG, Hald JK, Fougner SL, and Bollerslev J; Preoperative Octreotide Treatment of Acromegaly Study Group (2008) Preoperative octreotide treatment in newly diagnosed acromegalic patients with macroadenomas increases cure shortterm postoperative rates: a prospective, randomized trial. J Clin Endocrinol Metab 93:2984-2990.

Carmichael JD, Bonert VS, Mirocha JM, and Melmed S (2009) The utility of oral glucose tolerance testing for diagnosis and assessment of treatment outcomes in 166 patients with acromegaly. J Clin Endocrinol Metab 94:523-527.

Carmichael JD, Bonert VS, Nuño M, Ly D, and Melmed S (2014) Acromegaly clinical trial methodology impact on reported biochemical efficacy rates of somatostatin receptor ligand treatments: a meta-analysis. J Clin Endocrinol Metab 99 1825-1833.

Caron PJ, Bevan JS, Petersenn S, Flanagan D, Tabarin A, Prévost G, Maisonobe P, and Clermont A; PRIMARYS Investigators (2014) Tumor shrinkage with lanreotide Autogel $120 \mathrm{mg}$ as primary therapy in acromegaly: results of a prospective multicenter clinical trial. J Clin Endocrinol Metab 99:1282-1290.

Carruthers AM, Warner AJ, Michel AD, Feniuk W, and Humphrey PP (1999) Activation of adenylate cyclase by human recombinant sst5 receptors expressed in CHO-K1 cells and involvement of Galphas proteins. $\mathrm{Br} J$ Pharmacol 126: 1221-1229.

Casar-Borota O, Heck A, Schulz S, Nesland JM, Ramm-Pettersen J, Lekva T, Alafuzoff I, and Bollerslev J (2013) Expression of SSTR2a, but not of SSTRs 1, 3, or 5 in somatotroph adenomas assessed by monoclonal antibodies was reduced by octreotide and correlated with the acute and long-term effects of octreotide. J Clin Endocrinol Metab 98:E1730-E1739.

Casarini AP, Jallad RS, Pinto EM, Soares IC, Nonogaki S, Giannella-Neto D, Musolino NR, Alves VA, and Bronstein MD (2009) Acromegaly: correlation between expression of somatostatin receptor subtypes and response to octreotide-lar treatment. Pituitary 12:297-303

Casini G, Catalani E, Dal Monte M, and Bagnoli P (2005) Functional aspects of the somatostatinergic system in the retina and the potential therapeutic role of somatostatin in retinal disease. Histol Histopathol 20:615-632.

Casini G, Dal Monte M, Petrucci C, Gambellini G, Grouselle D, Allen JP, Kreienkamp HJ, Richter D, Epelbaum J, and Bagnoli P (2004) Altered morphology of rod bipola cell axonal terminals in the retinas of mice carrying genetic deletion of somatostatin subtype receptor 1 or 2 . Eur $J$ Neurosci 19:43-54.

Castinetti F, Saveanu A, Morange I, and Brue T (2009) Lanreotide for the treatment of acromegaly. Adv Ther 26:600-612.

Cervera P, Videau C, Viollet C, Petrucci C, Lacombe J, Winsky-Sommerer R, Csaba Z, Helboe L, Daumas-Duport C, Reubi JC, et al. (2002) Comparison of somatostatin receptor expression in human gliomas and medulloblastomas. J Neuroendocrinol 14:458-471.

Cervia D and Bagnoli P (2007) An update on somatostatin receptor signaling in native systems and new insights on their pathophysiology. Pharmacol Ther 116:322-341.

Cervia D, Casini G, and Bagnoli P (2008) Physiology and pathology of somatostatin in the mammalian retina: a current view. Mol Cell Endocrinol 286:112-122.

Cervia D, Fiorini S, Pavan B, Biondi C, and Bagnoli P (2002) Somatostatin (SRIF) modulates distinct signaling pathways in rat pituitary tumor cells; negative coupling of SRIF receptor subtypes 1 and 2 to arachidonic acid release. Naunyn Schmiedebergs Arch Pharmacol 365:200-209.

Cescato R, Erchegyi J, Waser B, Piccand V, Maecke HR, Rivier JE, and Reubi JC (2008) Design and in vitro characterization of highly sst2-selective somatostatin antagonists suitable for radiotargeting. $J$ Med Chem 51:4030-4037.

Cescato R, Schulz S, Waser B, Eltschinger V, Rivier JE, Wester H-J, Culler M, Ginj M, Liu Q, Schonbrunn A, et al. (2006) Internalization of sst2, sst3, and sst5 receptors: effects of somatostatin agonists and antagonists. J Nucl Med 47:502-511.

Chahal HS, Trivellin G, Leontiou CA, Alband N, Fowkes RC, Tahir A, Igreja SC, Chapple JP, Jordan S, Lupp A, et al. (2012) Somatostatin analogs modulate AIP in somatotroph adenomas: the role of the ZAC1 pathway. J Clin Endocrinol Metab 97: E1411-E1420

Chalabi-Dchar M, Cassant-Sourdy S, Duluc C, Fanjul M, Lulka H, Samain R, Roche C, Breibach F, Delisle MB, Poupot M, et al. (2015) Loss of somatostatin receptor subtype 2 promotes growth of KRAS-induced pancreatic tumors in mice by activating PI3K signaling and overexpression of CXCL16. Gastroenterology 148: $1452-1465$
Charland S, Boucher MJ, Houde M, and Rivard N (2001) Somatostatin inhibits Akt phosphorylation and cell cycle entry, but not p42/p44 mitogen-activated protein (MAP) kinase activation in normal and tumoral pancreatic acinar cells. Endocrinology 142:121-128.

Chen L, Fitzpatrick VD, Vandlen RL, and Tashjian AH Jr (1997) Both overlapping and distinct signaling pathways for somatostatin receptor subtypes SSTR1 and SSTR2 in pituitary cells. $J$ Biol Chem 272:18666-18672.

Chen L and Tashjian AH Jr (1999) Identification of distinct signalling pathways for somatostatin receptors SSTR1 and SSTR2 as revealed by microphysiometry. Cell Signal 11:499-505.

Chinezu L, Vasiljevic A, Jouanneau E, Francois P, Borda A, Trouillas J, and Raverot G (2014) Expression of somatostatin receptors, SSTR2A and SSTR5, in 108 endocrine pituitary tumors using immunohistochemical detection with new specific monoclonal antibodies. Hum Pathol 45:71-77.

Christenn M, Kindler S, Schulz S, Buck F, Richter D, and Kreienkamp H-J (2007) Interaction of brain somatostatin receptors with the PDZ domains of PSD-95. FEBS Lett 581:5173-5177.

Ciganoka D, Balcere I, Kapa I, Peculis R, Valtere A, Nikitina-Zake L, Lase I, Schiöth HB, Pirags V, and Klovins J (2011) Identification of somatostatin receptor type 5 gene polymorphisms associated with acromegaly. Eur $J$ Endocrinol 165:517-525. Colao A, Auriemma RS, and Pivonello R (2016) The effects of somatostatin analogue therapy on pituitary tumor volume in patients with acromegaly. Pituitary 19: 210-221.

Colao A, Bronstein MD, Freda P, Gu F, Shen CC, Gadelha M, Fleseriu M, van der Lely AJ, Farrall AJ, Hermosillo Reséndiz K, et al.; Pasireotide C2305 Study Group (2014) Pasireotide versus octreotide in acromegaly: a head-to-head superiority study. J Clin Endocrinol Metab 99:791-799.

Colao A, Ferone D, Marzullo P, and Lombardi G (2004) Systemic complications of acromegaly: epidemiology, pathogenesis, and management. Endocr Rev 25:102-152.

Colao A, Petersenn S, Newell-Price J, Findling JW, Gu F, Maldonado M, Schoenherr U, Mills D, Salgado LR, and Biller BM; Pasireotide B2305 Study Group (2012) A 12 -month phase 3 study of pasireotide in Cushing's disease. $N$ Engl J Med 366: 914-924

Cole SL and Schindler M (2000) Characterisation of somatostatin sst2 receptor splice variants. J Physiol Paris 94:217-237.

Conlon JM, Tostivint H, and Vaudry H (1997) Somatostatin- and urotensin II-related peptides: molecular diversity and evolutionary perspectives. Regul Pept 69:95-103. Cordelier P, Estève JP, Bousquet C, Delesque N, O'Carroll AM, Schally AV, Vaysse N, Susini C, and Buscail L (1997) Characterization of the antiproliferative signal mediated by the somatostatin receptor subtype sst5. Proc Natl Acad Sci USA 94: 9343-9348.

Cordelier P, Estève JP, Najib S, Moroder L, Vaysse N, Pradayrol L, Susini C, and Buscail L (2006) Regulation of neuronal nitric-oxide synthase activity by somatostatin analogs following $\mathrm{SST}_{5}$ somatostatin receptor activation. J Biol Chem 281:19156-19171.

Córdoba-Chacón J, Gahete MD, Castaño JP, Kineman RD, and Luque RM (2012a) Homologous and heterologous in vitro regulation of pituitary receptors for somatostatin, growth hormone $(\mathrm{GH})$-releasing hormone, and ghrelin in a nonhuman primate (Papio anubis). Endocrinology 153:264-272.

Córdoba-Chacón J, Gahete MD, Culler MD, Castaño JP, Kineman RD, and Luque RM (2012b) Somatostatin dramatically stimulates growth hormone release from primate somatotrophs acting at low doses via somatostatin receptor 5 and cyclic AMP. J Neuroendocrinol 24:453-463.

Córdoba-Chacón J, Gahete MD, Durán-Prado M, Luque RM, and Castaño JP (2011) Truncated somatostatin receptors as new players in somatostatin-cortistatin pathophysiology. Ann N Y Acad Sci 1220:6-15.

Córdoba-Chacón J, Gahete MD, Durán-Prado M, Pozo-Salas AI, Malagón MM, Gracia-Navarro F, Kineman RD, Luque RM, and Castaño JP (2010) Identification and characterization of new functional truncated variants of somatostatin receptor subtype 5 in rodents. Cell Mol Life Sci 67:1147-1163.

Corleto VD, Falconi M, Panzuto F, Milione M, De Luca O, Perri P, Cannizzaro R, Bordi C, Pederzoli P, Scarpa A, et al. (2009) Somatostatin receptor subtypes 2 and 5 are associated with better survival in well-differentiated endocrine carcinomas. Neuroendocrinology 89:223-230.

Coy DH, Meyers C, Arimura A, Schally AV, and Redding TW (1978) Observations on the growth hormone, insulin, and glucagon release-inhibiting activities of somatostatin analogues. Metabolism 27 (9 Suppl 1):1407-1410.

Criado JR, Li H, Jiang X, Spina M, Huitrón-Reséndiz S, Liapakis G, Calbet M, Siehler S, Henriksen SJ, Koob G, et al. (1999) Structural and compositional determinants of cortistatin activity. $J$ Neurosci Res 56:611-619.

Crider AM and Witt KA (2007) Somatostatin sst4 ligands: chemistry and pharmacology. Mini Rev Med Chem 7:213-220.

Cronin MJ, Rogol AD, Myers GA, and Hewlett EL (1983) Pertussis toxin blocks the somatostatin-induced inhibition of growth hormone release and adenosine $3^{\prime}, 5^{\prime}$ monophosphate accumulation. Endocrinology 113:209-215.

Csaba Z, Bernard V, Helboe L, Bluet-Pajot MT, Bloch B, Epelbaum J, and Dournaud $\mathrm{P}$ (2001) In vivo internalization of the somatostatin sst2A receptor in rat brain: evidence for translocation of cell-surface receptors into the endosomal recycling pathway. Mol Cell Neurosci 17:646-661.

Csaba Z and Dournaud P (2001) Cellular biology of somatostatin receptors. Neuropeptides 35:1-23.

Csaba Z, Lelouvier B, Viollet C, El Ghouzzi V, Toyama K, Videau C, Bernard V and Dournaud P (2007) Activated somatostatin type 2 receptors traffic in vivo in central neurons from dendrites to the trans Golgi before recycling. Traffic 8: 820-834.

Csaba Z, Peineau S, and Dournaud P (2012) Molecular mechanisms of somatostatin receptor trafficking. J Mol Endocrinol 48:R1-R12.

Csaba Z, Pirker S, Lelouvier B, Simon A, Videau C, Epelbaum J, Czech T, Baumgartner C, Sperk G, and Dournaud P (2005) Somatostatin receptor type 2 undergoes plastic changes in the human epileptic dentate gyrus. J Neuropathol Exp Neurol 64:956-969. 
Csaba Z, Richichi C, Bernard V, Epelbaum J, Vezzani A, and Dournaud P (2004) Plasticity of somatostatin and somatostatin sst2A receptors in the rat dentate gyrus during kindling epileptogenesis. Eur J Neurosci 19:2531-2538.

Csaba Z, Simon A, Helboe L, Epelbaum J, and Dournaud P (2003) Targeting sst2A receptor-expressing cells in the rat hypothalamus through in vivo agonist stimulation: neuroanatomical evidence for a major role of this subtype in mediating somatostatin functions. Endocrinology 144:1564-1573.

Cuevas-Ramos D, Carmichael JD, Cooper O, Bonert VS, Gertych A, Mamelak AN, and Melmed S (2015) A structural and functional acromegaly classification. J Clin Endocrinol Metab 100:122-131.

Dal Monte M, Martini D, Ristori C, Azara D, Armani C, Balbarini A, and Bagnoli P (2011) Hypoxia effects on proangiogenic factors in human umbilical vein endothelial cells: functional role of the peptide somatostatin. Naunyn Schmiedebergs Arch Pharmacol 383:593-612.

Dal Monte M, Petrucci C, Vasilaki A, Cervia D, Grouselle D, Epelbaum J, Kreienkamp HJ, Richter D, Hoyer D, and Bagnoli P (2003) Genetic deletion of somatostatin receptor 1 alters somatostatinergic transmission in the mouse retina. Neuropharmacology 45:1080-1092.

Dalm VA, Hofland LJ, Mooy CM, Waaijers MA, van Koetsveld PM, Langerak AW, Staal FT, van der Lely AJ, Lamberts SW, and van Hagen MP (2004) Somatostatin receptors in malignant lymphomas: targets for radiotherapy? $\mathrm{J} \mathrm{Nucl} \mathrm{Med} \mathrm{45:8-16.}$

Dalm VA, van Hagen PM, van Koetsveld PM, Langerak AW, van der Lely A-J, Lamberts SW, and Hofland LJ (2003a) Cortistatin rather than somatostatin as a potential endogenous ligand for somatostatin receptors in the human immune system. J Clin Endocrinol Metab 88:270-276.

Dalm VASH, van Hagen PM, van Koetsveld PM, Achilefu S, Houtsmuller AB, Pols DHJ, van der Lely A-J, Lamberts SWJ, and Hofland LJ (2003b) Expression of somatostatin, cortistatin, and somatostatin receptors in human monocytes, macrophages, and dendritic cells. Am J Physiol Endocrinol Metab 285:E344-E353.

Daly AF, Tichomirowa MA, Petrossians P, Heliövaara E, Jaffrain-Rea ML, Barlier A Naves LA, Ebeling T, Karhu A, Raappana A, et al. (2010) Clinical characteristics and therapeutic responses in patients with germ-line AIP mutations and pituitary adenomas: an international collaborative study. J Clin Endocrinol Metab 95: E373-E383.

Day R, Dong W, Panetta R, Kraicer J, Greenwood MT, and Patel YC (1995) Expression of mRNA for somatostatin receptor (sstr) types 2 and 5 in individual rat pituitary cells: a double labeling in situ hybridization analysis. Endocrinology 136 5232-5235.

de Bruin C, Pereira AM, Feelders RA, Romijn JA, Roelfsema F, Sprij-Mooij DM, van Aken MO, van der Lelij AJ, de Herder WW, Lamberts SW, et al. (2009) Coexpression of dopamine and somatostatin receptor subtypes in corticotroph adenomas. J Clin Endocrinol Metab 94:1118-1124.

De Bundel D, Fafouri A, Csaba Z, Loyens E, Lebon S, El Ghouzzi V, Peineau S, Vodjdani G, Kiagiadaki F, Aourz N, et al. (2015) Trans-modulation of the somatostatin type $2 \mathrm{~A}$ receptor trafficking by insulin-regulated aminopeptidase decreases limbic seizures. J Neurosci 35:11960-11975.

de Lecea L (2008) Cortistatin-functions in the central nervous system. Mol Cell Endocrinol 286:88-95.

de Lecea L and Castaño JP (2006) Cortistatin: not just another somatostatin analog. Nat Clin Pract Endocrinol Metab 2:356-357.

de Lecea L, Criado JR, Prospero-Garcia O, Gautvik KM, Schweitzer P, Danielson PE, Dunlop CLM, Siggins GR, Henriksen SJ, and Sutcliffe JG (1996) A cortical neuropeptide with neuronal depressant and sleep-modulating properties. Nature 381:242-245.

de Lecea L, del Rio JA, Criado JR, Alcántara S, Morales M, Danielson PE, Henriksen SJ, Soriano E, and Sutcliffe JG (1997a) Cortistatin is expressed in a distinct subset of cortical interneurons. $J$ Neurosci 17:5868-5880.

de Lecea L, Ruiz-Lozano P, Danielson PE, Peelle-Kirley J, Foye PE, Frankel WN, and Sutcliffe JG (1997b) Cloning, mRNA expression, and chromosomal mapping of mouse and human preprocortistatin. Genomics 42:499-506.

Delesque N, Buscail L, Estève JP, Saint-Laurent N, Müller C, Weckbecker G, Bruns C, Vaysse N, and Susini C (1997) sst2 somatostatin receptor expression reverses tumorigenicity of human pancreatic cancer cells. Cancer Res 57:956-962.

Delgado-Maroto V, Benitez R, Forte-Lago I, Morell M, Maganto-Garcia E, SouzaMoreira L, O’Valle F, Durán-Prado M, Lichtman AH, Gonzalez-Rey E, et al. (2017) Cortistatin reduces atherosclerosis in hyperlipidemic ApoE-deficient mice and the formation of foam cells. Sci Rep 7:46444.

Demchyshyn LL, Srikant CB, Sunahara RK, Kent G, Seeman P, Van Tol HH, Panetta R, Patel YC, and Niznik HB (1993) Cloning and expression of a human somatostatin-14-selective receptor variant (somatostatin receptor 4) located on chromosome 20. Mol Pharmacol 43:894-901.

Deng QQ, Sheng WL, Zhang G, Weng SJ, Yang XL, and Zhong YM (2016) Signalling mechanism for somatostatin receptor 5-mediated suppression of AMPA responses in rat retinal ganglion cells. Neuropharmacology 107:215-226.

Dent P, Wang Y, Gu Y-Z, Wood SL, Reardon DB, Mangues R, Pellicer A, Schonbrunn A, and Sturgill TW (1997) S49 cells endogenously express subtype 2 somatostatin receptors which couple to increase protein tyrosine phosphatase activity in membranes and down-regulate Raf-1 activity in situ. Cell Signal 9:539-549.

Diakatou E, Alexandraki KI, Tsolakis AV, Kontogeorgos G, Chatzellis E, Leonti A, and Kaltsas GA (2015) Somatostatin and dopamine receptor expression in neuroendocrine neoplasms: correlation of immunohistochemical findings with somatostatin receptor scintigraphy visual scores. Clin Endocrinol (Oxf) 83:420-428.

Dolais-Kitabgi J, Kitabgi P, Brazeau P, and Freychet P (1979) Effect of neurotensin on insulin, glucagon, and somatostatin release from isolated pancreatic islets. Endocrinology 105:256-260.

Dörflinger U, Pscherer A, Moser M, Rümmele P, Schüle R, and Buettner R (1999) Activation of somatostatin receptor II expression by transcription factors MIBP1 and SEF-2 in the murine brain. Mol Cell Biol 19:3736-3747.

Dournaud P, Boudin H, Schonbrunn A, Tannenbaum GS, and Beaudet A (1998) Interrelationships between somatostatin sst2A receptors and somatostatincontaining axons in rat brain: evidence for regulation of cell surface receptors by endogenous somatostatin. J Neurosci 18:1056-1071.
Dournaud P, Gu YZ, Schonbrunn A, Mazella J, Tannenbaum GS, and Beaudet A (1996) Localization of the somatostatin receptor SST2A in rat brain using a specific anti-peptide antibody. J Neurosci 16:4468-4478.

Dournaud P, Slama A, Beaudet A, and Epelbaum J (2000) Somatostatin receptors, in Handbook of Chemical Neuroanatomy (Quirion R, Björklund A, and Hökfelt T eds) pp 1-43, Elsevier, Amsterdam, Netherlands.

Druckenthaner M, Schwarzer C, Ensinger C, Gabriel M, Prommegger R, Riccabona G, and Decristoforo C (2007) Evidence for somatostatin receptor 2 in thyroid tissue. Regul Pept 138:32-39.

Duerson K, White RE, Jiang F, Schonbrunn A, and Armstrong DL (1996) Somatostatin stimulates BKCa channels in rat pituitary tumor cells through lipoxygenase metabolites of arachidonic acid. Neuropharmacology 35:949-961.

Dufourny L, Delmas O, Teixeira-Gomes AP, Decourt C, and Sliwowska JH (2018) Neuroanatomical connections between kisspeptin neurones and somatostatin neurones in female and male rat hypothalamus: a possible involvement of SSTR1 in kisspeptin release. $J$ Neuroendocrinol 30:e12593.

Duluc C, Moatassim-Billah S, Chalabi-Dchar M, Perraud A, Samain R, Breibach F Gayral M, Cordelier P, Delisle MB, Bousquet-Dubouch MP, et al. (2015) Pharmacological targeting of the protein synthesis mTOR/4E-BP1 pathway in cancerassociated fibroblasts abrogates pancreatic tumour chemoresistance. EMBO Mol Med 7:735-753.

Durán-Prado M, Bucharles C, Gonzalez BJ, Vázquez-Martínez R, Martínez-Fuentes AJ, García-Navarro S, Rhodes SJ, Vaudry H, Malagón MM, and Castaño JP (2007) Porcine somatostatin receptor 2 displays typical pharmacological sst2 features but unique dynamics of homodimerization and internalization. Endocrinology 148 411-421.

Durán-Prado M, Gahete MD, Delgado-Niebla E, Martínez-Fuentes AJ, VázquezMartínez R, García-Navarro S, Gracia-Navarro F, Malagon MM, Luque RM, and Castaño JP (2012a) Truncated variants of pig somatostatin receptor subtype 5 (sst5) act as dominant-negative modulators for sst2-mediated signaling. Am $J$ Physiol Endocrinol Metab 303:E1325-E1334.

Durán-Prado M, Gahete MD, Hergueta-Redondo M, Martínez-Fuentes AJ, CórdobaChacón J, Palacios J, Gracia-Navarro F, Moreno-Bueno G, Malagón MM, Luque $\mathrm{RM}$, et al. (2012b) The new truncated somatostatin receptor variant sst5TMD4 is associated to poor prognosis in breast cancer and increases malignancy in MCF-7 cells. Oncogene 31:2049-2061.

Durán-Prado M, Gahete MD, Martínez-Fuentes AJ, Luque RM, Quintero A, Webb SM, Benito-López P, Leal A, Schulz S, Gracia-Navarro F, et al. (2009) Identification and characterization of two novel truncated but functional isoforms of the somatostatin receptor subtype 5 differentially present in pituitary tumors. $J$ Clin Endocrinol Metab 94:2634-2643.

Durán-Prado M, Malagón MM, Gracia-Navarro F, and Castaño JP (2008) Dimerization of $\mathrm{G}$ protein-coupled receptors: new avenues for somatostatin receptor signalling, control and functioning. Mol Cell Endocrinol 286:63-68.

Durán-Prado M, Morell M, Delgado-Maroto V, Castaño JP, Aneiros-Fernandez J, de Lecea L, Culler MD, Hernandez-Cortes P, O'Valle F, and Delgado M (2013) Cortistatin inhibits migration and proliferation of human vascular smooth muscle cells and decreases neointimal formation on carotid artery ligation. Circ Res 112 $1444-1455$

Durán-Prado M, Saveanu A, Luque RM, Gahete MD, Gracia-Navarro F, Jaquet P, Dufour H, Malagón MM, Culler MD, Barlier A, et al. (2010) A potential inhibitory role for the new truncated variant of somatostatin receptor 5, sst5TMD4, in pituitary adenomas poorly responsive to somatostatin analogs. J Clin Endocrinol Metab 95:2497-2502.

Efstathiadou ZA, Bargiota A, Chrisoulidou A, Kanakis G, Papanastasiou L, Theodoropoulou A, Tigas SK, Vassiliadi DA, Alevizaki M, and Tsagarakis S (2015) Impact of gsp mutations in somatotroph pituitary adenomas on growth hormone response to somatostatin analogs: a meta-analysis. Pituitary 18:861-867.

Eigler T and Ben-Shlomo A (2014) Somatostatin system: molecular mechanisms regulating anterior pituitary hormones. J Mol Endocrinol 53:R1-R19.

Eigler T, Ben-Shlomo A, Zhou C, Khalafi R, Ren S-G, and Melmed S (2014) Constitutive somatostatin receptor subtype-3 signaling suppresses growth hormone synthesis. Mol Endocrinol 28:554-564.

Einstein EB, Patterson CA, Hon BJ, Regan KA, Reddi J, Melnikoff DE, Mateer MJ, Schulz S, Johnson BN, and Tallent MK (2010) Somatostatin signaling in neuronal cilia is critical for object recognition memory. $J$ Neurosci 30:4306-4314.

Elberg G, Hipkin RW, and Schonbrunn A (2002) Homologous and heterologous regulation of somatostatin receptor 2. Mol Endocrinol 16:2502-2514.

Elekes K, Helyes Z, Kereskai L, Sándor K, Pintér E, Pozsgai G, Tékus V, Bánvölgyi A Németh J, Szüts T, et al. (2008) Inhibitory effects of synthetic somatostatin receptor subtype 4 agonists on acute and chronic airway inflammation and hyperreactivity in the mouse. Eur J Pharmacol 578:313-322.

Elston MS, Meyer-Rochow GY, Conaglen HM, Clarkson A, Clifton-Bligh RJ, Conaglen JV, and Gill AJ (2015) Increased SSTR2A and SSTR3 expression in succinate dehydrogenase-deficient pheochromocytomas and paragangliomas. Hum Pathol 46:390-396.

Engin E, Stellbrink J, Treit D, and Dickson CT (2008) Anxiolytic and antidepressant effects of intracerebroventricularly administered somatostatin: behavioral and neurophysiological evidence. Neuroscience 157:666-676.

Engin E and Treit D (2009) Anxiolytic and antidepressant actions of somatostatin the role of sst2 and sst3 receptors. Psychopharmacology (Berl) 206:281-289.

Engström M, Savola J-M, and Wurster S (2006) Differential efficacies of somatostatin receptor agonists for G-protein activation and desensitization of somatostatin receptor subtype 4-mediated responses. J Pharmacol Exp Ther 316:1262-1268.

Engström M, Tomperi J, El-Darwish K, Åhman M, Savola J-M, and Wurster S (2005) Superagonism at the human somatostatin receptor subtype 4. J Pharmacol Exp Ther 312:332-338.

Ensinck JW, Baskin DG, Vahl TP, Vogel RE, Laschansky EC, Francis BH, Hoffman RC, Krakover JD, Stamm MR, Low MJ, et al. (2002) Thrittene, homologous with somatostatin-28((1-13)), is a novel peptide in mammalian gut and circulation. Endocrinology 143:2599-2609. 
Ensinck JW, Laschansky EC, Vogel RE, and D'Alessio DA (2003) Effect of ingested nutrients on the release of thrittene into the human circulation. $J$ Clin Endocrinol Metab 88:4798-4804.

Epelbaum J, Dournaud P, Fodor M, and Viollet C (1994) The neurobiology of somatostatin. Crit Rev Neurobiol 8:25-44.

Esch F, Böhlen P, Ling N, Benoit R, Brazeau P, and Guillemin R (1980) Primary structure of ovine hypothalamic somatostatin-28 and somatostatin-25. Proc Natl Acad Sci USA 77:6827-6831.

Evangelou I, Petraki C, Msaouel P, Scorilas A, Sdrolia E, Padazi G, Koborozos V, and Koutsilieris M (2012) Immunohistochemical expression of somatostatin receptor subtypes 2 and 5 in colorectal cancer. Eur J Clin Invest 42:777-783.

Ezzat S, Snyder PJ, Young WF, Boyajy LD, Newman C, Klibanski A, Molitch ME, Boyd AE, Sheeler L, Cook DM, et al. (1992) Octreotide treatment of acromegaly: a randomized, multicenter study. Ann Intern Med 117:711-718.

Fani M, Braun F, Waser B, Beetschen K, Cescato R, Erchegyi J, Rivier JE, Weber WA, Maecke HR, and Reubi JC (2012) Unexpected sensitivity of sst2 antagonists to N-terminal radiometal modifications. J Nucl Med 53:1481-1489.

Fani M, Mueller A, Tamma M-L, Nicolas G, Rink HR, Cescato R, Reubi JC, and Maecke HR (2010) Radiolabeled bicyclic somatostatin-based analogs: a novel class of potential radiotracers for SPECT/PET of neuroendocrine tumors. $J$ Nucl Med 51:1771-1779.

Farb TB, Adeva M, Beauchamp TJ, Cabrera O, Coates DA, Meredith TD, Droz BA, Efanov A, Ficorilli JV, Gackenheimer SL, et al. (2017) Regulation of endogenous (male) rodent GLP-1 secretion and human islet insulin secretion by antagonism of somatostatin receptor 5. Endocrinology 158:3859-3873.

Farrell SR, Rankin DR, Brecha NC, and Barnes S (2014) Somatostatin receptor subtype 4 modulates L-type calcium channels via G $\beta \gamma$ and $\mathrm{PKC}$ signaling in rat retinal ganglion cells. Channels (Austin) 8:519-527.

Farrell SR, Raymond ID, Foote M, Brecha NC, and Barnes S (2010) Modulation of voltage-gated ion channels in rat retinal ganglion cells mediated by somatostatin receptor subtype 4. J Neurophysiol 104:1347-1354

Fee C, Banasr M, and Sibille E (2017) Somatostatin-positive gamma-aminobutyric acid interneuron deficits in depression: cortical microcircuit and therapeutic perspectives. Biol Psychiatry 82:549-559.

Fehlmann D, Langenegger D, Schuepbach E, Siehler S, Feuerbach D, and Hoyer D (2000) Distribution and characterisation of somatostatin receptor mRNA and binding sites in the brain and periphery. J Physiol Paris 94:265-281.

Ferjoux G, Lopez F, Esteve J-P, Ferrand A, Vivier E, Vely F, Saint-Laurent N, Pradayrol L, Buscail L, and Susini C (2003) Critical role of Src and SHP-2 in sst2 somatostatin receptor-mediated activation of SHP-1 and inhibition of cell proliferation. Mol Biol Cell 14:3911-3928.

Ferone D, Pivonello R, Van Hagen PM, Dalm VA, Lichtenauer-Kaligis EG, Waaijers M, Van Koetsveld PM, Mooy DM, Colao A, Minuto F, et al. (2002) Quantitative and functional expression of somatostatin receptor subtypes in human thymocytes. Am $J$ Physiol Endocrinol Metab 283:E1056-E1066.

Ferone D, van Hagen MP, Kwekkeboom DJ, van Koetsveld PM, Mooy DM, Lichtenauer-Kaligis E, Schönbrunn A, Colao A, Lamberts SWJ, and Hofland LJ (2000) Somatostatin receptor subtypes in human thymoma and inhibition of cell proliferation by octreotide in vitro. J Clin Endocrinol Metab 85:1719-1726.

Ferone D, van Hagen PM, van Koetsveld PM, Zuijderwijk J, Mooy DM, LichtenauerKaligis EG, Colao A, Bogers AJ, Lombardi G, Lamberts SW, et al. (1999) In vitro characterization of somatostatin receptors in the human thymus and effects of somatostatin and octreotide on cultured thymic epithelial cells. Endocrinology 140: 373-380.

Ferrante E, Pellegrini C, Bondioni S, Peverelli E, Locatelli M, Gelmini P, Luciani P, Peri A, Mantovani G, Bosari S, et al. (2006) Octreotide promotes apoptosis in human somatotroph tumor cells by activating somatostatin receptor type 2 . Endocr Relat Cancer 13:955-962.

Feuerbach D, Fehlmann D, Nunn C, Siehler S, Langenegger D, Bouhelal R, Seuwen $\mathrm{K}$, and Hoyer D (2000) Cloning, expression and pharmacological characterisation of the mouse somatostatin sst(5) receptor. Neuropharmacology 39:1451-1462.

Fischer T, Doll C, Jacobs S, Kolodziej A, Stumm R, and Schulz S (2008) Reassessment of sst2 somatostatin receptor expression in human normal and neoplastic tissues using the novel rabbit monoclonal antibody UMB-1. J Clin Endocrinol Metab 93:4519-4524.

Fliers E, van Furth WR, and Bisschop PH (2012) Cure of a thyrotrophin (TSH)secreting pituitary adenoma by medical therapy. Clin Endocrinol (Oxf) 77: $788-790$.

Fløgstad AK, Halse J, Bakke S, Lancranjan I, Marbach P, Bruns C, and Jervell J (1997) Sandostatin LAR in acromegalic patients: long-term treatment. $J$ Clin Endocrinol Metab 82:23-28.

Fløgstad AK, Halse J, Grass P, Abisch E, Djøseland O, Kutz K, Bodd E, and Jervell J (1994) A comparison of octreotide, bromocriptine, or a combination of both drugs in acromegaly. J Clin Endocrinol Metab 79:461-465.

Florio T, Barbieri F, Spaziante R, Zona G, Hofland LJ, van Koetsveld PM, Feelders RA, Stalla GK, Theodoropoulou M, Culler MD, et al. (2008) Efficacy of a dopaminesomatostatin chimeric molecule, BIM-23A760, in the control of cell growth from primary cultures of human non-functioning pituitary adenomas: a multi-center study. Endocr Relat Cancer 15:583-596.

Florio T, Rim C, Hershberger RE, Loda M, and Stork PJ (1994) The somatostatin receptor SSTR1 is coupled to phosphotyrosine phosphatase activity in CHO-K1 cells. Mol Endocrinol 8:1289-1297.

Florio T, Scorizello A, Fattore M, D'Alto V, Salzano S, Rossi G, Berlingieri MT, Fusco A and Schettini G (1996) Somatostatin inhibits PC Cl3 thyroid cell proliferation through the modulation of phosphotyrosine activity: impairment of the somatostatinergic ef fects by stable expression of E1A viral oncogene. J Biol Chem 271:6129-6136.

Florio T, Yao H, Carey KD, Dillon TJ, and Stork PJ (1999) Somatostatin activation of mitogen-activated protein kinase via somatostatin receptor 1 (SSTR1). Mol Endocrinol 13:24-37.

Fodor M, van Elk EJ, Huizinga CT, Prins T, and Delemarre-van de Waal HA (2005) NPY neurons express somatostatin receptor subtype 1 in the arcuate nucleus. Neuroreport 16:29-32.
Fougner SL, Bollerslev J, Latif F, Hald JK, Lund T, Ramm-Pettersen J, and Berg JP (2008a) Low levels of raf kinase inhibitory protein in growth hormone-secreting pituitary adenomas correlate with poor response to octreotide treatment. $J$ Clin Endocrinol Metab 93:1211-1216.

Fougner SL, Bollerslev J, Svartberg J, Øksnes M, Cooper J, and Carlsen SM (2014) Preoperative octreotide treatment of acromegaly: long-term results of a randomised controlled trial. Eur J Endocrinol 171:229-235.

Fougner SL, Borota OC, Berg JP, Hald JK, Ramm-Pettersen J, and Bollerslev J (2008b) The clinical response to somatostatin analogues in acromegaly correlates to the somatostatin receptor subtype 2 a protein expression of the adenoma. Clin Endocrinol $(\mathrm{Oxf})$ 68:458-465.

Fougner SL, Casar-Borota O, Heck A, Berg JP, and Bollerslev J (2012) Adenoma granulation pattern correlates with clinical variables and effect of somatostatin analogue treatment in a large series of patients with acromegaly. Clin Endocrinol (Oxf) 76:96-102.

Fougner SL, Lekva T, Borota OC, Hald JK, Bollerslev J, and Berg JP (2010) The expression of E-cadherin in somatotroph pituitary adenomas is related to tumor size, invasiveness, and somatostatin analog response. J Clin Endocrinol Metab $\mathbf{9 5}$ 2334-2342.

Frati A, Rouzier R, Lesieur B, Werkoff G, Antoine M, Rodenas A, Darai E, and Chereau E (2014) Expression of somatostatin type-2 and -4 receptor and correlation with histological type in breast cancer. Anticancer Res 34:3997-4003.

Freda PU, Katznelson L, van der Lely AJ, Reyes CM, Zhao S, and Rabinowitz D (2005) Long-acting somatostatin analog therapy of acromegaly: a meta-analysis. $J$ Clin Endocrinol Metab 90:4465-4473.

Fruhwald MC, Rickert CH, O'Dorisio MS, Madsen M, Warmuth-Metz M, Khanna G, Paulus W, Kuhl J, Jurgens H, Schneider P, et al. (2004) Somatostatin receptor subtype 2 is expressed by supratentorial primitive neuroectodermal tumors of childhood and can be targeted for somatostatin receptor imaging. Clin Cancer Res 10:2997-3006.

Gabalec F, Drastikova M, Cesak T, Netuka D, Masopust V, Machac J, Marek J, Cap $\mathrm{J}$, and Beranek M (2015) Dopamine 2 and somatostatin 1-5 receptors coexpression in clinically non-functioning pituitary adenomas. Physiol Res 64:369-377.

Gabriel M, Decristoforo C, Donnemiller E, Ulmer H, Watfah Rychlinski C, Mather SJ, and Moncayo R (2003) An intrapatient comparison of $99 \mathrm{mTc}-\mathrm{EDDA} / \mathrm{HYNIC}$ TOC with 111In-DTPA-octreotide for diagnosis of somatostatin receptorexpressing tumors. J Nucl Med 44:708-716.

Gadelha MR, Bronstein MD, Brue T, Coculescu M, Fleseriu M, Guitelman M, Pronin V, Raverot G, Shimon I, Lievre KK, et al.; Pasireotide C2402 Study Group (2014) Pasireotide versus continued treatment with octreotide or lanreotide in patients with inadequately controlled acromegaly (PAOLA): a randomised, phase 3 trial. Lancet Diabetes Endocrinol 2:875-884.

Gadelha MR, Kasuki L, and Korbonits M (2013) Novel pathway for somatostatin analogs in patients with acromegaly. Trends Endocrinol Metab 24:238-246.

Gadelha MR, Wildemberg LE, Bronstein MD, Gatto F, and Ferone D (2017) Somatostatin receptor ligands in the treatment of acromegaly. Pituitary 20:100-108

Gahete MD, Cordoba-Chacón J, Durán-Prado M, Malagón MM, Martinez-Fuentes AJ, Gracia-Navarro F, Luque RM, and Castaño JP (2010a) Somatostatin and its receptors from fish to mammals. Ann N Y Acad Sci 1200:43-52.

Gahete MD, Durán-Prado M, Delgado-Niebla E, Garrido JJ, Rhodes SJ, GarcíaNavarro S, Gracia-Navarro F, Malagón MM, Luque RM, and Castaño JP (2014) Porcine sst1 can physically interact with other somatostatin receptors, and its expression is regulated by metabolic/inflammatory sensors. Am J Physiol Endocrinol Metab 306:E483-E493.

Gahete MD, Durán-Prado M, Luque RM, Martínez-Fuentes AJ, Vázquez-Martínez R, Malagón MM, and Castaño JP (2008) Are somatostatin and cortistatin two siblings in regulating endocrine secretions? In vitro work ahead. Mol Cell Endocrinol $\mathbf{2 8 6}$ 128-134.

Gahete MD, Rincón-Fernández D, Durán-Prado M, Hergueta-Redondo M, IbáñezCosta A, Rojo-Sebastián A, Gracia-Navarro F, Culler MD, Casanovas O, MorenoBueno G et al (2016) The truncated somatostatin receptor sst5TMD4 stimulates the angiogenic process and is associated to lymphatic metastasis and disease-free survival in breast cancer patients. Oncotarget 7:60110-60122.

Gahete MD, Rubio A, Durán-Prado M, Avila J, Luque RM, and Castaño JP (2010b) Expression of somatostatin, cortistatin, and their receptors, as well as dopamine receptors, but not of neprilysin, are reduced in the temporal lobe of Alzheimer's disease patients. J Alzheimers Dis 20:465-475.

Garcia PD and Myers RM (1994) Pituitary cell line GH3 expresses two somatostatin receptor subtypes that inhibit adenylyl cyclase: functional expression of rat somatostatin receptor subtypes 1 and 2 in human embryonic kidney 293 cells. $M o$ Pharmacol 45:402-409.

Gardair C, Samimi M, Touzé A, Coursaget P, Lorette G, Caille A, Wierzbicka E, Croué A, Avenel-Audran M, Aubin F, et al. (2015) Somatostatin receptors $2 \mathrm{~A}$ and 5 are expressed in merkel cell carcinoma with no association with disease severity. Neuroendocrinology 101:223-235.

Gastambide F, Lepousez G, Viollet C, Loudes C, Epelbaum J, and Guillou J-L (2010) Cooperation between hippocampal somatostatin receptor subtypes 4 and 2: functional relevance in interactive memory systems. Hippocampus 20:745-757.

Gatto F, Barbieri F, Gatti M, Wurth R, Schulz S, Ravetti JL, Zona G, Culler MD, Saveanu A, Giusti M, et al. (2012) Balance between somatostatin and D2 receptor expression drives TSH-secreting adenoma response to somatostatin analogues and dopastatins. Clin Endocrinol (Oxf) 76:407-414.

Gatto F, Feelders R, van der Pas R, Kros JM, Dogan F, van Koetsveld PM, van der Lelij A-J, Neggers SJCMM, Minuto F, de Herder W, et al. (2013a) $\beta$-Arrestin 1 and 2 and $\mathrm{G}$ protein-coupled receptor kinase 2 expression in pituitary adenomas: role in the regulation of response to somatostatin analogue treatment in patients with acromegaly. Endocrinology 154:4715-4725.

Gatto F, Feelders RA, Franck SE, van Koetsveld PM, Dogan F, Kros JM, Neggers SJCMM, van der Lely A-J, Lamberts SWJ, Ferone D, et al. (2017) In vitro head-tohead comparison between octreotide and pasireotide in GH-secreting pituitary adenomas. J Clin Endocrinol Metab 102:2009-2018. 
Gatto F, Feelders RA, van der Pas R, Kros JM, Waaijers M, Sprij-Mooij D, Neggers SJ, van der Lelij AJ, Minuto F, Lamberts SW, et al. (2013b) Immunoreactivity score using an anti-sst2A receptor monoclonal antibody strongly predicts the biochemical response to adjuvant treatment with somatostatin analogs in acromegaly. $J$ Clin Endocrinol Metab 98:E66-E71.

Geneva II, Tan HY, and Calvert PD (2017) Untangling ciliary access and enrichment of two rhodopsin-like receptors using quantitative fluorescence microscopy reveals cell-specific sorting pathways. Mol Biol Cell 28:554-566.

Ginj M, Chen J, Walter MA, Eltschinger V, Reubi JC, and Maecke HR (2005) Preclinical evaluation of new and highly potent analogues of octreotide for predictive imaging and targeted radiotherapy. Clin Cancer Res 11:1136-1145.

Ginj M, Schmitt JS, Chen J, Waser B, Reubi J-C, de Jong M, Schulz S, and Maecke HR (2006a) Design, synthesis, and biological evaluation of somatostatin-based radiopeptides. Chem Biol 13:1081-1090.

Ginj M, Zhang H, Eisenwiener K-P, Wild D, Schulz S, Rink H, Cescato R, Reubi JC and Maecke HR (2008) New pansomatostatin ligands and their chelated versions: affinity profile, agonist activity, internalization, and tumor targeting. Clin Cancer Res 14:2019-2027.

Ginj M, Zhang H, Waser B, Cescato R, Wild D, Wang X, Erchegyi J, Rivier J, Mäcke HR, and Reubi JC (2006b) Radiolabeled somatostatin receptor antagonists are preferable to agonists for in vivo peptide receptor targeting of tumors. Proc Natl Acad Sci USA 103:16436-16441.

Giustina A, Bevan JS, Bronstein MD, Casanueva FF, Chanson P, Petersenn S, Thanh X-MT, Sert C, Houchard A, Guillemin I, et al.; SAGIT Investigator Group (2016) SAGIT®: clinician-reported outcome instrument for managing acromegaly in clinical practice-development and results from a pilot study. Pituitary 19:39-49.

Giustina A, Chanson P, Kleinberg D, Bronstein MD, Clemmons DR, Klibanski A, van der Lely AJ, Strasburger CJ, Lamberts SW, Ho KK, et al.; Acromegaly Consensus Group (2014) Expert consensus document: a consensus on the medical treatment of acromegaly. Nat Rev Endocrinol 10:243-248.

Giustina A, Mazziotti G, Torri V, Spinello M, Floriani I, and Melmed S (2012) Metaanalysis on the effects of octreotide on tumor mass in acromegaly. PLoS One 7 e36411.

Gonzalez-Rey E, Chorny A, Del Moral RG, Varela N, and Delgado M (2007) Therapeutic effect of cortistatin on experimental arthritis by downregulating in flammatory and Th1 responses. Ann Rheum Dis 66:582-588.

Gonzalez-Rey E, Chorny A, Robledo G, and Delgado M (2006a) Cortistatin, a new antiinflammatory peptide with therapeutic effect on lethal endotoxemia. $J$ Exp Med 203:563-571.

Gonzalez-Rey E, Pedreño M, Delgado-Maroto V, Souza-Moreira L, and Delgado M (2015) Lulling immunity, pain, and stress to sleep with cortistatin. Ann N Y Acad Sci 1351:89-98.

Gonzalez-Rey E, Varela N, Sheibanie AF, Chorny A, Ganea D, and Delgado M (2006b) Cortistatin, an antiinflammatory peptide with therapeutic action in inflammatory bowel disease. Proc Natl Acad Sci USA 103:4228-4233.

Gordon DF, Woodmansee WW, Lewis SR, James RA, Wood WM, and Ridgway EC (1999) Cloning of the mouse somatostatin receptor subtype 5 gene: promoter structure and function. Endocrinology 140:5598-5608.

Gorham L, Just S, and Doods H (2014a) Somatostatin 4 receptor activation modulates G-protein coupled inward rectifying potassium channels and voltage stimulated calcium signals in dorsal root ganglion neurons. Eur $J$ Pharmacol $\mathbf{7 3 6}$ 101-106.

Gorham L, Just S, and Doods H (2014b) Somatostatin 4 receptor activation modulates TRPV1[correction of TPRV1] currents in dorsal root ganglion neurons. Neurosci Lett 573:35-39.

Grant M, Alturaihi H, Jaquet P, Collier B, and Kumar U (2008) Cell growth inhibition and functioning of human somatostatin receptor type 2 are modulated by receptor heterodimerization. Mol Endocrinol 22:2278-2292.

Grant M, Patel RC, and Kumar U (2004) The role of subtype-specific ligand binding and the C-tail domain in dimer formation of human somatostatin receptors. $J$ Biol Chem 279:38636-38643.

Grant N, Clark D, Garsky V, Jaunakais I, McGregor W, and Sarantakis D (1976) Dissociation of somatostatin effects: peptides inhibiting the release of growth hormone but not glucagon or insulin in rats. Life Sci 19:629-631.

Green JA, Schmid CL, Bley E, Monsma PC, Brown A, Bohn LM, and Mykytyn K (2015) Recruitment of $\beta$-arrestin into neuronal cilia modulates somatostatin receptor subtype 3 ciliary localization. Mol Cell Biol 36:223-235.

Greenman Y and Melmed S (1994a) Expression of three somatostatin receptor subtypes in pituitary adenomas: evidence for preferential SSTR5 expression in the mammosomatotroph lineage. J Clin Endocrinol Metab 79:724-729.

Greenman Y and Melmed S (1994b) Heterogeneous expression of two somatostatin receptor subtypes in pituitary tumors. J Clin Endocrinol Metab 78:398-403.

Greenwood MT, Hukovic N, Kumar U, Panetta R, Hjorth SA, Srikant CB, and Patel YC (1997) Ligand binding pocket of the human somatostatin receptor 5: mutational analysis of the extracellular domains. Mol Pharmacol 52:807-814.

Greenwood MT, Panetta R, Robertson LA, Liu JL, and Patel YC (1994) Sequence analysis of the 5 '-flanking promoter region of the human somatostatin receptor 5 Biochem Biophys Res Commun 205:1883-1890.

Greenwood MT, Robertson LA, and Patel YC (1995) Cloning of the gene encoding human somatostatin receptor 2: sequence analysis of the 5 '-flanking promoter region. Gene 159:291-292.

Gromada J, Høy M, Buschard K, Salehi A, and Rorsman P (2001) Somatostatin inhibits exocytosis in rat pancreatic alpha-cells by G(i2)-dependent activation of calcineurin and depriming of secretory granules. J Physiol 535:519-532.

Gruszka A, Culler MD, and Melmed S (2012) Somatostatin analogs and chimeric somatostatin-dopamine molecules differentially regulate human growth hormone and prolactin gene expression and secretion in vitro. Mol Cell Endocrinol 362:104-109.

Gu F, Schumacher FR, Canzian F, Allen NE, Albanes D, Berg CD, Berndt SI, Boeing H, Bueno-de-Mesquita HB, Buring JE, et al. (2010) Eighteen insulin-like growth factor pathway genes, circulating levels of IGF-I and its binding protein, and risk of prostate and breast cancer. Cancer Epidemiol Biomarkers Prev 19:2877-2887.
Gu YZ, Brown PJ, Loose-Mitchell DS, Stork PJ, and Schonbrunn A (1995a) Development and use of a receptor antibody to characterize the interaction between somatostatin receptor subtype 1 and G proteins. Mol Pharmacol 48:1004-1014.

Gu YZ and Schonbrunn A (1997) Coupling specificity between somatostatin receptor sst2A and G proteins: isolation of the receptor-G protein complex with a receptor antibody. Mol Endocrinol 11:527-537.

Gu ZF, Corleto VD, Mantey SA, Coy DH, Maton PN, and Jensi RT (1995b) Somatostatin receptor subtype 3 mediates the inhibitory action of somatostatin on gastric smooth muscle cells. Am J Physiol 268:G739-G748.

Guadiana SM, Parker AK, Filho GF, Sequeira A, Semple-Rowland S, Shaw G, Mandel RJ, Foster TC, Kumar A, and Sarkisian MR (2016) Type 3 adenylyl cyclase and somatostatin receptor 3 expression persists in aged rat neocortical and hippocampal neuronal cilia. Front Aging Neurosci 8:127.

Gugger M, Waser B, Kappeler A, Schonbrunn A, and Reubi JC (2004) Cellular detection of sst2A receptors in human gastrointestinal tissue. Gut 53:1431-1436.

Guillermet J, Saint-Laurent N, Rochaix P, Cuvillier O, Levade T, Schally AV, Pradayrol L, Buscail L, Susini C, and Bousquet C (2003) Somatostatin receptor subtype 2 sensitizes human pancreatic cancer cells to death ligand-induced apoptosis. Proc Natl Acad Sci USA 100:155-160.

Günther T, Culler M, and Schulz S (2016) Research resource: real-time analysis of somatostatin and dopamine receptor signaling in pituitary cells using a fluorescence-based membrane potential assay. Mol Endocrinol 30:479-490.

Gurel MH, Han Y, Stevens AL, Furtado A, and Cox D (2017) Treatment adherence and persistence with long-acting somatostatin analog therapy for the treatment of acromegaly: a retrospective analysis. BMC Pharmacol Toxicol 18:22.

Guyotat J, Champier J, Pierre GS, Jouvet A, Bret P, Brisson C, Belin MF, Signorelli F, and Montange MF (2001) Differential expression of somatostatin receptors in medulloblastoma. J Neurooncol 51:93-103.

Hadcock JR, Strnad J, and Eppler CM (1994) Rat somatostatin receptor type 1 couples to G proteins and inhibition of cyclic AMP accumulation. Mol Pharmacol 45 $410-416$.

Hagiwara A, Inoue Y, Wakasa K, Haba T, Tashiro T, and Miyamoto T (2003) Comparison of growth hormone-producing and non-growth hormone-producing pituitary adenomas: imaging characteristics and pathologic correlation. Radiology 228: 533-538.

Hall GH, Turnbull LW, Richmond I, Helboe L, and Atkin SL (2002) Localisation of somatostatin and somatostatin receptors in benign and malignant ovarian tumours. Br J Cancer 87:86-90.

Hammond PJ, Wade AF, Gwilliam ME, Peters AM, Myers MJ, Gilbey SG, Bloom SR, and Calam J (1993) Amino acid infusion blocks renal tubular uptake of an indiumlabelled somatostatin analogue. Br J Cancer 67:1437-1439.

Händel M, Schulz S, Stanarius A, Schreff M, Erdtmann-Vourliotis M, Schmidt H, Wolf $G$, and Höllt V (1999) Selective targeting of somatostatin receptor 3 to neuronal cilia. Neuroscience 89:909-926.

Hannon JP, Petrucci C, Fehlmann D, Viollet C, Epelbaum J, and Hoyer D (2002) Somatostatin sst2 receptor knock-out mice: localisation of sst1-5 receptor mRNA and binding in mouse brain by semi-quantitative RT-PCR, in situ hybridisation histochemistry and receptor autoradiography. Neuropharmacology 42:396-413.

Hart MJ, Jiang X, Kozasa T, Roscoe W, Singer WD, Gilman AG, Sternweis PC, and Bollag G (1998) Direct stimulation of the guanine nucleotide exchange activity of p115 RhoGEF by Galpha13. Science 280:2112-2114.

Hayashi K, Inoshita N, Kawaguchi K, Ibrahim Ardisasmita A, Suzuki H, Fukuhara N, Okada M, Nishioka H, Takeuchi Y, Komada M, et al. (2016) The USP8 mutational status may predict drug susceptibility in corticotroph adenomas of Cushing's disease. Eur J Endocrinol 174:213-226.

He S, Dobbelaar PH, Guo L, Ye Z, Liu J, Jian T, Truong Q, Shah SK, Du W, Qi H, et al. (2016) SAR exploration at the C-3 position of tetrahydro- $\beta$-carboline sstr3 antagonists. Bioorg Med Chem Lett 26:1529-1535.

He S, Lai Z, Ye Z, Dobbelaar PH, Shah SK, Truong Q, Du W, Guo L, Liu J, Jian T, et al. (2014) Investigation of cardiovascular effects of tetrahydro- $\beta$-carboline sstr3 antagonists. ACS Med Chem Lett 5:748-753.

He S, Ye Z, Truong Q, Shah S, Du W, Guo L, Dobbelaar PH, Lai Z, Liu J, Jian T, et al. (2012) The discovery of MK-4256, a potent SSTR3 antagonist as a potential treatment of type 2 diabetes. ACS Med Chem Lett 3:484-489.

Heck A, Emblem KE, Casar-Borota O, Bollerslev J, and Ringstad G (2016a) Quantitative analyses of T2-weighted MRI as a potential marker for response to somatostatin analogs in newly diagnosed acromegaly. Endocrine 52:333-343.

Heck A, Emblem KE, Casar-Borota O, Ringstad G, and Bollerslev J (2016b) MRI T2 characteristics in somatotroph adenomas following somatostatin analog treatment in acromegaly. Endocrine 53:327-330.

Heiman ML, Murphy WA, and Coy DH (1987) Differential binding of somatostatin agonists to somatostatin receptors in brain and adenohypophysis. Neuroendocrinology 45:429-436.

Helboe L, Møller M, Nørregaard L, Schiødt M, and Stidsen CE (1997) Development of selective antibodies against the human somatostatin receptor subtypes sst1-sst5 Brain Res Mol Brain Res 49:82-88.

Helboe L, Stidsen CE, and Moller M (1998) Immunohistochemical and cytochemical localization of the somatostatin receptor subtype sst1 in the somatostatinergic parvocellular neuronal system of the rat hypothalamus. $J$ Neurosci 18:4938-4945.

Helyes Z, Pintér E, Sándor K, Elekes K, Bánvölgyi A, Keszthelyi D, Szőke E, Tóth DM, Sándor Z, Kereskai L, et al. (2009) Impaired defense mechanism against inflammation, hyperalgesia, and airway hyperreactivity in somatostatin 4 receptor gene-deleted mice. Proc Natl Acad Sci USA 106:13088-13093.

Hennigs JK, Müller J, Adam M, Spin JM, Riedel E, Graefen M, Bokemeyer C, Sauter G, Huland H, Schlomm T, et al. (2014) Loss of somatostatin receptor subtype 2 in prostate cancer is linked to an aggressive cancer phenotype, high tumor cell proliferation and predicts early metastatic and biochemical relapse. PLoS One $\mathbf{9}$ e100469.

Herac M, Niederle B, Raderer M, Krebs M, Kaserer K, and Koperek O (2016) Expression of somatostatin receptor $2 \mathrm{~A}$ in medullary thyroid carcinoma is associated with lymph node metastasis. APMIS 124:839-845. 
Hernández C and Simó R; European Consortium for the Early Treatment of Diabetic Retinopathy (EUROCONDOR) (2013) Somatostatin replacement: a new strategy for treating diabetic retinopathy. Curr Med Chem 20:3251-3257.

Hernández C, Simó-Servat O, and Simó R (2014) Somatostatin and diabetic retinopathy: current concepts and new therapeutic perspectives. Endocrine 46:209-214.

Herrera-Martinez AD, Gahete MD, Pedraza-Arevalo S, Sanchez-Sanchez R, OrtegaSalas R, Serrano-Blanch R, Luque RM, Galvez-Moreno MA and Castaño JP (2017a) Clinical and functional implication of the components of somatostatin system in gastroenteropancreatic neuroendocrine tumors. Endocrine 59:426-437.

Herrera-Martínez AD, Gahete MD, Sánchez-Sánchez R, Salas RO, Serrano-Blanch R, Salvatierra Á, Hofland LJ, Luque RM, Gálvez-Moreno MA, and Castaño JP (2017b) The components of somatostatin and ghrelin systems are altered in neuroendocrine lung carcinoids and associated to clinical-histological features. Lung Cancer 109:128-136.

Hershberger RE, Newman BL, Florio T, Bunzow J, Civelli O, Li XJ, Forte M, and Stork PJ (1994) The somatostatin receptors SSTR1 and SSTR2 are coupled to inhibition of adenylyl cyclase in Chinese hamster ovary cells via pertussis toxinsensitive pathways. Endocrinology 134:1277-1285.

Hervieu G and Emson PC (1998) The localization of somatostatin receptor 1 (sst1) immunoreactivity in the rat brain using an N-terminal specific antibody. Neuroscience 85:1263-1284.

Hicks RJ (2010) Use of molecular targeted agents for the diagnosis, staging and therapy of neuroendocrine malignancy. Cancer Imaging 10 Spec no A:S83-S91.

Hierowski MT, Liebow C, du Sapin K, and Schally AV (1985) Stimulation by somatostatin of dephosphorylation of membrane proteins in pancreatic cancer MIA PaCa-2 cell line. FEBS Lett 179:252-256.

Hipkin RW, Wang Y, and Schonbrunn A (2000) Protein kinase C activation stimulates the phosphorylation and internalization of the sst2A somatostatin receptor. J Biol Chem 275:5591-5599.

Hirose H, Yamasaki T, Ogino M, Mizojiri R, Tamura-Okano Y, Yashiro H, Muraki Y, Nakano Y, Sugama J, Hata A, et al. (2017) Discovery of novel 5-oxa-2,6diazaspiro[3.4]oct-6-ene derivatives as potent, selective, and orally available somatostatin receptor subtype 5 (SSTR5) antagonists for treatment of type 2 diabetes mellitus. Bioorg Med Chem 25:4175-4193.

Hocart SJ, Jain R, Murphy WA, Taylor JE, and Coy DH (1999) Highly potent cyclic disulfide antagonists of somatostatin. $J$ Med Chem 42:1863-1871.

Hocart SJ, Jain R, Murphy WA, Taylor JE, Morgan B, and Coy DH (1998) Potent antagonists of somatostatin: synthesis and biology. J Med Chem 41:1146-1154.

Hofland LJ and Lamberts SW (2003) The pathophysiological consequences of somatostatin receptor internalization and resistance. Endocr Rev 24:28-47.

Hofland LJ, Lamberts SWJ, and Feelders RA (2010) Role of somatostatin receptors in normal and tumoral pituitary corticotropic cells. Neuroendocrinology 92 (Suppl 1): $11-16$

Hofland LJ, Liu Q, Van Koetsveld PM, Zuijderwijk J, Van Der Ham F, De Krijger RR, Schonbrunn A, and Lamberts SW (1999) Immunohistochemical detection of somatostatin receptor subtypes sst1 and sst2A in human somatostatin receptor positive tumors. J Clin Endocrinol Metab 84:775-780.

Hofland LJ, van der Hoek J, Feelders R, van Aken MO, van Koetsveld PM, Waaijers M, Sprij-Mooij D, Bruns C, Weckbecker G, de Herder WW, et al. (2005) The multiligand somatostatin analogue SOM230 inhibits ACTH secretion by cultured human corticotroph adenomas via somatostatin receptor type 5. Eur J Endocrinol 152:645-654

Holliday ND, Tough IR, and Cox HM (2007) A functional comparison of recombinant and native somatostatin sst2 receptor variants in epithelia. $\mathrm{Br} J$ Pharmacol 152: 132-140.

Horiguchi K, Yamada M, Satoh T, Hashimoto K, Hirato J, Tosaka M, Yamada S, and Mori M (2009) Transcriptional activation of the mixed lineage leukemiap27Kip1 pathway by a somatostatin analogue. Clin Cancer Res 15:2620-2629.

Hormaechea-Agulla D, Jiménez-Vacas JM, Gómez-Gómez E, L-López F, CarrascoValiente J, Valero-Rosa J, Moreno MM, Sánchez-Sánchez R, Ortega-Salas R, Gracia-Navarro F, et al. (2017) The oncogenic role of the spliced somatostatin receptor sst5TMD4 variant in prostate cancer. FASEB J 31:4682-4696.

Hortala M, Ferjoux G, Estival A, Bertrand C, Schulz S, Pradayrol L, Susini C, and Clemente F (2003) Inhibitory role of the somatostatin receptor SST2 on the intracrine-regulated cell proliferation induced by the 210 -amino acid fibroblast growth factor-2 isoform: implication of JAK2. J Biol Chem 278:20574-20581.

Hou C, Gilbert RL, and Barber DL (1994) Subtype-specific signaling mechanisms of somatostatin receptors SSTR1 and SSTR2. J Biol Chem 269:10357-10362.

Hoyer D, Bell GI, Berelowitz M, Epelbaum J, Feniuk W, Humphrey PPA, O'Carroll AM, Patel YC, Schonbrunn A, Taylor JE, et al. (1995a) Classification and nomenclature of somatostatin receptors. Trends Pharmacol Sci 16:86-88.

Hoyer D, Nunn C, Hannon J, Schoeffter P, Feuerbach D, Schuepbach E, Langenegger D, Bouhelal R, Hurth K, Neumann P, et al. (2004) SRA880, in vitro characterization of the first non-peptide somatostatin sst(1) receptor antagonist. Neurosci Lett 361:132-135.

Hoyer D, Pérez J, Schoeffter P, Langenegger D, Schüpbach E, Kaupmann K, Lübbert H, Bruns C, and Reubi JC (1995b) Pharmacological identity between somatostatin SS-2 binding sites and SSTR-1 receptors. Eur J Pharmacol 289:151-161.

Hubina E, Nanzer AM, Hanson MR, Ciccarelli E, Losa M, Gaia D, Papotti M, Terren MR, Khalaf S, Jordan S, et al. (2006) Somatostatin analogues stimulate p27 expression and inhibit the MAP kinase pathway in pituitary tumours. Eur J Endocrinol 155:371-379.

Hukovic N, Panetta R, Kumar U, and Patel YC (1996) Agonist-dependent regulation of cloned human somatostatin receptor types 1-5 (hSSTR1-5): subtype selective internalization or upregulation. Endocrinology 137:4046-4049.

Hukovic N, Panetta R, Kumar U, Rocheville M, and Patel YC (1998) The cytoplasmic tail of the human somatostatin receptor type 5 is crucial for interaction with adenylyl cyclase and in mediating desensitization and internalization. $J$ Biol Chem 273:21416-21422.

Hukovic N, Rocheville M, Kumar U, Sasi R, Khare S, and Patel YC (1999) Agonistdependent up-regulation of human somatostatin receptor type 1 requires molecular signals in the cytoplasmic C-tail. J Biol Chem 274:24550-24558.
Hurth K, Enz A, Floersheim P, Gentsch C, Hoyer D, Langenegger D, Neumann P, Pfäffli P, Sorg D, Swoboda R, et al. (2007) SAR of the arylpiperazine moiety of obeline somatostatin sst1 receptor antagonists. Bioorg Med Chem Lett 17: 3988-3991.

Iacovazzo D, Carlsen E, Lugli F, Chiloiro S, Piacentini S, Bianchi A, Giampietro A, Mormando M, Clear AJ, Doglietto F, et al. (2016) Factors predicting pasireotide responsiveness in somatotroph pituitary adenomas resistant to first-generation somatostatin analogues: an immunohistochemical study. Eur J Endocrinol 174: $241-250$

Ibáñez-Costa A, López-Sánchez LM, Gahete MD, Rivero-Cortés E, Vázquez-Borrego MC, Gálvez MA de la Riva A, Venegas-Moreno E, Jiménez-Reina L, MorenoCarazo A, et al. (2017a) BIM-23A760 influences key functional endpoints in pituitary adenomas and normal pituitaries: molecular mechanisms underlying the differential response in adenomas. Sci Rep 7:42002

Ibáñez-Costa A, Luque RM, and Castaño JP (2017b) Cortistatin: a new link between the growth hormone/prolactin axis, stress, and metabolism. Growth Horm IGF Res 33:23-27.

Ibáñez-Costa A, Rivero-Cortés E, Vázquez-Borrego MC, Gahete MD, Jiménez-Reina L, Venegas-Moreno E, de la Riva A, Arráez MA, González-Molero I, Schmid HA, et al. (2016) Octreotide and pasireotide (dis)similarly inhibit pituitary tumor cells in vitro. $J$ Endocrinol 231:135-145.

Imhof AK, Glück L, Gajda M, Lupp A, Bräuer R, Schaible HG, and Schulz S (2011) Differential antiinflammatory and antinociceptive effects of the somatostatin analogs octreotide and pasireotide in a mouse model of immune-mediated arthritis. Arthritis Rheum 63:2352-2362.

Iwanaga T, Miki T, and Takahashi-Iwanaga H (2011) Restricted expression of somatostatin receptor 3 to primary cilia in the pancreatic islets and adenohypophysis of mice. Biomed Res 32:73-81.

Jacob JJ and Bevan JS (2014) Should all patients with acromegaly receive somatostatin analogue therapy before surgery and, if so, for how long? Clin Endocrinol (Oxf) 81:812-817.

Jacobs S and Schulz S (2008) Intracellular trafficking of somatostatin receptors. Mol Cell Endocrinol 286:58-62.

Jaffrain-Rea M-L, Rotondi S, Turchi A, Occhi G, Barlier A, Peverelli E, Rostomyan L, Defilles C, Angelini M, Oliva M-A, et al. (2013) Somatostatin analogues increase AIP expression in somatotropinomas, irrespective of Gsp mutations. Endocr Relat Cancer 20:753-766.

Jansson JO, Edén S, and Isaksson O (1985) Sexual dimorphism in the control of growth hormone secretion. Endocr Rev 6:128-150.

Jaquet P, Gunz G, Saveanu A, Dufour H, Taylor J, Dong J, Kim S, Moreau J-P, Enjalbert A, and Culler MD (2005) Efficacy of chimeric molecules directed towards multiple somatostatin and dopamine receptors on inhibition of $\mathrm{GH}$ and prolactin secretion from $\mathrm{GH}$-secreting pituitary adenomas classified as partially responsive to somatostatin analog therapy. Eur $J$ Endocrinol 153:135-141.

Jaquet P, Saveanu A, Gunz G, Fina F, Zamora AJ, Grino M, Culler MD, Moreau JP, Enjalbert A, and Ouafik LH (2000) Human somatostatin receptor subtypes in acromegaly: distinct patterns of messenger ribonucleic acid expression and hormone suppression identify different tumoral phenotypes. J Clin Endocrinol Metab 85 781-792.

Jiang N, Furue H, Katafuchi T, and Yoshimura M (2003) Somatostatin directly inhibits substantia gelatinosa neurons in adult rat spinal dorsal horn in vitro. Neurosci Res 47:97-107.

Jin H, White SR, Shida T, Schulz S, Aguiar M, Gygi SP, Bazan JF, and Nachury MV (2010) The conserved Bardet-Biedl syndrome proteins assemble a coat that traffics membrane proteins to cilia. Cell 141:1208-1219.

Johansson O, Hökfelt T, and Elde RP (1984) Immunohistochemical distribution of somatostatin-like immunoreactivity in the central nervous system of the adult rat. Neuroscience 13:265-339.

Johnson J, Wu V, Wong H, Walsh JH, and Brecha NC (1999) Somatostatin receptor subtype 2A expression in the rat retina. Neuroscience 94:675-683.

Jordan BA and Devi LA (1999) G-protein-coupled receptor heterodimerization modulates receptor function. Nature 399:697-700.

Kaemmerer D, Specht E, Sänger J, Wirtz RM, Sayeg M, Schulz S, and Lupp A (2015a) Somatostatin receptors in bronchopulmonary neuroendocrine neoplasms: new diagnostic, prognostic, and therapeutic markers. J Clin Endocrinol Metab 100: $831-840$

Kaemmerer D, Träger T, Hoffmeister M, Sipos B, Hommann M, Sänger J, Schulz S, and Lupp A (2015b) Inverse expression of somatostatin and CXCR4 chemokine receptors in gastroenteropancreatic neuroendocrine neoplasms of different malignancy. Oncotarget 6:27566-27579.

Kailey B, van de Bunt M, Cheley S, Johnson PR, MacDonald PE, Gloyn AL, Rorsman $\mathrm{P}$, and Braun M (2012) SSTR2 is the functionally dominant somatostatin receptor in human pancreatic $\beta$ - and $\alpha$-cells. Am $J$ Physiol Endocrinol Metab 303: E1107-E1116.

Kajiwara H, Hirabayashi K, Miyazawa M, Nakamura N, Hirasawa T, Muramatsu T, Mikami M, Yasuda M, and Osamura RY (2009) Immunohistochemical expression of somatostatin type $2 \mathrm{~A}$ receptor in neuroendocrine carcinoma of uterine cervix. Arch Gynecol Obstet 279:521-525.

Kaloudi A, Nock BA, Krenning EP, Maina T, and De Jong M (2015) Radiolabeled gastrin/CCK analogs in tumor diagnosis: towards higher stability and improved tumor targeting. Q J Nucl Med Mol Imaging 59:287-302.

Kaloudi A, Nock BA, Lymperis E, Valkema R, Krenning EP, de Jong M, and Maina T (2016) Impact of clinically tested NEP/ACE inhibitors on tumor uptake of [(111)InDOTA]MG11-first estimates for clinical translation. EJNMMI Res 6:15.

Kamenický P, Mazziotti G, Lombès M, Giustina A, and Chanson P (2014) Growth hormone, insulin-like growth factor-1, and the kidney: pathophysiological and clinical implications. Endocr Rev 35:234-281.

Karasawa H, Yakabi S, Wang L, Stengel A, Rivier J, and Taché Y (2014) Brain somatostatin receptor 2 mediates the dipsogenic effect of central somatostatin and cortistatin in rats: role in drinking behavior. Am J Physiol Regul Integr Comp Physiol 307:R793-R801. 
Kato M, Inoshita N, Sugiyama T, Tani Y, Shichiri M, Sano T, Yamada S, and Hirata Y (2012) Differential expression of genes related to drug responsiveness between sparsely and densely granulated somatotroph adenomas. Endocr $J$ 59:221-228.

Katznelson L, Laws ER Jr, Melmed S, Molitch ME, Murad MH, Utz A, and Wass JAH Endocrine Society (2014) Acromegaly: an endocrine society clinical practice guideline. J Clin Endocrinol Metab 99:3933-3951.

Khare S, Kumar U, Sasi R, Puebla L, Calderon L, Lemstrom K, Hayry P, and Patel AY (1999) Differential regulation of somatostatin receptor types 1-5 in rat aorta after angioplasty. FASEB $J$ 13:387-394.

Kiagiadaki F, Savvaki M, and Thermos K (2010) Activation of somatostatin receptor (sst 5) protects the rat retina from AMPA-induced neurotoxicity. Neuropharmacology 58:297-303.

Kim E, Sohn S, Lee M, Park C, Jung J, and Park S (2005) Effect of gsp oncogene on somatostatin receptor subtype 1 and 2 mRNA levels in GHRH-responsive GH3 cells. Pituitary 8:155-162.

Kim JK, Kwon O, Kim J, Kim EK, Park HK, Lee JE, Kim KL, Choi JW, Lim S, Seok $\mathrm{H}$, et al. (2012) PDZ domain-containing 1 (PDZK1) protein regulates phospholipase C- $\beta 3$ (PLC- $\beta 3$ )-specific activation of somatostatin by forming a ternary complex with PLC- $\beta 3$ and somatostatin receptors. J Biol Chem 287:21012-21024.

Kim S-J, Kim JW, Han S-W, Oh D-Y, Lee S-H, Kim D-W, Im S-A, Kim T-Y, Seog Heo D, and Bang Y-J (2010) Biological characteristics and treatment outcomes of metastatic or recurrent neuroendocrine tumors: tumor grade and metastatic site are important for treatment strategy. BMC Cancer 10:448.

Kim Y, Yang GR, Pradhan K, Venkataraju KU, Bota M, García Del Molino LC, Fitzgerald G, Ram K, He M, Levine JM, et al. (2017) Brain-wide maps reveal stereotyped cell-type-based cortical architecture and subcortical sexual dimorphism. Cell 171:456-469.e22.

Kimura N, Schindler M, Kasai N, and Kimura I (2001) Immunohistochemical localization of somatostatin receptor type $2 \mathrm{~A}$ in rat and human tissues. Endocr $J \mathbf{4 8}$ : 95-102.

Kiviniemi A, Gardberg M, Kivinen K, Posti JP, Vuorinen V, Sipilä J, Rahi M, Sankinen M, and Minn H (2017) Somatostatin receptor 2A in gliomas: association with oligodendrogliomas and favourable outcome. Oncotarget 8:49123-49132.

Kluxen FW, Bruns C, and Lübbert H (1992) Expression cloning of a rat brain somatostatin receptor cDNA. Proc Natl Acad Sci USA 89:4618-4622.

Komatsuzaki K, Terashita K, Kinane TB, and Nishimoto I (2001) Somatostatin type $\mathrm{V}$ receptor activates c-Jun N-terminal kinases via Galpha(12) family G proteins. Biochem Biophys Res Commun 289:1211-1217.

Kong G, Callahan J, Hofman MS, Pattison DA, Akhurst T, Michael M, Eu P, and Hicks RJ (2017) High clinical and morphologic response using ${ }^{90}$ Y-DOTAoctreotate sequenced with ${ }^{177} \mathrm{Lu}$-DOTA-octreotate induction peptide receptor chemoradionuclide therapy (PRCRT) for bulky neuroendocrine tumours. Eur J Nucl Med Mol Imaging 44:476-489.

Konturek SJ, Tasler J, Obtulowicz W, Coy DH, and Schally AV (1976) Effect of growth hormone-release inhibiting hormone on hormones stimulating exocrine pancreatic secretion. J Clin Invest 58:1-6.

Kosari F, Munz JM, Savci-Heijink CD, Spiro C, Klee EW, Kube DM, Tillmans L, Slezak J, Karnes RJ, Cheville JC, et al. (2008) Identification of prognostic biomarkers for prostate cancer. Clin Cancer Res 14:1734-1743.

Kozhemyakin M, Rajasekaran K, Todorovic MS, Kowalski SL, Balint C, and Kapur J (2013) Somatostatin type-2 receptor activation inhibits glutamate release and prevents status epilepticus. Neurobiol Dis 54:94-104.

Kreienkamp HJ (2008) Scaffolding proteins at the postsynaptic density: shank as the architectural framework. Handb Exp Pharmacol 186:365-380.

Kreienkamp HJ, Akgün E, Baumeister H, Meyerhof W, and Richter D (1999) Somatostatin receptor subtype 1 modulates basal inhibition of growth hormone release in somatotrophs. FEBS Lett 462:464-466.

Kreienkamp H-J, Hönck H-H, and Richter D (1997) Coupling of rat somatostatin receptor subtypes to a G-protein gated inwardly rectifying potassium channel (GIRK1). FEBS Lett 419:92-94.

Kreienkamp H-J, Larusson HJ, Witte I, Roeder T, Birgül N, Hönck H-H, Harder S, Ellinghausen G, Buck F, and Richter D (2002) Functional annotation of two orphan G-protein-coupled receptors, Drostar1 and -2, from Drosophila melanogaster and their ligands by reverse pharmacology. J Biol Chem 277:39937-39943.

Kreienkamp H-J, Roth A, and Richter D (1998) Rat somatostatin receptor subtype 4 can be made sensitive to agonist-induced internalization by mutation of a single threonine (residue 331). DNA Cell Biol 17:869-878.

Krenning EP, Bakker WH, Breeman WA, Koper JW, Kooij PP, Ausema L, Lameris JS, Reubi JC, and Lamberts SWJ (1989) Localisation of endocrine-related tumours with radioiodinated analogue of somatostatin. Lancet 1:242-244.

Krenning EP, Bakker WH, Kooij PPM, Breeman WAP, Oei HY, de Jong M, Reubi JC, Visser TJ, Bruns C, Kwekkeboom DJ, et al. (1992) Somatostatin receptor scintigraphy with indium-111-DTPA-D-Phe-1-octreotide in man: metabolism, dosimetry and comparison with iodine-123-Tyr-3-octreotide. J Nucl Med 33:652-658.

Krenning EP, Kwekkeboom DJ, Bakker WH, Breeman WAP, Kooij PPM, Oei HY, van Hagen M, Postema PTE, de Jong M, Reubi JC, et al. (1993) Somatostatin receptor scintigraphy with [111In-DTPA-D-Phe1]- and [123I-Tyr3]-octreotide: the Rotterdam experience with more than 1000 patients. Eur J Nucl Med 20:716-731.

Kreuzer OJ, Krisch B, Déry O, Bunnett NW, and Meyerhof W (2001) Agonistmediated endocytosis of rat somatostatin receptor subtype 3 involves $\beta$-arrestin and clathrin coated vesicles. $J$ Neuroendocrinol 13:279-287.

Kubota A, Yamada Y, Kagimoto S, Yasuda K, Someya Y, Ihara Y, Okamoto Y, Kozasa T, Seino S, and Seino Y (1994) Multiple effector coupling of somatostatin receptor subtype SSTR1. Biochem Biophys Res Commun 204:176-186.

Kulaksiz H, Eissele R, Rössler D, Schulz S, Höllt V, Cetin Y, and Arnold R (2002) Identification of somatostatin receptor subtypes $1,2 \mathrm{~A}, 3$, and 5 in neuroendocrine tumours with subtype specific antibodies. Gut 50:52-60.

Kumar U (2011) Cross-talk and modulation of signaling between somatostatin and growth factor receptors. Endocrine 40:168-180.

Kumar U, Grigorakis SI, Watt HL, Sasi R, Snell L, Watson P, and Chaudhari S (2005) Somatostatin receptors in primary human breast cancer: quantitative analysis of mRNA for subtypes 1--5 and correlation with receptor protein expression and tumor pathology. Breast Cancer Res Treat 92:175-186.

Kumar U, Laird D, Srikant CB, Escher E, and Patel YC (1997) Expression of the five somatostatin receptor (SSTR1-5) subtypes in rat pituitary somatotrophes: quantitative analysis by double-layer immunofluorescence confocal microscopy. Endocrinology 138:4473-4476.

Lahlou H, Fanjul M, Pradayrol L, Susini C, and Pyronnet S (2005) Restoration of functional gap junctions through internal ribosome entry site-dependent synthesis of endogenous connexins in density-inhibited cancer cells. Mol Cell Biol 25 $4034-4045$.

Lahlou H, Saint-Laurent N, Estève J-P, Eychène A, Pradayrol L, Pyronnet S, and Susini C (2003) sst2 Somatostatin receptor inhibits cell proliferation through Ras-, Rap1-, and B-Raf-dependent ERK2 activation. J Biol Chem 278: 39356-39371.

Lai Z, He S, Sherer EC, Wu Z, Yu Y, Ball R, Hong Q, Yang DX, Guo L, Li D, et al. (2015) Discovery of substituted (4-phenyl-1H-imidazol-2-yl)methanamine as potent somatostatin receptor 3 agonists. Bioorg Med Chem Lett 25:3520-3525.

Laklai H, Laval S, Dumartin L, Rochaix P, Hagedorn M, Bikfalvi A, Le Guellec S, Delisle MB, Schally AV, Susini C, et al. (2009) Thrombospondin-1 is a critical effector of oncosuppressive activity of sst2 somatostatin receptor on pancreatic cancer. Proc Natl Acad Sci USA 106:17769-17774.

Lambertini C, Barzaghi-Rinaudo P, D'Amato L, Schulz S, Nuciforo P, and Schmid HA (2013) Evaluation of somatostatin receptor subtype expression in human neuroendocrine tumors using two sets of new monoclonal antibodies. Regul Pept 187: $35-41$.

Lamberts SWJ (1988) The role of somatostatin in the regulation of anterior pituitary hormone secretion and the use of its analogs in the treatment of human pituitary tumors. Endocr Rev 9:417-436.

Lamberts SWJ, van der Lely A-J, de Herder WW, and Hofland LJ (1996) Octreotide. $N$ Engl $J$ Med 334:246-254.

Lancranjan I and Atkinson AB; Sandostatin LAR Group (1999) Results of a European multicentre study with Sandostatin LAR in acromegalic patients. Pituitary 1 $105-114$.

Landis CA, Harsh G, Lyons J, Davis RL, McCormick F, and Bourne HR (1990) Clinical characteristics of acromegalic patients whose pituitary tumors contain mutant Gs protein. J Clin Endocrinol Metab 71:1416-1420.

Lang A, Sakhnini E, Fidder HH, Maor Y, Bar-Meir S, and Chowers Y (2005) Somatostatin inhibits pro-inflammatory cytokine secretion from rat hepatic stellate cells. Liver Int 25:808-816.

Lania A, Mantovani G, and Spada A (2008) Genetic abnormalities of somatostatin receptors in pituitary tumors. Mol Cell Endocrinol 286:180-186.

Lanneau C, Bluet-Pajot MT, Zizzari P, Csaba Z, Dournaud P, Helboe L, Hoyer D, Pellegrini E, Tannenbaum GS, Epelbaum J, et al. (2000) Involvement of the Sst1 somatostatin receptor subtype in the intrahypothalamic neuronal network regulating growth hormone secretion: an in vitro and in vivo antisense study. Endocrinology 141:967-979.

Lapa C, Hänscheid H, Wild V, Pelzer T, Schirbel A, Werner RA, Droll S, Herrmann K, Buck AK, and Lückerath K (2016) Somatostatin receptor expression in small cell lung cancer as a prognostic marker and a target for peptide receptor radionuclide therapy. Oncotarget 7:20033-20040.

Larkin S, Reddy R, Karavitaki N, Cudlip S, Wass J, and Ansorge O (2013) Granulation pattern, but not GSP or GHR mutation, is associated with clinical characteristics in somatostatin-naive patients with somatotroph adenomas. Eur J Endocrinol 168:491-499.

Laval S, Laklai H, Fanjul M, Pucelle M, Laurell H, Billon-Galés A, Le Guellec S, Delisle MB, Sonnenberg A, Susini C, et al. (2014) Dual roles of hemidesmosomal proteins in the pancreatic epithelium: the phosphoinositide 3-kinase decides. Oncogene 33:1934-1944.

Law SF, Yasuda K, Bell GI, and Reisine T (1993) Gi alpha 3 and G(o) alpha selectively associate with the cloned somatostatin receptor subtype SSTR2. J Biol Chem 268:10721-10727.

Lee M, Lupp A, Mendoza N, Martin N, Beschorner R, Honegger J, Schlegel J, Shively T, Pulz E, Schulz S, et al. (2015) SSTR3 is a putative target for the medical treatment of gonadotroph adenomas of the pituitary. Endocr Relat Cancer 22: 111-119.

Lehmann A, Kliewer A, Günther T, Nagel F, and Schulz S (2016) Identification of phosphorylation sites regulating sst3 somatostatin receptor trafficking. Mol Endocrinol 30:645-659.

Lehmann A, Kliewer A, Märtens JC, Nagel F, and Schulz S (2014a) Carboxylterminal receptor domains control the differential dephosphorylation of somatostatin receptors by protein phosphatase 1 isoforms. PLoS One 9:e91526.

Lehmann A, Kliewer A, Schütz D, Nagel F, Stumm R, and Schulz S (2014b) Carboxylterminal multi-site phosphorylation regulates internalization and desensitization of the human sst2 somatostatin receptor. Mol Cell Endocrinol 387:44-51.

Lek M, Karczewski KJ, Minikel EV, Samocha KE, Banks E, Fennell T, O'DonnellLuria AH, Ware JS, Hill AJ, Cummings BB, et al.; Exome Aggregation Consortium (2016) Analysis of protein-coding genetic variation in 60,706 humans. Nature 536: $285-291$.

Lelouvier B, Tamagno G, Kaindl AM, Roland A, Lelievre V, Le Verche V, Loudes C, Gressens P, Faivre-Baumann A, Lenkei Z, et al. (2008) Dynamics of somatostatin type 2A receptor cargoes in living hippocampal neurons. J Neurosci 28:4336-4349. Leontiou CA, Gueorguiev M, van der Spuy J, Quinton R, Lolli F, Hassan S, Chahal HS, Igreja SC, Jordan S, Rowe J, et al. (2008) The role of the aryl hydrocarbon receptor-interacting protein gene in familial and sporadic pituitary adenomas. $J$ Clin Endocrinol Metab 93:2390-2401.

Lepousez G, Mouret A, Loudes C, Epelbaum J, and Viollet C (2010) Somatostatin contributes to in vivo gamma oscillation modulation and odor discrimination in the olfactory bulb. J Neurosci 30:870-875.

Lesche S, Lehmann D, Nagel F, Schmid HA, and Schulz S (2009) Differential effects of octreotide and pasireotide on somatostatin receptor internalization and trafficking in vitro. J Clin Endocrinol Metab 94:654-661. 
Levy MJ, Bejon P, Barakat M, Goadsby PJ, and Meeran K (2003) Acromegaly: a unique human headache model. Headache 43:794-797.

Li D, Tanaka M, Brunicardi FC, Fisher WE, Gibbs RA, and Gingras MC (2011) Association between somatostatin receptor 5 gene polymorphisms and pancreatic cancer risk and survival. Cancer 117:2863-2872.

Li D, Wu Z, Yu Y, Ball RG, Guo L, Sherer E, He S, Hong Q, Lai Z, Qi H, et al. (2014) Diamine derivatives as novel small-molecule, potent, and subtype-selective somatostatin SST3 receptor agonists. ACS Med Chem Lett 5:690-695.

Li JK, Chow CC, Yeung VT, Mak TW, Ko GT, Swaminathan R, Chan JC, and Cockram CS (2000) Treatment of Chinese acromegaly with a combination of bromocriptine and octreotide. Aust N Z J Med 30:457-461.

Li XJ, Forte M, North RA, Ross CA, and Snyder SH (1992) Cloning and expression of a rat somatostatin receptor enriched in brain. J Biol Chem 267:21307-21312.

Liapakis G, Hoeger C, Rivier J, and Reisine T (1996) Development of a selective agonist at the somatostatin receptor subtype sstr1. J Pharmacol Exp Ther 276: 1089-1094.

Libert F, Parmentier M, Lefort A, Dinsart C, Van Sande J, Maenhaut C, Simons MJ, Dumont JE, and Vassart G (1989) Selective amplification and cloning of four new members of the G protein-coupled receptor family. Science 244:569-572.

Liebow C, Reilly C, Serrano M, and Schally AV (1989) Somatostatin analogues inhibit growth of pancreatic cancer by stimulating tyrosine phosphatase. Proc Natl Acad Sci USA 86:2003-2007.

Liew CW, Vockel M, Glassmeier G, Brandner JM, Fernandez-Ballester GJ, Schwarz JR, Schulz S, Buck F, Serrano L, Richter D, et al. (2009) Interaction of the human somatostatin receptor 3 with the multiple PDZ domain protein MUPP1 enables somatostatin to control permeability of epithelial tight junctions. FEBS Lett 583:49-54.

Lin C-Y, Varma MG, Joubel A, Madabushi S, Lichtarge O, and Barber DL (2003) Conserved motifs in somatostatin, D2-dopamine, and $\alpha 2 \mathrm{~B}$-adrenergic receptors for inhibiting the Na-H exchanger, NHE1. J Biol Chem 278:15128-15135.

Lin X, Voyno-Yasenetskaya TA, Hooley R, Lin CY, Orlowski J, and Barber DL (1996) Galpha12 differentially regulates $\mathrm{Na}+\mathrm{H}+$ exchanger isoforms. $J$ Biol Chem $\mathbf{2 7 1}$ 22604-22610.

Liu Q, Bee MS, and Schonbrunn A (2009) Site specificity of agonist and second messenger-activated kinases for somatostatin receptor subtype 2A (Sst2A) phosphorylation. Mol Pharmacol 76:68-80.

Liu Q, Cescato R, Dewi DA, Rivier J, Reubi JC, and Schonbrunn A (2005) Receptor signaling and endocytosis are differentially regulated by somatostatin analogs. $\mathrm{Mol}$ Pharmacol 68:90-101.

Liu Q, Dewi DA, Liu W, Bee MS, and Schonbrunn A (2008) Distinct phosphorylation sites in the SST2A somatostatin receptor control internalization, desensitization, and arrestin binding. Mol Pharmacol 73:292-304.

Liu Q, Reubi JC, Wang Y, Knoll BJ, and Schonbrunn A (2003) In vivo phosphorylation of the somatostatin 2A receptor in human tumors. J Clin Endocrinol Metab 88:6073-6079

Liu Q and Schonbrunn A (2001) Agonist-induced phosphorylation of somatostatin receptor subtype 1 (sst1): relationship to desensitization and internalization. J Biol Chem 276:3709-3717.

Liu S, Tang C, Ho B, Ankersen M, Stidsen CE, and Crider AM (1998) Nonpeptide somatostatin agonists with sst4 selectivity: synthesis and structure-activity relationships of thioureas. J Med Chem 41:4693-4705.

Liu Y, Lu D, Zhang Y, Li S, Liu X, and Lin H (2010) The evolution of somatostatin in vertebrates. Gene 463:21-28.

Liu Z, Crider AM, Ansbro D, Hayes C, and Kontoyianni M (2012) A structure-based approach to understanding somatostatin receptor-4 agonism (sst4). J Chem Inf Model 52:171-186.

Lopez F, Estève J-P, Buscail L, Delesque N, Saint-Laurent N, Théveniau M, Nahmias C, Vaysse N, and Susini C (1997) The tyrosine phosphatase SHP-1 associates with the sst2 somatostatin receptor and is an essential component of sst2-mediated inhibitory growth signaling. J Biol Chem 272:24448-24454.

Lopez F, Ferjoux G, Cordelier P, Saint-Laurent N, Estève JP, Vaysse N, Buscail L, and Susini C (2001) Neuronal nitric oxide synthase: a substrate for SHP-1 involved in sst2 somatostatin receptor growth inhibitory signaling. FASEB J 15:2300-2302.

Low MJ, Otero-Corchon V, Parlow AF, Ramirez JL, Kumar U, Patel YC, and Rubinstein M (2001) Somatostatin is required for masculinization of growth hormone-regulated hepatic gene expression but not of somatic growth. J Clin Invest 107:1571-1580.

Lublin AL, Diehl NL, and Hochgeschwender U (1997) Isolation and characterization of the gene encoding the type 5 mouse (Mus musculus) somatostatin receptor (msst5). Gene 195:63-66.

Ludvigsen E, Carlsson C, Tiensuu Janson E, Sandler S, and Stridsberg M (2015) Somatostatin receptor 1-5; expression profiles during rat development. Ups J Med Sci 120:157-168.

Lupp A, Hunder A, Petrich A, Nagel F, Doll C, and Schulz S (2011) Reassessment of sst(5) somatostatin receptor expression in normal and neoplastic human tissues using the novel rabbit monoclonal antibody UMB-4. Neuroendocrinology 94:255-264.

Lupp A, Nagel F, Doll C, Röcken C, Evert M, Mawrin C, Saeger W, and Schulz S (2012) Reassessment of sst3 somatostatin receptor expression in human normal and neoplastic tissues using the novel rabbit monoclonal antibody UMB-5. Neuroendocrinology 96:301-310.

Lupp A, Nagel F, and Schulz S (2013) Reevaluation of sst $_{1}$ somatostatin receptor expression in human normal and neoplastic tissues using the novel rabbit monoclonal antibody UMB-7. Regul Pept 183:1-6.

Luque RM, Durán-Prado M, García-Navarro S, Gracia-Navarro F, Kineman RD, Malagón MM, and Castaño JP (2006) Identification of the somatostatin receptor subtypes (sst) mediating the divergent, stimulatory/inhibitory actions of somatostatin on growth hormone secretion. Endocrinology 147:2902-2908

Luque RM, Ibáñez-Costa A, Neto LV, Taboada GF, Hormaechea-Agulla D, Kasuki L, Venegas-Moreno E, Moreno-Carazo A, Gálvez MA, Soto-Moreno A, et al. (2015) Truncated somatostatin receptor variant sst5TMD4 confers aggressive features (proliferation, invasion and reduced octreotide response) to somatotropinomas. Cancer Lett 359:299-306.
Luque RM, Park S, Peng XD, Delgado E, Gracia-Navarro F, Kineman RD, Malagón MM, and Castaño JP (2004) Homologous and heterologous in vitro regulation of pig pituitary somatostatin receptor subtypes, sst1, sst2 and sst5 mRNA. $J \mathrm{Mol}$ Endocrinol 32:437-448.

Maina T, Cescato R, Waser B, Tatsi A, Kaloudi A, Krenning EP, de Jong M, Nock BA and Reubi JC (2014) [111In-DOTA]LTT-SS28, a first pansomatostatin radioligand for in vivo targeting of somatostatin receptor-positive tumors. $J$ Med Chem $\mathbf{5 7}$ 6564-6571.

Maiza JC, Vezzosi D, Matta M, Donadille F, Loubes-Lacroix F, Cournot M, Bennet A, and Caron P (2007) Long-term (up to 18 years) effects on GH/IGF-1 hypersecretion and tumour size of primary somatostatin analogue (SSTa) therapy in patients with GH-secreting pituitary adenoma responsive to SSTa. Clin Endocrinol (Oxf) 67:282-289. Malagon MM, Molina M, Gahete MD, Durán-Prado M, Martinez-Fuentes AJ, Alcain FJ, Tonon MC, Leprince J, Vaudry H, Castaño JP, et al. (2008) Urotensin II and urotensin II-related peptide activate somatostatin receptor subtypes 2 and 5 . Peptides 29:711-720.

Malcangio M (2003) GDNF and somatostatin in sensory neurones. Curr Opin Pharmacol 3:41-45.

Mandarino L, Stenner D, Blanchard W, Nissen S, Gerich J, Ling N, Brazeau P, Bohlen P, Esch F, and Guillemin R (1981) Selective effects of somatostatin-14, -25 and -28 on in vitro insulin and glucagon secretion. Nature 291:76-77.

Marazioti A, Pitychoutis PM, Papadopoulou-Daifoti Z, Spyraki C, and Thermos K (2008) Activation of somatostatin receptors in the globus pallidus increases rat locomotor activity and dopamine release in the striatum. Psychopharmacology (Berl) 201:413-422.

Marina D, Burman P, Klose M, Casar-Borota O, Luque RM, Castaño JP, and FeldtRasmussen U (2015) Truncated somatostatin receptor 5 may modulate therapy response to somatostatin analogues-observations in two patients with acromegaly and severe headache. Growth Horm IGF Res 25:262-267.

Martin RE, Mohr P, Maerki HP, Guba W, Kuratli C, Gavelle O, Binggeli A, Bendels S, Alvarez-Sánchez R, Alker A, et al. (2009) Benzoxazole piperidines as selective and potent somatostatin receptor subtype 5 antagonists. Bioorg Med Chem Lett 19:6106-6113.

Martínez V, Rivier J, Coy D, and Taché Y (2000) Intracisternal injection of somatostatin receptor 5-preferring agonists induces a vagal cholinergic stimulation of gastric emptying in rats. J Pharmacol Exp Ther 293:1099-1105.

Marzullo P, Ferone D, Di Somma C, Pivonello R, Filippella M, Lombardi G, and Colao A (1999) Efficacy of combined treatment with lanreotide and cabergoline in selected therapy-resistant acromegalic patients. Pituitary 1:115-120.

Massa A, Barbieri F, Aiello C, Arena S, Pattarozzi A, Pirani P, Corsaro A, Iuliano R, Fusco A, Zona G, et al. (2004) The expression of the phosphotyrosine phosphatase DEP-1/PTPeta dictates the responsivity of glioma cells to somatostatin inhibition of cell proliferation. J Biol Chem 279:29004-29012.

Mastrodimou N and Thermos K (2004) The somatostatin receptor (sst1) modulates the release of somatostatin in rat retina. Neurosci Lett 356:13-16.

Matei DV, Renne G, Pimentel M, Sandri MT, Zorzino L, Botteri E, De Cicco C, Musi G, Brescia A, Mazzoleni F, et al. (2012) Neuroendocrine differentiation in castration-resistant prostate cancer: a systematic diagnostic attempt. Clin Genitourin Cancer 10:164-173.

Matrone C, Pivonello R, Colao A, Cappabianca P, Cavallo LM, Del Basso De Caro ML, Taylor JE, Culler MD, Lombardi G, Di Renzo GF, et al. (2004) Expression and function of somatostatin receptor subtype 1 in human growth hormone secreting pituitary tumors deriving from patients partially responsive or resistant to longterm treatment with somatostatin analogs. Neuroendocrinology 79:142-148.

Mawrin C, Schulz S, Hellwig-Patyk A, Kirches E, Roessner A, Lendeckel U, Firsching R, Vorwerk CK, Keilhoff G, Dietzmann K, et al. (2005) Expression and function of somatostatin receptors in peripheral nerve sheath tumors. $J$ Neuropathol Exp Neurol 64:1080-1088.

Mawrin C, Schulz S, Pauli SU, Treuheit T, Diete S, Dietzmann K, Firsching R, Schulz S, and Höllt V (2004) Differential expression of sst1, sst2A, and sst3 somatostatin receptor proteins in low-grade and high-grade astrocytomas. $J$ Neuropathol Exp Neurol 63:13-19.

Mayr B, Buslei R, Theodoropoulou M, Stalla GK, Buchfelder M, and Schöfl C (2013) Molecular and functional properties of densely and sparsely granulated GH-producing pituitary adenomas. Eur J Endocrinol 169:391-400.

Mazziotti G, Floriani I, Bonadonna S, Torri V, Chanson P, and Giustina A (2009) Effects of somatostatin analogs on glucose homeostasis: a metaanalysis of acromegaly studies. J Clin Endocrinol Metab 94:1500-1508.

McCosh RB, Szeligo BM, Bedenbaugh MN, Lopez JA, Hardy SL, Hileman SM, Lehman MN, and Goodman RL (2017) Evidence that endogenous somatostatin inhibits episodic, but not surge, secretion of LH in female sheep. Endocrinology 158:1827-1837.

Medina DL, Velasco JA, and Santisteban P (1999) Somatostatin is expressed in FRTL-5 thyroid cells and prevents thyrotropin-mediated down-regulation of the cyclin-dependent kinase inhibitor p27kip1. Endocrinology 140:87-95.

Mehta S, de Reuver PR, Gill P, Andrici J, D’Urso L, Mittal A, Pavlakis N, Clarke S, Samra JS, and Gill AJ (2015) Somatostatin receptor SSTR-2a expression is a stronger predictor for survival than Ki-67 in pancreatic neuroendocrine tumors. Medicine (Baltimore) 94:e1281.

Meisetschläger G, Poethko T, Stahl A, Wolf I, Scheidhauer K, Schottelius M, Herz M, Wester HJ, and Schwaiger M (2006) Gluc-Lys([18F]FP)-TOCA PET in patients with SSTR-positive tumors: biodistribution and diagnostic evaluation compared with [111In]DTPA-octreotide. J Nucl Med 47:566-573.

Melmed S (2003) Mechanisms for pituitary tumorigenesis: the plastic pituitary. $J$ Clin Invest 112:1603-1618.

Melmed S (2016) Pituitary medicine from discovery to patient-focused outcomes. $J$ Clin Endocrinol Metab 101:769-777.

Melmed S, Braunstein GD, Horvath E, Ezrin C, and Kovacs K (1983) Pathophysiology of acromegaly. Endocr Rev 4:271-290.

Melzer S, Michael M, Caputi A, Eliava M, Fuchs EC, Whittington MA, and Monyer H (2012) Long-range-projecting GABAergic neurons modulate inhibition in hippocampus and entorhinal cortex. Science 335:1506-1510. 
Mergler S, Singh V, Grötzinger C, Kaczmarek P, Wiedenmann B, and Strowski MZ (2008) Characterization of voltage operated R-type Ca2+ channels in modulating somatostatin receptor subtype 2- and 3-dependent inhibition of insulin secretion from INS-1 cells. Cell Signal 20:2286-2295.

Meyerhof W, Paust HJ, Schönrock C, and Richter D (1991) Cloning of a cDNA encoding a novel putative G-protein-coupled receptor expressed in specific rat brain regions. DNA Cell Biol 10:689-694.

Meyerhof W, Wulfsen I, Schönrock C, Fehr S, and Richter D (1992) Molecular cloning of a somatostatin-28 receptor and comparison of its expression pattern with that of a somatostatin-14 receptor in rat brain. Proc Natl Acad Sci USA 89:10267-10271.

Meyers C, Arimura A, Gordin A, Fernandez-Durango R, Coy DH, Schally AV, Drouin J, Ferland L, Beaulieu M, and Labrie F (1977) Somatostatin analogs which inhibit glucagon and growth hormone more than insulin release. Biochem Biophys Res Commun 74:630-636.

Mezey E, Hunyady B, Mitra S, Hayes E, Liu Q, Schaeffer J, and Schonbrunn A (1998) Cell specific expression of the sst2A and sst5 somatostatin receptors in the rat anterior pituitary. Endocrinology 139:414-419.

Minniti G, Jaffrain-Rea ML, Baldelli R, Ferretti E, Caracciolo B, Bultrini A, Gulino A, and Tamburrano G (1997) Acute effects of octreotide, cabergoline and a combination of both drugs on GH secretion in acromegalic patients. Clin Ter 148: 601-607.

Misawa K, Misawa Y, Kondo H, Mochizuki D, Imai A, Fukushima H, Uehara T, Kanazawa T, and Mineta H (2015) Aberrant methylation inactivates somatostatin and somatostatin receptor type 1 in head and neck squamous cell carcinoma. PLoS One 10: 0118588.

Mizutani G, Nakanishi Y, Watanabe N, Honma T, Obana Y, Seki T, Ohni S, and Nemoto N (2012) Expression of somatostatin receptor (SSTR) subtypes (SSTR$1,2 \mathrm{~A}, 3,4$ and 5) in neuroendocrine tumors using real-time RT-PCR method and immunohistochemistry. Acta Histochem Cytochem 45:167-176.

Moatassim-Billah S, Duluc C, Samain R, Jean C, Perraud A, Decaup E, CassantSourdy S, Bakri Y, Selves J, Schmid H, et al. (2016) Anti-metastatic potential of somatostatin analog SOM230: indirect pharmacological targeting of pancreatic cancer-associated fibroblasts. Oncotarget 7:41584-41598.

Modlin IM, Latich I, Kidd M, Zikusoka M, and Eick G (2006) Therapeutic options for gastrointestinal carcinoids. Clin Gastroenterol Hepatol 4:526-547.

Moldovan S, DeMayo F, and Brunicardi FC (1998) Cloning of the mouse SSTR5 gene. $J$ Surg Res 76:57-60.

Molè D, Gentilin E, Ibañez-Costa A, Gagliano T, Gahete MD, Tagliati F, Rossi R, Pelizzo MR, Pansini G, Luque RM, et al. (2015) The expression of the truncated isoform of somatostatin receptor subtype 5 associates with aggressiveness in medullary thyroid carcinoma cells. Endocrine 50:442-452.

Møller LN, Stidsen CE, Hartmann B, and Holst JJ (2003) Somatostatin receptors. Biochim Biophys Acta 1616:1-84.

Momiyama T and Zaborszky L (2006) Somatostatin presynaptically inhibits both GABA and glutamate release onto rat basal forebrain cholinergic neurons. $J$ Neurophysiol 96:686-694.

Moneta D, Richichi C, Aliprandi M, Dournaud P, Dutar P, Billard JM, Carlo AS, Viollet C, Hannon JP, Fehlmann D, et al. (2002) Somatostatin receptor subtypes 2 and 4 affect seizure susceptibility and hippocampal excitatory neurotransmission in mice. Eur J Neurosci 16:843-849.

Montminy M, Brindle P, Arias J, Ferreri K, and Armstrong R (1996) Regulation of somatostatin gene transcription by cyclic adenosine monophosphate. Metabolism 45 (Suppl 1):4-7.

Montminy MR, Goodman RH, Horovitch SJ, and Habener JF (1984) Primary structure of the gene encoding rat preprosomatostatin. Proc Natl Acad Sci USA 81 $3337-3340$

Moore SD, Madamba SG, Joëls M, and Siggins GR (1988) Somatostatin augments the M-current in hippocampal neurons. Science 239:278-280.

Morell M, Souza-Moreira L, Caro M, O’Valle F, Forte-Lago I, de Lecea L, GonzalezRey E, and Delgado M (2013) Analgesic effect of the neuropeptide cortistatin in murine models of arthritic inflammatory pain. Arthritis Rheum 65:1390-1401.

Mulak A, Larauche M, Biraud M, Million M, Rivier J, and Taché Y (2015) Selective agonists of somatostatin receptor subtype 1 or 2 injected peripherally induce antihyperalgesic effect in two models of visceral hypersensitivity in mice. Peptides 63:71-80

Müller EE, Locatelli V, and Cocchi D (1999) Neuroendocrine control of growth hormone secretion. Physiol Rev 79:511-607.

Mullershausen F, Zecri F, Cetin C, Billich A, Guerini D, and Seuwen K (2009) Persistent signaling induced by FTY720-phosphate is mediated by internalized S1P1 receptors. Nat Chem Biol 5:428-434.

Murray RD and Melmed S (2008) A critical analysis of clinically available somatostatin analog formulations for therapy of acromegaly. J Clin Endocrinol Metab 93:2957-2968.

Musolino NR, Marino Júnior R, and Bronstein MD (1990) Headache in acromegaly: dramatic improvement with the somatostatin analogue SMS 201-995. Clin J Pain 6:243-245

Müssig K, Wehrmann T, Dittmann H, Wehrmann M, Ueberberg B, Schulz S, Bares $\mathrm{R}$, and Petersenn S (2012) Expression of the proliferation marker Ki-67 associates with tumour staging and clinical outcome in differentiated thyroid carcinomas. Clin Endocrinol (Oxf) 77:139-145.

Nagel F, Doll C, Pöll F, Kliewer A, Schröder H, and Schulz S (2011) Structural determinants of agonist-selective signaling at the $\mathrm{sst}(2 \mathrm{~A})$ somatostatin receptor. Mol Endocrinol 25:859-866.

Nager AR, Goldstein JS, Herranz-Pérez V, Portran D, Ye F, Garcia-Verdugo JM, and Nachury MV (2017) An actin network dispatches ciliary GPCRs into extracellular vesicles to modulate signaling. Cell 168:252-263.e14.

Najib S, Saint-Laurent N, Estève JP, Schulz S, Boutet-Robinet E, Fourmy D, Lättig J, Mollereau C, Pyronnet S, Susini C, et al. (2012) A switch of G protein-coupled receptor binding preference from phosphoinositide 3-kinase (PI3K)-p85 to filamin A negatively controls the PI3K pathway. Mol Cell Biol 32:1004-1016.

Neggers SJ, Pronin V, Balcere I, Lee M-K, Rozhinskaya L, Bronstein MD, Gadelha MR, Maisonobe P, Sert C, and van der Lely AJ; LEAD Study Group (2015) Lanreotide Autogel $120 \mathrm{mg}$ at extended dosing intervals in patients with acromegaly biochemically controlled with octreotide LAR: the LEAD study. Eur $J$ Endocrinol 173:313-323.

Nehring RB, Meyerhof W, and Richter D (1995) Aspartic acid residue 124 in the third transmembrane domain of the somatostatin receptor subtype 3 is essential for somatostatin-14 binding. DNA Cell Biol 14:939-944.

Nicolas GP, Mansi R, McDougall L, Kaufmann J, Bouterfa H, Wild D, and Fani M (2017) Biodistribution, pharmacokinetics, and dosimetry of ${ }^{177} \mathrm{Lu}-,{ }^{90} \mathrm{Y}-$, and ${ }^{111}$ In-labeled somatostatin receptor antagonist OPS201 in comparison to the agonist ${ }^{177}$ Lu-DOTATATE: the mass effect. J Nucl Med 58:1435-1441.

Nicolas GP, Schreiter N, Kaul F, Uiters J, Bouterfa H, Kaufmann J, Erlanger TE, Cathomas R, Christ E, Fani M, et al. (2018) Comparison of (68)Ga-OPS202 (68)GaNODAGA-JR11) and (68)Ga-DOTATOC ((68)Ga-edotreotide) PET/CT in patients with gastroenteropancreatic neuroendocrine tumors: evaluation of sensitivity in a prospective phase II imaging study. J Nucl Med 59:909-914.

Nilsson A, Stroth N, Zhang X, Qi H, Fälth M, Sköld K, Hoyer D, Andrén PE, and Svenningsson P (2012) Neuropeptidomics of mouse hypothalamus after imipramine treatment reveal somatostatin as a potential mediator of antidepressant effects. Neuropharmacology 62:347-357.

Noam Y, Ehrengruber MU, Koh A, Feyen P, Manders EM, Abbott GW, Wadman WJ, and Baram TZ (2014) Filamin A promotes dynamin-dependent internalization of hyperpolarization-activated cyclic nucleotide-gated type 1 (HCN1) channels and restricts Ih in hippocampal neurons. J Biol Chem 289:5889-5903.

Nock BA, Maina T, Krenning EP, and de Jong M (2014) "To serve and protect": enzyme inhibitors as radiopeptide escorts promote tumor targeting. $J$ Nucl Med 55:121-127.

Nouel D, Gaudriault G, Houle M, Reisine T, Vincent JP, Mazella J, and Beaudet A (1997) Differential internalization of somatostatin in COS-7 cells transfected with SST1 and SST2 receptor subtypes: a confocal microscopic study using novel fluorescent somatostatin derivatives. Endocrinology 138:296-306.

Nunes VS, Correa JMS, Puga MES, Silva EMK, and Boguszewski CL (2015) Preoperative somatostatin analogues versus direct transsphenoidal surgery for newlydiagnosed acromegaly patients: a systematic review and meta-analysis using the GRADE system. Pituitary 18:500-508.

Nyegaard M, Børglum AD, Bruun TG, Collier DA, Russ C, Mors O, Ewald H, and Kruse TA (2002) Novel polymorphisms in the somatostatin receptor 5 (SSTR5) gene associated with bipolar affective disorder. Mol Psychiatry 7:745-754.

Oakley RH, Laporte SA, Holt JA, Caron MG, and Barak LS (2000) Differential affinities of visual arrestin, beta arrestin1, and beta arrestin2 for G protein-coupled receptors delineate two major classes of receptors. J Biol Chem 275:17201-17210.

Öberg K and Lamberts SWJ (2016) Somatostatin analogues in acromegaly and gastroenteropancreatic neuroendocrine tumours: past, present and future. Endocr Relat Cancer 23:R551-R566.

O'Carroll AM, Lolait SJ, König M, and Mahan LC (1992) Molecular cloning and expression of a pituitary somatostatin receptor with preferential affinity for somatostatin-28. Mol Pharmacol 42:939-946.

Ocampo Daza D, Sundström G, Bergqvist CA, and Larhammar D (2012) The evolution of vertebrate somatostatin receptors and their gene regions involves extensive chromosomal rearrangements. BMC Evol Biol 12:231.

Okazaki T, Maeda A, Nishimura H, Kurosaki T, and Honjo T (2001) PD-1 immunoreceptor inhibits B cell receptor-mediated signaling by recruiting src homology 2-domain-containing tyrosine phosphatase 2 to phosphotyrosine. Proc Natl Acad Sci USA 98:13866-13871.

Okuwaki K, Kida M, Mikami T, Yamauchi H, Imaizumi H, Miyazawa S, Iwai T, Takezawa M, Saegusa M, Watanabe M, et al. (2013) Clinicopathologic characteristics of pancreatic neuroendocrine tumors and relation of somatostatin receptor type 2A to outcomes. Cancer 119:4094-4102.

Olias G, Viollet C, Kusserow H, Epelbaum J, and Meyerhof W (2004) Regulation and function of somatostatin receptors. $J$ Neurochem 89:1057-1091

Onoprishvili I, Andria ML, Kramer HK, Ancevska-Taneva N, Hiller JM, and Simon EJ (2003) Interaction between the mu opioid receptor and filamin A is involved in receptor regulation and trafficking. Mol Pharmacol 64:1092-1100.

Oriola J, Lucas T, Halperin I, Mora M, Perales MJ, Alvarez-Escolá C, Paz MN, Díaz Soto G, Salinas I, Julián MT, et al. (2012) Germline mutations of AIP gene in somatotropinomas resistant to somatostatin analogues. Eur J Endocrinol 168: 9-13.

Orlando C, Raggi CC, Bianchi S, Distante V, Simi L, Vezzosi V, Gelmini S, Pinzani P, Smith MC, Buonamano A, et al. (2004) Measurement of somatostatin receptor subtype 2 mRNA in breast cancer and corresponding normal tissue. Endocr Relat Cancer 11:323-332.

Osterstock G, Mitutsova V, Barre A, Granier M, Fontanaud P, Chazalon M, Carmignac D, Robinson IC, Low MJ, Plesnila N, et al. (2016) Somatostatin triggers rhythmic electrical firing in hypothalamic GHRH neurons. Sci Rep 6:24394.

Otte A, Jermann E, Behe M, Goetze M, Bucher HC, Roser HW, Heppeler A, MuellerBrand J, and Maecke HR (1997) DOTATOC: a powerful new tool for receptormediated radionuclide therapy. Eur J Nucl Med 24:792-795.

Paganelli G, Zoboli S, Cremonesi M, Mäcke HR, and Chinol M (1999) Receptormediated radionuclide therapy with 90Y-DOTA-D-Phe1-Tyr3-octreotide: preliminary report in cancer patients. Cancer Biother Radiopharm 14:477-483.

Pagès P, Benali N, Saint-Laurent N, Estève J-P, Schally AV, Tkaczuk J, Vaysse N, Susini C, and Buscail L (1999) sst2 somatostatin receptor mediates cell cycle arrest and induction of p27(Kip1): evidence for the role of SHP-1. J Biol Chem 274: 15186-15193.

Palii SS, Afzal A, Shaw LC, Pan H, Caballero S, Miller RC, Jurczyk S, Reubi JC, Tan Y, Hochhaus G, et al. (2008) Nonpeptide somatostatin receptor agonists specifically target ocular neovascularization via the somatostatin type 2 receptor. Invest Ophthalmol Vis Sci 49:5094-5102.

Palmieri G, Montella L, Aiello C, Barbieri F, Di Vizio D, Schulz S, Beninati S, Budillon A Caraglia M, Insabato L, et al. (2007) Somatostatin analogues, a series of tissue transglutaminase inducers, as a new tool for therapy of mesenchimal tumors of the gastrointestinal tract. Amino Acids 32:395-400.

Pan MG, Florio T, and Stork PJ (1992) G protein activation of a hormone-stimulated phosphatase in human tumor cells. Science 256:1215-1217. 
Panetta R, Greenwood MT, Warszynska A, Demchyshyn LL, Day R, Niznik HB, Srikant CB, and Patel YC (1994) Molecular cloning, functional characterization, and chromosomal localization of a human somatostatin receptor (somatostatin receptor type 5) with preferential affinity for somatostatin-28. Mol Pharmacol 45: 417-427.

Panetta R and Patel YC (1995) Expression of mRNA for all five human somatostatin receptors (hSSTR1-5) in pituitary tumors. Life Sci 56:333-342.

Papotti M, Kumar U, Volante M, Pecchioni C, and Patel YC (2001) Immunohistochemical detection of somatostatin receptor types 1-5 in medullary carcinoma of the thyroid. Clin Endocrinol (Oxf) 54:641-649.

Park S, Jiang H, Zhang H, and Smith RG (2012) Modification of ghrelin receptor signaling by somatostatin receptor-5 regulates insulin release. Proc Natl Acad Sci USA 109:19003-19008.

Park S, Kamegai J, Johnson TA, Frohman LA, and Kineman RD (2000) Modulation of pituitary somatostatin receptor subtype (sst1-5) messenger ribonucleic acid levels by changes in the growth hormone axis. Endocrinology 141:3556-3563.

Pasternak A, Feng Z, de Jesus R, Ye Z, He S, Dobbelaar P, Bradley SA, Chicchi GG, Tsao K-L, Trusca D, et al. (2012) Stimulation of glucose-dependent insulin secretion by a potent, selective sst3 antagonist. ACS Med Chem Lett 3:289-293.

Patel YC (1999) Somatostatin and its receptor family. Front Neuroendocrinol 20 157-198.

Patel YC, Greenwood M, Panetta R, Hukovic N, Grigorakis S, Robertson LA, and Srikant CB (1996) Molecular biology of somatostatin receptor subtypes. $\mathrm{Me}$ tabolism 45 (Suppl 1):31-38.

Patel YC, Greenwood MT, Warszynska A, Panetta R, and Srikant CB (1994) All five cloned human somatostatin receptors (hSSTR1-5) are functionally coupled to adenylyl cyclase. Biochem Biophys Res Commun 198:605-612.

Pavan B, Fiorini S, Dal Monte M, Lunghi L, Biondi C, Bagnoli P, and Cervia D (2004) Somatostatin coupling to adenylyl cyclase activity in the mouse retina. Naunyn Schmiedebergs Arch Pharmacol 370:91-98.

Pavel ME, Hainsworth JD, Baudin E, Peeters M, Hörsch D, Winkler RE, Klimovsky J, Lebwohl D, Jehl V, Wolin EM, et al.; RADIANT-2 Study Group (2011) Everolimus plus octreotide long-acting repeatable for the treatment of advanced neuroendocrine tumours associated with carcinoid syndrome (RADIANT-2): a randomised, placebo-controlled, phase 3 study. Lancet 378:2005-2012

Pazaitou-Panayiotou K, Tiensuu Janson E, Koletsa T, Kotoula V, Stridsberg M, Karkavelas G, and Karayannopoulou G (2012) Somatostatin receptor expression in non-medullary thyroid carcinomas. Hormones (Athens) 11:290-296.

Pedraza-Arévalo S, Córdoba-Chacón J, Pozo-Salas AI, L-López F, de Lecea L, Gahete MD, Castaño JP, and Luque RM (2015) Not so giants: mice lacking both somatostatin and cortistatin have high GH levels but show no changes in growth rate or IGF-1 levels. Endocrinology 156:1958-1964

Pedraza-Arévalo S, Hormaechea-Agulla D, Gómez-Gómez E, Requena MJ, Selth LA Gahete MD, Castaño JP, and Luque RM (2017) Somatostatin receptor subtype 1 as a potential diagnostic marker and therapeutic target in prostate cancer. Prostate 77:1499-1511.

Peineau S, Guimiot F, Csaba Z, Jacquier S, Fafouri A, Schwendimann L, de Roux N, Schulz S, Gressens P, Auvin S, et al. (2014) Somatostatin receptors type 2 and 5 expression and localization during human pituitary development. Endocrinology 155:33-39.

Peineau S, Potier B, Petit F, Dournaud P, Epelbaum J, and Gardette R (2003) AMPA sst2 somatostatin receptor interaction in rat hypothalamus requires activation of NMDA and/or metabotropic glutamate receptors and depends on intracellular calcium. J Physiol 546:101-117.

Pérez J and Hoyer D (1995) Co-expression of somatostatin SSTR-3 and SSTR-4 receptor messenger RNAs in the rat brain. Neuroscience 64:241-253.

Pérez J, Rigo M, Kaupmann K, Bruns C, Yasuda K, Bell GI, Lübbert H, and Hoyer D (1994) Localization of somatostatin (SRIF) SSTR-1, SSTR-2 and SSTR-3 receptor mRNA in rat brain by in situ hybridization. Naunyn Schmiedebergs Arch Phar macol 349:145-160.

Perez J, Viollet C, Doublier S, Videau C, Epelbaum J, and Baud L (2003) Somatostatin binds to murine macrophages through two distinct subsets of receptors. $J$ Neuroimmunol 138:38-44.

Petersenn S, Rasch AC, Böhnke C, and Schulte HM (2002a) Identification of an upstream pituitary-active promoter of human somatostatin receptor subtype 5 . Endocrinology 143:2626-2634.

Petersenn S, Rasch AC, Presch S, Beil FU, and Schulte HM (2002b) Characterization of the human somatostatin receptor type 4 promoter. Mol Cell Endocrinol 188 $75-83$.

Petrich A, Mann A, Kliewer A, Nagel F, Strigli A, Märtens JC, Pöll F, and Schulz S (2013) Phosphorylation of threonine 333 regulates trafficking of the human sst5 somatostatin receptor. Mol Endocrinol 27:671-682.

Peverelli E, Busnelli M, Vitali E, Giardino E, Galés C, Lania AG, Beck-Peccoz P, Chini B, Mantovani G, and Spada A (2013) Specific roles of G(i) protein family members revealed by dissecting SST5 coupling in human pituitary cells. J Cell Sci 126:638-644.

Peverelli E, Giardino E, Treppiedi D, Meregalli M, Belicchi M, Vaira V, Corbetta S, Verdelli C, Verrua E, Serban AL, et al. (2017) Dopamine receptor type 2 (DRD2) and somatostatin receptor type 2 (SSTR2) agonists are effective in inhibiting proliferation of progenitor/stem-like cells isolated from nonfunctioning pituitary tumors. Int $J$ Cancer 140:1870-1880.

Peverelli E, Giardino E, Treppiedi D, Vitali E, Cambiaghi V, Locatelli M, Lasio GB, Spada A, Lania AG, and Mantovani G (2014) Filamin A (FLNA) plays an essential role in somatostatin receptor 2 (SST2) signaling and stabilization after agonist stimulation in human and rat somatotroph tumor cells. Endocrinology 155:2932-2941.

Peverelli E, Lania AG, Mantovani G, Beck-Peccoz P, and Spada A (2009) Characterization of intracellular signaling mediated by human somatostatin receptor 5 : role of the DRY motif and the third intracellular loop. Endocrinology 150: 3169-3176.

Peverelli E, Mantovani G, Calebiro D, Doni A, Bondioni S, Lania A, Beck-Peccoz P, and Spada A (2008) The third intracellular loop of the human somatostatin receptor 5 is crucial for arrestin binding and receptor internalization after somatostatin stimulation. Mol Endocrinol 22:676-688.

Pfeiffer M, Koch T, Schröder H, Klutzny M, Kirscht S, Kreienkamp H-J, Höllt V, and Schulz S (2001) Homo- and heterodimerization of somatostatin receptor subtypes: inactivation of $\operatorname{sst}(3)$ receptor function by heterodimerization with $\operatorname{sst}(2 \mathrm{~A}) . J$ Biol Chem 276:14027-14036.

Pfeiffer M, Koch T, Schröder H, Laugsch M, Höllt V, and Schulz S (2002) Heterodimerization of somatostatin and opioid receptors cross-modulates phosphorylation, internalization, and desensitization. J Biol Chem 277:19762-19772.

Pilichowska M, Kimura N, Schindler M, Suzuki A, Yoshida R, and Nagura H (2000) Expression of somatostatin type 2A receptor correlates with estrogen receptor in human breast carcinoma. Endocr Pathol 11:57-67.

Pintér E, Helyes Z, and Szolcsányi J (2006) Inhibitory effect of somatostatin on inflammation and nociception. Pharmacol Ther 112:440-456.

Pisarek H, Krupiński R, Kubiak R, Borkowska E, Pawlikowski M, and Winczyk K (2011) Differential expression of somatostatin receptor subtype-related genes and proteins in non-functioning and functioning adrenal cortex adenomas. Mol Med Rep 4:963-969.

Pita-Gutierrez F, Pertega-Diaz S, Pita-Fernandez S, Pena L, Lugo G, SangiaoAlvarellos S, and Cordido F (2013) Place of preoperative treatment of acromegaly with somatostatin analog on surgical outcome: a systematic review and metaanalysis. PLoS One 8:e61523.

Pittaluga A, Feligioni M, Longordo F, Arvigo M, and Raiteri M (2005) Somatostatininduced activation and up-regulation of $\mathrm{N}$-methyl-D-aspartate receptor function mediation through calmodulin-dependent protein kinase II, phospholipase C, protein kinase $\mathrm{C}$, and tyrosine kinase in hippocampal noradrenergic nerve endings. $J$ Pharmacol Exp Ther 313:242-249.

Piwko C, Thoss VS, Probst A, and Hoyer D (1996) Localization and pharmacological characterization of somatostatin recognition sites in the human cerebellum. Neuropharmacology 35:713-723.

Piwko C, Thoss VS, Probst A, and Hoyer D (1997) The elusive nature of cerebellar somatostatin receptors: studies in rat, monkey and human cerebellum. $J$ Recept Signal Transduct Res 17:385-405.

Plöckinger U, Hoffmann U, Geese M, Lupp A, Buchfelder M, Flitsch J, Vajkoczy P, Jakob W, Saeger W, Schulz S, et al. (2012) DG3173 (somatoprim), a unique somatostatin receptor subtypes 2-, 4- and 5-selective analogue, effectively reduces GH secretion in human GH-secreting pituitary adenomas even in Octreotide nonresponsive tumours. Eur J Endocrinol 166:223-234.

Poitout L, Roubert P, Contour-Galcéra M-O, Moinet C, Lannoy J, Pommier J, Plas P, Bigg D, and Thurieau C (2001) Identification of potent non-peptide somatostatin antagonists with sst(3) selectivity. J Med Chem 44:2990-3000.

Pola S, Cattaneo MG, and Vicentini LM (2003) Anti-migratory and anti-invasive effect of somatostatin in human neuroblastoma cells: involvement of Rac and MAP kinase activity. J Biol Chem 278:40601-40606.

Pöll F, Doll C, and Schulz S (2011) Rapid dephosphorylation of G protein-coupled receptors by protein phosphatase $1 \beta$ is required for termination of $\beta$-arrestindependent signaling. J Biol Chem 286:32931-32936.

Pöll F, Lehmann D, Illing S, Ginj M, Jacobs S, Lupp A, Stumm R, and Schulz S (2010) Pasireotide and octreotide stimulate distinct patterns of sst2A somatostatin receptor phosphorylation. Mol Endocrinol 24:436-446.

Portela-Gomes GM, Stridsberg M, Grimelius L, Oberg K, and Janson ET (2000) Expression of the five different somatostatin receptor subtypes in endocrine cells of the pancreas. Appl Immunohistochem Mol Morphol 8:126-132.

Portela-Gomes GM, Stridsberg M, Grimelius L, Rorstad O, and Janson ET (2007) Differential expression of the five somatostatin receptor subtypes in human benign and malignant insulinomas: predominance of receptor subtype 4. Endocr Pathol 18:79-85.

Potorac I, Petrossians P, Daly AF, Schillo F, Ben Slama C, Nagi S, Sahnoun M, Brue T, Girard N, Chanson P, et al. (2015) Pituitary MRI characteristics in 297 acromegaly patients based on T2-weighted sequences. Endocr Relat Cancer 22:169-177.

Pradayrol L, Jörnvall H, Mutt V, and Ribet A (1980) N-terminally extended somatostatin: the primary structure of somatostatin-28. FEBS Lett 109:55-58.

Prevot TD, Gastambide F, Viollet C, Henkous N, Martel G, Epelbaum J, Beracochea D, and Guillou JL (2017) Roles of hippocampal somatostatin receptor subtypes in stress response and emotionality. Neuropsychopharmacology 42:1647-1656.

Pscherer A, Dörflinger U, Kirfel J, Gawlas K, Rüschoff J, Buettner R, and Schüle R (1996) The helix-loop-helix transcription factor SEF-2 regulates the activity of a novel initiator element in the promoter of the human somatostatin receptor II gene. EMBO J 15:6680-6690.

Puente E, Saint-Laurent N, Torrisani J, Furet C, Schally AV, Vaysse N, Buscail L, and Susini C (2001) Transcriptional activation of mouse sst2 somatostatin receptor promoter by transforming growth factor-beta: involvement of Smad4. J Biol Chem 276:13461-13468.

Puig Domingo M (2015) Treatment of acromegaly in the era of personalized and predictive medicine. Clin Endocrinol (Oxf) 83:3-14.

Puig-Domingo M, Luque RM, Reverter JL, López-Sánchez LM, Gahete MD, Culler MD, Díaz-Soto G, Lomeña F, Squarcia M, Mate JL, et al. (2014) The truncated isoform of somatostatin receptor5 (sst5TMD4) is associated with poorly differentiated thyroid cancer. PLoS One 9:e85527.

Puig-Domingo M, Resmini E, Gomez-Anson B, Nicolau J, Mora M, Palomera E, Martí C, Halperin I, and Webb SM (2010) Magnetic resonance imaging as a predictor of response to somatostatin analogs in acromegaly after surgical failure. $\mathrm{J}$ Clin Endocrinol Metab 95:4973-4978.

Qian ZR, Li T, Ter-Minassian M, Yang J, Chan JA, Brais LK, Masugi Y, Thiaglingam A, Brooks N, Nishihara R, et al. (2016) Association between somatostatin receptor expression and clinical outcomes in neuroendocrine tumors. Pancreas $\mathbf{4 5}$ 1386-1393.

Qiu C, Suzuki C, Zeyda T, Hochgeschwender U, de Lecea L, and Tallent MK (2005) Increased Seizure Severity and Reduced Somatostatin Effects in Hippocampus of Somatostatin Receptor Subtype 4 (SST4) Knock-Out Mice (Abstract 607.11), Society for Neuroscience, Washington, DC. 
Qiu C, Zeyda T, Johnson B, Hochgeschwender U, de Lecea L, and Tallent MK (2008) Somatostatin receptor subtype 4 couples to the M-current to regulate seizures. $J$ Neurosci 28:3567-3576.

Qiu CZ, Wang C, Huang ZX, Zhu SZ, Wu YY, and Qiu JL (2006) Relationship between somatostatin receptor subtype expression and clinicopathology, Ki-67, Bcl-2 and p53 in colorectal cancer. World J Gastroenterol 12:2011-2015.

Radojevic V and Bodmer D (2014) Expression and localization of somatostatin receptor types 3, 4 and 5 in the wild-type, SSTR1 and SSTR1/SSTR2 knockout mouse cochlea. Cell Tissue Res 358:717-727.

Rai U, Thrimawithana TR, Valery C, and Young SA (2015) Therapeutic uses of somatostatin and its analogues: current view and potential applications. Pharmacol Ther 152:98-110.

Ramírez JL, Grant M, Norman M, Wang XP, Moldovan S, de Mayo FJ, Brunicardi C, and Kumar U (2004) Deficiency of somatostatin (SST) receptor type 5 (SSTR5) is associated with sexually dimorphic changes in the expression of SST and SST receptors in brain and pancreas. Mol Cell Endocrinol 221:105-119.

Ramírez JL, Watt HL, Rocheville M, and Kumar U (2005) Agonist-induced up-regulation of human somatostatin receptor type 1 is regulated by betaarrestin-1 and requires an essential serine residue in the receptor C-tail. Biochim Biophys Acta 1669:182-192.

Raynor K, Lucki I, and Reisine T (1993a) Somatostatin receptors in the nucleus accumbens selectively mediate the stimulatory effect of somatostatin on locomotor activity in rats. J Pharmacol Exp Ther 265:67-73.

Raynor K, Murphy WA, Coy DH, Taylor JE, Moreau JP, Yasuda K, Bell GI, and Reisine T (1993b) Cloned somatostatin receptors: identification of subtypeselective peptides and demonstration of high affinity binding of linear peptides. Mol Pharmacol 43:838-844.

Reardon DB, Dent P, Wood SL, Kong T, and Sturgill TW (1997) Activation in vitro of somatostatin receptor subtypes 2,3 , or 4 stimulates protein tyrosine phosphatase activity in membranes from transfected Ras-transformed NIH 3T3 cells: coexpression with catalytically inactive SHP-2 blocks responsiveness. Mol Endocrinol 11:1062-1069

Redmann A, Rasch A, Tourne H, Mann K, and Petersenn S (2007) Characterization and transcriptional regulation of the human somatostatin receptor subtype 1 gene. Horm Metab Res 39:359-365.

Reichlin S (1983a) Somatostatin. N Engl J Med 309:1495-1501.

Reichlin S (1983b) Somatostatin (second of two parts). N Engl J Med 309:1556-1563. Reisine T and Bell GI (1995) Molecular biology of somatostatin receptors. Endocr Rev 16:427-442.

Remke M, Hering E, Gerber NU, Kool M, Sturm D, Rickert CH, Gerss J, Schulz S, Hielscher T, Hasselblatt M, et al. (2013) Somatostatin receptor subtype 2 (sst(2)) is a potential prognostic marker and a therapeutic target in medulloblastoma. Childs Nerv Syst 29:1253-1262.

Ren SG, Taylor J, Dong J, Yu R, Culler MD, and Melmed S (2003) Functional association of somatostatin receptor subtypes 2 and 5 in inhibiting human growth hormone secretion. J Clin Endocrinol Metab 88:4239-4245.

Rens-Domiano S, Law SF, Yamada Y, Seino S, Bell GI, and Reisine T (1992) Pharmacological properties of two cloned somatostatin receptors. Mol Pharmacol 42 $28-34$

Reubi JC, Eisenwiener K-P, Rink H, Waser B, and Mäcke HR (2002) A new peptidic somatostatin agonist with high affinity to all five somatostatin receptors. Eur $J$ Pharmacol 456:45-49.

Reubi JC, Laissue JA, Waser B, Steffen DL, Hipkin RW, and Schonbrunn A (1999) Immunohistochemical detection of somatostatin sst2a receptors in the lymphatic smooth muscular, and peripheral nervous systems of the human gastrointestinal tract: facts and artifacts. J Clin Endocrinol Metab 84:2942-2950.

Reubi JC, Schaer JC, Markwalder R, Waser B, Horisberger U, and Laissue J (1997) Distribution of somatostatin receptors in normal and neoplastic human tissues: recent advances and potential relevance. Yale J Biol Med 70:471-479.

Reubi JC, Schaer J-C, Wenger S, Hoeger C, Erchegyi J, Waser B, and Rivier J (2000a) $\mathrm{SST}_{3}$-selective potent peptidic somatostatin receptor antagonists. Proc Natl Acad Sci USA 97:13973-13978.

Reubi JC, Waser B, Liu Q, Laissue JA, and Schonbrunn A (2000b) Subcellular distribution of somatostatin sst2A receptors in human tumors of the nervous and neuroendocrine systems: membranous versus intracellular location. J Clin Endocrinol Metab 85:3882-3891.

Reubi JC, Waser B, Schaer J-C, and Laissue JA (2001) Somatostatin receptor sst1sst5 expression in normal and neoplastic human tissues using receptor autoradiography with subtype-selective ligands. Eur J Nucl Med 28:836-846.

Reynaert H, Rombouts K, Jia Y, Urbain D, Chatterjee N, Uyama N, and Geerts A (2005) Somatostatin at nanomolar concentration reduces collagen I and III synthesis by, but not proliferation of activated rat hepatic stellate cells. $\mathrm{Br} J$ Pharmacol 146:77-88.

Reynaert H, Rombouts K, Vandermonde A, Urbain D, Kumar U, Bioulac-Sage P, Pinzani M, Rosenbaum J, and Geerts A (2004) Expression of somatostatin receptors in normal and cirrhotic human liver and in hepatocellular carcinoma. Gut $\mathbf{5 3}$ 1180-1189

Riaz H, Liang A, Khan MK, Dong P, Han L, Shahzad M, Chong Z, Ahmad S, Hua G and Yang L (2013) Somatostatin and its receptors: functional regulation in the development of mice Sertoli cells. I Steroid Biochem Mol Biol 138:257-266.

Righi L, Volante M, Tavaglione V, Billè A, Daniele L, Angusti T, Inzani F, Pelosi G, Rindi G, and Papotti M (2010) Somatostatin receptor tissue distribution in lung neuroendocrine tumours: a clinicopathologic and immunohistochemical study of 218 'clinically aggressive' cases. Ann Oncol 21:548-555.

Rinke A, Müller H-H, Schade-Brittinger C, Klose K-J, Barth P, Wied M, Mayer C, Aminossadati B, Pape U-F, Bläker M, et al.; PROMID Study Group (2009) Placebocontrolled, double-blind, prospective, randomized study on the effect of octreotide LAR in the control of tumor growth in patients with metastatic neuroendocrine midgut tumors: a report from the PROMID Study Group. J Clin Oncol 27:4656-4663.

Ritvonen E, Pitkänen E, Karppinen A, Vehkavaara S, Demir H, Paetau A, SchalinJäntti C, and Karhu A (2017) Impact of AIP and inhibitory G protein alpha
2 proteins on clinical features of sporadic GH-secreting pituitary adenomas. Eur $J$ Endocrinol 176:243-252.

Rocheville M, Lange DC, Kumar U, Patel SC, Patel RC, and Patel YC (2000a) Receptors for dopamine and somatostatin: formation of hetero-oligomers with enhanced functional activity. Science 288:154-157.

Rocheville M, Lange DC, Kumar U, Sasi R, Patel RC, and Patel YC (2000b) Subtypes of the somatostatin receptor assemble as functional homo- and heterodimers. J Biol Chem 275:7862-7869.

Rohrer L, Raulf F, Bruns C, Buettner R, Hofstaedter F, and Schüle R (1993) Cloning and characterization of a fourth human somatostatin receptor. Proc Natl Acad Sci USA 90:4196-4200.

Rohrer SP, Birzin ET, Mosley RT, Berk SC, Hutchins SM, Shen D-M, Xiong Y, Hayes EC, Parmar RM, Foor F, et al. (1998) Rapid identification of subtype-selective agonists of the somatostatin receptor through combinatorial chemistry. Science 282:737-740.

Roosterman D, Brune NEI, Kreuzer OJ, Feld M, Pauser S, Zarse K, Steinhoff M, and Meyerhof W (2008) Intracellular degradation of somatostatin-14 following somatostatin-receptor3-mediated endocytosis in rat insulinoma cells. FEBS J 275 4728-4739.

Roosterman D, Cottrell GS, Padilla BE, Muller L, Eckman CB, Bunnett NW, and Steinhoff M (2007) Endothelin-converting enzyme 1 degrades neuropeptides in endosomes to control receptor recycling. Proc Natl Acad Sci USA 104: 11838-11843.

Roosterman D, Glassmeier G, Baumeister H, Scherübl H, and Meyerhof W (1998) A somatostatin receptor 1 selective ligand inhibits $\mathrm{Ca} 2+$ currents in rat insulinoma 1046-38 cells. FEBS Lett 425:137-140

Roosterman D, Roth A, Kreienkamp HJ, Richter D, and Meyerhof W (1997) Distinct agonist-mediated endocytosis of cloned rat somatostatin receptor subtypes expressed in insulinoma cells. J Neuroendocrinol 9:741-751.

Rosenfeld MG (1991) POU-domain transcription factors: pou-er-ful developmental regulators. Genes Dev 5:897-907.

Roth A, Kreienkamp H-J, Meyerhof W, and Richter D (1997a) Phosphorylation of four amino acid residues in the carboxyl terminus of the rat somatostatin receptor subtype 3 is crucial for its desensitization and internalization. $J$ Biol Chem 272 . 23769-23774

Roth A, Kreienkamp HJ, Nehring RB, Roosterman D, Meyerhof W, and Richter D (1997b) Endocytosis of the rat somatostatin receptors: subtype discrimination, ligand specificity, and delineation of carboxy-terminal positive and negative sequence motifs. DNA Cell Biol 16:111-119.

Rubin J, Ajani J, Schirmer W, Venook AP, Bukowski R, Pommier R, Saltz L, Dandona P, and Anthony L (1999) Octreotide acetate long-acting formulation versus open-label subcutaneous octreotide acetate in malignant carcinoid syndrome. $J$ Clin Oncol 17:600-606.

Ruscica M, Arvigo M, Gatto F, Dozio E, Feltrin D, Culler MD, Minuto F, Motta M, Ferone D, and Magni P (2010) Regulation of prostate cancer cell proliferation by somatostatin receptor activation. Mol Cell Endocrinol 315:254-262.

Ruuska T, Ramirez Escalante Y, Vaittinen S, Gardberg M, Kiviniemi A, Marjamaki P, Kemppainen J, Jyrkkio S, and Minn H (2018) Somatostatin receptor expression in lymphomas: a source of false diagnosis of neuroendocrine tumor at (68)GaDOTANOC PET/CT imaging. Acta Oncol 57:283-289.

Sampedro-Núñez M, Luque RM, Ramos-Levi AM, Gahete MD, Serrano-Somavilla A Villa-Osaba A Adrados M, Ibáñez-Costa A, Martín-Pérez E, Culler MD, et al. (2016) Presence of sst5TMD4, a truncated splice variant of the somatostatin receptor subtype 5 , is associated to features of increased aggressiveness in pancreatic neuroendocrine tumors. Oncotarget 7:6593-6608.

Samson WK, Zhang JV, Avsian-Kretchmer O, Cui K, Yosten GLC, Klein C, Lyu R-M, Wang YX, Chen XQ, Yang J, et al. (2008) Neuronostatin encoded by the somatostatin gene regulates neuronal, cardiovascular, and metabolic functions. $J$ Biol Chem 283:31949-31959.

Sándor K, Elekes K, Szabó A, Pintér E, Engström M, Wurster S, Szolcsányi J, and Helyes Z (2006) Analgesic effects of the somatostatin sst4 receptor selective agonist J-2156 in acute and chronic pain models. Eur J Pharmacol 539:71-75.

Sandoval KE, Farr SA, Banks WA, Crider AM, Morley JE, and Witt KA (2012 Somatostatin receptor subtype-4 agonist NNC 26-9100 decreases extracellular and intracellular $\mathrm{A} \beta_{1-42}$ trimers. Eur J Pharmacol 683:116-124.

Sandoval KE, Farr SA, Banks WA, Crider AM, Morley JE, and Witt KA (2013) Somatostatin receptor subtype-4 agonist NNC 26-9100 mitigates the effect of soluble $\mathrm{A} \beta(42)$ oligomers via a metalloproteinase-dependent mechanism. Brain Res 1520: $145-156$

Santis S, Kastellakis A, Kotzamani D, Pitarokoili K, Kokona D, and Thermos K (2009) Somatostatin increases rat locomotor activity by activating sst(2) and sst (4) receptors in the striatum and via glutamatergic involvement. Naunyn Schmiedebergs Arch Pharmacol 379:181-189.

Saveanu A, Gunz G, Dufour H, Caron P, Fina F, Ouafik L, Culler MD, Moreau JP, Enjalbert A, and Jaquet P (2001) Bim-23244, a somatostatin receptor subtype 2 and 5 -selective analog with enhanced efficacy in suppressing growth hormone (GH) from octreotide-resistant human GH-secreting adenomas. J Clin Endocrinol Metab 86:140-145.

Saveanu A, Lavaque E, Gunz G, Barlier A, Kim S, Taylor JE, Culler MD, Enjalbert A and Jaquet P (2002) Demonstration of enhanced potency of a chimeric somatostatin-dopamine molecule, BIM-23A387, in suppressing growth hormone and prolactin secretion from human pituitary somatotroph adenoma cells. J Clin Endocrinol Metab 87:5545-5552.

Saveanu A, Muresan M, De Micco C, Taieb D, Germanetti AL, Sebag F, Henry JF, Brunaud L, Enjalbert A, Weryha G, et al. (2011) Expression of somatostatin receptors, dopamine $\mathrm{D}_{2}$ receptors, noradrenaline transporters, and vesicular monoamine transporters in 52 pheochromocytomas and paragangliomas. Endocr Relat Cancer 18:287-300.

Scheich B, Csekő K, Borbély É, Ábrahám I, Csernus V, Gaszner B, and Helyes Z (2017) Higher susceptibility of somatostatin 4 receptor gene-deleted mice to chronic stressinduced behavioral and neuroendocrine alterations. Neuroscience 346:320-336. 
Scheich B, Gaszner B, Kormos V, László K, Ádori C, Borbély É, Hajna Z, Tékus V, Bölcskei K, Ábrahám I, et al. (2016) Somatostatin receptor subtype 4 activation is involved in anxiety and depression-like behavior in mouse models. Neuropharmacology 101:204-215.

Schindler M, Harrington KA, Humphrey PPA, and Emson PC (1995) Cellular localisation and co-expression of somatostatin receptor messenger RNAs in the human brain. Brain Res Mol Brain Res 34:321-326.

Schindler M, Holloway S, Hathway G, Woolf CJ, Humphrey PP, and Emson PC (1998a) Identification of somatostatin sst2(a) receptor expressing neurones in central regions involved in nociception. Brain Res 798:25-35.

Schindler M, Holloway S, Humphrey PP, Waldvogel H, Faull RL, Berger W, and Emson PC (1998b) Localization of the somatostatin sst2(a) receptor in human cerebral cortex, hippocampus and cerebellum. Neuroreport 9:521-525.

Schindler M, Humphrey PP, Löhrke S, and Friauf E (1999) Immunohistochemical localization of the somatostatin sst2(b) receptor splice variant in the rat central nervous system. Neuroscience 90:859-874.

Schindler M, Sellers LA, Humphrey PP, and Emson PC (1997) Immunohistochemical localization of the somatostatin SST2(A) receptor in the rat brain and spinal cord. Neuroscience 76:225-240.

Schmid HA (2008) Pasireotide (SOM230): development, mechanism of action and potential applications. Mol Cell Endocrinol 286:69-74.

Schoeffter P, Pérez J, Langenegger D, Schüpbach E, Bobirnac I, Lübbert H, Bruns C, and Hoyer D (1995) Characterization and distribution of somatostatin SS-1 and SRIF-1 binding sites in rat brain: identity with SSTR-2 receptors. Eur J Pharmacol 289:163-173.

Schottelius M, Poethko T, Herz M, Reubi J-C, Kessler H, Schwaiger M, and Wester H-J (2004) First (18)F-labeled tracer suitable for routine clinical imaging of sst receptor-expressing tumors using positron emission tomography. Clin Cancer Res 10:3593-3606.

Schottelius M, Šimeček J, Hoffmann F, Willibald M, Schwaiger M, and Wester H-J (2015) Twins in spirit - episode I: comparative preclinical evaluation of $[(68) \mathrm{Ga}]$ DOTATATE and [(68)Ga]HA-DOTATATE. EJNMMI Res 5:22.

Schreff M, Schulz S, Händel M, Keilhoff G, Braun H, Pereira G, Klutzny M, Schmidt H, Wolf G, and Höllt V (2000) Distribution, targeting, and internalization of the sst4 somatostatin receptor in rat brain. J Neurosci 20:3785-3797.

Schuelert N, Just S, Kuelzer R, Corradini L, Gorham LCJ, and Doods H (2015) The somatostatin receptor 4 agonist J-2156 reduces mechanosensitivity of peripheral nerve afferents and spinal neurons in an inflammatory pain model. Eur $J$ Pharmacol 746:274-281.

Schulz S, Bosquet C, Castaño JP, Culler M, Epelbaum J, Hofland L, Hoyer D, Reubi J, Schmid H, Schonbrunn A, et al. (2017) Somatostatin receptors, in IUPHAR/BPS Guide to Pharmacology.

Schulz S, Händel M, Schreff M, Schmidt H, and Höllt V (2000a) Localization of five somatostatin receptors in the rat central nervous system using subtype-specific antibodies. J Physiol Paris 94:259-264.

Schulz S, Lehmann A, Kliewer A, and Nagel F (2014) Fine-tuning somatostatin receptor signalling by agonist-selective phosphorylation and dephosphorylation: IUPHAR review 5. Br J Pharmacol 171:1591-1599.

Schulz S, Pauli SU, Schulz S, Handel M, Dietzmann K, Firsching R, and Hollt V (2000b) Immunohistochemical determination of five somatostatin receptors in meningioma reveals frequent overexpression of somatostatin receptor subtype sst2A. Clin Cancer Res 6:1865-1874.

Schulz S, Schmidt H, Händel M, Schreff M, and Höllt V (1998a) Differential distribution of alternatively spliced somatostatin receptor 2 isoforms (sst2A and sst2B) in rat spinal cord. Neurosci Lett 257:37-40.

Schulz S, Schmitt J, and Weise W (2003) Frequent expression of immunoreactive somatostatin receptors in cervical and endometrial cancer. Gynecol Oncol 89 385-390.

Schulz S, Schreff M, Schmidt H, Händel M, Przewlocki R, and Höllt V (1998b) Immunocytochemical localization of somatostatin receptor sst2A in the rat spinal cord and dorsal root ganglia. Eur J Neurosci 10:3700-3708.

Schulz S, Schulz S, Schmitt J, Wiborny D, Schmidt H, Olbricht S, Weise W, Roessner A, Gramsch C, and Hollt V (1998c) Immunocytochemical detection of somatostatin receptors sst1, sst2A, sst2B, and sst3 in paraffin-embedded breast cancer tissue using subtype-specific antibodies. Clin Cancer Res 4:2047-2052.

Schwabe W, Brennan MB, and Hochgeschwender U (1996) Isolation and characterization of the mouse (Mus musculus) somatostatin receptor type-4-encoding gene (mSSTR4). Gene 168:233-235.

Schweitzer P, Madamba S, and Siggins GR (1990) Arachidonic acid metabolites as mediators of somatostatin-induced increase of neuronal M-current. Nature $\mathbf{3 4 6}$ 464-467.

Schweitzer P, Madamba SG, and Siggins GR (1998) Somatostatin increases a voltage-insensitive $\mathrm{K}+$ conductance in rat CA1 hippocampal neurons. J Neurophysiol 79:1230-1238.

Schweitzer P, Madamba SG, and Siggins GR (2003) The sleep-modulating peptide cortistatin augments the h-current in hippocampal neurons. $J$ Neurosci 23: 10884-10891.

Sedqi M, Roy S, Mohanraj D, Ramakrishnan S, and Loh HH (1996) Activation of rat thymocytes selectively upregulates the expression of somatostatin receptor subtype-1. Biochem Mol Biol Int 38:103-112.

Segond von Banchet G, Schindler M, Hervieu GJ, Beckmann B, Emson PC, and Heppelmann B (1999) Distribution of somatostatin receptor subtypes in rat lumbar spinal cord examined with gold-labelled somatostatin and anti-receptor antibodies. Brain Res 816:254-257.

Sellers LA, Feniuk W, Humphrey PPA, and Lauder H (1999) Activated G proteincoupled receptor induces tyrosine phosphorylation of STAT3 and agonist-selective serine phosphorylation via sustained stimulation of mitogen-activated protein kinase: resultant effects on cell proliferation. J Biol Chem 274:16423-16430.

Selmer I-S, Schindler M, Humphrey PPA, Waldvogel HJ, Faull RLM, and Emson PC (2000) First localisation of somatostatin sst(4) receptor protein in selected human brain areas: an immunohistochemical study. Brain Res Mol Brain Res 82:114-125.
Señarís RM, Lago F, and Diéguez C (1996) Gonadal regulation of somatostatin receptor 1, 2 and 3 mRNA levels in the rat anterior pituitary. Brain Res Mol Brain Res 38:171-175.

Shah SK, He S, Guo L, Truong Q, Qi H, Du W, Lai Z, Liu J, Jian T, Hong Q, et al. (2015) Discovery of MK-1421, a potent, selective sstr3 antagonist, as a development candidate for type 2 diabetes. ACS Med Chem Lett 6:513-517.

Sharma K, Patel YC, and Srikant CB (1996) Subtype-selective induction of wild-type p53 and apoptosis, but not cell cycle arrest, by human somatostatin receptor 3 . Mol Endocrinol 10:1688-1696.

Shen LP and Rutter WJ (1984) Sequence of the human somatostatin I gene. Science 224:168-171.

Shen M, Shou X, Wang Y, Zhang Z, Wu J, Mao Y, Li S, and Zhao Y (2010) Effect of presurgical long-acting octreotide treatment in acromegaly patients with invasive pituitary macroadenomas: a prospective randomized study. Endocr $J \mathbf{5 7}$ $1035-1044$

Shen Z, Chen X, Li Q, Zhou C, Li J, Ye H, and Duan S (2016) SSTR2 promoter hypermethylation is associated with the risk and progression of laryngeal squamous cell carcinoma in males. Diagn Pathol 11:10.

Shi TJ, Xiang Q, Zhang MD, Barde S, Kai-Larsen Y, Fried K, Josephson A, Glück L, Deyev SM, Zvyagin AV, et al. (2014) Somatostatin and its $2 \mathrm{~A}$ receptor in dorsal root ganglia and dorsal horn of mouse and human: expression, trafficking and possible role in pain. Mol Pain 10:12.

Shimon I, Rubinek T, Hadani M, and Alhadef N (2004) PTR-3173 (somatoprim), a novel somatostatin analog with affinity for somatostatin receptors 2,4 and 5 is a potent inhibitor of human $\mathrm{GH}$ secretion. $J$ Endocrinol Invest 27:721-727.

Shimon I, Taylor JE, Dong JZ, Bitonte RA, Kim S, Morgan B, Coy DH, Culler MD, and Melmed S (1997a) Somatostatin receptor subtype specificity in human fetal pituitary cultures: differential role of SSTR2 and SSTR5 for growth hormone, thyroid-stimulating hormone, and prolactin regulation. J Clin Invest 99:789-798.

Shimon I, Yan X, Taylor JE, Weiss MH, Culler MD, and Melmed S (1997b) Somatostatin receptor (SSTR) subtype-selective analogues differentially suppress in vitro growth hormone and prolactin in human pituitary adenomas: novel potential therapy for functional pituitary tumors. J Clin Invest 100:2386-2392.

Siehler S and Hoyer D (1999a) Characterisation of human recombinant somatostatin receptors. 2. Modulation of GTPgammaS binding. Naunyn Schmiedebergs Arch Pharmacol 360:500-509.

Siehler S and Hoyer D (1999b) Characterisation of human recombinant somatostatin receptors. 3. Modulation of adenylate cyclase activity. Naunyn Schmiedebergs Arch Pharmacol 360:510-521.

Siehler S and Hoyer D (1999c) Characterisation of human recombinant somatostatin receptors. 4. Modulation of phospholipase C activity. Naunyn Schmiedebergs Arch Pharmacol 360:522-532.

Siehler S, Nunn C, Hannon J, Feuerbach D, and Hoyer D (2008) Pharmacological profile of somatostatin and cortistatin receptors. Mol Cell Endocrinol 286:26-34.

Siehler S, Seuwen K, and Hoyer D (1998) [125I][Tyr3]octreotide labels human somatostatin sst2 and sst5 receptors. Eur J Pharmacol 348:311-320.

Silverstein JM (2016) Hyperglycemia induced by pasireotide in patients with Cushing's disease or acromegaly. Pituitary 19:536-543.

Simo R and Hernandez C; European Consortium for the Early Treatment of Diabetic Retinopathy (EUROCONDOR) (2014) Neurodegeneration in the diabetic eye: new insights and therapeutic perspectives. Trends Endocrinol Metab 25:23-33.

Singh V, Brendel MD, Zacharias S, Mergler S, Jahr H, Wiedenmann B, Bretzel RG, Plöckinger U, and Strowski MZ (2007) Characterization of somatostatin receptor subtype-specific regulation of insulin and glucagon secretion: an in vitro study on isolated human pancreatic islets. J Clin Endocrinol Metab 92:673-680.

Sinisi AA, Bellastella A, Prezioso D, Nicchio MR, Lotti T, Salvatore M, and Pasquali D (1997) Different expression patterns of somatostatin receptor subtypes in cultured epithelial cells from human normal prostate and prostate cancer. J Clin Endocrinol Metab 82:2566-2569.

Smale ST, Schmidt MC, Berk AJ, and Baltimore D (1990) Transcriptional activation by $\mathrm{Sp} 1$ as directed through TATA or initiator: specific requirement for mammalian transcription factor IID. Proc Natl Acad Sci USA 87:4509-4513.

Smalley KSM, Feniuk W, and Humphrey PPA (1998) Differential agonist activity of somatostatin and L-362855 at human recombinant sst4 receptors. Br J Pharmacol 125:833-841.

Smalley KSM, Feniuk W, Sellers LA, and Humphrey PPA (1999) The pivotal role of phosphoinositide-3 kinase in the human somatostatin sst(4) receptor-mediated stimulation of p44/p42 mitogen-activated protein kinase and extracellular acidification. Biochem Biophys Res Commun 263:239-243.

Smalley KSM, Koenig JA, Feniuk W, and Humphrey PPA (2001) Ligand internalization and recycling by human recombinant somatostatin type 4 (h sst(4)) receptors expressed in CHO-K1 cells. Br J Pharmacol 132:1102-1110.

Smith PA (2009) N-type $\mathrm{Ca}(2+)$-channels in murine pancreatic beta-cells are inhibited by an exclusive coupling with somatostatin receptor subtype 1. Endocrinology 150:741-748.

Smith PA, Sellers LA, and Humphrey PP (2001) Somatostatin activates two types of inwardly rectifying $\mathrm{K}+$ channels in MIN-6 cells. $J$ Physiol 532:127-142.

Solinski HJ, Gudermann T, and Breit A (2014) Pharmacology and signaling of MASrelated G protein-coupled receptors. Pharmacol Rev 66:570-597.

Somvanshi RK, Billova S, Kharmate G, Rajput PS, and Kumar U (2009) C-tail mediated modulation of somatostatin receptor type-4 homo- and heterodimerizations and signaling. Cell Signal 21:1396-1414.

Song KB, Kim SC, Kim JH, Seo D-W, Hong S-M, Park K-M, Hwang DW, Lee JH, and Lee Y-J (2016) Prognostic value of somatostatin receptor subtypes in pancreatic neuroendocrine tumors. Pancreas 45:187-192.

Song P, Hu JY, and Zhao ZQ (2002) Spinal somatostatin SSTR2A receptors are preferentially up-regulated and involved in thermonociception but not mechanonociception. Exp Neurol 178:280-287.

Souza-Moreira L, Morell M, Delgado-Maroto V, Pedreño M, Martinez-Escudero L, Caro M, O'Valle F, Luque R, Gallo M, de Lecea L, et al. (2013) Paradoxical effect of cortistatin treatment and its deficiency on experimental autoimmune encephalomyelitis. J Immunol 191:2144-2154. 
Spier AD and de Lecea L (2000) Cortistatin: a member of the somatostatin neuropeptide family with distinct physiological functions. Brain Res Brain Res Rev $\mathbf{3 3}$ : $228-241$.

Srikant CB and Shen SH (1996) Octapeptide somatostatin analog SMS 201-995 induces translocation of intracellular PTP1C to membranes in MCF-7 human breast adenocarcinoma cells. Endocrinology 137:3461-3468.

Srirajaskanthan R, Watkins J, Marelli L, Khan K, and Caplin ME (2009) Expression of somatostatin and dopamine 2 receptors in neuroendocrine tumours and the potential role for new biotherapies. Neuroendocrinology 89:308-314

Stanić D, Malmgren H, He H, Scott L, Aperia A, and Hökfelt T (2009) Developmental changes in frequency of the ciliary somatostatin receptor 3 protein. Brain Res $\mathbf{1 2 4 9}$ 101-112.

Steinberg F, Gallon M, Winfield M, Thomas EC, Bell AJ, Heesom KJ, Tavaré JM, and Cullen PJ (2013) A global analysis of SNX27-retromer assembly and cargo specificity reveals a function in glucose and metal ion transport. Nat Cell Biol 15: 461-471.

Stengel A, Goebel-Stengel M, Wang L, Larauche M, Rivier J, and Taché Y (2011) Central somatostatin receptor 1 activation reverses acute stress-related alterations of gastric and colonic motor function in mice. Neurogastroenterol Motil 23: e223-e236.

Stengel A, Karasawa H, and Taché Y (2015) The role of brain somatostatin receptor 2 in the regulation of feeding and drinking behavior. Horm Behav 73:15-22.

Stengel A, Rivier J, and Taché Y (2013) Modulation of the adaptive response to stress by brain activation of selective somatostatin receptor subtypes. Peptides 42:70-77.

Stengel A and Taché YF (2017) Activation of brain somatostatin signaling suppresses CRF receptor-mediated stress response. Front Neurosci 11:231.

Sternini C, Wong H, Wu SV, de Giorgio R, Yang M, Reeve J Jr, Brecha NC, and Walsh JH (1997) Somatostatin 2A receptor is expressed by enteric neurons, and by interstitial cells of Cajal and enterochromaffin-like cells of the gastrointestinal tract. J Comp Neurol 386:396-408.

Stollberg S, Kämmerer D, Neubauer E, Schulz S, Simonitsch-Klupp I, Kiesewetter B, Raderer M, and Lupp A (2016) Differential somatostatin and CXCR4 chemokine receptor expression in MALT-type lymphoma of gastric and extragastric origin. $J$ Cancer Res Clin Oncol 142:2239-2247.

Stolz B, Weckbecker G, Smith-Jones PM, Albert R, Raulf F, and Bruns C (1998) The somatostatin receptor-targeted radiotherapeutic [90Y-DOTA-DPhe1, Tyr3] octreotide (90Y-SMT 487) eradicates experimental rat pancreatic CA 20948 tumours. Eur J Nucl Med 25:668-674.

Stroh T, Jackson AC, Dal Farra C, Schonbrunn A, Vincent JP, and Beaudet A (2000a) Receptor-mediated internalization of somatostatin in rat cortical and hippocampal neurons. Synapse 38:177-186.

Stroh T, Jackson AC, Sarret P, Dal Farra C, Vincent JP, Kreienkamp HJ, Mazella J, and Beaudet A (2000b) Intracellular dynamics of sst5 receptors in transfected COS-7 cells: maintenance of cell surface receptors during ligand-induced endocytosis. Endocrinology 141:354-365.

Stroh T, Kreienkamp HJ, and Beaudet A (1999) Immunohistochemical distribution of the somatostatin receptor subtype 5 in the adult rat brain: predominant expression in the basal forebrain. $J$ Comp Neurol 412:69-82.

Stroh T, Sarret P, Tannenbaum GS, and Beaudet A (2006) Immunohistochemical distribution and subcellular localization of the somatostatin receptor subtype 1 (sst1) in the rat hypothalamus. Neurochem Res 31:247-257.

Strosberg J, El-Haddad G, Wolin E, Hendifar A, Yao J, Chasen B, Mittra E, Kunz PL, Kulke MH, Jacene H, et al.; NETTER-1 Trial Investigators (2017) Phase 3 trial of ${ }^{177} \mathrm{Lu}$-dotatate for midgut neuroendocrine tumors. N Engl J Med 376:125-135.

Strowski MZ and Blake AD (2008) Function and expression of somatostatin receptors of the endocrine pancreas. Mol Cell Endocrinol 286:169-179.

Strowski MZ, Kohler M, Chen HY, Trumbauer ME, Li Z, Szalkowski D, Gopal-Truter S, Fisher JK, Schaeffer JM, Blake AD, et al. (2003) Somatostatin receptor subtype 5 regulates insulin secretion and glucose homeostasis. Mol Endocrinol 17:93-106.

Strowski MZ, Parmar RM, Blake AD, and Schaeffer JM (2000) Somatostatin inhibits insulin and glucagon secretion via two receptors subtypes: an in vitro study of pancreatic islets from somatostatin receptor 2 knockout mice. Endocrinology 141 $111-117$.

Stumm RK, Zhou C, Schulz S, Endres M, Kronenberg G, Allen JP, Tulipano G, and Höllt V (2004) Somatostatin receptor 2 is activated in cortical neurons and contributes to neurodegeneration after focal ischemia. $J$ Neurosci 24:11404-11415.

Sun X, Haley J, Bulgakov OV, Cai X, McGinnis J, and Li T (2012) Tubby is required for trafficking $\mathrm{G}$ protein-coupled receptors to neuronal cilia. Cilia 1:21.

Szafran K, Lukasiewicz S, Faron-Gorecka A, Kolasa M, Kusmider M, Solich J, and Dziedzicka-Wasylewska M (2012) Antidepressant drugs promote the heterodimerization of the dopamine D2 and somatostatin Sst5 receptors-fluorescence in vitro studies. Pharmacol Rep 64:1253-1258.

Szafran-Pilch K, Faron-Górecka A, Kolasa M, Żurawek D, Szlachta M, Solich J, Kuśmider M, and Dziedzicka-Wasylewska M (2017) Antidepressants promote formation of heterocomplexes of dopamine D2 and somatostatin subtype 5 receptors in the mouse striatum. Brain Res Bull 135:92-97.

Takeda J, Fernald AA, Yamagata K, Le Beau MM, and Bell GI (1995) Localization of human somatostatin receptor 5 gene (SSTR5) to chromosome band $16 \mathrm{p} 13.3$ by fluorescence in situ hybridization. Genomics 26:638-639.

Takei M, Suzuki M, Kajiya H, Ishii Y, Tahara S, Miyakoshi T, Egashira N, Takekoshi S, Sanno N, Teramoto A, et al. (2007) Immunohistochemical detection of somatostatin receptor (SSTR) subtypes $2 \mathrm{~A}$ and 5 in pituitary adenoma from acromegalic patients: good correlation with preoperative response to octreotide. Endocr Pathol 18:208-216.

Tallent M, Liapakis G, O'Carroll AM, Lolait SJ, Dichter M, and Reisine T (1996) Somatostatin receptor subtypes SSTR2 and SSTR5 couple negatively to an L-type Ca2+ current in the pituitary cell line AtT-20. Neuroscience 71:1073-1081.

Tallent MK, Patterson C, Hon B, Qiu C, Zeyda T, Hochgeschwender U, and de Lecea L (2005) Somatostatin Receptor Subtype 3 Is Critical for Hippocampal-Dependent Memory: Studies in SST3 Receptor Knock-Out Mice (Abstract 607.10), Society for Neuroscience, Washington, DC.
Tallent MK and Qiu C (2008) Somatostatin: an endogenous antiepileptic. Mol Cell Endocrinol 286:96-103.

Tallent MK and Siggins GR (1997) Somatostatin depresses excitatory but not inhibitory neurotransmission in rat CA1 hippocampus. $J$ Neurophysiol $\mathbf{7 8}$ : 3008-3018.

Tallent MK and Siggins GR (1999) Somatostatin acts in CA1 and CA3 to reduce hippocampal epileptiform activity. J Neurophysiol 81:1626-1635.

Taniyama Y, Suzuki T, Mikami Y, Moriya T, Satomi S, and Sasano H (2005) Systemic distribution of somatostatin receptor subtypes in human: an immunohistochemical study. Endocr J 52:605-611.

Tannenbaum GS, Zhang WH, Lapointe M, Zeitler P, and Beaudet A (1998) Growth hormone-releasing hormone neurons in the arcuate nucleus express both Sst1 and Sst2 somatostatin receptor genes. Endocrinology 139:1450-1453.

Tateno T, Kato M, Tani Y, Oyama K, Yamada S, and Hirata Y (2009) Differential expression of somatostatin and dopamine receptor subtype genes in adrenocorticotropin (ACTH)-secreting pituitary tumors and silent corticotroph adenomas. Endocr $J$ 56:579-584.

Tatsi A, Maina T, Cescato R, Waser B, Krenning EP, de Jong M, Cordopatis P, Reubi JC, and Nock BA (2012) [111In-DOTA]Somatostatin-14 analogs as potentia pansomatostatin-like radiotracers: first results of a preclinical study. EJNMMI Res 2:25.

Theodoropoulou M and Stalla GK (2013) Somatostatin receptors: from signaling to clinical practice. Front Neuroendocrinol 34:228-252

Theodoropoulou M, Zhang J, Laupheimer S, Paez-Pereda M, Erneux C, Florio T, Pagotto U, and Stalla GK (2006) Octreotide, a somatostatin analogue, mediates its antiproliferative action in pituitary tumor cells by altering phosphatidylinositol 3-kinase signaling and inducing Zac1 expression. Cancer Res 66:1576-1582.

Thermos K, Bagnoli P, Epelbaum J, and Hoyer D (2006) The somatostatin sst1 receptor: an autoreceptor for somatostatin in brain and retina? Pharmacol Ther 110: 455-464.

Thoss VS, Duc D, and Hoyer D (1996a) Somatostatin receptors in the developing rat brain. Eur $J$ Pharmacol 297:145-155.

Thoss VS, Pérez J, Probst A, and Hoyer D (1996b) Expression of five somatostatin receptor mRNAs in the human brain and pituitary. Naunyn Schmiedebergs Arch Pharmacol 354:411-419.

Tirone TA, Norman MA, Moldovan S, DeMayo FJ, Wang XP, and Brunicardi FC (2003) Pancreatic somatostatin inhibits insulin secretion via SSTR-5 in the isolated perfused mouse pancreas model. Pancreas 26:e67-e73.

Torrisani J, Hanoun N, Laurell H, Lopez F, Maoret JJ, Souque A, Susini C, Cordelier $\mathrm{P}$, and Buscail L (2008) Identification of an upstream promoter of the human somatostatin receptor, hSSTR2, which is controlled by epigenetic modifications. Endocrinology 149:3137-3147.

Tostivint H, Ocampo Daza D, Bergqvist CA, Quan FB, Bougerol M, Lihrmann I, and Larhammar D (2014) Molecular evolution of GPCRs: somatostatin/urotensin II receptors. J Mol Endocrinol 52:T61-T86.

Toumpanakis C and Caplin ME (2013) Update on the role of somatostatin analogs for the treatment of patients with gastroenteropancreatic neuroendocrine tumors. Semin Oncol 40:56-68.

Tower-Gilchrist C, Lee E, and Sztul E (2011) Endosomal trafficking of the G proteincoupled receptor somatostatin receptor 3. Biochem Biophys Res Commun 413 555-560.

Trejtnar F, Laznicek M, Laznickova A, and Mather S.J (2000) Pharmacokinetics and renal handling of 99mTc-labeled peptides. J Nucl Med 41:177-182.

Treppiedi D, Peverelli E, Giardino E, Ferrante E, Calebiro D, Spada A and Mantovani G (2017) Somatostatin receptor type 2 (SSTR2) internalization and intracellular trafficking in pituitary GH-secreting adenomas: role of scaffold proteins and implications for pharmacological resistance. Horm Metab Res 49 259-268.

Troxler T, Enz A, Hoyer D, Langenegger D, Neumann P, Pfäffli P, Schoeffter P, and Hurth K (2008) Ergoline derivatives as highly potent and selective antagonists at the somatostatin sst 1 receptor. Bioorg Med Chem Lett 18:979-982.

Troxler T, Hurth K, Schuh K-H, Schoeffter P, Langenegger D, Enz A, and Hoyer D (2010) Decahydroisoquinoline derivatives as novel non-peptidic, potent and subtype-selective somatostatin sst(3) receptor antagonists. Bioorg Med Chem Lett 20:1728-1734

Tulipano G and Schulz S (2007) Novel insights in somatostatin receptor physiology. Eur J Endocrinol 156 (Suppl 1):S3-S11.

Tulipano G, Stumm R, Pfeiffer M, Kreienkamp H-J, Höllt V, and Schulz S (2004) Differential $\beta$-arrestin trafficking and endosomal sorting of somatostatin receptor subtypes. J Biol Chem 279:21374-21382.

Tuominen I, Heliövaara E, Raitila A, Rautiainen MR, Mehine M, Katainen R, Donner I, Aittomäki V, Lehtonen HJ, Ahlsten M, et al. (2015) AIP inactivation leads to pituitary tumorigenesis through defective Goi-cAMP signaling. Oncogene 34 $1174-1184$

Unger N, Serdiuk I, Sheu SY, Walz MK, Schulz S, Saeger W, Schmid KW, Mann K, and Petersenn S (2008) Immunohistochemical localization of somatostatin receptor subtypes in benign and malignant adrenal tumours. Clin Endocrinol (Oxf) 68:850-857.

Unger N, Ueberberg B, Schulz S, Saeger W, Mann K, and Petersenn S (2012) Differential expression of somatostatin receptor subtype 1-5 proteins in numerous human normal tissues. Exp Clin Endocrinol Diabetes 120:482-489.

Urban-Ciecko J and Barth AL (2016) Somatostatin-expressing neurons in cortical networks. Nat Rev Neurosci 17:401-409.

Valkema R, de Jong M, Bakker WH, Breeman WA, Kooij PP, Lugtenburg PJ, Jong FH, Christiansen A, Kam BL, De Herder WW, et al. (2002) Phase I study of peptide receptor radionuclide therapy with [111In-DTPA0]octreotide: the Rotterdam experience. Semin Nucl Med 32:110-122.

Valsecchi ME, Coronel M, Intenzo CM, Kim SM, Witkiewicz AK, and Sato T (2013) Somatostatin receptor scintigraphy in patients with metastatic uveal melanoma. Melanoma Res 23:33-39.

van der Hoek J, Lamberts SW, and Hofland LJ (2010) The somatostatin receptor subtype 5 in neuroendocrine tumours. Expert Opin Investig Drugs 19:385-399. 
van der Pas R, Feelders RA, Gatto F, de Bruin C, Pereira AM, van Koetsveld PM, Sprij-Mooij DM, Waaijers AM, Dogan F, Schulz S, et al. (2013) Preoperative normalization of cortisol levels in Cushing's disease after medical treatment: consequences for somatostatin and dopamine receptor subtype expression and in vitro response to somatostatin analogs and dopamine agonists. J Clin Endocrinol Metab 98:E1880-E1890.

van Eersel MEA, Meeuwisse-Pasterkamp SH, Muller Kobold AC, Meiners LC, den Dunnen WF, Hofland LJ, and van den Berg G (2017) Treatment of a thyrotropinsecreting pituitary adenoma (TSH-oma) with pasireotide LAR. Clin Endocrinol (Oxf) 87:877-879.

van Essen M, Krenning EP, Kam BL, de Herder WW, van Aken MO, and Kwekkeboom DJ (2008) Report on short-term side effects of treatments with $177 \mathrm{Lu}$-octreotate in combination with capecitabine in seven patients with gastroenteropancreatic neuroendocrine tumours. Eur J Nucl Med Mol Imaging $\mathbf{3 5}$ $743-748$

Vanetti M, Kouba M, Wang X, Vogt G, and Höllt V (1992) Cloning and expression of a novel mouse somatostatin receptor (SSTR2B). FEBS Lett 311:290-294.

Vanhoutte PM, Humphrey PP, and Spedding M (1996) X. International Union of Pharmacology recommendations for nomenclature of new receptor subtypes. Pharmacol Rev 48:1-2.

Van Op den Bosch J, Torfs P, De Winter BY, De Man JG, Pelckmans PA, Van Marck E, Grundy D, Van Nassauw L, and Timmermans JP (2009) Effect of genetic SSTR4 ablation on inflammatory peptide and receptor expression in the non-inflamed and inflamed murine intestine. J Cell Mol Med 13:3283-3295.

Van Op den Bosch J, van Nassauw L, Lantermann K, van Marck E and Timmermans JP (2007) Effect of intestinal inflammation on the cell-specific expression of somatostatin receptor subtypes in the murine ileum. Neurogastroenterol Motil 19:596-606.

Vasilaki A, Papasava D, Hoyer D, and Thermos K (2004) The somatostatin receptor (sst1) modulates the release of somatostatin in the nucleus accumbens of the rat. Neuropharmacology 47:612-618.

Vasilaki A and Thermos K (2009) Somatostatin analogues as therapeutics in retinal disease. Pharmacol Ther 122:324-333.

Vaudry H, Leprince J, Chatenet D, Fournier A, Lambert DG, Le Mével J-C, Ohlstein EH, Schwertani A, Tostivint H, and Vaudry D (2015) International Union of Basic and Clinical Pharmacology. XCII. Urotensin II, urotensin II-related peptide, and their receptor: from structure to function. Pharmacol Rev 67:214-258.

Veenstra MJ, de Herder WW, Feelders RA, and Hofland LJ (2013) Targeting the somatostatin receptor in pituitary and neuroendocrine tumors. Expert Opin Ther Targets 17:1329-1343.

Verhoef C, van Dekken H, Hofland LJ, Zondervan PE, de Wilt JH, van Marion R, de Man RA, IJzermans JN, and van Eijck CH (2008) Somatostatin receptor in human hepatocellular carcinomas: biological, patient and tumor characteristics. Dig Surg 25:21-26.

Vernejoul F, Faure P, Benali N, Calise D, Tiraby G, Pradayrol L, Susini C, and Buscail L (2002) Antitumor effect of in vivo somatostatin receptor subtype 2 gene transfer in primary and metastatic pancreatic cancer models. Cancer Res 62:6124-6131.

Vezzani A and Hoyer D (1999) Brain somatostatin: a candidate inhibitory role in seizures and epileptogenesis. Eur J Neurosci 11:3767-3776.

Videau C, Hochgeschwender U, Kreienkamp HJ, Brennan MB, Viollet C, Richter D, and Epelbaum J (2003) Characterisation of [125I]-Tyr0DTrp8-somatostatin binding in sst1- to sst4- and SRIF-gene-invalidated mouse brain. Naunyn Schmiedebergs Arch Pharmacol 367:562-571.

Viollet C, Lepousez G, Loudes C, Videau C, Simon A, and Epelbaum J (2008) Somatostatinergic systems in brain: networks and functions. Mol Cell Endocrinol 286:75-87.

Viollet C, Vaillend C, Videau C, Bluet-Pajot MT, Ungerer A, L’Héritier A, Kopp C, Potier B, Billard J, Schaeffer J, et al. (2000) Involvement of sst2 somatostatin receptor in locomotor, exploratory activity and emotional reactivity in mice. Eur $J$ Neurosci 12:3761-3770.

Vitali E, Cambiaghi V, Zerbi A, Carnaghi C, Colombo P, Peverelli E, Spada A Mantovani G, and Lania AG (2016) Filamin-A is required to mediate SST2 effects in pancreatic neuroendocrine tumours. Endocr Relat Cancer 23:181-190.

Vlotides G, Cruz-Soto M, Rubinek T, Eigler T, Auernhammer CJ, and Melmed S (2006) Mechanisms for growth factor-induced pituitary tumor transforming gene-1 expression in pituitary folliculostellate TtT/GF cells. Mol Endocrinol 20: $3321-3335$

Vockel M, Breitenbach U, Kreienkamp H-J, and Brandner JM (2010) Somatostatin regulates tight junction function and composition in human keratinocytes. Exp Dermatol 19:888-894.

Vockel M, Pollok S, Breitenbach U, Ridderbusch I, Kreienkamp H-J, and Brandner JM (2011) Somatostatin inhibits cell migration and reduces cell counts of human keratinocytes and delays epidermal wound healing in an ex vivo wound model. PLoS One 6:e19740.

Wagner HN Jr, Burns HD, Dannals RF, Wong DF, Langstrom B, Duelfer T, Frost JJ, Ravert HT, Links JM, Rosenbloom SB, et al. (1983) Imaging dopamine receptors in the human brain by positron tomography. Science 221:1264-1266.

Waldherr C, Pless M, Maecke HR, Haldemann A, and Mueller-Brand J (2001) The clinical value of [90Y-DOTA]-D-Phe1-Tyr3-octreotide (90Y-DOTATOC) in the treatment of neuroendocrine tumours: a clinical phase II study. Ann Oncol 12 941-945.

Wang XP, Norman MA, Yang J, Cheung A, Moldovan S, Demayo FJ, and Brunicardi FC (2004) Double-gene ablation of SSTR1 and SSTR5 results in hyperinsulinemia and improved glucose tolerance in mice. Surgery 136:585-592.

Wang XP, Yang J, Norman MA, Magnusson J, DeMayo FJ, and Brunicardi FC (2005) SSTR5 ablation in islet results in alterations in glucose homeostasis in mice. FEBS Lett 579:3107-3114.

Wang Y, Wang W, Jin K, Fang C, Lin Y, Xue L, Feng S, Zhou Z, Shao C, Chen M, et al. (2017) Somatostatin receptor expression indicates improved prognosis in gastroenteropancreatic neuroendocrine neoplasm, and octreotide long-acting release is effective and safe in Chinese patients with advanced gastroenteropancreatic neuroendocrine tumors. Oncol Lett 13:1165-1174.

War SA, Kim B, and Kumar U (2015) Human somatostatin receptor-3 distinctively induces apoptosis in MCF-7 and cell cycle arrest in MDA-MB-231 breast cancer cells. Mol Cell Endocrinol 413:129-144.

Waser B, Cescato R, Liu Q, Kao YJ, Körner M, Christ E, Schonbrunn A, and Reubi JC (2012) Phosphorylation of sst2 receptors in neuroendocrine tumors after octreotide treatment of patients. Am J Pathol 180:1942-1949.

Weckbecker G, Briner U, Lewis I, and Bruns C (2002) SOM230: a new somatostatin peptidomimetic with potent inhibitory effects on the growth hormone/insulin-like growth factor-I axis in rats, primates, and dogs. Endocrinology 143:4123-4130.

Wente W, Efanov AM, Treinies I, Zitzer H, Gromada J, Richter D, and Kreienkamp HJ (2005a) The PDZ/coiled-coil domain containing protein PIST modulates insulin secretion in MIN6 insulinoma cells by interacting with somatostatin receptor subtype 5. FEBS Lett 579:6305-6310.

Wente W, Stroh T, Beaudet A, Richter D, and Kreienkamp HJ (2005b) Interactions with PDZ domain proteins PIST/GOPC and PDZK1 regulate intracellular sorting of the somatostatin receptor subtype 5. J Biol Chem 280:32419-32425.

Wester H-J, Schottelius M, Poethko T, Bruus-Jensen K, and Schwaiger M (2004) Radiolabeled carbohydrated somatostatin analogs: a review of the current status. Cancer Biother Radiopharm 19:231-244.

Wester HJ, Schottelius M, Scheidhauer K, Meisetschläger G, Herz M, Rau FC, Reubi JC, and Schwaiger M (2003) PET imaging of somatostatin receptors: design, synthesis and preclinical evaluation of a novel $18 \mathrm{~F}$-labelled, carbohydrated analogue of octreotide. Eur $J$ Nucl Med Mol Imaging 30:117-122.

White RE, Schonbrunn A, and Armstrong DL (1991) Somatostatin stimulates $\mathrm{Ca}(2$ +)-activated $\mathrm{K}+$ channels through protein dephosphorylation. Nature 351:570-573. Wild D, Fani M, Behe M, Brink I, Rivier JEF, Reubi JC, Maecke HR, and Weber WA (2011) First clinical evidence that imaging with somatostatin receptor antagonists is feasible. $J$ Nucl Med 52:1412-1417.

Wild D, Mäcke HR, Waser B, Reubi JC, Ginj M, Rasch H, Müller-Brand J, and Hofmann M (2005) 68Ga-DOTANOC: a first compound for PET imaging with high affinity for somatostatin receptor subtypes 2 and 5. Eur J Nucl Med Mol Imaging 32:724.

Wild D, Schmitt JS, Ginj M, Mäcke HR, Bernard BF, Krenning E, De Jong M, Wenger S, and Reubi J-C (2003) DOTA-NOC, a high-affinity ligand of somatostatin receptor subtypes 2, 3 and 5 for labelling with various radiometals. Eur J Nucl Med Mol Imaging 30:1338-1347.

Wilkinson GF, Feniuk W, and Humphrey PP (1997a) Characterization of human recombinant somatostatin sst5 receptors mediating activation of phosphoinositide metabolism. Br J Pharmacol 121:91-96.

Wilkinson GF, Feniuk W, and Humphrey PP (1997b) Homologous and heterologous desensitisation of somatostatin-induced increases in intracellular $\mathrm{Ca} 2+$ and inositol 1,4,5-trisphosphate in CHO-K1 cells expressing human recombinant somatostatin sst5 receptors. Eur J Pharmacol 340:277-285.

Williams G, Ball JA, Lawson RA, Joplin GF, Bloom SR, and Maskill MR (1987) Analgesic effect of somatostatin analogue (octreotide) in headache associated with pituitary tumours. Br Med J (Clin Res Ed) 295:247-248.

Woelfl S, Bogner S, Huber H, Salaheddin-Nassr S, Hatzl M, Decristoforo C, Virgolini I, and Gabriel M (2014) Expression of somatostatin receptor subtype 2 and subtype 5 in thyroid malignancies. Nucl Med (Stuttg) 53:179-185.

Wright JW and Harding JW (2011) Brain renin-angiotensin-a new look at an old system. Prog Neurobiol 95:49-67.

Xu Y, Berelowitz M, and Bruno JF (1995a) Dexamethasone regulates somatostatin receptor subtype messenger ribonucleic acid expression in rat pituitary $\mathrm{GH} 4 \mathrm{C} 1$ cells. Endocrinology 136:5070-5075.

$\mathrm{Xu}$ Y, Bruno JF, and Berelowitz M (1995b) Characterization of the proximal promoter region of the rat somatostatin receptor gene, SSTR4. Biochem Biophys Res Commun 206:935-941.

Xu Y, Song J, Berelowitz M, and Bruno JF (1993) Ligand binding and functional properties of the rat somatostatin receptor SSTR4 stably expressed in Chinese hamster ovary cells. Mol Cell Neurosci 4:245-249.

Yamada Y, Kagimoto S, Kubota A, Yasuda K, Masuda K, Someya Y, Ihara Y, Li Q Imura H, Seino S, et al. (1993) Cloning, functional expression and pharmacological characterization of a fourth (hSSTR4) and a fifth (hSSTR5) human somatostatin receptor subtype. Biochem Biophys Res Commun 195:844-852.

Yamada Y, Post SR, Wang K, Tager HS, Bell GI, and Seino S (1992a) Cloning and functional characterization of a family of human and mouse somatostatin receptors expressed in brain, gastrointestinal tract, and kidney. Proc Natl Acad Sci USA 89 251-255

Yamada Y, Reisine T, Law SF, Ihara Y, Kubota A, Kagimoto S, Seino M, Seino Y, Bell GI, and Seino S (1992b) Somatostatin receptors, an expanding gene family: cloning and functional characterization of human SSTR3, a protein coupled to adenyly cyclase. Mol Endocrinol 6:2136-2142.

Yamamoto J, Ohnuma K, Hatano R, Okamoto T, Komiya E, Yamazaki H, Iwata S, Dang NH, Aoe K, Kishimoto T, et al. (2014) Regulation of somatostatin receptor 4-mediated cytostatic effects by CD26 in malignant pleural mesothelioma. $\mathrm{Br} J$ Cancer 110:2232-2245.

Yang L, Guo L, Pasternak A, Mosley R, Rohrer S, Birzin E, Foor F, Cheng K, Schaeffer J, and Patchett AA (1998) Spiro[1H-indene-1,4'-piperidine] derivatives as potent and selective non-peptide human somatostatin receptor subtype 2 (sst2) agonists. J Med Chem 41:2175-2179.

Yao JC, Hassan M, Phan A, Dagohoy C, Leary C, Mares JE, Abdalla EK, Fleming JB, Vauthey J-N, Rashid A, et al. (2008) One hundred years after "carcinoid": epidemiology of and prognostic factors for neuroendocrine tumors in 35,825 cases in the United States. J Clin Oncol 26:3063-3072.

Yasuda K, Rens-Domiano S, Breder CD, Law SF, Saper CB, Reisine T, and Bell GI (1992) Cloning of a novel somatostatin receptor, SSTR3, coupled to adenylylcyclase. J Biol Chem 267:20422-20428.

Yin H, Lee KE, Park SA, Bhattarai JP, Suh BJ, Jeon JG, Kim BG, Park SJ, and Han SK (2009) Inhibitory effects of somatostatin on the substantia gelatinosa neurons 
of trigeminal subnucleus caudalis via somatostatin type 2 receptors in juvenile mice. Brain Res 1304:49-56.

Yosten GLC, Elrick MM, Salvatori A, Stein LM, Kolar GR, Ren J, Corbett JA and Samson WK (2015) Understanding peptide biology: the discovery and characterization of the novel hormone, neuronostatin. Peptides 72:192-195.

Zambre Y, Ling Z, Chen M-C, Hou X, Woon C-W, Culler M, Taylor JE, Coy DH, Van Schravendijk C, Schuit F, et al. (1999) Inhibition of human pancreatic islet insulin release by receptor-selective somatostatin analogs directed to somatostatin receptor subtype 5. Biochem Pharmacol 57:1159-1164.

Zamora V, Cabanne A, Salanova R, Bestani C, Domenichini E, Marmissolle F, Giacomi N, O'Connor J, Mendez G, and Roca E (2010) Immunohistochemical expression of somatostatin receptors in digestive endocrine tumours. Dig Liver Dis 42:220-225.

Zatelli MC, Tagliati F, Piccin D, Taylor JE, Culler MD, Bondanelli M, and degli Uberti EC (2002) Somatostatin receptor subtype 1-selective activation reduces cell growth and calcitonin secretion in a human medullary thyroid carcinoma cell line. Biochem Biophys Res Commun 297:828-834.

Zatelli MC, Tagliati F, Taylor JE, Rossi R, Culler MD, and degli Uberti EC (2001) Somatostatin receptor subtypes 2 and 5 differentially affect proliferation in vitro of the human medullary thyroid carcinoma cell line tt. J Clin Endocrinol Metab 86:2161-2169.

Zeggari M, Esteve JP, Rauly I, Cambillau C, Mazarguil H, Dufresne M, Pradayrol L, Chayvialle JA, Vaysse N, and Susini C (1994) Co-purification of a protein tyrosine phosphatase with activated somatostatin receptors from rat pancreatic acinar membranes. Biochem J 303:441-448.

Zeng M, Shang Y, Guo T, He Q, Yung WH, Liu K, and Zhang M (2016) A binding site outside the canonical PDZ domain determines the specific interaction between Shank and SAPAP and their function. Proc Natl Acad Sci USA 113:E3081-E3090.
Zhang C, Yan C, Ren M, Li A, Quan T, Gong H, and Yuan J (2017) A platform for stereological quantitative analysis of the brain-wide distribution of type-specific neurons. Sci Rep 7:14334.

Zhang WH, Beaudet A, and Tannenbaum GS (1999) Sexually dimorphic expression of sst1 and sst2 somatostatin receptor subtypes in the arcuate nucleus and anterior pituitary of adult rats. $J$ Neuroendocrinol 11:129-136.

Zhao P, Canals M, Murphy JE, Klingler D, Eriksson EM, Pelayo JC, Hardt M, Bunnett NW, and Poole DP (2013) Agonist-biased trafficking of somatostatin receptor 2A in enteric neurons. J Biol Chem 288:25689-25700.

Zhao W-Y, Zhuang C, Xu J, Wang M, Zhang Z-Z, Tu L, Wang C-J, Ling T-L, Cao H, and Zhang Z-G (2014) Somatostatin receptors in gastrointestinal stromal tumors: new prognostic biomarker and potential therapeutic strategy. Am J Transl Res 6 : 831-840.

Zheng H, Bailey A, Jiang M-H, Honda K, Chen HY, Trumbauer ME, Van der Ploeg LHT, Schaeffer JM, Leng G, and Smith RG (1997) Somatostatin receptor subtype 2 knockout mice are refractory to growth hormone-negative feedback on arcuate neurons. Mol Endocrinol 11:1709-1717.

Zhou G, Gingras MC, Liu SH, Li D, Li Z, Catania RL, Stehling KM, Li M, Paganelli G, Gibbs RA, et al. (2011) The hypofunctional effect of P335L single nucleotide polymorphism on SSTR5 function. World J Surg 35:1715-1724.

Zhou G, Liu SH, Shahi KM, Wang H, Duan X, Lin X, Feng XH, Li M, Fisher WE Demayo FJ, et al. (2012) Negative regulation of pancreatic and duodenal homeobox-1 by somatostatin receptor subtype 5. Mol Endocrinol 26:1225-1234.

Zitzer H, Richter D, and Kreienkamp H-J (1999) Agonist-dependent interaction of the rat somatostatin receptor subtype 2 with cortactin-binding protein 1 . J Biol Chem 274:18153-18156. 